\title{
(Epi-) genetic profiling of colorectal cancer : prognostic and biological relevance, with emphasis on tumor-hypoxia
}

Citation for published version (APA):

Cleven, A. H. G. (2012). (Epi-) genetic profiling of colorectal cancer : prognostic and biological relevance, with emphasis on tumor-hypoxia. [Doctoral Thesis, Maastricht University]. Maastricht University. https://doi.org/10.26481/dis.20120601ac

Document status and date:

Published: 01/01/2012

DOI:

10.26481/dis.20120601ac

Document Version:

Publisher's PDF, also known as Version of record

Please check the document version of this publication:

- A submitted manuscript is the version of the article upon submission and before peer-review. There can be important differences between the submitted version and the official published version of record.

People interested in the research are advised to contact the author for the final version of the publication, or visit the DOI to the publisher's website.

- The final author version and the galley proof are versions of the publication after peer review.

- The final published version features the final layout of the paper including the volume, issue and page numbers.

Link to publication

\footnotetext{
General rights rights.

- You may freely distribute the URL identifying the publication in the public portal. please follow below link for the End User Agreement:

www.umlib.nl/taverne-license

Take down policy

If you believe that this document breaches copyright please contact us at:

repository@maastrichtuniversity.nl

providing details and we will investigate your claim.
}

Copyright and moral rights for the publications made accessible in the public portal are retained by the authors and/or other copyright owners and it is a condition of accessing publications that users recognise and abide by the legal requirements associated with these

- Users may download and print one copy of any publication from the public portal for the purpose of private study or research.

- You may not further distribute the material or use it for any profit-making activity or commercial gain

If the publication is distributed under the terms of Article 25fa of the Dutch Copyright Act, indicated by the "Taverne" license above, 


\section{(Epi-) Genetic Profiling of Colorectal Cancer:}

Prognostic and Biological Relevance, with emphasis on tumor-hypoxia 
(C) Arjen H.G. Cleven, Maastricht 2012

Layout: Tiny Wouters

Cover design: Annette Jost

Production: Wöhrmann Print Service

ISBN: 978-94-6203-030-5 


\section{(Epi-) Genetic Profiling of Colorectal Cancer:}

\section{Prognostic and Biological Relevance, with emphasis on tumor-hypoxia}

\section{PROEFSCHRIFT}

Ter verkrijging van de graad van doctor aan de Universiteit Maastricht, op gezag van de Rector Magnificus,

Prof. Mr. G.P.M.F. Mols,

volgens het besluit van het College van Decanen,

in het openbaar te verdedigen

op vrijdag 1 juni 2012 om 14.00 uur

door

Arjen H.G. Cleven 


\section{Promotores}

Prof. dr. A.P. de Bruïne

Prof. dr. M. van Engeland

Copromotor

Dr. S. Derks

\section{Beoordelingscommissie}

Prof. dr. F.C.S. Ramaekers (voorzitter)

Dr. G. Beets

Prof. dr. A. zur Hausen

Prof. dr. A.A.M. Masclee

Prof. dr. G.A. Meijer, VUMC Amsterdam 
"Ik weet dat je gaat promoveren, maar weet ook, dat als je niet promoveert, ik je een fantastische zoon vind."

Ger Cleven, ${ }^{\dagger} 12$ maart 2010 



\section{Contents}

$\begin{array}{ll}\text { Abbreviations } & 9\end{array}$

$\begin{array}{lll}\text { Chapter } 1 \quad \text { General Introduction } & 11\end{array}$

Chapter 2 Pharmacoepigenomics in colorectal cancer; a step 19 forward in predicting prognosis and treatment response

Chapter 3 Computer assisted (epi-)genotypic categorization of colorectal cancer identifies CIMP and TP53 mutations as leading classification tools

Chapter $4 \quad$ CHFR promoter CpG island methylation is an indicator of poor prognosis in stage II microsatellite stable colorectal cancer

Chapter 5 Emerging evidence for $C H F R$ as a cancer biomarker: update and prospects

Chapter 6 Stromal expression of hypoxia regulated proteins is an adverse prognostic factor in colorectal carcinomas

Chapter 7 Promoter $\mathrm{CpG}$ island hypermethylation of cancer associated genes is associated with stromal expression of hypoxia markers in colorectal cancer

Chapter 8 Poorer outcome in stromal HIF-2 $\alpha$ and CA9 positive colorectal adenocarcinomas is associated with Wild-type TP53 but not with BNIP3 promoter hypermethylation or apoptosis

Chapter 9 General discussion

Summary

Samenvatting

Dankwoord

Curriculum vitae

List of publications 



\section{List of abbreviations}

\begin{tabular}{|c|c|}
\hline AHEAD & Alliance for the Human Epigenome and Disease \\
\hline AJCC & American Joint Committee on Cancer \\
\hline ASCO & American Society of Clinical Oncology \\
\hline CA9 & carbonic anhydrase 9 \\
\hline CAN & cancer candidate genes \\
\hline ChIP & chromatin immunoprecipitation \\
\hline CIMP & CpG island methylator phenotype \\
\hline CIN & chromosomal instability \\
\hline COBRA & combined bisulfite restriction analysis \\
\hline CRC & colorectal cancer \\
\hline DAC & 5-aza-2'-deoxycytidine \\
\hline DNA & deoxyribonucleic acid \\
\hline DNMT & DNA methyltransferase \\
\hline EPC's & endothelial progenitor cells \\
\hline 5-FU & 5-fluorouracil \\
\hline FAP & Familial Adenomatous Polyposis \\
\hline GLUT1 & glucose transporter 1 \\
\hline HDAC & histone deacetylases \\
\hline HIF & hypoxia-inducible factor \\
\hline HNPCC & Hereditary Non-Polyposis Colon Cancer \\
\hline $\mathrm{HR}$ & hazard ratio \\
\hline HRE & hypoxia responsive element \\
\hline $\mathrm{IHC}$ & immunohistochemistry \\
\hline $\mathrm{LOH}$ & loss of heterozygosity \\
\hline MBD & methyl binding domain \\
\hline MCA & methylated $\mathrm{CpG}$ island amplification \\
\hline MeDIP & methylated DNA immunoprecipitation \\
\hline MI & methylation index \\
\hline MIN & microsatellite instable \\
\hline MIRA & methylated-CpG island recovery assay \\
\hline MLPA & multiplex ligation probe amplification \\
\hline MMR & mismatch repair \\
\hline MSI & microsatellite instability \\
\hline MSP & methylation specific PCR \\
\hline MSS & microsatellite stability \\
\hline NSCLC & non-small cell lung cancer \\
\hline PCR & polymerase chain reaction \\
\hline sDNA & stool DNA test \\
\hline SNP & single nucleotide polymorphism \\
\hline
\end{tabular}


TNM tumor-node-metastasis

TS thymidylate synthase

TSA Trichostatin A

UICC International Union against Cancer, together with the American Joint Committee on Cancer

WHO World Health Organization 


\section{Chapter 1}

General introduction 
$12 \mid$ Chapter 1 
General introduction $\mid 13$

\section{General introduction}

Colorectal cancer (CRC) is one of the most common cancer types worldwide ${ }^{1}$. In Europe 436.000 new CRC cases were diagnosed and 212.000 patients died of this disease in $2008^{2}$. CRC occurs sporadically $(70 \%)$, in families $(25 \%)$ and as the inherited colon cancer syndromes Lynch syndrome/Hereditary NonPolyposis Colon Cancer (HNPCC), Familial Adenomatous Polyposis (FAP) and MUTYH-associated polyposis in approximately $5 \%$ of cases $^{3}$.

CRC can be treated and often cured by surgery when localized to the submucosa or muscularis propria of the bowel (stage I) ${ }^{1}$. Importantly, stage II $\mathrm{CRC}$ patients (cancer progressed into subserosa or perforating the visceral peritoneum) undergo surgery alone, despite the recognition that a subgroup with a poor prognosis would probably benefit from adjuvant chemotherapy. Within stage III (lymph node metastases) and stage IV (distant organ metastases) CRC, adjuvant chemotherapeutic drugs such as fluoropyrimidines, irinotecan and oxaliplatin are now used as part of standard care and have been shown to improve survival significantly ${ }^{4}$. Although the anatomical based staging system predicts the survival accurately, variation in the course of the disease and response to treatment among individuals within the same stage exists. Emerging understanding of the underlying biology of CRC is expected to identify tumor specific molecular markers that improve risk assessment and therapeutic options within different stages of $C R C^{5}$.

The last decades, comprehensive analysis of the CRC (epi)genome has provided novel information on the biology underlying CRC carcinogenesis. These studies revealed that cancer cells have acquired genomic instability, enabling limitless replicative potential, tissue invasion and metastasis, sustained angiogenesis, evasion of apoptosis, self-sufficiency in growth signals and insensitivity to anti-growth signaling ${ }^{6-8}$. The underlying cause of these socalled hallmarks of cancer are genetic and epigenetic alterations which can be subdivided in CRC into three categories: chromosomal instability (CIN), microsatellite instability (MSI) and $\mathrm{CpG}$ island methylator phenotype (CIMP) ${ }^{9-13}$. $\mathrm{CIN}$ is characterised by numerical and/or structural chromosomal abnormalities, increased rate of loss of heterozygosity ( $\mathrm{LOH})$ and mutations in the tumor suppressor genes adenomatous polyposis coli (APC), v-Ki-ras2 Kirsten rat sarcoma viral oncogene homolog (KRAS) and tumor protein p53 $(T P 53)^{12-14}$. MSI results from inactivation of the mismatch repair (MMR) system, caused by germline mutations in MMR genes mutS homolog 2, colon cancer, nonpolyposis type 1 (E. coli) (MSH2), mutS homolog 6 (E. coli) (MSH6) and PMS2 postmeiotic segregation increased 2 (S. cerevisiae) (PMS2) in Lynch syndrome/HNPCC ${ }^{15}$ and promoter $\mathrm{CpG}$ island methylation of mutL homolog1, colon cancer, nonpolyposis type 2 (E.Coli) (MLH1) in sporadic colorectal cancer $^{16}$. CIMP CRCs are characterized by frequent promoter $\mathrm{CpG}$ island 
methylation and subsequent silencing of tumor suppressor genes and DNA repair genes and are associated with $\mathrm{MSI}$ and $v$-raf murine sarcoma viral oncogene homolog B1 (BRAF) mutations ${ }^{17-19}$. CRCs are hypothesized to develop through one of the three routes: CIN, MSI or CIMP, eventually resulting in the inactivation of tumor suppressor genes and activation of oncogenes and thereby achieving the hallmarks of cancer ${ }^{16,20}$. This illustrates that $\mathrm{CRC}$ is a heterogeneous disease consisting of subgroups with a different biology and each subgroup of CRC is associated with more or less specific morphological features. For example MSI, BRAF mutated and CIMP CRCs are associated with a serrated and/or mucinous morphology ${ }^{21,22}$, whereas the presence of dirty necrosis in malignant glandular lumina is negatively correlated with $\mathrm{MSI}$ and thought to be primarily present in CRCs with a high frequency of $A P C$ en TP53 mutations (CIN pathway) ${ }^{23}$.

Increased proliferation, which is one of the hallmarks of cancer cells, especially at a stage that the tumor has grown beyond a size that can be fed by the surrounding micro-vessels, induces hypoxia ${ }^{8,24}$. Hypoxia is defined as an oxygen tension below the physiological level, and occurs frequently in solid tumors $^{25}$. In CRC, the importance of hypoxia has been demonstrated by clinical studies in which hypoxia predicts for worse outcome and resistance to chemotherapy and radiotherapy ${ }^{26,27}$. Understanding the underlying biology explaining how CRCs will adapt to a hypoxic environment will improve patient risk assessment and predict response to chemotherapy ${ }^{28}$. The coordinated cellular response to hypoxia that influences the pattern of gene expression is mediated by hypoxia-inducible factors (HIFs) and other cellular pathways such as translation regulation, microRNA induction and chromatin remodelling ${ }^{29,30}$. Furthermore, under chronic hypoxic conditions epigenetic changes occur, notably a genome-wide adjustment of promoter $\mathrm{CpG}$ island methylation, suggesting an adaptation to the altered environment thereby promoting and maintaining a hypoxia-adapted cellular phenotype, with a potential role in tumor development ${ }^{31}$.

Regarding HIF's, there are three homologues of the alpha subunit (HIF-1 $\alpha$, HIF-2a, HIF-3a $)^{32}$. HIF-1 $\alpha$ is the best understood isoform and together with HIF-2 $\alpha$, both transcription factors are thought to play a significant role in tumor neovascularization, enhanced cellular proliferation, decreased apoptosis, and development of resistance to chemotherapeutic agents ${ }^{30,33-35}$. Studying the physiology of tumor hypoxia also implies studying the downstream effects of HIF. These downstream effects are regulated amongst others by vascular endothelial growth factor (VEGF) involved in angiogenesis, glucose transporter 1 (GLUT1) involved in glycolysis, carbonic anhydrase IX (CA9) regulation of $\mathrm{pH}$, TP53 and BCL2/adenovirus E1B 19kDa interacting protein 3 (BNIP3) involved in regulation of apoptosis and autophagy ${ }^{36,37}$. 
General introduction $\mid 15$

In understanding the mechanisms through which tumor hypoxia can induce a more aggressive phenotype, attention should be directed to both the epithelial and stromal tumor compartments. This is illustrated by a recent study in breast cancer showing that not only the epithelial compartment of the tumor, but also the stromal compartment, consisting of (myo)-fibroblasts, endothelial cells, smooth muscle cells, adipocytes, inflammatory cells and nerve cells, has undergone changes in gene expression. The most prominently altered genes included hypoxia-associated genes and this gene expression profile was associated with clinical outcome ${ }^{38}$. Hypoxia-driven cell biological and metabolic changes might alter the tumor stroma towards an environment that can facilitate cancer progression ${ }^{39}$. Cancer development depends upon changes in the interactions between epithelial cells and their surrounding stroma, leading to biological effects along several important pathways so-called "hallmarks of cancer", that collectively constitute malignant growth ${ }^{8}$. Studying these biological processes and correlate it with patient outcome, will besides a better understanding of the underlying tumor biology, identify molecular markers that could improve therapeutic options within CRC, additional to the traditional staging method.

\section{Aim and outline of thesis}

The identification of prognostic markers for $\mathrm{CRC}$, to determine risk profile and to identify patients that need adjuvant therapy respectively, is urgently needed. The practice of medicine could benefit from integration of genomic and epigenomic information from both the tumor and stroma, to accurately determine prognosis and select the best therapies for the longest durable responses and the lowest likehood of toxicity.

The aim of this thesis was to study genetic and epigenetic alterations in relation to morphological features and hypoxia in CRC, providing insight in the underlying biology of this cancer and possibly yielding relevant novel prognostic information. Chapter 2 reviews the current knowledge on the epidemiology, diagnosis, treatment and the prognostic and predictive value of (epi)genetic markers in CRC. In chapter 3 we classified CRC by computer assisted unsupervised hierarchical clustering, based on key genetic and epigenetic events in CRC including: CIMP, MSI, and APC-, KRAS-, TP53-, and $B R A F$ mutation status, in a series of 160 CRC cases. Subsequently, we investigated whether these molecular clusters are associated with morphological features, patient characteristics and prognosis. In addition, we evaluated the prognostic significance of promoter $\mathrm{CpG}$ island methylation of a variety of tumor suppressor- and DNA repair genes in CRC patients treated with surgery alone, taking into account MSI, BRAF and KRAS mutations (chapter 4). Chapter 5 summarizes the current evidence of altered CHFR 
expression in cancer and discusses its promising role as a prognostic and predictive biomarker.

Promoter $\mathrm{CpG}$ island methylation and associated gene expression alterations, has been reported to play a role in hypoxia. In chapter 6 we studied the baseline protein expression of four hypoxia induced markers (hypoxia-inducible

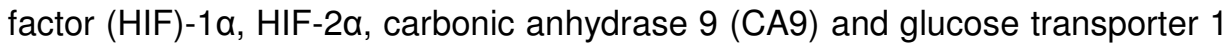
(GLUT1)), in both the epithelial and stromal compartment of CRC's, and evaluated their association with clinicopathological parameters. In chapter $\mathbf{7}$, we investigated whether hypoxia in CRC is related to promoter $\mathrm{CpG}$ island methylation of a series of genes frequently and functionally methylated hallmark of cancer genes. Understanding the mechanisms by which hypoxic tumors can overcome cell death signals (one of the hallmarks of cancer) and adapt is critical for our understanding of tumor progression and development of effective therapeutics in CRC patients with adverse prognostic profiles. Therefore in chapter 8, we attempt to elucidate whether changes in the epithelial cell compartment of CRC, such as apoptosis and concomitant (epi)genetic changes, are related to hypoxia-related changes in the stromal compartment. For this purpose, we correlated alterations of TP53 and BNIP3 in tumor cells with expression of hypoxia-related proteins HIF-2 $\alpha$ and CA9 in relation with patient outcome and apoptotic activity in CRCs. Chapter 9 discusses the major findings and potential clinical applications of our research. 
\begin{tabular}{l|l} 
General introduction & 17
\end{tabular}

\section{References}

1. Ferlay $\mathrm{J}$, Autier $\mathrm{P}$, Boniol $\mathrm{M}$, et al: Estimates of the cancer incidence and mortality in Europe in 2006. Ann Oncol 18:581-92, 2007

2. Ferlay J, Parkin DM, Steliarova-Foucher E: Estimates of cancer incidence and mortality in Europe in 2008. Eur J Cancer, 2010

3. Markowitz SD, Bertagnolli MM: Molecular origins of cancer: Molecular basis of colorectal cancer. N Engl J Med 361:2449-60, 2009

4. Loupakis F, Masi G, Vasile E, et al: First-line chemotherapy in metastatic colorectal cancer: new approaches and therapeutic algorithms. Always hit hard first? Curr Opin Oncol 20:45965, 2008

5. Dotan E, Cohen SJ: Challenges in the management of stage II colon cancer. Semin Oncol 38:511-20, 2011

6. Wood LD, Parsons DW, Jones S, et al: The genomic landscapes of human breast and colorectal cancers. Science 318:1108-13, 2007

7. Yagi K, Akagi K, Hayashi $\mathrm{H}$, et al: Three DNA methylation epigenotypes in human colorectal cancer. Clin Cancer Res 16:21-33, 2010

8. Hanahan D, Weinberg RA: The hallmarks of cancer. Cell 100:57-70, 2000

9. Ogino S, Goel A: Molecular Classification and Correlates in Colorectal Cancer. J Mol Diagn 10:13-27, 2008

10. Shen L, Toyota M, Kondo $\mathrm{Y}$, et al: Integrated genetic and epigenetic analysis identifies three different subclasses of colon cancer. Proc Natl Acad Sci U S A 104:18654-9, 2007

11. Jass JR: Classification of colorectal cancer based on correlation of clinical, morphological and molecular features. Histopathology 50:113-30, 2007

12. Smith G, Carey FA, Beattie J, et al: Mutations in APC, Kirsten-ras, and p53--alternative genetic pathways to colorectal cancer. Proc Natl Acad Sci U S A 99:9433-8, 2002

13. Vogelstein B, Bearon ER, Hamilton SR: Genetic alterations during colorectal-tumor development. New England Journal of Medicine 319:525-32, 1988

14. Hermsen M, Postma C, Baak J, et al: Colorectal adenoma to carcinoma progression follows multiple pathways of chromosomal instability. Gastroenterology 123:1109-19, 2002

15. Lubbe SJ, Webb EL, Chandler IP, et al: Implications of Familial Colorectal Cancer Risk Profiles and Microsatellite Instability Status. J Clin Oncol, 2009

16. Worthley DL, Whitehall VL, Spring KJ, et al: Colorectal carcinogenesis: road maps to cancer. World J Gastroenterol 13:3784-91, 2007

17. Jass JR: Molecular heterogeneity of colorectal cancer: Implications for cancer control. Surg Oncol 16 Suppl 1:S7-9, 2007

18. Wong JJ, Hawkins NJ, Ward RL: Colorectal cancer: a model for epigenetic tumorigenesis. Gut 56:140-8, 2007

19. Weisenberger DJ, Siegmund KD, Campan $M$, et al: $C p G$ island methylator phenotype underlies sporadic microsatellite instability and is tightly associated with BRAF mutation in colorectal cancer. Nat Genet 38:787-93, 2006

20. Taby R, Issa JP: Cancer epigenetics. CA Cancer J Clin 60:376-92, 2010

21. Makinen MJ: Colorectal serrated adenocarcinoma. Histopathology 50:131-50, 2007

22. Tanaka $\mathrm{H}$, Deng $\mathrm{G}$, Matsuzaki $\mathrm{K}$, et al: BRAF mutation, $\mathrm{CpG}$ island methylator phenotype and microsatellite instability occur more frequently and concordantly in mucinous than nonmucinous colorectal cancer. Int J Cancer 118:2765-71, 2006

23. Greenson JK, Bonner JD, Ben-Yzhak O, et al: Phenotype of microsatellite unstable colorectal carcinomas: Well-differentiated and focally mucinous tumors and the absence of dirty necrosis correlate with microsatellite instability. Am J Surg Pathol 27:563-70, 2003

24. Giatromanolaki A, Harris AL: Tumour hypoxia, hypoxia signaling pathways and hypoxia inducible factor expression in human cancer. Anticancer Res 21:4317-24, 2001

25. Pouyssegur J, Dayan F, Mazure NM: Hypoxia signalling in cancer and approaches to enforce tumour regression. Nature 441:437-43, 2006 
26. Koukourakis MI, Giatromanolaki A, Polychronidis A, et al: Endogenous markers of hypoxia/anaerobic metabolism and anemia in primary colorectal cancer. Cancer Sci 97:5828,2006

27. Koukourakis MI, Giatromanolaki A, Sivridis E, et al: Lactate dehydrogenase 5 expression in operable colorectal cancer: strong association with survival and activated vascular endothelial growth factor pathway--a report of the Tumour Angiogenesis Research Group. J Clin Oncol 24:4301-8, 2006

28. Koukourakis MI, Giatromanolaki A, Sivridis E, et al: Prognostic and predictive role of lactate dehydrogenase 5 expression in colorectal cancer patients treated with PTK787/ZK 222584 (vatalanib) antiangiogenic therapy. Clin Cancer Res 17:4892-900, 2011

29. Rankin EB, Giaccia AJ: The role of hypoxia-inducible factors in tumorigenesis. Cell Death Differ 15:678-85, 2008

30. Kenneth NS, Rocha S: Regulation of gene expression by hypoxia. Biochem J 414:19-29, 2008

31. Watson JA, Watson CJ, McCrohan AM, et al: Generation of an epigenetic signature by chronic hypoxia in prostate cells. Hum Mol Genet 18:3594-604, 2009

32. Sivridis E, Giatromanolaki A, Gatter K, et al: Association of Hypoxia-Inducible Factors 1 alpha and 2 alpha with Activated Angiogenic Pathways and Prognosis in Patients with Endometrial Carcinoma. Cancer 95:1055-1063, 2002

33. Shannon AM, Bouchier-Hayes DJ, Condron CM, et al: Tumour hypoxia, chemotherapeutic resistance and hypoxia-related therapies. Cancer Treat Rev 29:297-307, 2003

34. Yoshimura H, Dhar DK, Kohno H, et al: Prognostic Impact of Hypoxia-INducible Factors 1 alpha and 2 alpha in Colorectal Cancer Pateints: Correlation with tumor Angiogenesis and Cyclooxygenase-2 Expression. Clinical Cancer Research 10:8554-8560, 2004

35. Kuwai $T$, Kitadai $Y$, Tanaka $S$, et al: Expression of hypoxia-inducible factor-1 alpha is associated with tumor vascularisation in human colorectal carcinoma. Int. J.Cancer 105:176181,2003

36. Keith B, Simon MC: Hypoxia-inducible factors, stem cells, and cancer. Cell 129:465-72, 2007

37. Bacon AL, Harris AL: Hypoxia-inducible factors and hypoxic cell death in tumour physiology. Ann Med 36:530-9, 2004

38. Finak G, Bertos N, Pepin F, et al: Stromal gene expression predicts clinical outcome in breast cancer. Nat Med 14:518-27, 2008

39. Beppu H, Mwizerwa ON, Beppu Y, et al: Stromal inactivation of BMPRII leads to colorectal epithelial overgrowth and polyp formation. Oncogene 27:1063-70, 2008 


\section{Chapter}

Pharmacoepigenomics in colorectal cancer; a step forward in predicting prognosis and treatment response

KM Smit

AHG Cleven

MP Weijenberg

LAE Hughes

JG Herman

AP de Bruïne

$M$ van Engeland

Pharmacogenomics 2008;9:1903-1916 


\section{Abstract}

Despite therapeutic innovations and increasing education on lifestyle to prevent colorectal cancer (CRC), it is still one of the most common cancer types, and for men the second cause of cancer death. Lately, much attention has been given to identify molecular markers involved in CRC prognosis and treatment with the aim to develop a more accurate classification system based on (epi)genetic alterations and, in addition, find markers that could potentially enhance management of CRC by predicting treatment response in advance. Although many genetic markers have been claimed to have prognostic or predictive influence, results are often inconclusive and, with some exception, they are not used in standard practice. Epigenetic alterations have received less attention although they are probably even more interesting as they can potentially be reversed through drug treatment. This review describes the current knowledge on the prognostic and predictive value of genetic, and especially epigenetic markers in CRC. 
Pharmacoepigenomics in colorectal cancer $\mid 21$

\section{Introduction}

Colorectal cancer (CRC) is one of the most common cancer types worldwide, with annual incidence rates of 401,000 in men and 381,000 in women. Both environmental and (epi)genetic factors play important roles in CRC aetiology. Most CRCs are thought to develop from precursor lesions (adenomas) which can readily be detected and removed by use of endoscopic techniques. The majority of CRCs are sporadic carcinomas, although a small percentage $(<5 \%)$ are due to well-defined inherited syndromes such as familial adenomatous polyposis (FAP) and hereditary non-polyposis colon cancer (HNPCC), currently designated as Lynch syndrome. CRC prognosis and treatment are strongly related to the extent of tumor spread, which makes accurate staging highly important. At this moment, the tumor-node-metastasis (TNM) system is the main tool to provide information on prognosis and to determine treatment protocols ${ }^{1,2}$. However, there is considerable variation in the course of the disease and the treatment response among individuals within the same stage. In recent years, increasing attention has been given to the potential prognostic significance of (epi)genetic markers implicated in CRC, but results of studies are contradictory ${ }^{1}$, mainly due to methodological differences and a lack of standardization $^{2}$. In addition to the impact of molecular markers on prognosis, studies have also focussed on the use of molecular markers to predict or influence treatment outcome. Although some markers seem promising, overall results are inconclusive and further studies are necessary before predictive markers can be used to influence therapeutic decisions in daily practice. A clear distinction should be made between molecular markers that act as independent prognostic markers and markers that can predict treatment outcome. A biomarker can only be considered as an independent prognostic marker if it adds additional information regarding the prognosis to clinical characteristics such as TNM-stage and differentiation grade and does not act as an intermediate factor between clinical prognostic factors and outcome ${ }^{3}$. In addition, to correctly assess whether a biomarker is an independent prognostic factor, it should preferably be studied in an untreated population because treatment is a strong prognostic factor that may bias study results.

\section{Colorectal cancer \& prognosis}

\section{Epidemiology}

CRC is the fourth most common cancer type worldwide. It is estimated that 783,000 new cases are diagnosed each year; a number that has increased rapidly since $1975^{4}$. Fewer than $20 \%$ of cases occur before the age of 50 , and the disease affects men and women almost equally ${ }^{5}$. 
As many as $70 \%$ of CRCs are estimated to develop due to environmental or lifestyle factors. Theoretically, a large number of cases are therefore avoidable. A recent report on diet and cancer concluded that intake of red meat, processed meat, alcohol, body fatness (especially abdominal fat) adult attained height and low levels of physical activity are convincing risk factors for CRC. Intake of calcium and foods containing dietary fibre probably reduces CRC risk. There is only limited evidence for the protective effects of intake of fruits, nonstarchy vegetables, and fish; foods containing folate, selenium and vitamin D; and for the detrimental effects of cheese and foods containing iron, animal fats and sugars ${ }^{6}$.

Large survival differences are observed between CRC patients, even between patients within a similar disease stage. For men it is the second most common cause of cancer death in the European Union ${ }^{5}$. Five year survival of the disease is $90 \%$ if it is diagnosed while still localized, but this drops to only $68 \%$ for regional disease with lymph node involvement and to only $10 \%$ if distant metastases are present ${ }^{7}$.

\section{Clinical diagnosis}

CRC can take many years to develop. Early detection of CRC greatly improves the prognosis, however, first symptoms are usually vague, like weight loss and fatigue. Local (bowel) symptoms including constipation or diarrhea and bloody stools or rectal bleeding are rare and only occur when the tumor has already grown to a considerable size.

Colonoscopy is the golden standard for routine detection of $\mathrm{CRC}^{8}$. It allows for the direct inspection of the colorectal mucosa and biopsy sampling or definitive treatment by polypectomy during the same session ${ }^{9}$. Several CRC screening options are available. These can be subdivided into two categories: (1) structural examinations, which include flexible sigmoidoscopy, colonoscopy, double-contrast barium enema, and computed tomographic colonography and (2) stool tests, which include tests for occult blood or exfoliated DNA.

Molecular knowledge provides a basis for new screening methods that test stool for the presence of DNA alterations that are common in CRC. At present, one commercially stool DNA test (sDNA) is available that includes 21 separate point mutations in genes commonly mutated in CRC and a marker of DNA integrity analysis ${ }^{10}$. However, additional research is necessary to further improve this method ${ }^{11}$. Molecular testing could also be useful to determine CRC risk (e.g. methylation of the SFRP2 gene) ${ }^{12,13}$. 
Pharmacoepigenomics in colorectal cancer $\mid 23$

\section{Pathological diagnosis}

$\mathrm{CRC}$ is classified according to the WHO histological classification system which makes a subdivision into several histological subtypes: mucinous and signet ring cell adenocarcinomas, adenosquamous carcinomas, medullary carcinomas and undifferentiated carcinomas ${ }^{14}$.

The TNM system, as defined by the UICC (International Union against Cancer, together with the American Joint Committee on Cancer), is most commonly used for pathological staging in routine medical practice ${ }^{15}$ and is the most important prognostic factor at the moment. Increasing depth of bowel wall invasion is reflected in the $\mathrm{T}$ stage, a scale of $\mathrm{T} 1$ (invasion of submucosal layer) to T4 (serosal penetration/invasion of adjacent organs). Based on the combination of $\mathrm{T}$ stage, lymph nodes involved $(\mathrm{N})$ and presence of distant metastasis (M), CRCs are grouped in stages from I to IV, with stage I having a $90 \% 5$-year survival, compared to stage IV having a 5-8\% 5-year survival.

\section{Genetics of colorectal cancer}

Apart from traditional histological classification of CRC, increased cancer biology knowledge has led to the idea of classifying CRCs according to (epi)genetic aberrations. CRC is a genetic disease in which accumulation of mutations in oncogenes and tumor suppressor genes plays an important role. In 1990, Fearon and Vogelstein proposed a model for CRC that describes the transition from early adenomas to carcinomas as a consequence of a growing number of somatic mutations in oncogenes and tumor suppressor genes ${ }^{16,17}$. In the adenoma-carcinoma sequence, several well-described genetic aberrations are described, such as APC, KRAS and TP53 ${ }^{18-20}$. Most mutations in APC result in a truncated APC protein ${ }^{21}$ and are considered to occur early in $C R C^{22}$. Loss-of-function mutations in $A P C$ result in nuclear $\beta$-catenin accumulation, a key component of the Wnt signaling transduction pathway ${ }^{23}$, which modulates the expression of downstream Wnt target genes implicated in cell proliferation, migration, differentiation and apoptosis ${ }^{22}$. However, it is highly unlikely that these mutations alone are sufficient for the progression of adenomas ${ }^{24}$. Activating KRAS mutations, found in $40-50 \%$ of CRCs, abolish GTPase activity and as a result, the protein is locked in the active, GTP-bound state. This leads to increased and unregulated cellular proliferation and malignant transformation. TP53 can induce apoptosis or cell cycle arrest to correct occurring defects depending on the severity of the cell damage ${ }^{1}$. Loss-offunction of TP53 is seen in approximately $50 \%$ of CRCs and is considered a late event during the disease ${ }^{17,25}$. Other common genetic defects implicated in colorectal carcinogenesis are BRAF mutations ${ }^{26}$, allelic loss of chromosome $18 \mathrm{q}^{27}$, and alterations in DNA mismatch repair genes (MMR) Approximately $10-15 \%$ of sporadic CRCs show defects in DNA MMR leading to an 
accumulation of alterations in nucleotide repeat sequences; this is defined as microsatellite-instability $(\mathrm{MSI})^{28}$. Most MSI CRCs are believed to arise mainly by epigenetic silencing of the $M L H 1$ repair gene ${ }^{29}$ and are associated with specific tumor characteristics such as proximal location, diploid DNA content, abundant mucin secretion and poor differentiation ${ }^{28}$. Since the proposition of the adenoma-carcinoma sequence, other aberrations frequently seen in CRC have been described and the Fearon and Vogelstein model is constantly being modified to take into account these alternative pathways to $\mathrm{CRC}^{24,30}$. However only a small proportion of genes has been analyzed and the number of genetic alterations that are responsible for the development of CRC is still unknown ${ }^{31}$.

\section{Cancer Candidate Genes (CAN) in colorectal cancer}

The recent determination of the human genome sequence provided the possibility to examine the cancer genome unbiased by systematically screening genetic alterations in cancer instead of focusing on single candidate genes as has been the approach until recently. This has led to the identification of a panel of cancer candidate genes (CAN genes) ${ }^{31}$. CAN genes are most likely to be involved in the neoplastic process and are therefore interesting for further study $^{32}$. Strikingly, mutation frequencies in the CAN genes were relatively low, in the range of $5-15 \%{ }^{31}$. The majority were heterozygous missense mutations (81\%): $59 \%$ of which were $C: G$ to $G: C$ transitions. Moreover, a large part of the mutations (44\%) were found at 5'-CpG-3'dinucleotides ${ }^{31}$. There was also considerable heterogeneity between mutations from different specimens derived from the same tissue. This suggests that single gene alterations reflect only a small part of the tumor mutational spectrum. This could perhaps explain why it has proven to be difficult to find associations between a single gene mutation and prognosis or therapy response ${ }^{31}$. It also confirms the idea that pathways rather than individually mutated genes are responsible for the course of tumorigenesis ${ }^{33}$. Indeed, many mutated CAN genes are involved in phosphatidylinositol 3-kinase (PI3K) signaling or in pathways related to cell adhesion $^{32}$.

\section{Genetic markers and CRC prognosis}

The majority of CRC, about $85 \%$, are characterized by chromosomal instability (CIN) which is associated with microsatellite stability (MSS), aneuploidy and genetic alterations in genes such as KRAS, APC and TP53 ${ }^{34}$. A recent metaanalysis suggested a poorer prognosis for CIN tumors, irrespective of anatomical location or treatment with chemotherapy ${ }^{35}$. Because of this, CIN could be useful for further stratification after standard pathological staging ${ }^{35}$. Nevertheless, individual study results on the prognostic influence of CIN are inconsistent and the 2006 American Society of Clinical Oncology (ASCO) 
Pharmacoepigenomics in colorectal cancer $\mid 25$

guidelines state that measuring $\mathrm{CIN}$ in $\mathrm{CRC}$ is nothing more than an experimental tool ${ }^{35,36}$. Indeed, the question remains whether it is really the effect of CIN that has been measured in previous studies. Individual genetic alterations could possibly act as markers for $\mathrm{CIN}^{35}$. In a number of studies, KRAS has been associated with a poorer prognosis, independent of other prognostic characteristic such as tumor stage in a number of studies. However, other studies were not able to replicate this finding and therefore the clinical relevance of these findings is still unclear $^{1,3,37,38}$. Similarly, inactivation of TP53 and mutations in BRAF have been associated with a poorer prognosis, but not consistently ${ }^{1,3,38-40}$. Previous studies also suggested that loss of chromosome $18 q$ is associated with a poor survival in stage II and stage III patients ${ }^{41,42}$. Gain of chromosome $20 q$ has also been associated with a worse patients survival ${ }^{43}$ In addition, aneuploid or tetraploid DNA content has been associated with a higher tumor stage and distal tumor location ${ }^{44}$, which are known to cause a poor prognosis.

Other genetic markers not associated with CIN have also been suggested to play a role in CRC prognosis. Single Nucleotide Polymorphisms (SNPs) in the gene that codes for thymidylate synthase (TS) (TSER polymorphisms) results in reduced TS expression and have been associated with a reduced CRC risk $^{45}$. Data on the prognostic role of these polymorphisms are inconsistent ${ }^{45-48}$ and independency from tumor stage is only reported in one study ${ }^{46}$. Over expression of TGF- $\beta$ in the primary tumor and in tumor associated-stroma has also been suggested as a prognostic factor in CRC, but results are scarce and for overexpression, independency from tumor stage was not reported ${ }^{3,49-51}$. Similarly, epidermal growth factor receptor (EGFR) expression has been studied in relation to prognosis, but results are inconsistent and an independency of tumor stage has not been proven; a clear relationship between EGFR expression and CRC prognosis has therefore not been established $^{3,52}$.

Although there are many more claims of prognostic significance of other genetic markers or gene expression in CRC, the question of which marker is strong enough to be applied in daily clinical practice remains unanswered because finding consistent associations between genetic markers and CRC prognosis has proven to be difficult. In addition, questions have arisen as to whether the progression and prognosis of $\mathrm{CRC}$ could be attributed to mutations alone. Because the frequency of mutations in the CAN genes is often low and the majority are missense mutations, it is likely that additional mechanisms contribute to tumor progression and prognosis.

\section{The role of epigenetics}

Epigenetic silencing is a prevalent mechanism that could lead to abnormal gene inactivation in cancer ${ }^{53}$. It has been suggested that aberrant promoter 
$\mathrm{CpG}$ island hypermethylation leading to gene silencing also plays an important role in the neoplastic process, and molecular evidence of this concept has been accumulating since the late $1990 \mathrm{~s}^{54,55}$. Epigenetic alterations may cooperate with genetic abnormalities to affect gene function, eventually leading to cancer ${ }^{56}$. Hypermethylation occurs when DNA methyltransferases (DNMTs) add a methyl group to cytosine to form methyl-cytosine. In humans, these epigenetic alterations include methylation of cytosine located in CpG-islands and may be tissue-specific ${ }^{55,57}$. Methylation within or around the promoter region is associated with gene silencing ${ }^{55}$. Methyl-cytosine-binding proteins and histone deacetylases are recruited to modify nucleosomes, forming transcriptionally repressive chromatin ${ }^{53}$. Traditionally, epigenetic events have primarily been investigated in classic tumor suppressor genes. In this setting, methylation acts as a "second genetic hit" to inactivate the gene ${ }^{53}$. However, more recently, Baylin and co-workers demonstrated that the majority of these genes show promoter $\mathrm{CpG}$ island hypermethylation in addition to the genetic alterations ${ }^{53,58}$. Most mutations found in the CAN genes were heterozygous missense mutations suggesting that these result in haploinsufficiency which is augmented by DNA methylation. In many cases involving tumor suppressor genes with low mutation rates, hypermethylation is a much more frequent event than genetic alterations. This implies that pathway analysis would show that CRCs are not that heterogeneous when both genetic and epigenetic alterations are taken into account ${ }^{53}$.

So far, CRCs have been genetically categorized as either CIN or microsatellite instable (MIN). In addition to these two categories, a subset of CRCs, $17 \%-29 \%{ }^{59-61}$, with a high frequency of $\mathrm{CpG}$-island methylation has recently been described as the CpG-island methylator phenotype (CIMP) ${ }^{59,62-64}$. However, the existence of CIMP and role of CIMP in CRC carcinogenesis has been controversial, mainly due to lack of a generally accepted standard definition and the confounding role of $\mathrm{MSI}$ in determining the CIMP phenotype ${ }^{60,63,65}$. Tumors characterized by CIMP have been associated with $B R A F$ mutations, older age, advanced tumor stage and proximal location ${ }^{26}$. CRCs with less extensive promoter methylation are proposed to be characterized by CIMP-L (CIMP low) and it is hypothesized that these tumors are different compared to CIMP-H (CIMP high) ${ }^{26,63}$. However, evidence is still scarce and this hypothesized distinction between CRCs based on the level of methylation needs further investigation and definition. The distinct molecular genotypes that have been described, CIMP, CIN and MIN, do not seem to be mutually exclusive and thus assessing a single molecular phenotype may not provide all relevant prognostic information ${ }^{35}$. 


\section{Epigenetic markers and CRC prognosis}

The CIMP panel according to Weisenberger et al. ${ }^{59}$ has been associated with poor survival in metastatic MSS CRCs ${ }^{66}$. However, the influence of this panel has not yet been assessed in other stages, and the use of this panel as a prognostic factor remains unresolved. One study reported an association between CIMP (although defined differently, namely using the panel $p 16^{I N K 4 A}$, MINT2 or MDR1) and a worse prognosis among stage III patients treated with surgery alone ${ }^{67}$. In addition, the CIMP panel according to Toyota et al. ${ }^{62}$ was associated with worse survival in stage I-IV CRC patients ${ }^{68}$. This was not observed in another study ${ }^{69}$ suggesting that other epigenetic markers may be responsible for the association. As stated by Ogino and colleagues, additional studies are necessary to further examine the prognostic value of methylation markers among CRC patients in the different stages ${ }^{66}$.

Recently, Chan and colleagues combined information on the methylation of the CAN genes with information from gene expression databases to gain more insight into the prognostic roles of these genes ${ }^{53}$. They analyzed eight genes that were predicted to have a decreased overall expression in CRC by the analysis of microarray data. Two of those genes (PTPRD and RET) were found to be associated with clinical characteristics such as metastasis and grade. However, a number of other CAN genes which were determined to not have altered expression levels related to DNA methylation, no associations with clinical characteristics were found ${ }^{53}$. These results have not yet been validated in other, independent populations.

Despite the growing number of studies on molecular genotypes in CRC, many questions remain unresolved. For example, what is the prognostic relationship of the different genotypes? It is known that they are not mutually exclusive. MIN and CIMP appear to be associated, making the conclusion of whether either one is an independent prognostic factor even more difficult ${ }^{35}$. MSI-H has consistently been associated with better prognosis and decreased metastasis risk $^{70-72}$, although this is often associated with high frequency of $\mathrm{CpG}$ island methylation. The presence and role of MSI-L (MSI low) remains controversial. Mutations often seen in MSI-H appear to be absent in MSI-L and until now, no large differences in clinical or molecular characteristics have been observed between MSI-L and MSS patients ${ }^{26}$. However, reports suggest that MSI-L is indeed a unique subtype of CRC which is associated with loss of $O^{6}-M G M T^{73}$ and related to a poor prognosis in stage III $\mathrm{CRC}^{74}$. Ward et al. showed that DNA methylation in CIMP-associated genes was related to a poor outcome in $\mathrm{CRC}$, but this adverse prognostic effect was lost within those methylated tumors showing $\mathrm{MSI}^{75}$. There seems to be considerable overlap between CIMP and $\mathrm{MSI}$ and it has been suggested that MSI-H has a major effect on the expression of $\mathrm{CIMP}^{60}$ although more recent results imply that $\mathrm{MSI}-\mathrm{H}$ in 
sporadic CRC can be explained by CIMP-H associated MLH1 methylation ${ }^{59}$. These results suggest that MMR deficiency in sporadic CRC is caused by a broad epigenetic control defect affecting MLH1 in most CIMP tumors; an accurate definition of CIMP could therefore be essential in understanding the role of $\mathrm{MSI}-\mathrm{H}^{59}$.

At this moment, reports in the literature are too limited to understand the interplay between $\mathrm{CIN}$ and MIN in CRC prognosis. MSI-H is consistently associated with a better prognosis in $\mathrm{CRC}$, but the underlying explanation for this finding is not yet clear. It has been suggested that this is due to the fact that allelic loss or mutation of $18 \mathrm{q}$, TP53, or KRAS, which are thought to be associated with a poor prognosis, are rare in MSI tumors ${ }^{70}$. However, a recent study on the relation between CIN and MIN concluded that the survival benefit in MSI-H patients was not dependent of CIN status ${ }^{35,44}$ thereby questioning this explanation. In addition, DNA diploidy seemed to be a stronger prognostic factor than MSI in patients treated with 5-FU, and the effect was similar in both $\mathrm{MSI}-\mathrm{H}$ and MSS/MSI-L cases. This could indicate that diploidy may contribute to the often reported better survival in $\mathrm{MSI}-\mathrm{H}$ patients ${ }^{44}$. Nevertheless, diploidy did not seem to be related to treatment response, suggesting that the less aggressive behavior of diploid tumors is not the result of a better treatment response ${ }^{44}$.

\section{Colorectal cancer treatment}

Due to therapeutic innovations, management of advanced CRC has changed considerably in recent years ${ }^{76}$. This has led to an increase in survival, although not substantially. The mainstay of treatment is surgery, with primary tumor, adjacent bowel and lymph nodes removal ${ }^{77}$. Although surgery may be performed for palliative symptom control in advanced cases, in most circumstances it is performed with curative intent. However, with an increasing tumor stage, the chance of cure by surgery decreases. For early-stage CRC (Stage I) only resection is required, without adjuvant therapy ${ }^{77}$. Stage III and IV CRC patients additionally receive adjuvant or palliative chemotherapy. The benefit of adjuvant 5-fluorouracil (5-FU) and leucovorin (LV) has been well established in stage III colon cancers, and is the current standard of care ${ }^{78}$. More recently, oxaliplatin-based chemotherapy, added to regimens containing 5-FU/LV has been shown to further improve disease-free and overall survival in these patients ${ }^{79}$. Other cytostatic agents that have been introduced include DNA topoisomerase I inhibitors, such as irinotecan.

For stage II CRCs, no survival benefit from postoperative chemotherapy in patients with standard risk has been shown in randomised clinical trials, but, despite the initially good prognosis, $20-30 \%$ of these patients will die from CRC 
within 5 years. Unfavourable tumor features in stage II CRCs, such as serosal tumor penetration (T4), tumor perforation, and (extramural) lymphovascular invasion appear to be correlated with an increased risk for recurrence. These features may identify high-risk disease patients, who might benefit from postoperative therapy ${ }^{80}$. Previously, a survival benefit has been suggested for selected stage II tumors after adjuvant chemotherapy ${ }^{81}$.

Recently, therapeutic strategies have also involved the use of humanized monoclonal antibodies, such as bevacizumab and cetuximab. Bevacizumab is a monoclonal antibody directed against vascular endothelial growth factor (VEGF); it improves the outcome of first-line chemotherapy (5-FU/LV) ${ }^{82}$. Cetuximab is a monoclonal antibody directed at the EGFR, and is thought to inhibit signal transduction through this pathway. It is added to chemotherapy with irinotecan (DNA topoisomerase I inhibitor) ${ }^{83}$. Recently, the European Medicine Agency licensed cetuximab and panitumumab, another EGFRtargeted monoclonal antibody, only for patients with metastatic CRC without KRAS mutations after several reports showed an association between KRAS or $B R A F$ mutations and clinical resistance to treatment with EGFR antibodies in metastatic $\mathrm{CRC}^{52,84-88}$. Nevertheless, even among wildtype KRAS CRC patients, response rates for EGFR antibodies are low, ranging from $17 \%$ (for EGFR antibody monotherapy) to $61 \%$ (for EGFR antibody therapy plus oxaliplatin-based chemotherapy). These observations suggest that other mechanisms are involved in therapy resistance. For example, TSER polymorphisms have been implicated as determinants of 5-FU treatment response, with several studies suggesting a poor response to chemotherapy with the 3R/3R genotype ${ }^{89-92}$ although others reported a better response with this genotype ${ }^{46,93}$. Other polymorphisms, such as those in MTHFR and GSTP1 have also been related to treatment response in $C R C$, but evidence is still scarce and inconclusive ${ }^{94}$.

Evidence for a role of $\mathrm{MSI}$ in predicting treatment outcome is also not conclusive. One study has shown that patients with $\mathrm{MSI}-\mathrm{H}$ did not benefit from treatment with 5-FU but MSS or MSI-L tumors did seem to benefit from this treatment ${ }^{95}$. Another study was not able to replicate this finding and even observed an opposite effect in MSI-H tumors that had a greater benefit from 5$\mathrm{FU}^{96}$. With the current evidence, it is not yet possible to draw any conclusions regarding the question of whether $\mathrm{MSI}-\mathrm{H}$ could be used as a predictive marker to distinguish patients that would benefit from 5-FU treatment or not. Given the molecular heterogeneity of the MSI genotype, it may be very difficult to use $\mathrm{MSI}-\mathrm{H}$ as a single predictive marker for CRC treatment ${ }^{26}$.

In addition to clinical response, pharmacogenetic studies have also focussed on the impact of genetic alterations on drug toxicity ${ }^{94,97}$. While these findings are interesting and have led to the first genetic test approved by the U.S. Food 
and Drug Administration, the UGT1A1 test for the prediction of irinotecan toxicity, this topic is beyond the scope of this review.

\section{Implications of epigenetic markers in colorectal cancer treatment}

A critical difference can be made between gene silencing as a result of mutations or aberrant promoter methylation. While mutations are irreversible, epigenetic changes are potentially reversible and therefore present potential new opportunities for cancer treatment. Because of this, studies on epigenetic markers and the prediction of treatment response are gaining increasing attention. For example, loss of $O^{6}-M G M T$ function through hypermethylation is thought to sensitize cells to the effects of chemotherapy based on alkylating mechanisms, since cells have a diminished capacity to repair alkylation damage ${ }^{98}$. Early studies in brain tumors and lymphomas have indeed shown that patients with methylated $O^{6}-M G M T$ respond better to alkylation treatment ${ }^{55}$. In addition, over the last few years, new molecular techniques have been developed which allow researchers to more easily determine DNA methylation. Because of this, DNA-methylation-based technologies have a promising future in both clinical diagnostics and therapeutics ${ }^{99}$. However, results on pharmacoepigenomics are still scarce.

\section{CpG Island methylator phenotype}

The role of aberrant promoter methylation as a predictive marker for treatment response is inconsistent. Although not statistically significant, a trend towards resistance to chemotherapy among CIMP-H tumors has been observed ${ }^{66}$. Furthermore, patients with advanced CRC and hypermethylation of multiple genes, including CIMP-specific genes, have a poor outcome following standard 5 -FU-based chemotherapy ${ }^{100}$. However, these results could not be replicated by another study that showed contrasting data; a survival benefit for CIMP patients after 5 -FU treatment ${ }^{67}$.

Even though the number of studies on the predictive effect of molecular genotypes is slowly increasing, a conclusive answer to the question of whether they are predictive of treatment effect cannot be given at the moment. Further research is needed to resolve this issue. However, although the influence of molecular genotypes on treatment response is one of the main focuses of current research, other markers are potentially important for the future treatment of CRC.

\section{HDAC2}

Disruption of histone acetylation patterns is common in cancer cells and histone modifications that affect chromatin structure are implicated in the inactivation of tumor suppressor genes ${ }^{101,102}$. Recently, truncating mutations in 
one of the human histone deacetylases, HDAC2, were described in cancer cell lines and $\mathrm{CRCs}$ with $\mathrm{MSI}^{102}$. Histone deacetylases maintain gene silencing through histone deacetylation ${ }^{55}$ and are potentially clinically relevant as they might be responsible for the response to HDAC inhibitors; drugs that show promising anticancer activities in clinical trials ${ }^{102,103}$. Indeed, a recent study reported that the presence of a truncating mutation in HDAC2 leads to a loss of protein expression and consequently more resistance to the antiproliferative and proapoptotic effects of HDAC inhibitors ${ }^{102}$. This report also suggested that HDAC2-deficiency confers resistance to TSA-induced histone acetylation, but this could not be confirmed by others ${ }^{104}$. Furthermore, it has been demonstrated that cells lacking HDAC2 are not able to induce APAF1, which appears to be the key to apoptosis induced by HDAC inhibitors ${ }^{105}$. These recent findings support the use of $H D A C 2$ mutational status as a guide for the prescription of $H D A C$ inhibitors in CRC. However, literature on this topic is still limited and more research on the potential use of HDAC2 as a marker in CRC is needed.

\section{Werner (WRN)}

Other epigenetic markers, such as those associated with metastasis, have been suggested to act as prognostic or predictive markers ${ }^{106}$. One of these is promoter CpG island methylation of the Werner syndrome gene (WRM), which has been associated with longer survival of patients treated with chemotherapy ${ }^{107}$. The WRN gene and its protein are involved in the maintenance of telomere structure and initiation of DNA damage response after telomere disruption ${ }^{108}$. As it was observed that patients with WRN germline mutations often develop epithelial or mesenchymal tumors, it was suggested that the WRN gene had a tumor-suppressor function ${ }^{107}$. It has been demonstrated that WRN is inactivated due to hypermethylation in human cancer cells. Inactivation of WRN leads to hypersensitivity to topoisomerase inhibitors, such as irinotecan which is often used in the treatment of CRC, and DNA-damaging agents ${ }^{107}$. Indeed, recent studies have observed an increased survival in patients with WRN methylation after treatment with a topoisomerase inhibitor ${ }^{107}$. In addition, WRN methylation appears to be associated with mucinous differentiation in colorectal tumors independent of MSI or CIMP ${ }^{108}$. Mucinous differentiation is also suggested to be associated with other molecular tumor characteristics, including MSI-H, BRAF mutations, KRAS mutations and loss of $T P 53^{108}$. This raises questions of whether WRN, in addition to its possible role as predictive marker for treatment response, could also have a prognostic influence in CRC. Moreover, the observation that WRN methylation is associated with increased survival in CRC after treatment with irinotecan needs further exploration and confirmation in other larger populations before this marker can be used. 


\section{CHFR}

Recently, CHFR was identified as a mitotic checkpoint gene, located on chromosome 12q24.33 ${ }^{109}$. CHFR functions early in the G2/M transition and mediates a delay of entry into metaphase in response to mitotic stress induced by nocodazole or paclitaxel. CHFR functions as a tumor suppressor and several groups have demonstrated that CHFR expression is lost or decreased in most human cancers, mostly due to DNA promoter hypermethylation. Epigenetic loss of CHFR expression is however rare in primary breast cancer ${ }^{110,111}$. In endometrial and cervical cancer, it has been shown that DNA hypermethylation of $C H F R$ predisposes cancer cells to a higher sensitivity to taxanes. Cell lines hypermethylated for CHFR with high sensitivity to taxanes became resistant after demethylation ${ }^{112,113}$. It has been suggested that $C H F R$ methylation status could act as a new molecular index, allowing for the design of personalized treatment in endometrial cancer. In advanced gastric cancer however, CHFR hypermethylation is not consistently associated with response to docetaxel and paclitaxel, both of which are taxanes ${ }^{114,115}$.

While taxanes are not used in the adjuvant treatment setting of CRC, because they failed to demonstrate a significant clinical benefit in phase II trials in $\mathrm{CRC}^{116}$, frequent hypermethylation of $C H F R$ is detected in CRC. Approximately $37 \%$ of all primary CRCs are hypermethylated for $C H F R^{117,118}$, raising the possibility that CHFR methylation could be used in CRC to identify those patients who might benefit from taxanes.

Although application of these molecular markers is not yet accepted in standard patient care, they serve as examples of a shifting paradigm, drawing on the notion that awareness of molecular alterations in tumors can be harnessed for more selective and effective treatment schedules. It is both conceivable and desirable that molecular profiling will more accurately identify those patients with a greater chance of benefitting from adjuvant treatment. These predictive molecular profiles will help define the best way to integrate chemotherapy with biology.

\section{Future perspectives}

Recently, increasing attention has been given to the role of genetic and epigenetic alterations in attempting to answer the question of which CRC patients should receive (adjuvant) treatment and which treatment is most suitable for an individual patient. Numerous studies have been published in the last decade discussing the influence of (epi)genetic markers on CRC prognosis and treatment outcome prediction.

Although epigenetic alterations are heritable in somatic cells, they could potentially be reversed by drug treatment; a characteristic that is not shared by genetic alterations. This could have significant implications for the treatment of 
CRC. The recent publications on the use of HDAC inhibitors in clinical trials yielding promising results, suggest that the use of epigenetic therapy is promising. In addition, epigenetic alterations are frequent in CRC, even more frequent than genetic alterations, and recent new molecular techniques enable researchers to easily assess methylation status and identify novel epigenetic markers. Because of this, it is conceivable that epigenetic markers will eventually have a high impact, maybe even higher than to genetic markers, on the management of CRC.

Figure 2.1 provides an overview of the current knowledge on (epi)genetic markers discussed in this review and their role in prognosis and treatment prediction. A more extensive staging system based on (epi)genetic markers will have more impact on stage II and III patients, as it is often not clear which patients to treat or which treatment to prescribe to these patients. Although many studies have led to the overview in Figure 2.1, results are often inconsistent and many findings have not yet been replicated in other, independent populations. Because of this, it is difficult to draw definitive conclusions on the prognostic or predictive role of these (epi)genetic markers.

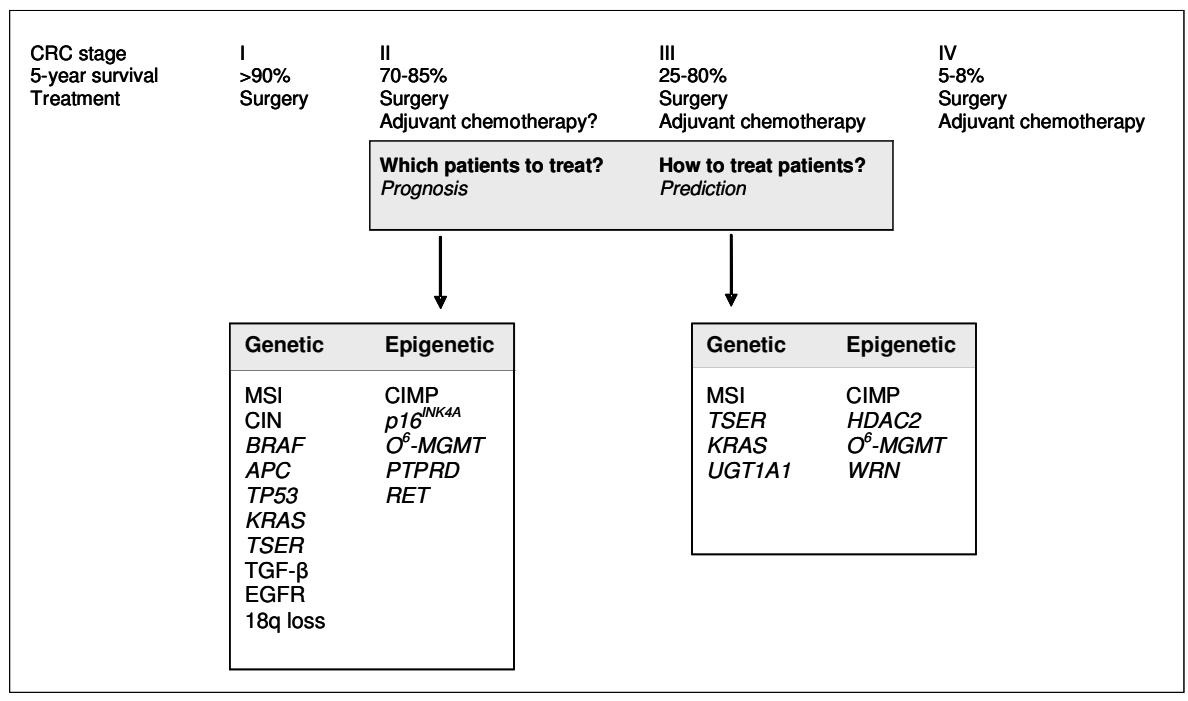

Figure 2.1 Current knowledge on (epi)genetic markers and prognosis / prediction 


\section{High-throughput technologies and novel approaches}

In the last few years, an increasing number of molecular techniques to detect DNA methylation on a larger scale has been reported. Following the development of bisulfite sequencing ${ }^{119}$, rapid progress has been made in the characterization of the methylation state of individual cytosines. However, as this involves locus-specific amplification, it is challenging to use on a large scale $^{120}$. Bisulfite-conversion based microarrays and high-throughput PCR sequencing approaches, and more recently, next-generation bisulfite sequencing ${ }^{121}$ have been developed in an attempt to use this approach on a larger scale. In 2002, Suzuki and colleagues described a new microarrayapproach $^{122}$, a method which was recently adapted by Schuebel et al. ${ }^{53,58,122}$. This approach has proven to be successful in identifying genes silenced by hypermethylation which can subsequently be studied for prognostic influence as described by Chan and colleagues ${ }^{53}$. Methylated $\mathrm{CpG}$ island amplification (MCA) coupled with Representational Difference Analysis (RDA) has been described as a tool to identify methylation at multiple loci ${ }^{123}$. Alternatively, immunoprecipitation-based methods (ChIP) can be used for distinguishing between methylated and unmethylated fractions ${ }^{120,124,125}$. Other methods either use 5-methylcytosine-specific antibodies (methylated DNA immunoprecipitation, MeDIP or MDIP) or methyl-binding domain proteins to enrich for the methylated fraction of the genome ${ }^{121}$. Indirect approaches for DNA methylation profiling on array platforms have also been developed which are based on the high affinity of some proteins to methylated DNA. This technique, the methylated-CpG island recovery assay (MIRA), has already been used in a genome-wide screen to identify DNA methylation markers ${ }^{125,126}$. Although many methylation-associated gene silencing events in human cancer have been studied, little is known about why some $\mathrm{CpG}$ islands are methylated in cancer whereas others seem resistant to this de novo methylation. In 2003, Feltus and colleagues described that not all CpG islands are equally affected ${ }^{127}$. Using DNA pattern recognition and supervised learning techniques, they obtained a classification function that could be used to distinguish $\mathrm{CpG}$ islands sensitive to methylation ${ }^{127}$. Other techniques also make use of a bioinformatics approach. In 2002, several tumor suppressor gene candidates in esophageal squamous cell carcinoma were identified using a technique based on functional reactivation of epigenetically silenced tumor suppressor genes by 5-aza-2'deoxycytidine and TSA in combination with an intuitive algorithm ${ }^{128}$. Recently, next generation sequencing technologies processing millions of sequence reads in parallel, have emerged as powerful tools for whole genome profiling of epigenetic modifications. Next generation sequencing technologies have successfully been combined with ChIP techniques (ChIP-seq) ${ }^{129}$. These studies demonstrate that different genome-wide approaches can successfully 
Pharmacoepigenomics in colorectal cancer $\mid 35$

be combined in order to gain more insight and knowledge on cancer epigenomics.

\section{Conclusion}

Recent innovations suggest that the current technology could indeed lead to the knowledge necessary to enable the implementation of epigenetic therapies in everyday practice. However, from a scientific point of view, many questions remain unresolved and although epigenetic alterations seem to be promising prognostic and predictive markers for $\mathrm{CRC}$, some issues should be taken into account when assessing the clinical use of pharmaco(epi)genomics. Despite numerous studies on the relevance of molecular markers in oncology, the number of clinically useful markers is limited ${ }^{130}$. Research on molecular markers is susceptible to publication bias and false-positive results, due to small population sizes and small effect sizes ${ }^{131-133}$. Often, an initial study shows promising results that cannot be replicated in later studies ${ }^{130,131}$. Moreover, the majority of previous studies describe single molecular markers ${ }^{3}$, even though it is suggested that using an approach of combining several markers is more likely to identify relevant prognostic factors ${ }^{134}$. The available evidence on the prognostic or predictive influence of molecular markers is limited, even though this question has received much attention. It is likely that epigenetic alterations play a role in CRC prognosis or treatment response, however, it is not clear to what extent, and if this role is independent of other known prognostic factors, such as tumor stage. To answer these questions and to maximize the potential of the novel technologies, the American Association for Cancer Research Human Epigenome Task Force and the Scientific Advisory Board of the Epigenome Network of Excellence from the European Union recently urged the scientific community to collaborate and join the Alliance for the Human Epigenome and Disease (AHEAD) in order to help solve the problem of cancer $^{135}$. AHEAD is aiming to define the patterns of epigenetic regulation occurring in different cell states and therefore complements other ongoing projects such as ENCODE that aim to define the functional sequences in the genome $^{135}$.

More research is necessary to assess the prognostic and predictive influence of epigenetic markers in CRC and consequently, to determine the clinical use of such findings. However, existing technologies provide us with the tools so that in time, we will be able to fill all the voids in our current understanding of pharmacoepigenomics in CRC and we will be capable to accurately answer the questions of which CRC patients are in need of a more aggressive treatment, and which treatment is most suitable for an individual patient. 


\section{References}

1. Klump B, Nehls $\mathrm{O}$, Okech $\mathrm{T}$, et al. Molecular lesions in colorectal cancer: impact on prognosis? Original data and review of the literature. Int J Colorectal Dis 19(1):23-42, 2004

2. Graziano F, Cascinu S. Prognostic molecular markers for planning adjuvant chemotherapy trials in Dukes' B colorectal cancer patients: how much evidence is enough? Ann Oncol 14(7):1026-38, 2003

3. McLeod HL, Murray Gl. Tumour markers of prognosis in colorectal cancer. $\mathrm{Br} \mathrm{J}$ Cancer 79(2):191-203, 1999

4. Ferlay J, Autier P, Boniol M, et al. Estimates of the cancer incidence and mortality in Europe in 2006. Ann Oncol 18(3):581-92, 2007

5. GLOBOCAN I. Cancer Incidence Mortality and Prevalence Worldwide. 2002

6. WCRF, AICR. Food, Nutrition, Physical Activity, and the Prevention of Cancer: a Global Perspective. Washington DC: AICR, 2007

7. Mariotto $\mathrm{AB}$, Rowland $\mathrm{JH}$, Ries $\mathrm{LA}$, et al. Multiple cancer prevalence: a growing challenge in long-term survivorship. Cancer Epidemiol Biomarkers Prev 16(3):566-71, 2007

8. Seeff LC, Richards TB, Shapiro JA, et al. How many endoscopies are performed for colorectal cancer screening? Results from CDC's survey of endoscopic capacity. Gastroenterology 127(6):1670-7, 2004

9. Bressler B, Lo C, Amar J, et al. Prospective evaluation of screening colonoscopy: who is being screened? Gastrointest Endosc 60(6):921-6, 2004

10. Whitney D, Skoletsky J, Moore K, et al. Enhanced retrieval of DNA from human fecal samples results in improved performance of colorectal cancer screening test. J Mol Diagn 6(4):386-95, 2004

11. Levin B, Lieberman DA, McFarland B, et al. Screening and surveillance for the early detection of colorectal cancer and adenomatous polyps, 2008: a joint guideline from the American Cancer Society, the US Multi-Society Task Force on Colorectal Cancer, and the American College of Radiology. Gastroenterology 134(5):1570-95, 2008

12. Huang Z, Li L, Wang J. Hypermethylation of SFRP2 as a potential marker for stool-based detection of colorectal cancer and precancerous lesions. Dig Dis Sci 52(9):2287-91, 2007

13. Wang DR, Tang D. Hypermethylated SFRP2 gene in fecal DNA is a high potential biomarker for colorectal cancer noninvasive screening. World J Gastroenterol 14(4):524-31, 2008

14. Hamilton SR, A.Aaltonen L, eds. Pathology \& genetics of Tumours of the Digestive System. World Health Organization of the Digestive System. Lyon, France: International Agency for Research on Cancer, 2000.

15. Compton CC, Greene FL. The staging of colorectal cancer: 2004 and beyond. CA Cancer J Clin 54(6):295-308, 2004

16. Vogelstein B, Bearon ER, Hamilton SR. Genetic alterations during colorectal-tumor development. New England Journal of Medicine 319:525-32, 1988

17. Fearon ER, Vogelstein B. A genetic model for colorectal tumorigenesis. Cell 61(5):759-67, 1990

18. Powell SM, Zilz N, Beazer-Barclay $\mathrm{Y}$, et al. APC mutations occur early during colorectal tumorigenesis. Nature 359(6392):235-7, 1992

19. Groden J, Thliveris A, Samowitz W, et al. Identification and characterization of the familial adenomatous polyposis coli gene. Cell 66(3):589-600, 1991

20. Tomlinson I, llyas M, Novelli M. Molecular genetics of colon cancer. Cancer Metastasis Rev 16(1-2):67-79, 1997

21. Kolligs FT, Bommer G, Goke B. Wnt/beta-catenin/tcf signaling: a critical pathway in gastrointestinal tumorigenesis. Digestion 66(3):131-44, 2002

22. Janssen KP, Alberici $P$, Fsihi $H$, et al. APC and oncogenic KRAS are synergistic in enhancing Wnt signaling in intestinal tumor formation and progression. Gastroenterology 131(4):1096109, 2006

23. Fodde R. The APC gene in colorectal cancer. Eur J Cancer 38(7):867-71, 2002 
Pharmacoepigenomics in colorectal cancer $\mid 37$

24. Houlston RS, Tomlinson IP. Genetic prognostic markers in colorectal cancer. Mol Pathol 50(6):281-8, 1997

25. Miranda E, Destro A, Malesci A, et al. Genetic and epigenetic changes in primary metastatic and nonmetastatic colorectal cancer. Br J Cancer 95(8):1101-7, 2006

26. Imai $\mathrm{K}$, Yamamoto $\mathrm{H}$. Carcinogenesis and microsatellite instability: the interrelationship between genetics and epigenetics. Carcinogenesis 29(4):673-80, 2008

27. Fearon ER, Cho KR, Nigro JM, et al. Identification of a chromosome 18q gene that is altered in colorectal cancers. Science 247(4938):49-56, 1990

28. Thibodeau SN, Bren G, Schaid D. Microsatellite instability in cancer of the proximal colon. Science 260(5109):816-9, 1993

29. Miyakura $Y$, Sugano K, Konishi F, et al. Methylation profile of the MLH1 promoter region and their relationship to colorectal carcinogenesis. Genes Chromosomes Cancer 36(1):17-25, 2003

30. Leslie A, Carey FA, Pratt NR, Steele RJ. The colorectal adenoma-carcinoma sequence. Br J Surg 89(7):845-60, 2002

31. Sjoblom T, Jones S, Wood LD, et al. The consensus coding sequences of human breast and colorectal cancers. Science 314(5797):268-74, 2006

32. Wood LD, Parsons DW, Jones S, et al. The genomic landscapes of human breast and colorectal cancers. Science 318(5853):1108-13, 2007

33. Vogelstein B, Kinzler KW. Cancer genes and the pathways they control. Nat Med 10(8):78999, 2004

34. Kinzler KW, Vogelstein B. Lessons from hereditary colorectal cancer. Cell 87(2):159-70, 1996

35. Walther A, Houlston R, Tomlinson I. Association between chromosomal instability and prognosis in colorectal cancer: a meta-analysis. Gut 57(7):941-50, 2008

36. Locker GY, Hamilton S, Harris J, et al. ASCO 2006 update of recommendations for the use of tumor markers in gastrointestinal cancer. J Clin Oncol 24(33):5313-27, 2006

37. Castagnola P, Giaretti W. Mutant KRAS, chromosomal instability and prognosis in colorectal cancer. Biochim Biophys Acta 1756(2):115-25, 2005

38. Anwar S, Frayling IM, Scott NA, Carlson GL. Systematic review of genetic influences on the prognosis of colorectal cancer. Br J Surg 91(10):1275-91, 2004

39. Westra JL, Plukker JT, Buys $\mathrm{CH}$, et al. Genetic alterations in locally advanced stage II/III colon cancer: a search for prognostic markers. Clin Colorectal Cancer 4(4):252-9, 2004

40. Samowitz WS, Sweeney C, Herrick J, et al. Poor survival associated with the BRAF V600E mutation in microsatellite-stable colon cancers. Cancer Res 65(14):6063-9, 2005

41. Lanza G, Matteuzzi M, Gafa R, et al. Chromosome 18q allelic loss and prognosis in stage II and III colon cancer. Int J Cancer 79(4):390-5, 1998

42. Jen J, Kim H, Piantadosi S, et al. Allelic loss of chromosome $18 \mathrm{q}$ and prognosis in colorectal cancer. N Engl J Med 331(4):213-21, 1994

43. Postma C, Terwischa S, Hermsen MA, et al. Gain of chromosome 20q is an indicator of poor prognosis in colorectal cancer. Cell Oncol 29(1):73-5, 2007

44. Sinicrope FA, Rego RL, Halling KC, et al. Prognostic impact of microsatellite instability and DNA ploidy in human colon carcinoma patients. Gastroenterology 131(3):729-37, 2006

45. Ulrich CM, Curtin K, Potter JD, et al. Polymorphisms in the reduced folate carrier, thymidylate synthase, or methionine synthase and risk of colon cancer. Cancer Epidemiol Biomarkers Prev 14(11 Pt 1):2509-16, 2005

46. Dotor E, Cuatrecases M, Martinez-Iniesta M, et al. Tumor thymidylate synthase 1494 del6 genotype as a prognostic factor in colorectal cancer patients receiving fluorouracil-based adjuvant treatment. J Clin Oncol 24(10):1603-11, 2006

47. Chen J, Hunter DJ, Stampfer MJ, et al. Polymorphism in the thymidylate synthase promoter enhancer region modifies the risk and survival of colorectal cancer. Cancer Epidemiol Biomarkers Prev 12(10):958-62, 2003

48. Curtin K, Ulrich CM, Samowitz WS, et al. Thymidylate synthase polymorphisms and colon cancer: associations with tumor stage, tumor characteristics and survival. Int $\mathrm{J}$ Cancer 120(10):2226-32, 2007 
38 $\mid$ Chapter 2

49. Bacman D, Merkel S, Croner R, et al. TGF-beta receptor 2 downregulation in tumourassociated stroma worsens prognosis and high-grade tumours show more tumour-associated macrophages and lower TGF-beta1 expression in colon carcinoma: a retrospective study. BMC Cancer ;7:156, 2007

50. Robson $\mathrm{H}$, Anderson $\mathrm{E}$, James RD, et al. Transforming growth factor beta 1 expression in human colorectal tumours: an independent prognostic marker in a subgroup of poor prognosis patients. Br J Cancer 74(5):753-8, 1996

51. Kouraklis G, Kakisis J, Theoharis $\mathrm{S}$, et al. Prognostic significance and correlation with survival of bcl-2 and TGF-beta RII in colon cancer. Dig Dis Sci 48(12):2284-9, 2003

52. Spano JP, Milano G, Vignot $\mathrm{S}$, et al. Potential predictive markers of response to EGFRtargeted therapies in colorectal cancer. Crit Rev Oncol Hematol 66(1):21-30, 2008

53. Chan TA, Glockner S, Yi JM, et al. Convergence of mutation and epigenetic alterations identifies common genes in cancer that predict for poor prognosis. PLoS Med 5(5):e114, 2008

54. Herman JG. Hypermethylation of tumor suppressor genes in cancer. Semin Cancer Biol 9(5):359-67, 1999

55. Herman JG, Baylin SB. Gene silencing in cancer in association with promoter hypermethylation. N Engl J Med 349(21):2042-54, 2003

56. Jones PA, Baylin SB. The epigenomics of cancer. Cell 128(4):683-92, 2007

57. Esteller M, Herman JG. Cancer as an epigenetic disease: DNA methylation and chromatin alterations in human tumours. J Pathol 196(1):1-7, 2002

58. Schuebel KE, Chen W, Cope L, et al. Comparing the DNA hypermethylome with gene mutations in human colorectal cancer. PLoS Genet 3(9):1709-23, 2007

59. Weisenberger DJ, Siegmund KD, Campan M, et al. CpG island methylator phenotype underlies sporadic microsatellite instability and is tightly associated with BRAF mutation in colorectal cancer. Nat Genet 38(7):787-93, 2006

60. Samowitz WS, Albertsen H, Herrick J, et al. Evaluation of a large, population-based sample supports a CpG island methylator phenotype in colon cancer. Gastroenterology 129(3):83745, 2005

61. Ogino S, Odze RD, Kawasaki T, et al. Correlation of pathologic features with $\mathrm{CpG}$ island methylator phenotype (CIMP) by quantitative DNA methylation analysis in colorectal carcinoma. Am J Surg Pathol 30(9):1175-83, 2006

62. Toyota M, Ahuja N, Ohe-Toyota M, et al. CpG island methylator phenotype in colorectal cancer. Proc Natl Acad Sci U S A 96(15):8681-6, 1999

63. Shen L, Toyota M, Kondo $\mathrm{Y}$, et al. Integrated genetic and epigenetic analysis identifies three different subclasses of colon cancer. Proc Natl Acad Sci U S A 104(47):18654-9, 2007

64. Ogino S, Goel A. Molecular Classification and Correlates in Colorectal Cancer. J Mol Diagn 10(1):13-27, 2008

65. Issa JP. CpG island methylator phenotype in cancer. Nat Rev Cancer 4(12):988-93, 2004

66. Ogino S, Meyerhardt JA, Kawasaki T, et al. CpG island methylation, response to combination chemotherapy, and patient survival in advanced microsatellite stable colorectal carcinoma. Virchows Arch 2007.

67. Van Rijnsoever M, Elsaleh $H$, Joseph $D$, et al. CpG island methylator phenotype is an independent predictor of survival benefit from 5 -fluorouracil in stage III colorectal cancer. Clin Cancer Res 9(8):2898-903, 2003

68. Hawkins N, Norrie M, Cheong K, et al. CpG island methylation in sporadic colorectal cancers and its relationship to microsatellite instability. Gastroenterology 122(5):1376-87, 2002

69. van Rijnsoever M, Grieu F, Elsaleh H, et al. Characterisation of colorectal cancers showing hypermethylation at multiple CpG islands. Gut 51(6):797-802, 2002

70. Popat S, Hubner R, Houlston RS. Systematic review of microsatellite instability and colorectal cancer prognosis. J Clin Oncol 23(3):609-18, 2005

71. Wright $\mathrm{CM}$, Dent $\mathrm{OF}$, Barker M, et al. Prognostic significance of extensive microsatellite instability in sporadic clinicopathological stage C colorectal cancer. Br J Surg 87(9):1197-202, 2000 
Pharmacoepigenomics in colorectal cancer

72. Gryfe R, Kim H, Hsieh ET, et al. Tumor microsatellite instability and clinical outcome in young patients with colorectal cancer. N Engl J Med 342(2):69-77, 2000

73. Whitehall VL, Walsh MD, Young $\mathrm{J}$, et al. Methylation of O-6-methylguanine DNA methyltransferase characterizes a subset of colorectal cancer with low-level DNA microsatellite instability. Cancer Res 61(3):827-30, 2001

74. Kohonen-Corish MR, Daniel JJ, Chan C, et al. Low microsatellite instability is associated with poor prognosis in stage $\mathrm{C}$ colon cancer. J Clin Oncol 23(10):2318-24, 2005

75. Ward RL, Cheong K, Ku SL, Meagher A, et al. Adverse prognostic effect of methylation in colorectal cancer is reversed by microsatellite instability. J Clin Oncol 21(20):3729-36, 2003

76. Saunders M, Iveson T. Management of advanced colorectal cancer: state of the art. $\mathrm{Br} J$ Cancer 95(2):131-8, 2006

77. Nelson H, Petrelli N, Carlin A, et al. Guidelines 2000 for colon and rectal cancer surgery. J Natl Cancer Inst 93(8):583-96, 2001

78. Loupakis F, Masi G, Vasile E, et al. First-line chemotherapy in metastatic colorectal cancer: new approaches and therapeutic algorithms. Always hit hard first? Curr Opin Oncol 20(4):459-65, 2008

79. Taieb J, Puig PL, Bedenne L. Cetuximab plus FOLFOX-4 for fully resected stage III colon carcinoma: scientific background and the ongoing PETACC-8 trial. Expert Rev Anticancer Ther 8(2):183-9, 2008

80. Wolpin BM, Mayer RJ. Systemic treatment of colorectal cancer. Gastroenterology 134(5):1296-310, 2008

81. Moertel CG, Fleming TR, Macdonald JS, et al. Levamisole and fluorouracil for adjuvant therapy of resected colon carcinoma. N Engl J Med 322(6):352-8, 1990

82. Tabernero J, Van Cutsem E, Diaz-Rubio E, et al. Phase II trial of cetuximab in combination with fluorouracil, leucovorin, and oxaliplatin in the first-line treatment of metastatic colorectal cancer. J Clin Oncol 25(33):5225-32, 2007

83. Kabbinavar FF, Hambleton J, Mass RD, et al. Combined analysis of efficacy: the addition of bevacizumab to fluorouracil/leucovorin improves survival for patients with metastatic colorectal cancer. J Clin Oncol 23(16):3706-12, 2005

84. Moroni M, Veronese S, Benvenuti S, et al. Gene copy number for epidermal growth factor receptor (EGFR) and clinical response to antiEGFR treatment in colorectal cancer: a cohort study. Lancet Oncol 6(5):279-86, 2005

85. Lievre $\mathrm{A}$, Bachet JB, Le Corre $\mathrm{D}$, et al. KRAS mutation status is predictive of response to cetuximab therapy in colorectal cancer. Cancer Res 66(8):3992-5, 2006

86. Benvenuti S, Sartore-Bianchi A, Di Nicolantonio F, et al. Oncogenic activation of the RAS/RAF signaling pathway impairs the response of metastatic colorectal cancers to antiepidermal growth factor receptor antibody therapies. Cancer Res 67(6):2643-8, 2007

87. Khambata-Ford S, Garrett CR, Meropol NJ,et al. Expression of epiregulin and amphiregulin and K-ras mutation status predict disease control in metastatic colorectal cancer patients treated with cetuximab. J Clin Oncol 25(22):3230-7, 2007

88. Di Fiore F, Blanchard F, Charbonnier F, et al. Clinical relevance of KRAS mutation detection in metastatic colorectal cancer treated by Cetuximab plus chemotherapy. $\mathrm{Br} \mathrm{J}$ Cancer 96(8):1166-9, 2007

89. lacopetta B, Grieu F, Joseph D, et al. A polymorphism in the enhancer region of the thymidylate synthase promoter influences the survival of colorectal cancer patients treated with 5-fluorouracil. Br J Cancer 85(6):827-30, 2001

90. Villafranca E, Okruzhnov Y, Dominguez MA, et al. Polymorphisms of the repeated sequences in the enhancer region of the thymidylate synthase gene promoter may predict downstaging after preoperative chemoradiation in rectal cancer. J Clin Oncol 19(6):1779-86, 2001

91. Marsh S. Thymidylate synthase pharmacogenetics. Invest New Drugs 23(6):533-7, 2005

92. Pullarkat ST, Stoehlmacher J, Ghaderi V, et al. Thymidylate synthase gene polymorphism determines response and toxicity of 5-FU chemotherapy. Pharmacogenomics J 1(1):65-70, 2001 
$40 \mid$ Chapter 2

93. Jakobsen A, Nielsen JN, Gyldenkerne N, et al. Thymidylate synthase and methylenetetrahydrofolate reductase gene polymorphism in normal tissue as predictors of fluorouracil sensitivity. J Clin Oncol 23(7):1365-9, 2005

94. Bandres E, Zarate R, Ramirez N, et al. Pharmacogenomics in colorectal cancer: the first step for individualized-therapy. World J Gastroenterol 13(44):5888-901, 2007

95. Ribic CM, Sargent DJ, Moore MJ, et al. Tumor microsatellite-instability status as a predictor of benefit from fluorouracil-based adjuvant chemotherapy for colon cancer. $\mathrm{N}$ Engl J Med 349(3):247-57, 2003

96. Elsaleh $\mathrm{H}$, Powell $\mathrm{B}$, Soontrapornchai $\mathrm{P}$, et al. p53 gene mutation, microsatellite instability and adjuvant chemotherapy: impact on survival of 388 patients with Dukes' $\mathrm{C}$ colon carcinoma. Oncology 58(1):52-9, 2000

97. Funke S, Brenner H, Chang-Claude J. Pharmacogenetics in colorectal cancer: a systematic review. Pharmacogenomics 9(8):1079-99, 2008

98. Esteller M, Herman JG. Generating mutations but providing chemosensitivity: the role of O6methylguanine DNA methyltransferase in human cancer. Oncogene 23(1):1-8, 2004

99. Laird PW. The power and the promise of DNA methylation markers. Nat Rev Cancer 3(4):253-66, 2003

100. Shen L, Catalano PJ, Benson AB, et al. Association between DNA methylation and shortened survival in patients with advanced colorectal cancer treated with 5-fluorouracil based chemotherapy. Clin Cancer Res 13(20):6093-8, 2007

101. Konishi K, Issa JP. Targeting aberrant chromatin structure in colorectal carcinomas. Cancer J 13(1):49-55, 2007

102. Ropero S, Fraga MF, Ballestar E, et al. A truncating mutation of HDAC2 in human cancers confers resistance to histone deacetylase inhibition. Nat Genet 38(5):566-9, 2006

103. Marks PA, Jiang $X$. Histone deacetylase inhibitors in programmed cell death and cancer therapy. Cell Cycle 4(4):549-51, 2005

104. Ree AH, Folkvord S, Flatmark K. HDAC2 deficiency and histone acetylation. Nat Genet 40(7):812-3, 2008

105. Hanigan $\mathrm{CL}$, van Engeland $\mathrm{M}$, de Bruïne $\mathrm{AP}$, et al. An inactivating mutation in HDAC2 leads to a dysregulation of apoptosis mediated by APAF1. Gastroenterology 135:1654-1664, 2008

106. Lyall MS, Dundas SR, Curran S, et al. Profiling markers of prognosis in colorectal cancer. Clin Cancer Res 12(4):1184-91, 2006

107. Agrelo R, Cheng WH, Setien $F$, et al. Epigenetic inactivation of the premature aging Werner syndrome gene in human cancer. Proc Natl Acad Sci U S A 103(23):8822-7, 2006

108. Kawasaki T, Ohnishi M, Suemoto $\mathrm{Y}$, et al. WRN promoter methylation possibly connects mucinous differentiation, microsatellite instability and $\mathrm{CpG}$ island methylator phenotype in colorectal cancer. Mod Pathol 21(2):150-8, 2008

109. Scolnick DM, Halazonetis TD. Chfr defines a mitotic stress checkpoint that delays entry into metaphase. Nature 406(6794):430-5, 2000

110. Toyota M, Sasaki Y, Satoh A, et al. Epigenetic inactivation of CHFR in human tumors. Proc Natl Acad Sci U S A 100(13):7818-23, 2003

111. Tokunaga E, Oki E, Nishida K, et al. Aberrant hypermethylation of the promoter region of the CHFR gene is rare in primary breast cancer. Breast Cancer Res Treat 97(2):199-203, 2006

112. Yanokura M, Banno K, Kawaguchi M, et al. Relationship of aberrant DNA hypermethylation of CHFR with sensitivity to taxanes in endometrial cancer. Oncol Rep 17(1):41-8, 2007

113. Banno K, Yanokura M, Kawaguchi M, et al. Epigenetic inactivation of the CHFR gene in cervical cancer contributes to sensitivity to taxanes. Int J Oncol 31(4):713-20, 2007

114. Yoshida K, Hamai Y, Suzuki T, et al. DNA methylation of CHFR is not a predictor of the response to docetaxel and paclitaxel in advanced and recurrent gastric cancer. Anticancer Res 26(1A):49-54, 2006

115. Satoh A, Toyota $M$, Itoh $F$, et al. Epigenetic inactivation of CHFR and sensitivity to microtubule inhibitors in gastric cancer. Cancer Res 63(24):8606-13, 2003

116. Swanton C, Tomlinson I, Downward J. Chromosomal instability, colorectal cancer and taxane resistance. Cell Cycle 5(8):818-23, 2006 
117. Corn PG, Summers MK, Fogt $F$, et al. Frequent hypermethylation of the $5^{\prime} \mathrm{CpG}$ island of the mitotic stress checkpoint gene Chfr in colorectal and non-small cell lung cancer. Carcinogenesis 24(1):47-51, 2003

118. Brandes JC, van Engeland M, Wouters KA, et al. CHFR promoter hypermethylation in colon cancer correlates with the microsatellite instability phenotype. Carcinogenesis 26(6):1152-6, 2005

119. Clark SJ, Harrison J, Paul CL, et al. High sensitivity mapping of methylated cytosines. Nucleic Acids Res 22(15):2990-7, 1994

120. Bernstein BE, Meissner A, Lander ES. The mammalian epigenome. Cell 128(4):669-81, 2007

121. Down TA, Rakyan VK, Turner DJ, et al. A Bayesian deconvolution strategy for immunoprecipitation-based DNA methylome analysis. Nat Biotechnol 26(7):779-85, 2008

122. Suzuki H, Gabrielson E, Chen W, et al. A genomic screen for genes upregulated by demethylation and histone deacetylase inhibition in human colorectal cancer. Nat Genet 31(2):141-9, 2002

123. Chung W, Kwabi-Addo B, Ittmann M, et al. Identification of novel tumor markers in prostate, colon and breast cancer by unbiased methylation profiling. PLoS ONE 3(4):e2079, 2008

124. Wilson IM, Davies JJ, Weber M, et al. Epigenomics: mapping the methylome. Cell Cycle 5(2):155-8, 2006

125. Beck S, Rakyan VK. The methylome: approaches for global DNA methylation profiling. Trends Genet 24(5):231-7, 2008

126. Rauch $\mathrm{T}$, Li H, Wu X, et al. MIRA-assisted microarray analysis, a new technology for the determination of DNA methylation patterns, identifies frequent methylation of homeodomaincontaining genes in lung cancer cells. Cancer Res 66(16):7939-47, 2006

127. Feltus FA, Lee EK, Costello JF, et al. Predicting aberrant $\mathrm{CpG}$ island methylation. Proc Natl Acad Sci U S A 100(21):12253-8, 2003

128. Yamashita K, Upadhyay S, Osada M, et al. Pharmacologic unmasking of epigenetically silenced tumor suppressor genes in esophageal squamous cell carcinoma. Cancer Cell 2(6):485-95, 2002

129. Barski A, Cuddapah S, Cui K, et al. High-resolution profiling of histone methylations in the human genome. Cell 129(4): 823-37, 2007

130. McShane LM, Altman DG, Sauerbrei W, Taube SE, Gion M, Clark GM. Reporting recommendations for tumor marker prognostic studies (REMARK). J Natl Cancer Inst 97(16): 1180-4, 2005

131. Ioannidis JP. Is molecular profiling ready for use in clinical decision making? Oncologist 12(3):301-11, 2007

132. Ioannidis JP. Why most published research findings are false. PLoS Med 2(8):e124, 2005

133. Ioannidis JP. Molecular bias. Eur J Epidemiol 20(9):739-45, 2005

134. Partridge M, Gaballah K, Huang X. Molecular markers for diagnosis and prognosis. Cancer Metastasis Rev 24(1):71-85, 2005

135. Moving AHEAD with an international human epigenome project. Nature 454(7205): 711-5, 2008 
42 


\section{Chapter}

Computer assisted (epi-)genotypic categorization of colorectal cancer identifies

CIMP and TP53 mutations as leading

classification tools

AHG Cleven

S Derks

KM Smits

A Spiertz

$M$ van Engeland

AP de Bruïne

In preparation 
$44 \mid$ Chapter 3

\section{Abstract}

The increasing knowledge of (epi)genetic alterations in CRC and the observed variation in the course of the disease within the same CRC stage, have led to the recognition that classifying CRCs on the basis of molecular events could improve traditional classification. In the current study we molecularly classified CRCs using computer assisted unsupervised hierarchical clustering of genetic and epigenetic alterations and correlated this with clinicopathological parameters.

Genetic-(mutations in APC, KRAS, TP53, BRAF, and microsatellite instability), and epigenetic ( $C p G$ island methylator phenotype, CIMP) alterations were determined in 160 colorectal cancers. Morphologic features were refined by inventarisation of differentiation grade, mucinous differentiation, dirty necrosis, circumscribed tumour growth, tumour budding and lymphocyte infiltration. Unsupervised hierarchical clustering was used to cluster CRCs based on these molecular characteristics. Correlations between these clusters, morphology and outcome were investigated.

Unsupervised hierarchical clustering divided CRC's in CIMP+ (CL2, 22\% (36/160)) and CIMPtumors. The latter group could be further subdivided in TP53 mutated tumors (CL3, 37\% (59/160)), and a group of CIMP- and TP53 wildtype tumors (CL1, 41\% (65/160)). CL2 consisted of two subgroups of $29 \%$ (10/35) microsatellite instable (MSI) and 71\% (25/35) microsatellite stable (MSS) tumors. There was no consistent relationship between these clusters and morphology, except for mucinous differentiation, which was related to right-sided CL2/MSI/TP53 wildtype tumors $(p=0.023)$. There was no impact of computerized clustering on survival of CRC patients. In rectum tumors on the other hand, a poorer patient survival was noted for CL2 tumors as compared to CL3 tumors $(p=0.01)$.

Molecular clustering of colorectal adenocarcinomas shows that CIMP status is the principal classifier, and that both CIMP+ and CIMP- tumors are further classified on the basis of TP53 mutational status. Hierarchical clustering is only modestly related to morphology and outcome, which appear to be dependent on additional factors such as microsatellite stability and localization in the intestine. Although current molecular clustering provides knowledge in the underlying biology of $\mathrm{CRC}$, it did not improve traditional classification with respect to prognostic value. 


\section{Introduction}

The increasing knowledge of molecular alterations in CRC and the observed variation in the course of the disease and treatment response within the same CRC stage, have led to the recognition that classifying CRCs on the basis of molecular events could improve traditional classification ${ }^{1-4}$. CRCs are currently classified according to traditional clinical and pathological features ${ }^{2}$. According to the WHO histological typing system, adenocarcinoma is by far the most prevalent diagnosis $(>95 \%)^{5}$. Adenocarcinomas are graded predominantly on the basis of the extent of glandular formation, and are classified as low-grade (encompassing well and moderately differentiated adenocarcinomas) and highgrade (including poorly differentiated adenocarcinomas and undifferentiated carcinomas) $^{5,6}$. The tumor-node-metastasis (TNM) system ${ }^{7}$, as defined by the UICC (International Union against Cancer, together with the American Joint Committee on Cancer), is the most commonly used staging system, and constitutes the most important prognostic factor and adjuvant treatment indicator for patients with colorectal cancer in routine medical practice. However, this traditional mode of classifying CRC through typing, grading and staging does not account for tumor heterogeneity. Each CRC patient has a unique disease that has been caused by distinctive biology $y^{8,9}$. The presence or absence of specific molecular alterations could predict the response to targeted individualised therapy and overall prognosis in contrast to the traditional classification.

The precise incidence and clinical presentation of molecularly defined profiles in $\mathrm{CRC}$ are subject to ongoing research. With respect to this, genetic instability and DNA methylation play a pivotal role. Well characterized forms of genetic instability are chromosomal instability $(\mathrm{CIN})$ and microsatellite instability $(\mathrm{MSI})^{10,11}$. CIN includes genetic events occurring through accumulation of numerical or structural chromosomal abnormalities (aneuploidy) ${ }^{12}$. The CIN pathway is associated with mutations in key genes involved in the adenomacarcinoma sequence of CRC: APC (85\%), KRAS (40\%) and the tumor suppressor gene TP53 $(50 \%)^{12}$. MSI results from failure of the mismatch repair (MMR) system. In cases of sporadic colorectal cancer, MMR dysfunction is strongly associated with bi-allelic DNA promoter methylation of $h M L H 1^{13}$. A third main pathway in the carcinogenesis of CRC is the $\mathrm{CpG}$ island methylator phenotype (CIMP) which refers to a subset of tumors with an exceptionally high frequency of promoter $\mathrm{CpG}$ island methylation of tumor suppressor-and DNA repair genes. CIMP+ tumors represent a distinct group of tumors, including the majority of cases of sporadic colorectal cancer with MSI and tumors with mutations in the BRAF oncogene ${ }^{14}$.

A more sophisticated theoretical categorization of CRC in five distinct molecular subtypes was proposed by $\mathrm{Jass}^{2}$, based on previous publications of 
underlying genetic instability and the presence of promoter $\mathrm{CpG}$ island methylation within CRC. A group of sporadic CRCs was suggested consisting of CIMP-high, MSI-high and BRAF mutated tumors, presumed to originate in serrated polyps and approximately being present in approximately $12 \%$ of all sporadic CRCs. A second group representing approximately $8 \%$ of sporadic CRCs, consisted of CIMP-high, BRAF mutated and MSI-low tumors, also originating from serrated polyps. CRC tumors with CIMP-low status, MSS or MSI-low with a KRAS mutation, originating from either adenomas or serrated polyps formed a third group, with a presumed $20 \%$ overall frequency within sporadic CRCs. A large group of almost $57 \%$ CRCs, named sporadic or FAPassociated CRCs were designated as a fourth group, containing CIMP- and MSS tumors, supposedly being derived from traditional adenomas. The last minor group (3\% frequency) of CRCs, named Lynch Syndrome associated or familial MSI-H tumors, contained CIMP- and MSI-H tumors, also derived from traditional adenomas ${ }^{2}$. More or less characteristic clinicopathological and morphologic features have been attributed to each of these suggested CRCs subgroups ${ }^{2}$.

Studying the association between molecularly defined clusters in CRC and their clinicopathological features is important for the improvement of therapeutic options within CRC. Therefore, in the light of numerous previous efforts that have been undertaken to unravel the complex underlying molecular changes in CRC, we classified CRC by computer assisted unsupervised hierarchical clustering, based upon well described markers in the genesis of CRC including: CIMP, MSI, APC-, KRAS-, TP53-, and BRAF mutation status, in a series of $160 \mathrm{CRC}$ cases. Subsequently, we investigated in which way this classification was related to patient data, including clinicopathological parameters, morphological features (differentiation grade, mucinous differentiation, dirty necrosis, circumscribed tumour growth, tumour budding and lymphocyte infiltration) and biological behavior in terms of patient prognosis.

\section{Materials and methods}

\section{Patient population}

Patients were entered in two multi-center prospective clinical trials between 1979 and 1981 in the Netherlands. One trial was designed to compare patient survival after treatment of colonic cancer by conventional surgery or the notouch isolation technique ${ }^{15}$. The second trial was conducted to compare survival in rectal cancer patients with or without preoperative radiotherapy. In the current study, we included only the patients who did not undergo preoperative radiotherapy. At the time the trial was conducted, only surgical 
removal of the tumors was performed, and adjuvant chemotherapy was not yet standard practice. Hereby a major advantage is achieved since no bias of different adjuvant therapy protocols was introduced. After surgery, tumor tissues and lymph nodes were fixed in buffered formalin, sectioned, and embedded in paraffin. Experienced pathologists documented the histopathological characteristics of the tumors, including tumor stage, differentiation grade, size, (lymph-)angioinvasion, perineural invasion and lymphnode involvement. Tumor stage was defined according to the TNM staging system.

For both trials, follow up took place every 3 months during the first three years and every 6 months between three and five years after initial diagnosis and surgery. Standard protocols were followed, with routine blood counts and chemistry studies (including CEA levels) at each visit and liver ultrasound, chest x-ray and colonoscopy annually, to evaluate both recurrence of disease and disease-related death. After the initial five year follow up period, only the time and cause of death were registered. Follow-up was complete for all patients. In the present study, failure was defined as death due to recurrent disease, excluding postoperative mortality within 30 days and non-disease related death.

For molecular analysis, tumor tissues from 160 patients with primary colorectal cancer were available. The distribution of age, gender, tumor stage, location and type of tumor, frequency of events and mean follow-up time of the patients in this study are representative for the patients in the trial and are provided in Table 3.1.

Table 3.1 Clinicopathological characteristics of CRC series.

\begin{tabular}{lc}
\hline & Total \\
\hline Age & \\
Mean age (SD) & $67.7(11.6)$ \\
Gender & \\
$\quad$ Male & $75 / 160(47 \%)$ \\
Female & $85 / 160(53 \%)$ \\
Tumor location & \\
Right-sided colon & $59 / 160(37 \%)$ \\
Left-sided colon & $45 / 160(28 \%)$ \\
Rectum & $56 / 160(35 \%)$ \\
Tumor Grade & \\
Well/Moderately & $118 / 141(84 \%)$ \\
Poor & $23 / 141(16 \%)$ \\
CRC Stage & \\
I & $3 / 160(2 \%)$ \\
II & $93 / 160(58 \%)$ \\
III & $47 / 160(29 \%)$ \\
IV & $17 / 160(11 \%)$ \\
Event frequency & $58(37 \%)$ \\
Median follow up time & 4,7 years \\
\hline
\end{tabular}

SD: Standard Deviation, ${ }^{* *}$ colorectal cancer specific death 
$48 \mid$ Chapter 3

\section{Genomic DNA Isolation}

Genomic DNA was extracted from CRC tissues using PureGene ${ }^{\mathrm{TM}}$ Genomic DNA Isolation Kit (Gentra Systems) according to the manufacturer's protocol.

\section{Methylation-specific PCR}

Promoter CpG island methylation of the following genes: calcium channel, voltage-dependent, $T$ type, alpha-1G subunit (CACNA1G), insulin-like growth factor 2 (somatomedin A) (IGF2), neurogenin 1 (NEUROG1), runt-related transcription factor 3 (RUNX3) and suppressor of cytokine signaling 1 (SOCS1), was determined using sodium bisulfite modification of genomic DNA using the EZ DNA methylation kit (ZYMO research Co., Orange, CA). Methylation Specific PCR (MSP) was performed as described in detail elsewhere $^{16,17}$. In brief, to facilitate MSP analysis on DNA retrieved from formalin-fixed, paraffin-embedded tissue, DNA was first amplified with flanking PCR primers, that amplify bisulfite modified DNA but do not preferentially amplify methylated or unmethylated DNA. The resulting fragment was used as a template for the MSP-reaction. All PCRs were performed with a control for unmethylated alleles (normal lymphocyte DNA) and a positive control for methylated alleles (Sssl methyltransferase (New England Biolabs) treated normal lymphocyte DNA) and a negative control without DNA. Each PCR reaction was loaded onto a $2 \%$ agarose gel, stained with Gelstar $^{\circledR}$ (Cambrex Bioscience Rockland Inc, USA) and visualized under UV illumination. Primers and PCR conditions are provided in Supplementary Table 3.S1.

\section{Microsatellite instability}

Microsatellite instability (MSI) was determined by a pentaplex PCR, using the mononucleotide MSI markers BAT-26, BAT-25, NR-21, NR-22 and NR-24, as previously described $^{18}$. MSI was defined to be present if $\geq 3$ of 5 markers (BAT26, BAT-25, NR-21, NR-22 and NR-24) showed allelic size variants.

\section{BRAF, KRAS, APC and TP53 mutation analysis}

The common V600E BRAF mutation in exon 15 was analyzed by a seminested PCR and subsequent RFLP analyses as previously described ${ }^{19}$.

For KRAS mutation analysis a flanking 179 bp PCR product was amplified including codons 12 and 13 as described previously ${ }^{20}$.

Since the majority of somatic mutations in $A P C$ occur within the MCR, we amplified the MCR as four overlapping fragments (codons 1286-1520) in a nested PCR strategy. Flank PCR was performed to generate two fragments $A$ and $B$. Fragment $A$ was used as starting material for the amplification of nested fragments $S 1$ and $S 2$, and fragment B was used for nested fragments S3 and 
S4 as previously described ${ }^{21}$. Mutation analyses of TP53 exons 5-8 was performed using a semi-nested PCR approach, (see supplemental Table 3.S2 for primer sequences). CaCo2 (exon 6, codon 204 non-sense mutation) was included as a positive control. Direct sequencing of PCR products was performed using the BigDye ${ }^{\circledR}$ Terminator v1.1 Cycle Sequencing Kit (Applied Biosystems) and analysed on the ABI 3730 DNA Analyzer (Applied Biosystems). Mutation detection was performed using Mutation Surveyor DNA Variant Analysis Software v3.0 (SoftGenetics LLC, USA). Tumors with silent mutations or a common polymorphism were classified as having wild-type TP53.

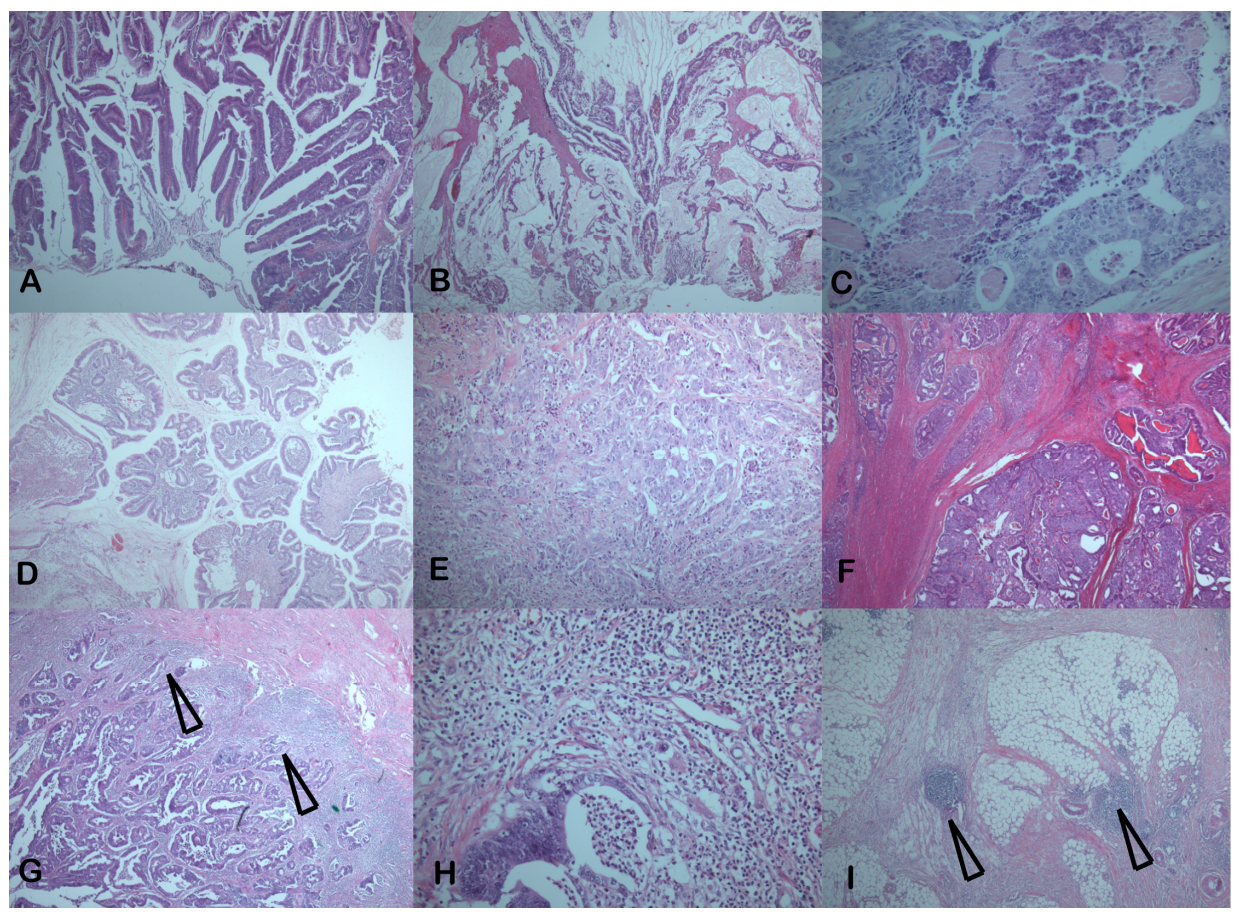

Figure 3.1 Illustrations of CRCs with: (A) serrated morphology; (B) mucinous morphology; (C) locations of "dirty necrosis"; (D) well differentiated tumour morphology; (E) poorly differentiated tumour morphology; $(F)$ circumscribed tumour growth pattern; $(G)$ area of tumour budding (black arrows); $(\mathrm{H})$ tumour infiltrating lymphocytes; (I) localisation of Crohn-like lymphoid aggregates (black arrows). (A,B,D-G, I) Original magnifications $\times$ 20. $(\mathrm{C}, \mathrm{H})$ Original magnifications $\times 40$.

\section{Morphological characteristics}

Figure 3.1 A-I illustrates the morphological characteristics we determined in our study population. Initially we scored several morphological features including: 
$50 \mid$ Chapter 3

serrated morphology, mucinous differentiation, dirty necrosis, differentiation grade, circumscribed tumor growth, tumor budding and lymphocytic infiltration, including Crohn-like host response or diffuse tumor infiltrating lymphocytes. Serrated morphology was left out of our analyses due to the low observed frequency $(n=1)$ in our study-population. Poor differentiation grade was present in $16 \%$ of cases, mucinous differentiation was present in $16 \%$, dirty tumor necrosis in $59 \%$, circumscribed invasion pattern in $35 \%, 21 \%$ showed tumor budding at the invasive margins and $23 \%$ of all cases showed a clear lymphocytic infiltration in the tumor. For details on scoring methods of morphologic characteristics see below.

\section{Differentiation grade}

Tumors were graded by pattern of poorest differentiation, grading was based on the retention of glandular differentiation and given a single grade of differentiation (well, moderately, poor). The worst grade of tumor seen was used for the overall grade. For analysis well and moderately differentiated tumors were grouped together.

\section{Mucinous differentiation}

Tumors with greater than $50 \%$ area showing extracellular mucin were classified as mucinous. Tumors with less than $50 \%$ area showing extracellular mucin were classified as having focal mucinous differentiation. Tumours with no extracellular mucin were classified as negative for mucinous differentiation.

\section{Tumor necrosis}

Tumors were assessed for the presence or absence of "dirty necrosis" defined by intra-tumoral necrotic debris in tumor glands as well as tumor necrosis, often considered a characteristic of colorectal carcinomas. If only a rare focus of necrosis was present $(<10 \%)$ then the tumor was considered negative.

\section{Tumor budding}

When there was a diffuse growth pattern with a transition from glandular structures to single cells or clusters of up to four cells at the invasive margin, tumor budding was present ${ }^{22}$.

\section{Circumscribed}

Tumors with a so-called "pushing" invasive margin were classified as well circumscribed. When the tumor invaded in a diffuse manner with widespread penetration of normal tissues, tumors were named non-circumscribed ${ }^{23}$. 
(Epi-)genotypic categorization of colorectal cancer $\mid 51$

\section{Lymphocytic infiltration}

If peritumoral chronic inflammation was present with or without Crohn's like lymphocytic nodules or if tumor infiltrating lymphocytes were present in the tumor epithelium, lymphocytic infiltration was scored positive ${ }^{6}$.

\section{Data analysis}

CIMP was determined using the panel as suggested by Weisenberger et al. ${ }^{14}$ CRCs are defined as CIMP+ when $>3 / 5$ analyzed markers (CACNA1G, IGF2, NEUROG1, RUNX3, SOCS1) are methylated.

Unsupervised clustering (using Spotfire DecisionSite ${ }^{\circledR}$ for Functional Genomics), based on the similarity of CIMP, MSI, BRAF, APC, KRAS, TP53 status was performed by using half square Euclidian distance (Wards method linkage rule $)^{24,25}$. Correlations between our computed clusters, morphology data and clinicopathological parameters were determined by the Pearson ChiSquare and Fisher's exact test as appropriate. To evaluate the relationship between the calculated clusters and patient survival, Kaplan-Meier survival curves were calculated. The endpoint for analyses was overall survival starting from the day of surgery. Independent variables predicting survival were evaluated by regression analyses using Cox Regression. The Cox-regression model included the variables: CL1, CL2, CL3, age, gender, tumor location, differentiation grade and Stage. All $p$-values are two sided and $p$-values $<0.05$ were considered statistically significant. SPSS 12.0.1 software was used for data analyses.

\section{Results}

\section{CIMP, MSI, BRAF-, APC-, KRAS- and TP53 mutation status and association with tumor location}

A summary of the baseline (epi-)genetic aberrations determined in our study population is provided in Table 3.2. Using the Weisenberger criteria to define CIMP, 25\% (39/156) of CRCs could be classified as CIMP+. This frequency is in line with previous publications were the reported frequency of CIMP+ ranged between $18 \%$ and $25 \%^{14,26-29}$. MSI was detected in $12 \%(19 / 158)$ of our study population, which is in accordance with previous reports ${ }^{30}$. CIMP+ tumors were positively correlated with MSI status, $\mathrm{p}<0.001$ (data not shown). BRAF mutations were detected in $9 \%(14 / 157)$ of cases, which is in line with the reported frequency within CRC by others ${ }^{31,32}$. CIMP as well as MSI were positively correlated with BRAF mutations $(p=0.027$ and $p=0.012$, data not shown), which is in agreement with current understanding of CIMP+ CRC. 
$52 \mid$ Chapter 3

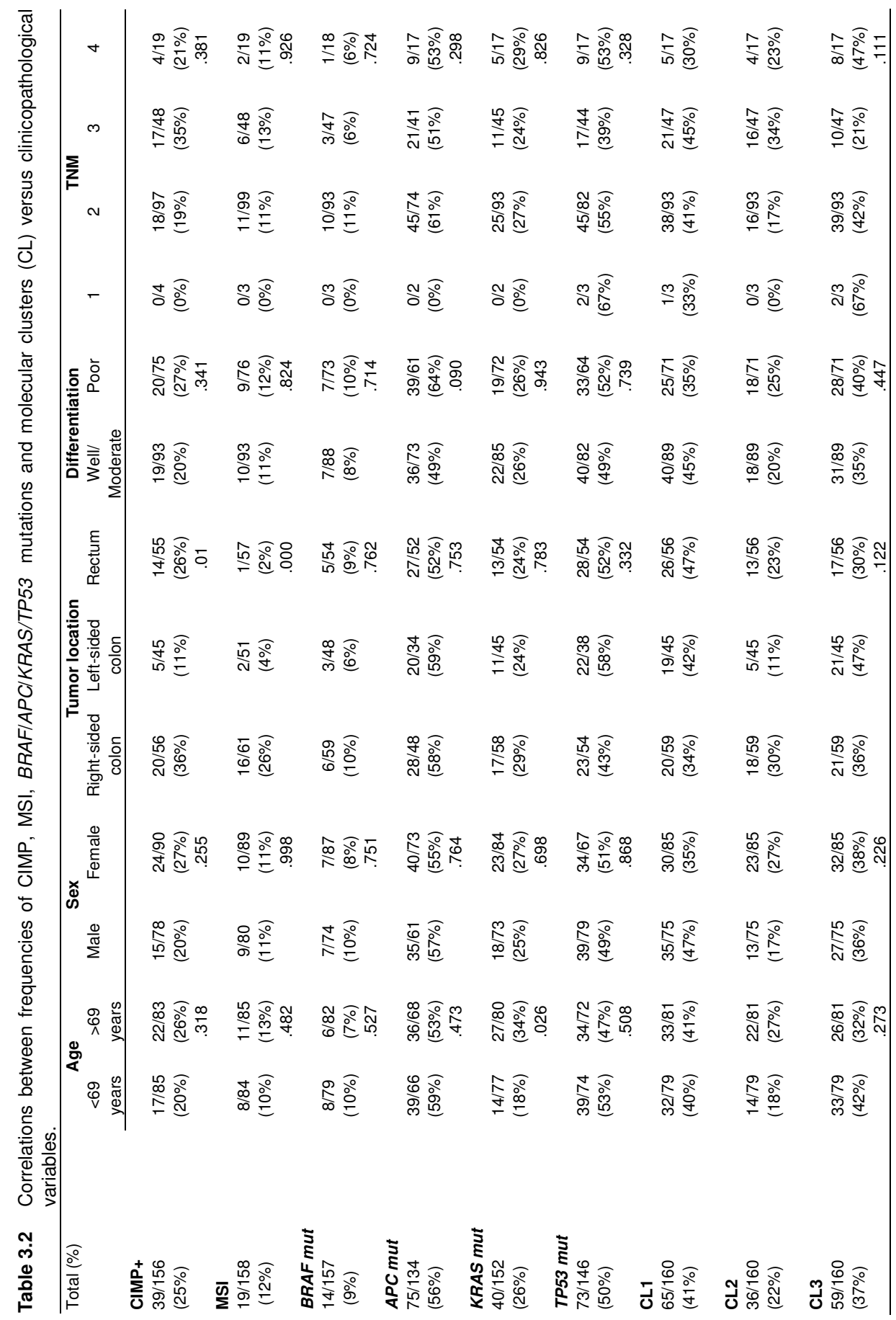


$50 \%(73 / 134)$ of the tumors had a mutation of the $A P C$ gene in the mutation cluster of exon 15. Point mutations in exon 1 of the KRAS gene were found in $26 \%(40 / 152)$ of all cases: $20 \%(30 / 152)$ in codon 12 and $6 \%(10 / 152)$ in codon 13 , respectively. Mutations in exons 5 to 8 of the TP53 gene were present in $53 \%(77 / 146)$ of all cases. TP53 mutations were positively correlated with APC mutations, $\mathrm{p}=0.021$ (data not shown) and not with MSI, CIMP or BRAF mutations, likely reflecting tumors within the CIN category. The frequency of $A P C, K R A S$ and TP53 mutations in our study population are in accordance with the frequencies reported by others in $\mathrm{CRC}^{3,31-33}$.

Regarding baseline clinicopathological features, CIMP+ tumors were more often located in the right sided colon (located proximal to the splenic flexure) $36 \%(20 / 56)$, and rectal tumors $26 \%(14 / 55)$, compared to left sided colon tumors (located distal to the splenic flexure) 11\% (5/45), $p=0.01$ (Table 3.2). Furthermore MSI was positively correlated with right sided tumor location, $26 \%$ $(16 / 61)$ of right-sided colon tumors were MSI tumors, compared to $4 \%(2 / 51)$ in the left sided colon and $2 \%(1 / 57)$ in the rectum $(p<0.001)$. BRAF-, APC-, KRAS- and TP53 mutations did not correlate with tumor location (Table 3.2).

\section{Molecular clusters and associations with clinicopathological characteristics}

Unsupervised hierarchical clustering, based on (epi)genetic changes including CIMP, MSI, BRAF-, APC-, KRAS, and TP53 mutations, resulted in three main clusters (CL1, CL2 and CL3, Figure 3.2, Table 3.2), which were reproducible with a similar linkage distance, independent of the sequence of input variables and stage of disease (data not shown). CL2 was characterized by CIMP+, and accounted for $22 \%(36 / 160)$ of CRC's. The remaining $78 \%$ of CIMP- tumors were subdivided into TP53 mutated tumors in CL3, comprising 37\% (59/160) of cases, and a remaining subset of TP53 wildtype tumors representing CL1, containing $41 \%(65 / 160)$ of cases, respectively $p<0.001$ and $p<0.001$ (Figure 3.3A). Overall, MSI was more prevalent in CL2 tumors $29 \%$ (10/35), than in CL1 6\% (4/64) or CL3 9\% (5/59), $\mathrm{p}<0.01$ (Figure 3.3A). CL3 tumors more often harbored $A P C$ mutations $(71 \%, 35 / 49)$, as compared to CL1 $(45 \%, 23 / 51)$ and CL2 $(50 \%, 17 / 34), p=0.021$, (Figure $3.3 A)$. To the contrary, BRAF and KRAS mutations were more or less equally distributed among the three clusters and thus did not give rise to separate clusters (Figure 3.3A). Furthermore, there was no consistent relationship between computerized clustering, morphology and other clinicopathological variables (Figure 3.3B, Table 3.2). Figure 3.3B illustrates the distribution of mucinous differentiation, dirty necrosis, differentiation grade, circumscribed tumor growth, tumor budding and lymphocyte infiltration among the three (epi)-genetic clusters. 


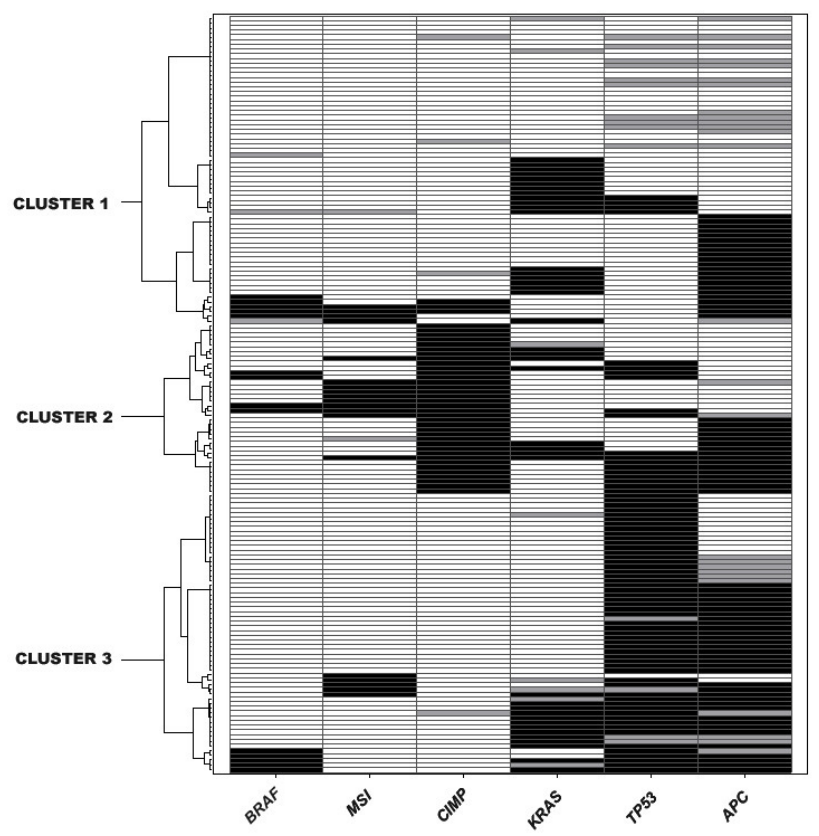

Figure 3.2 CRCs clusters CL1 (41\%, 65/160), CL2 (22\%, 36/160), CL3 (37\%, 59/160) obtained by unsupervised hierarchical clustering of CIMP, MSI, BRAF/KRAS/APC and TP53 mutation status. (Black box indicates positive, white box indicates negative, grey box indicates missing value).

Interestingly, CIMP+ tumors of CL2 could be subcategorized on the basis of MSI status and TP53 mutation status as follows. CL2 consisted of 29\% (10/35) being $\mathrm{MSI}$, a larger group of $71 \%(25 / 35)$ being MSS. Within CL2, MSI tumors more often were localized in the right hemicolon $90 \%(9 / 10)$ than in the rectum $10 \%(1 / 10)$ or left-sided colon $0 \%, p<0.01$ (Figure $3.3 \mathrm{C}$ ). There was no consistent relationship between computerized clustering and morphology, except for right-sided MSI tumors in CL2 which more often showed mucinous differentiation $44 \%(4 / 9)$, compared to $0 \%(0 / 9)$ in rectum tumors, $p=0.023$ (data not shown). Although not significant $20 \%$ (3/15) of TP53 mutated tumors were MSI and $80 \%(12 / 15)$ MSS, $p=0.46$ (data not shown). Furthermore, within CL2 tumors, TP53 status seems to be related with tumor localization in the bowel: $33 \%(5 / 15)$ of TP53 mutated cases were right-sided colon tumors compared to $54 \%(8 / 15)$ of TP53 mutated cases being rectum tumors (borderline significance $p=0.06$, Figure $3.3 \mathrm{C}$ ). 

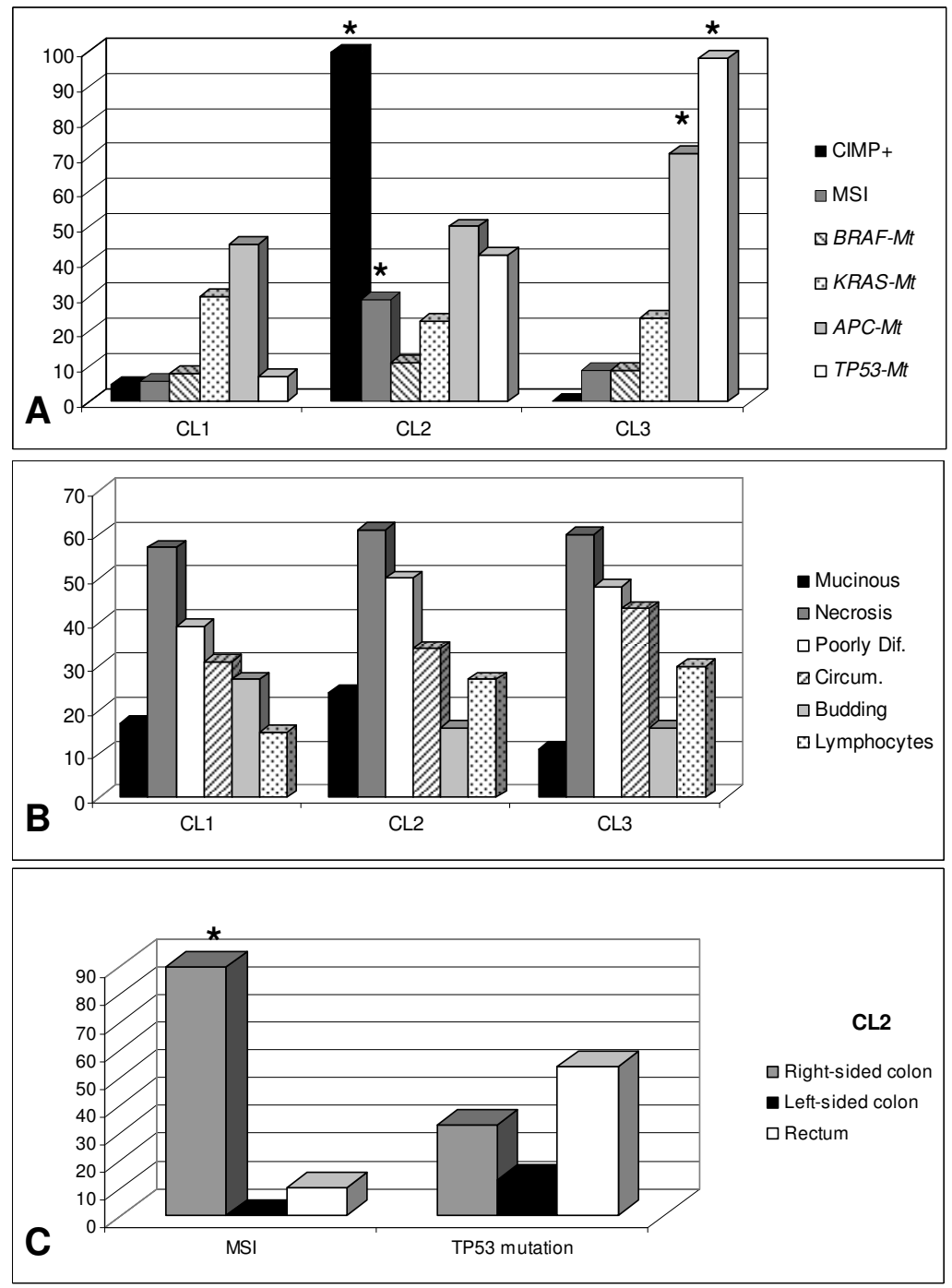

Figure 3.3 (A) Percentages of CIMP+, MSI, BRAF/KRAS/APC/TP53 mutated tumors within the three clusters CL1, CL2 and CL3 (bars indicate percentage of positive cases within each cluster).

(B) Percentages of positive scoring of morphology markers: differentiation grade, mucinous differentiation, dirty necrosis, circumscribed tumour growth, tumour budding and lymphocyte infiltration within CL1, CL2 and CL3 (bars indicate percentage of positive cases within each cluster).

(C) Within CL2 tumors distribution of MSI and TP53 mutations, according to site of origin, respectively right-sided colon, left-sided colon and rectum (bars indicate percentage of positive cases).

* indicates significant $p$-values 
$56 \mid$ Chapter 3

CIMP- tumors were further subdivided depending on TP53 mutational status, into CL1 TP53 wildtype tumors and CL3 TP53 mutated tumors respectively. Overall, within CL3, TP53 mutated tumors were equally distributed among right-sided colon 32\% (17/54), left-sided colon 37\% (20/54) and rectum 32\% (17/54) (data not shown). Within CL3 we could not identify distinct patterns of morphology between (epi)genetic aberrations. As opposed to CL3 and CL2, CL1 represented a remaining group (41\%), of CIMP- and TP53 wild type tumors, which were not characterized by a single main (epi)-genetic aberration, as illustrated in Figure 3.3A. Within CL1, APC mutations (45\%) and KRAS mutations (30\%) were frequently present, in contrast to MSI, CIMP+ and TP53 mutations, which showed an overall low frequency $(<10 \%)$. Similar to cluster CL3, within CL1 we could not identify differences between (epi)-genetic aberrations and clinicopathological variables.

Subsequently we verified whether a particular cluster did relate to patient survival. Overall and after stratification for tumor stage, there was no impact of computerized clustering on survival in colon tumors (Figure 3.4A-D), $p=0.24$, $p=0.35, p=0.45, p=0.39$, respectively. Furthermore if we stratified for tumor location, no survival differences between the clusters were observed within proximal and distal colon tumors (Figure 3.4E, $F, p=0.82$ and $p=0.98$, respectively). In rectal tumors $(n=56)$ on the other hand, a poorer prognosis was noted for CL2 tumors as compared to CL3 and CL1 tumors (Figure 3.4G, $\mathrm{p}=0.01)$. CL2 tumors, being $\mathrm{CIMP}_{+}$, showed lower patient survival rates compared to CL3 lesions, containing TP53 mutations. This observation could not be confirmed in a multivariate Cox regression model (Table 3.3). Although CL2 tumors showed a HR of 2.35 (Cl: 0.77-8.32), no statistical significance was reached, $p=0.13$. Probably the effect of $C L 2$ is overruled by tumor stage IV, showing a $\mathrm{HR}=12.62(\mathrm{Cl}: 2.75-58.0), \mathrm{p}<0.001$.

Table 3.3 Cox proportional hazard model in rectum tumors $(n=49)$.

\begin{tabular}{lcc}
\hline & $\begin{array}{c}\text { Event: CRC mortality } \\
\text { HR }(95 \% \mathrm{Cl})\end{array}$ & p-value \\
\hline $\begin{array}{l}\text { Clusters } \\
\text { CL2* }\end{array}$ & $2.53(0.77-8.31)$ & 0.13 \\
CL3 $^{*}$ & $0.79(0.20-3.16)$ & 0.74 \\
Age (years) & $1.03(0.98-1.09)$ & 0.26 \\
Gender (female & $1.59(0.50-4.98)$ & 0.43 \\
Differentiation grade (poor $\left.{ }^{*}\right)$ & $1.92(0.54-6.78)$ & 0.31 \\
Stage & & 0.78 \\
III & & \\
IV & $1.17(0.38-3.63)$ & $<0.001$ \\
\hline
\end{tabular}

HR: Hazard Ratio=Relative Risk. *Reference group = CL1; " Reference group = Man, "Reference group $=$ Well $/$ moderate, "Reference group $=$ Stage II . 

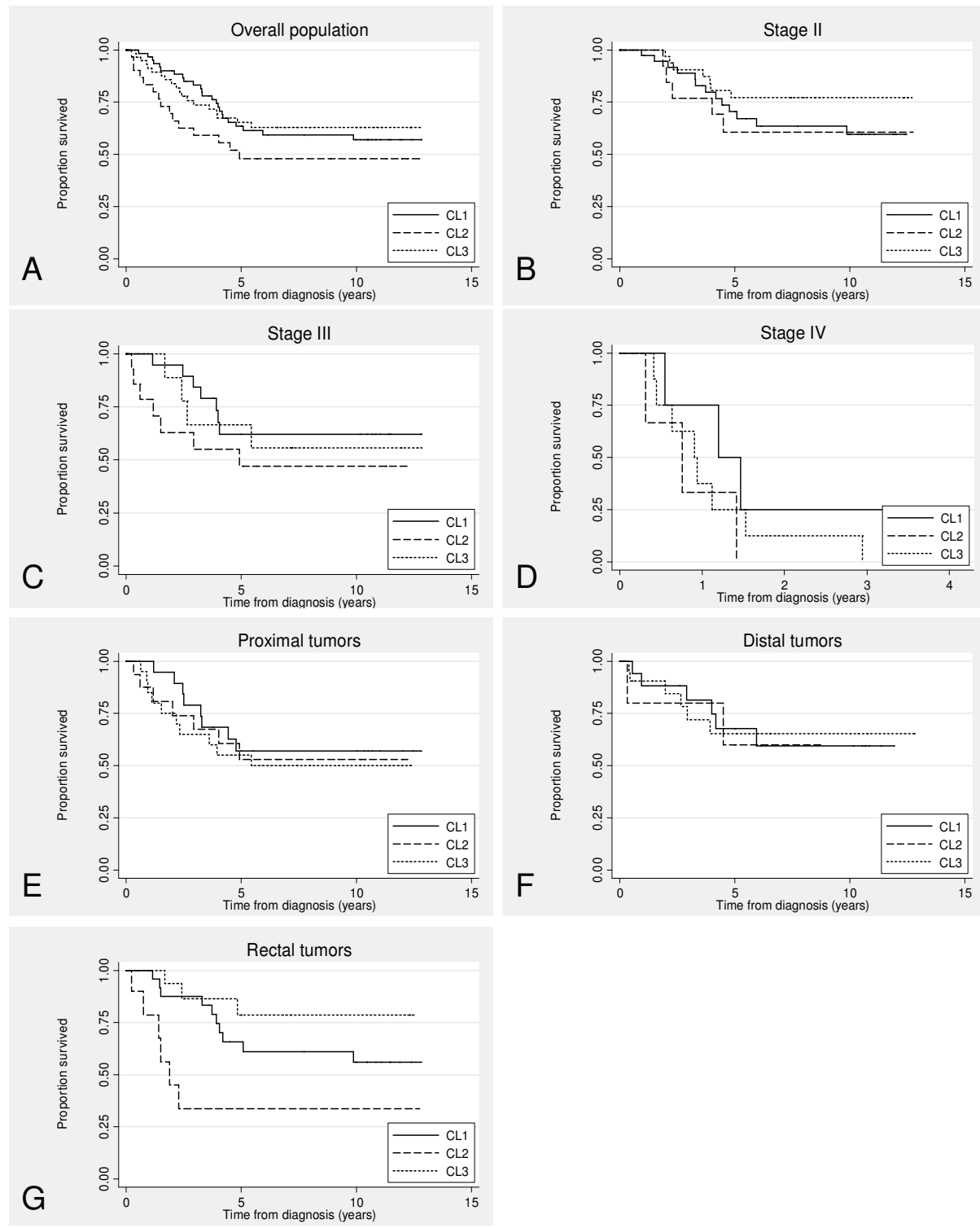

Figure 3.4 (A) Survival curves overall population of CL1 $(n=65)$ versus CL2 $(n=36)$ and CL3 $(n=59), p=0.24$; (B) Survival curves within Stage II of CL1 $(n=38)$ versus CL2 $(n=16)$ and CL3 ( $n=39), p=0.35$; (C) Survival curves within Stage III of CL1 $(n=21)$ versus CL2 $(n=16)$ and CL3 $(n=9), p=0.45$; (D) Survival curves within Stage IV of CL1 $(n=5)$ versus CL2 $(n=4)$ and CL3 $(n=8), p=0.39$; (E) Survival curves within proximal colon tumors of CL1 $(n=20)$ versus CL2 $(n=18)$ and CL3 $(n=21), p=0.82$; $(F)$ Survival curves within distal colon tumors of CL1 $(n=19)$ versus CL2 $(n=5)$ and CL3 $(n=21), p=0.98 ;(G)$ Survival curves within rectal tumors of CL1 $(n=26)$ versus CL2 $(n=13)$ and CL3 $(n=17)$, $\mathrm{p}=0.012$. 
$58 \mid$ Chapter 3

\section{Discussion}

Studying the association between molecular clusters in CRC and their clinicopathological features may lead tot a better understanding of the disease. Therefore, in the light of numerous previous efforts that have been undertaken to unravel the complex underlying molecular changes in $\mathrm{CRC}$, we molecularly classified CRC using computer assisted unsupervised hierarchical clustering of genetic and epigenetic alterations and correlated this with clinicopathological parameters.

Computer assisted clustering divided CRC's in CIMP+ $(22 \%)$ and CIMP- (78\%) tumors. The latter group could be further subdivided in TP53 mutated tumors (37\%), and TP53 wildtype tumors (41\%). The cluster of CIMP+ tumors consisted of two subgroups: $29 \%$ microsatellite instable (MSI) and $71 \%$ microsatellite stable (MSS) tumors. A subdivision of CIMP+ tumors, has already been suggested by Jass et al. and others: CIMP1 (MSI associated) and CIMP2 (non-MSI associated) 2,34,35. Recently it was suggested that in CRC, DNA methylation epigenotypes do exist, a high-methylation epigenotype which significantly correlates with $\mathrm{MSI}$, right-sided tumor location and a high frequency of mucinous differentiation, and an intermediate-epigenotype which significantly correlates with distal tumor location, high TP53 expression and a low frequency of mucinous differentiation ${ }^{36,37}$. This subdivision is in concordance with $\mathrm{CIMP}_{+}$, right-sided colon tumors in our population, being MSI and more often displaying mucinous differentiation, and CIMP+ tumors in the rectum, which were more often TP53 mutated, compared to those located in the right-sided colon. It has been shown that right-sided colon and distal colorectal cancers show distinct gene-specific methylation profiles and clinical and molecular characteristics ${ }^{38}$. Although there is scarce literature specifically on the subject of (epi)-genetic profiles in the rectum compared to those in the right-sided colon, it seems that CIMP exists in both MSI, right-sided tumors and MSS distal (rectum) tumors. CIMP has been postulated to explain silencing of the MLH1 gene in right-sided CRC with MSI-High ${ }^{39}$. The precise role or target genes/-pathways of CIMP in distal MSS tumors is unclear. Probably distal CIMP+ tumors originate via different pathways compared to proximal CIMP+ tumors. As already stated, CIMP+ tumors in the rectum seem to originate in a background of CIN since they more often contain TP53 mutations and are MSS $^{36}$. Recently it was shown that $71 \%$ of MSS tumors with CIMP+ status have $A P C$ mutations, suggesting an interrelation with the CIN pathway ${ }^{40}$.

Our study suggests that TP53 is the second most important classifier for CRCs after CIMP. Within CL3, 98\% of tumors harboured a TP53 mutation $(p<0.001)$, and $71 \%$ an $A P C$ mutation $(p=0.021)$ all being CIMP-. Sweeney et al showed in 917 CRCs, that TP53 and APC mutated tumors formed a distinct cluster within CIMP- $\mathrm{CRCs}^{40}$. Jass et al., also suggested the existence of a distinct CRC 
group consisting of CIMP-/MSS, APC/TP53 mutated tumors, which overlaps with CL3 tumors in our study population. Moreover, Jass et all suggested that these tumors have characteristic morphological features, namely dirty tumor necrosis and tumor budding. We did not find a correlation with any specific morphological feature, and in line with our findings the impact of $A P C$ and TP53 mutations on clinical outcome has been shown to be far from conclusive in other studies ${ }^{41,42}$.

Overall there was a poor relation between our molecular clusters and morphological features. Only mucinous differentiation was associated with a subgroup of CIMP+, MSI, right-sided tumors. On the other hand, dirty necrosis, circumscribed tumor growth, tumor budding and lymphocyte infiltration did not show a relation with our molecular clusters. Several explanations are possible for this poor correlation. In our population, $43 \%$ of well differentiated tumors showed mucinous differentiation compared to $15 \%$ in moderately and $12 \%$ in poorly differentiated tumors ( $\mathrm{p}=0.016$, data not shown). Furthermore, none of well differentiated tumors showed tumor budding compared to $22 \%$ in moderately and $24 \%$ in poorly differentiated tumors ( $p=0.13$, data not shown). These results indicate that some morphological parameters are tightly associated with the differentiation grade of a tumor. The poor correlation between our molecularly defined clusters and morphological features (other than mucinous differentiation) can be explained in part by differentiation grade: in a tumor with a higher level of differentiation, probably a single dominant gene is still able to dictate its morphology. To the contrary, in moderately and poorly differentiated tumors, a cumulative increase in epi-genetic aberrations makes it difficult for a single gene to dominate and dictate tumor morphology. Probably this explains why mucinous differentiation, strongly coupled to a better differentiation grade, can be related to a single genetic aberration like MSI. Along similar lines, this explains why morphological features as tumor budding, circumscribed tumor growth and dirty tumor necrosis, which are closely connected to a poorer differentiation grade, are not related to single epi-genetic aberrations.

We could not demonstrate an impact of computerized clustering on survival of CRC patients. Only in rectum tumors, a poorer patient survival was noted for CL2 tumors as compared to CL3 tumors. This was observed in a relatively small number of rectal tumors and could therefore not be confirmed in multivariate analysis taking into account other established prognostic factors such as tumor localization, age, gender and tumor stage. Few studies have evaluated $\mathrm{CIMP}+$ as a prognostic marker in distal tumors, in particular rectal cancer $^{43}$. De Maat and colleagues reported the quantitative MINT locus methylation profile (=surrogate marker for CIMP) and its association with local recurrence in rectal cancers ${ }^{44}$. Based on the methylation profile of MINT3 and 
$60 \mid$ Chapter 3

MINT17 they were able to subdivide patients in to groups with an increased risk for local recurrence.

Although current molecular clustering provides knowledge in the underlying biology of CRC and partly confirms the molecular classification proposed by Jass et al. and others, our computed molecular clusters did not have strong clinicopathological correlations, and therefore could not improve traditional classification with respect to prognostic power. Maybe this was due to the relatively small population size, or the fact that we included a lot of single genetic aberrations compared to a few epigenetic aberrations. The overall frequency of genetic aberrations like mutations in APC, KRAS and TP53 in CRC is relatively low, and additional mechanism such as epigenetic aberrations contribute to tumor progression and prognosis ${ }^{45}$. Another explanation for the lack of prognostic power for our molecular clusters, could be the fact that we did not include chromosomal aberrations, which are associated with a poorer patient survival in $\mathrm{CRC}^{46,47}$. Significantly, recent advances in technology enable researchers to determine the genetic and epigenetic alterations in cancers on a genome-wide scale ${ }^{9,48-50}$. It reveals true biomarkers that enable monitoring of individual tumor progression, tailoring response to therapeutic treatment, and identification of residual disease at a level previously undetectable by current methods ${ }^{51,52}$. Future research should point out whether these genome wide screening methods are able to identify new molecular markers that improve traditional classification.

In summary, computer assisted classification of CRCs shows that CIMP status is the principal classifier, and that both CIMP+ and CIMP- tumors are further classified on the basis of TP53 mutational status. Hierarchical molecular clustering is only modestly related to morphology and outcome. However, there was evidence that some clusters represent biologically distinct subgroups, which appear to be dependent on additional factors such as microsatellite stability and localization in the intestine. CIMP+ CRC appears to comprise two subsets; one localized in the right-sided colon, with $\mathrm{MSI}$ and mucinous differentiation, the other occurring in the rectum, being MSS. 
(Epi-)genotypic categorization of colorectal cancer $\mid 61$

\section{References}

1. Shen L, Toyota M, Kondo $\mathrm{Y}$, et al: Integrated genetic and epigenetic analysis identifies three different subclasses of colon cancer. Proc Natl Acad Sci U S A 104:18654-9, 2007

2. Jass JR: Classification of colorectal cancer based on correlation of clinical, morphological and molecular features. Histopathology 50:113-30, 2007

3. Smith G, Carey FA, Beattie J, et al: Mutations in APC, Kirsten-ras, and p53--alternative genetic pathways to colorectal cancer. Proc Natl Acad Sci U S A 99:9433-8, 2002

4. Vogelstein B, Bearon ER, Hamilton SR: Genetic alterations during colorectal-tumor development. New England Journal of Medicine 319:525-32, 1988

5. Hamilton SR, A.Aaltonen L: Pathology \& genetics of Tumours of the Digestive System, in Kleihues P, Sobin LH (eds): World Health Organization of the Digestive System. Lyon, France, International Agency for Research on Cancer, 2000

6. Jass JR, Do KA, Simms LA, et al: Morphology of sporadic colorectal cancer with DNA replication errors. Gut 42:673-9, 1998

7. Compton CC, Greene FL: The staging of colorectal cancer: 2004 and beyond. CA Cancer J Clin 54:295-308, 2004

8. Jass JR: Molecular heterogeneity of colorectal cancer: Implications for cancer control. Surg Oncol 16 Suppl 1:S7-9, 2007

9. Wood LD, Parsons DW, Jones S, et al: The genomic landscapes of human breast and colorectal cancers. Science 318:1108-13, 2007

10. Gologan A, Sepulveda AR: Microsatellite instability and DNA mismatch repair deficiency testing in hereditary and sporadic gastrointestinal cancers. Clin Lab Med 25:179-96, 2005

11. Issa JP: Colon cancer: it's CIN or CIMP. Clin Cancer Res 14:5939-40, 2008

12. Ogino S, Goel A: Molecular Classification and Correlates in Colorectal Cancer. J Mol Diagn 10:13-27, 2008

13. Worthley DL, Whitehall VL, Spring KJ, et al: Colorectal carcinogenesis: road maps to cancer. World J Gastroenterol 13:3784-91, 2007

14. Weisenberger DJ, Siegmund KD, Campan M, et al: CpG island methylator phenotype underlies sporadic microsatellite instability and is tightly associated with BRAF mutation in colorectal cancer. Nat Genet 38:787-93, 2006

15. Wiggers $\mathrm{T}$, Jeekel $\mathrm{J}$, Arends $\mathrm{JW}$, et al: No-touch isolation technique in colon cancer: a controlled prospective trial. Br J Surg 75:409-15, 1988

16. Herman JG, Graff JR, Myohanen S, et al: Methylation-specific PCR: a novel PCR assay for methylation status of CpG islands. Proc Natl Acad Sci U S A 93:9821-6, 1996

17. Derks S, Lentjes MH, Hellebrekers DM, et al: Methylation-specific PCR unraveled. Cell Oncol 26:291-9, 2004

18. Suraweera N, Duval A, Reperant M, et al: Evaluation of tumor microsatellite instability using five quasimonomorphic mononucleotide repeats and pentaplex PCR. Gastroenterology 123:1804-11, 2002

19. Sieben NL, Roemen GM, Oosting J, et al: Clonal analysis favours a monoclonal origin for serous borderline tumours with peritoneal implants. J Pathol 210:405-11, 2006

20. Sieben NL, Macropoulos P, Roemen GM, et al: In ovarian neoplasms, BRAF, but not KRAS, mutations are restricted to low-grade serous tumours. J Pathol 202:336-40, 2004

21. Luchtenborg M, Weijenberg MP, Wark PA, et al: Mutations in APC, CTNNB1 and K-ras genes and expression of hMLH1 in sporadic colorectal carcinomas from the Netherlands Cohort Study. BMC Cancer 5:160, 2005

22. Shinto $\mathrm{E}$, Mochizuki $\mathrm{H}$, Ueno $\mathrm{H}$, et al: A novel classification of tumour budding in colorectal cancer based on the presence of cytoplasmic pseudo-fragments around budding foci. Histopathology 47:25-31, 2005

23. Jass JR, Love SB, Northover JM: A new prognostic classification of rectal cancer. Lancet 1:1303-6, 1987

24. Carlis J, Bruso K: How many clusters to report: A recursive heuristic. Conf Proc IEEE Eng Med Biol Soc 1:1069-72, 2010 
$62 \mid$ Chapter 3

25. Kaushal D, Naeve CW: Loading and preparing data for analysis in spotfire. Curr Protoc Bioinformatics Chapter 7:Unit 7 8, 2004

26. Kawasaki T, Ohnishi M, Nosho K, et al: $\mathrm{CpG}$ island methylator phenotype-low (CIMP-low) colorectal cancer shows not only few methylated CIMP-high-specific CpG islands, but also low-level methylation at individual loci. Mod Pathol, 2008

27. Lee S, Cho NY, Choi M, et al: Clinicopathological features of CpG island methylator phenotype-positive colorectal cancer and its adverse prognosis in relation to KRAS/BRAF mutation. Pathol Int 58:104-13, 2008

28. Ogino S, Cantor M, Kawasaki T, et al: $\mathrm{CpG}$ island methylator phenotype (CIMP) of colorectal cancer is best characterised by quantitative DNA methylation analysis and prospective cohort studies. Gut 55:1000-6, 2006

29. Hughes LA, Khalid-de Bakker CA, Smits KM, et al: The CpG island methylator phenotype in colorectal cancer: Progress and problems. Biochim Biophys Acta 1825:77-85, 2011

30. Grady WM: Genomic instability and colon cancer. Cancer Metastasis Rev 23:11-27, 2004

31. Hutchins G, Southward K, Handley K, et al: Value of mismatch repair, KRAS, and BRAF mutations in predicting recurrence and benefits from chemotherapy in colorectal cancer. $J$ Clin Oncol 29:1261-70, 2011

32. Nagasaka T, Koi M, Kloor M, et al: Mutations in both KRAS and BRAF may contribute to the methylator phenotype in colon cancer. Gastroenterology 134:1950-60, 1960 e1, 2008

33. Leslie A, Pratt NR, Gillespie K, et al: Mutations of APC, K-ras, and p53 are associated with specific chromosomal aberrations in colorectal adenocarcinomas. Cancer Res 63:4656-61, 2003

34. Issa JP, Shen L, Toyota M: CIMP, at last. Gastroenterology 129:1121-4, 2005

35. Sanchez JA, Krumroy L, Plummer S, et al: Genetic and epigenetic classifications define clinical phenotypes and determine patient outcomes in colorectal cancer. $\mathrm{Br} \mathrm{J}$ Surg 96:1196204, 2009

36. Yagi K, Akagi $\mathrm{K}$, Hayashi $\mathrm{H}$, et al: Three DNA methylation epigenotypes in human colorectal cancer. Clin Cancer Res 16:21-33, 2010

37. Tanaka H, Deng G, Matsuzaki K, et al: BRAF mutation, $\mathrm{CpG}$ island methylator phenotype and microsatellite instability occur more frequently and concordantly in mucinous than nonmucinous colorectal cancer. Int J Cancer 118:2765-71, 2006

38. Deng G, Kakar S, Tanaka H, et al: Proximal and distal colorectal cancers show distinct genespecific methylation profiles and clinical and molecular characteristics. Eur J Cancer 44:1290301, 2008

39. Imai K, Yamamoto $\mathrm{H}$ : Carcinogenesis and microsatellite instability: the interrelationship between genetics and epigenetics. Carcinogenesis 29:673-80, 2008

40. Sweeney C, Boucher KM, Samowitz WS, et al: Oncogenetic tree model of somatic mutations and DNA methylation in colon tumors. Genes Chromosomes Cancer 48:1-9, 2009

41. Russo A, Bazan V, lacopetta B, et al: The TP53 colorectal cancer international collaborative study on the prognostic and predictive significance of p53 mutation: influence of tumor site, type of mutation, and adjuvant treatment. J Clin Oncol 23:7518-28, 2005

42. Zlobec I, Lugli A: Prognostic and predictive factors in colorectal cancer: A critical review. J Clin Pathol, 2008

43. de Maat MF, Narita N, Benard A, et al: Development of sporadic microsatellite instability in colorectal tumors involves hypermethylation at methylated-in-tumor loci in adenoma. Am J Pathol 177:2347-56, 2010

44. de Maat MF, van de Velde CJ, Benard A, et al: Identification of a quantitative MINT locus methylation profile predicting local regional recurrence of rectal cancer. Clin Cancer Res 16:2811-8, 2010

45. Smits K, Cleven AH, Weijenberg MP, et al: Pharmacoepigenomics in colorectal cancer: a step forward in predicting prognosis and treatment response. Pharmocogenomics 9:1903-16, 2008

46. Walther A, Houlston R, Tomlinson I: Association between chromosomal instability and prognosis in colorectal cancer: a meta-analysis. Gut 57:941-50, 2008 
(Epi-)genotypic categorization of colorectal cancer $\mid 63$

47. Bruin SC, He Y, Mikolajewska-Hanclich I, et al: Molecular alterations associated with liver metastases development in colorectal cancer patients. $\mathrm{Br} J$ Cancer 105:281-7, 2011

48. Kibriya MG, Raza M, Jasmine F, et al: A genome-wide DNA methylation study in colorectal carcinoma. BMC Med Genomics 4:50, 2011

49. Timmermann B, Kerick M, Roehr C, et al: Somatic mutation profiles of MSI and MSS colorectal cancer identified by whole exome next generation sequencing and bioinformatics analysis. PLoS One 5:e15661, 2010

50. Sjoblom T, Jones S, Wood LD, et al: The consensus coding sequences of human breast and colorectal cancers. Science 314:268-74, 2006

51. Leary RJ, Kinde I, Diehl F, et al: Development of personalized tumor biomarkers using massively parallel sequencing. Sci Transl Med 2:20ra14, 2010

52. Wang Q, Chaerkady R, Wu J, et al: Mutant proteins as cancer-specific biomarkers. Proc Natl Acad Sci U S A 108:2444-9, 2011 
$64 \mid$ Chapter 3

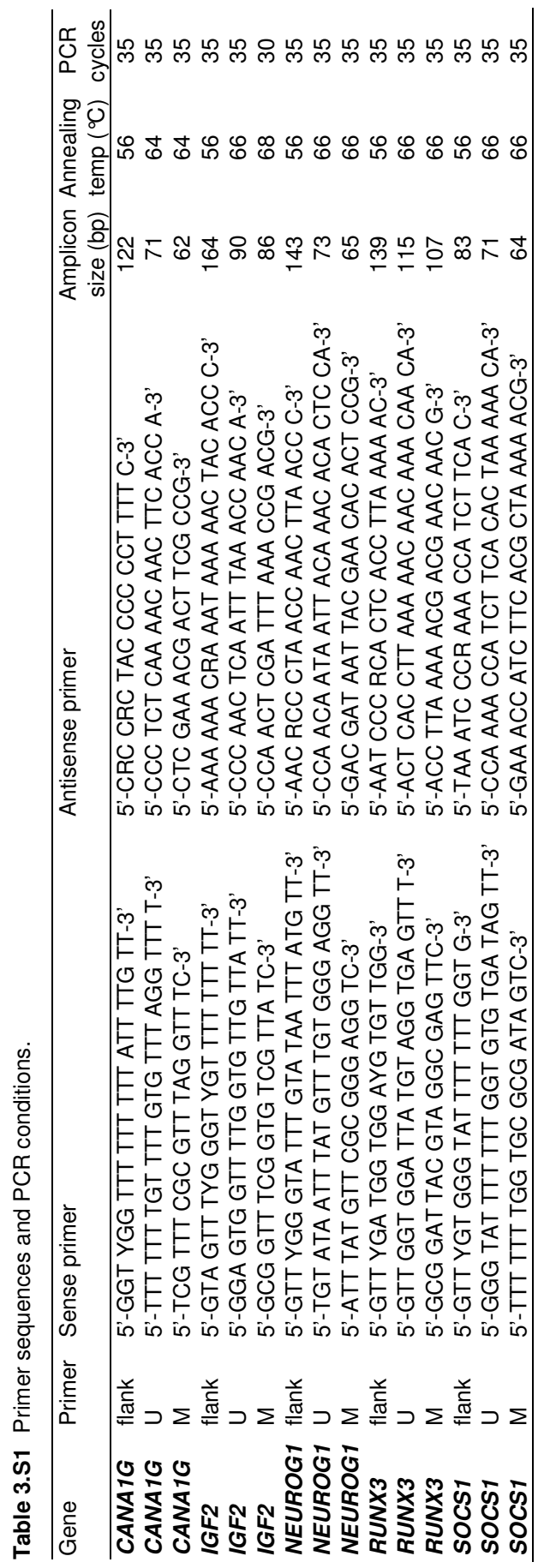


(Epi-)genotypic categorization of colorectal cancer $\mid 65$

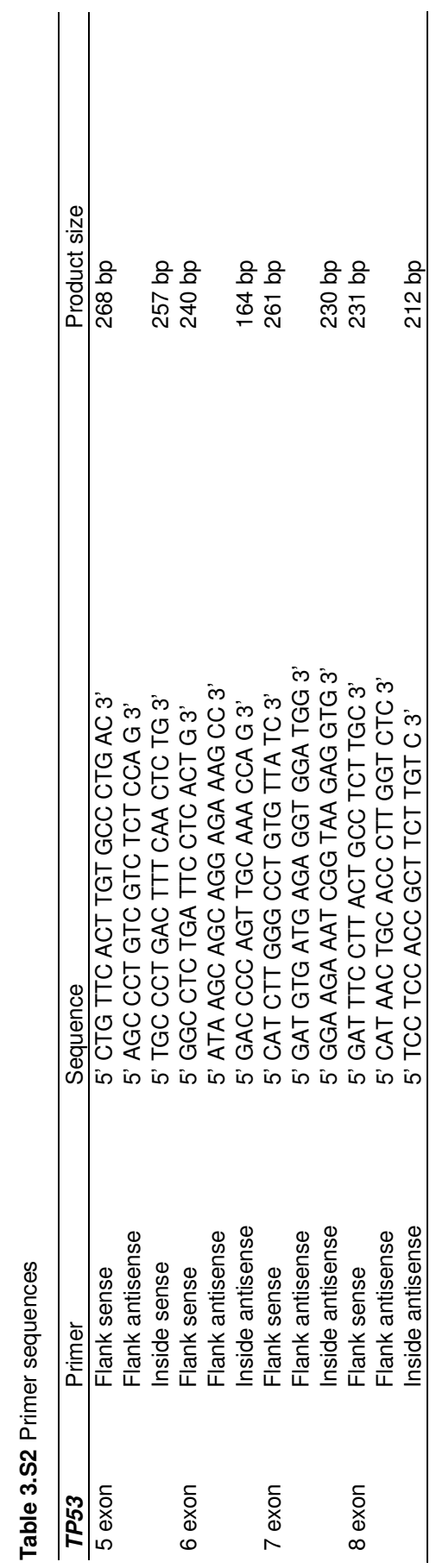


66 


\title{
Chapter 4
}

CHFR promoter CpG island methylation is an indicator of poor prognosis in stage II microsatellite stable colorectal cancer

\author{
AHG Cleven \\ $S$ Derks \\ KM Smits \\ MP Weijenberg \\ JG Herman \\ AP de Bruïne \\ $M$ van Engeland \\ Submitted
}




\section{Abstract}

Data on the prognostic significance of promoter $\mathrm{CpG}$ island methylation in colorectal cancer (CRC) are conflicting, possibly due to associations between methylation and other factors affecting survival such as genetic alterations and use of adjuvant therapy. Here we examine the prognostic impact of promoter methylation in CRC patients treated with surgery alone in the context of microsatellite instability (MSI), BRAF- and KRAS mutations.

173 CRCs were analyzed for promoter methylation of 19 tumor suppressor- and DNA repair genes, the $\mathrm{CpG}$ island methylator phenotype (CIMP), MSI, the exon 15 V600E BRAF mutation and KRAS codon 12 and 13 mutations.

Unsupervised hierarchical clustering based on methylation status of 19 genes revealed three subgroups: cluster 1 (CL1, 57\% (98/173) of CRCs), cluster 2 (CL2, 25\% (43/173) of CRCs) and cluster 3 (CL3, 18\% (32/173) of CRCs). CL3 had the highest methylation index (0.25, 0.49 and 0.69 respectively, $p=<0.01)$ and was strongly associated with CIMP $(p<0.01)$. After stratification for tumor stage, $\mathrm{MSI}$ and $B R A F$ status, no statistically significant differences in survival between CL1, CL2 and CL3 nor between CIMP and non-CIMP CRCs were detected. Analyzing genes separately revealed that $C H F R$ promoter methylation was associated with a poor prognosis in stage II, MSS, $B R A F$ wild-type $C R C s$ : $\mathrm{HR}=3.89(95 \% \mathrm{Cl}=1.58-9.60, \mathrm{p}=0.003)$ and $\mathrm{HR}=2.21(95 \% \mathrm{Cl}=1.09-4.48$, $\mathrm{p}=0.03)$ in a second population-based study $(\mathrm{n}=151)$.

CHFR promoter CpG island methylation, which is associated with MSI, also occurs frequently in MSS CRCs and is a promising prognostic marker in stage II, MSS, BRAF wild-type CRCs. 
Promoter CpG island methylation and prognosis in colorectal cancer $\mid 69$

\section{Introduction}

Accurate staging of colorectal cancer (CRC) is essential for optimal disease management. Although patients with the same stage can demonstrate considerable variation in outcome, the TNM staging system remains the gold standard for predicting prognosis and guiding clinical management of $C R C^{1}$.

Adjuvant chemotherapy is recommended for all stage III CRC patients. In Europe, the majority of stage II CRC patients undergo surgery alone, despite the recognition that a subgroup with a poor prognosis would probably benefit from adjuvant chemotherapy. Molecular classification of CRC might aid in selecting CRC patients who could benefit from adjuvant therapy.

CRC is characterized by (epi)genetic alterations of genes controlling the hallmarks of cancer ${ }^{2,3-5}$. Frequently observed alterations affecting these pathways include chromosomal instability $(\mathrm{CIN})$, microsatellite instability (MSI), coding sequence mutations in APC, TP53, KRAS and $P I 3 K C A^{3-5}$ and promoter $\mathrm{CpG}$ island hypermethylation. A distinct subset of CRCs, characterized a greater degree of promoter $\mathrm{CpG}$ island methylation, is associated with proximal location, poor differentiation, $\mathrm{MSI}$ and $B R A F$ mutations ${ }^{6-11}$ and is referred to as the $\mathrm{CpG}$ island methylator phenotype $(\mathrm{CIMP})^{10,12}$.

CIMP has previously been associated with CRC prognosis. However, published reports are inconsistent, perhaps due to confounding factors such as $\mathrm{MSI}, B R A F$ and KRAS mutations, variations in use of adjuvant chemotherapy ${ }^{13-16}$, and methodological differences, such as different CIMP definitions.

The aim of this study was to evaluate the role of CIMP and frequently methylated promoter $\mathrm{CpG}$ islands on prognosis of CRC, eliminating the influence of genetic alterations and adjuvant chemotherapy.

\section{Materials and methods}

\section{Study population}

CRC patients were entered in two multi-center prospective clinical trials between 1979 and 1981 in the Netherlands. One trial was designed to compare patient survival after treatment of colon cancer by conventional surgery or the no-touch isolation technique ${ }^{17}$. The second trial was conducted to compare survival in rectal cancer patients with or without preoperative radiotherapy, of which we included only the patients in the no preoperative radiotherapy arm. At the time the trial was conducted, adjuvant chemotherapy was not yet standard practice. Tumor stage was defined according to the UICC-TNM staging system and American Joint Committee on Cancer classifications (AJCC), Cancer Staging Sixth Edition ${ }^{18,19}$. For both trials, follow up took place every 3 months 
$70 \mid$ Chapter 4

during the first three years and every 6 months between three and five years after initial diagnosis and surgery. Standard protocols were followed, with routine blood counts and chemistry studies (including CEA levels) at each visit and liver ultrasound, chest $\mathrm{x}$-ray and colonoscopy annually, to evaluate recurrence of disease and disease-related death. After five years of follow up, only time and cause of death were registered. Follow-up was complete for all patients. Failure was defined as death due to recurrent disease, excluding postoperative mortality within 30 days and non-disease related death. For molecular analyses, tumor tissues from 173 patients with primary CRC were available.

\section{Independent, validation population of CRCs}

A second, independent population of 734 CRC cases, derived from the prospective Netherlands Cohort Study on diet and cancer which started in 1986 with the enrolment of 120,852 healthy individuals between 55 and 69 years old from 204 municipalities throughout the Netherlands, was used to validate survival data. From 1989 until 1994, 925 incident CRC cases (ICD-O:153.0154.1) were identified by computerized linkage with the Netherlands Cancer Registry and PALGA, a nationwide network and registry of histopathology and cytopathology ${ }^{20}$. Information on tumor localization, tumor staging, differentiation grade and incidence date was available through the Netherlands Cancer Registry. Vital status until May 2005 was retrieved from the Central Bureau of Genealogy and the municipal population registries and could be obtained for all cases. Causes of death were retrieved through linkage with Statistics Netherlands. Paraffin-embedded tumor tissue was collected from 54 pathology registries; tissue blocks for $734(90 \%)$ of the CRC cases contained sufficient DNA for analyses. Details of this cohort have been described previously ${ }^{21}$.

Clinical pathological characteristics are provided for both populations (Table $4.1)$. In the validation study more left-sided tumors $(43 \%)(p<0.01)$ and stage I tumors $(27 \%) \quad(p<0.01)$ were diagnosed and the median follow up time was longer in the validation population: 7.6 years compared to 4.8 years. $p<0.01$ ). However, event frequencies were comparable between both studies, making the validation population suitable for validation of prognostic markers. 
Promoter CpG island methylation and prognosis in colorectal cancer $\mid 71$

Table 4.1 Clinicopathological characteristics of the CRC populations

\begin{tabular}{lccc}
\hline & $\begin{array}{c}\text { Study population } \\
(\mathrm{n}=173)\end{array}$ & $\begin{array}{c}\text { Validation population } \\
(\mathrm{n}=734)\end{array}$ & $\mathrm{p}$-value \\
\hline $\begin{array}{l}\text { Age } \\
\text { Mean age (SD) }\end{array}$ & $67.80(11.8)$ & $62.90(4.1)$ & $\mathrm{NS}$ \\
$\begin{array}{l}\text { Gender } \\
\quad \text { Male }\end{array}$ & $82(47 \%)$ & $408(56 \%)$ & $\mathrm{NS}$ \\
$\quad$ Female & $91(53 \%)$ & $326(44 \%)$ & \\
Tumor location & & & \\
$\quad$ Right-sided colon & $62(36 \%)$ & $239(33 \%)$ & $<0.01$ \\
Left-sided colon & $52(30 \%)$ & $310(43 \%)$ & \\
Rectum & $59(34 \%)$ & $176(24 \%)$ & \\
CRC Stage & $4(2 \%)$ & $181(27 \%)$ & $<0.01$ \\
I & $100(58 \%)$ & $236(35 \%)$ & \\
II & $50(29 \%)$ & $185(28 \%)$ & \\
III & $19(11 \%)$ & $69(10 \%)$ & \\
IV & $64(38 \%)$ & $302(41 \%)$ & $\mathrm{NS}$ \\
Event frequency & & & \\
Median follow up time & 4.80 years & 7.60 years & $<0.01$ \\
\hline
\end{tabular}

SD: Standard Deviation, ${ }^{* *}$ colorectal cancer specific death

\section{Promoter CpG island methylation-, MSI- and BRAF and KRAS analysis}

Genomic DNA was extracted from CRC tissues using PureGene ${ }^{\mathrm{TM}}$ Genomic DNA Isolation Kit (Gentra Systems) according to the manufacturer's protocol.

Promoter $\mathrm{CpG}$ island methylation of genes reported to be methylated in colorectal cancer: mutL homolog1, colon cancer, nonpolyposis type 2 (E.Coli) (MLH1), cyclin dependent kinase inhibitor 2A (p16INK4 and p14ARF ), O-6methylguanine-DNA methyltransferase (MGMT), Ras association (RalGDS/AF6) domain family member 1 (RASSF1A), adenomatous polyposis coli (APC), helicase-like transcription factor (HLTF), GATA binding protein 4 (GATA4), GATA binding protein 5 (GATA5), checkpoint with forkhead and ring finger domains (CHFR), ADAM metallopeptidase domain 23 (ADAM23), Rab32, member RAS oncogene family (RAB32), junctophilin (3JPH3), forkhead box L2 (FOXL2), BCL2-adenovirus E1B 19kDA interacting protein 3 (BNIP3) , neuralized homolog (Drosophila) (NEURL), calcium channel, voltage dependent, alpha2-delta subunit 1 (CACNA2), thrombospondin 1 (THBS1), tissue factor pathway inhibitor 2 (TFPI2) and the CIMP genes calcium channel, voltage-dependent, $T$ type, alpha-1G subunit (CACNA1G), insulin-like growth factor 2 (somatomedin A) (IGF2), neurogenin 1 (NEUROG1), runt-related transcription factor 3 (RUNX3) and suppressor of cytokine signaling 1 (SOCS1) was determined using sodium bisulfite modification of genomic DNA (EZ DNA methylation kit, ZYMO research Co., Orange, CA). To facilitate MSP analysis on DNA retrieved from formalin-fixed, paraffin-embedded tissue, nested 
Methylation Specific PCR (MSP) was performed as described elsewhere ${ }^{22,23}$. Primers and PCR conditions are provided in Supplementary Table 4.S3.

Microsatellite instability (MSI) was determined by a pentaplex PCR, using the mononucleotide MSI markers BAT-26, BAT-25, NR-21, NR-22 and NR-24, as previously described ${ }^{24}$. MSI was defined positive when three or more of five markers (BAT-26, BAT-25, NR-21, NR-22 and NR-24) showed allelic size variants.

The common V600E BRAF mutation in exon 15 was analyzed by semi-nested PCR and subsequent RFLP analysis, KRAS mutations were analyzed as described previously ${ }^{25,26}$.

\section{Data analysis}

CIMP was analysed using the marker panel proposed by Weisenberger et al. ${ }^{11}$. CRCs were defined as CIMP when $>3 / 5$ analyzed markers (CACNA1G, IGF2, NEUROG1, RUNX3, SOCS1) were methylated. Unsupervised clustering

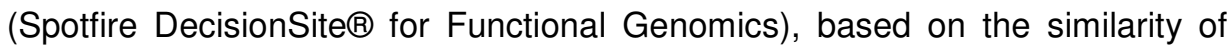
methylation of $19 \mathrm{CpG}$ islands that have been reported to be methylated in colorectal cancer (CIMP genes excluded), was performed by using half square euclidian distance (Wards method linkage rule) ${ }^{27,28}$. Methylation Index ( $\mathrm{Ml}=$ number of methylated promoter $\mathrm{CpG}$ islands divided by number of promoter $\mathrm{CpG}$ islands successfully analyzed) was calculated using the promoter CpG islands of 19 tumor suppressor- and DNA repair genes as well as the CIMP panel. Standard Error of Skewness was used as a test of symmetry for the distribution of CRC cases according to the number of methylated CIMP genes and number of methylated cluster genes, symmetry was rejected if the ratio was less than -2 or greater than +2 . Differences between methylation-, clinicopathological- and molecular characteristics were determined by the Pearson Chi-Square and Fisher's exact test where appropriate. Kaplan-Meier curves were used to evaluate the relationship between promoter $\mathrm{CpG}$ island methylation and patient survival in the overall population and stratified for tumor stage, MSI and BRAF mutation status. Statistical differences between groups were assessed by use of the log-rank test. The endpoint for analyses was overall survival starting from the day of surgery until the time of death due to $\mathrm{CRC}$. Independent variables predicting survival were evaluated in a multivariate model using Cox Regression analyses. The Cox-regression model including CIMP, CL1, CL2, CL3, CHFR promoter $\mathrm{CpG}$ island methylation, age, gender, tumor location, differentiation grade and TNM Stage was used to assess the prognostic influence of these variables. All $\mathrm{p}$ values (two sided) $<0.05$ were considered statistically significant. SPSS 15.0 and Stata 10.0 were used for data analyses. 


\section{Results}

\section{(Epi)genetic characterization of CRCs}

MSI, BRAF and KRAS mutations were detected in 11\% (19/169), 9\% (14/161) and $26 \%(41 / 157)$ of CRCs of the study population respectively (Table 4.S1), which is in accordance with previously reported frequencies ${ }^{4,5,29}$.

The methylation frequency for CIMP genes ranged from $13 \%$ to $61 \%$ : IGF2 (13\% (20/154)), CACNA1G 18\% (30/170)), RUNX3 (32\% (52/163)), NEUROG1 $42 \%(70 / 165)$ and SOCS1 (61\% (103/170)) (Figure 4.1A). 23\% (39/168) of CRCs were classified as CIMP (Table 4.S2). This is consistent with previous publications with reported frequencies of CIMP between $18-25 \%^{11,30,31}$. The distribution of the number of CIMP markers methylated for each tumor resembles a normal distribution (Figure $4.1 \mathrm{C}$, standard error of skewness $0.187)$.
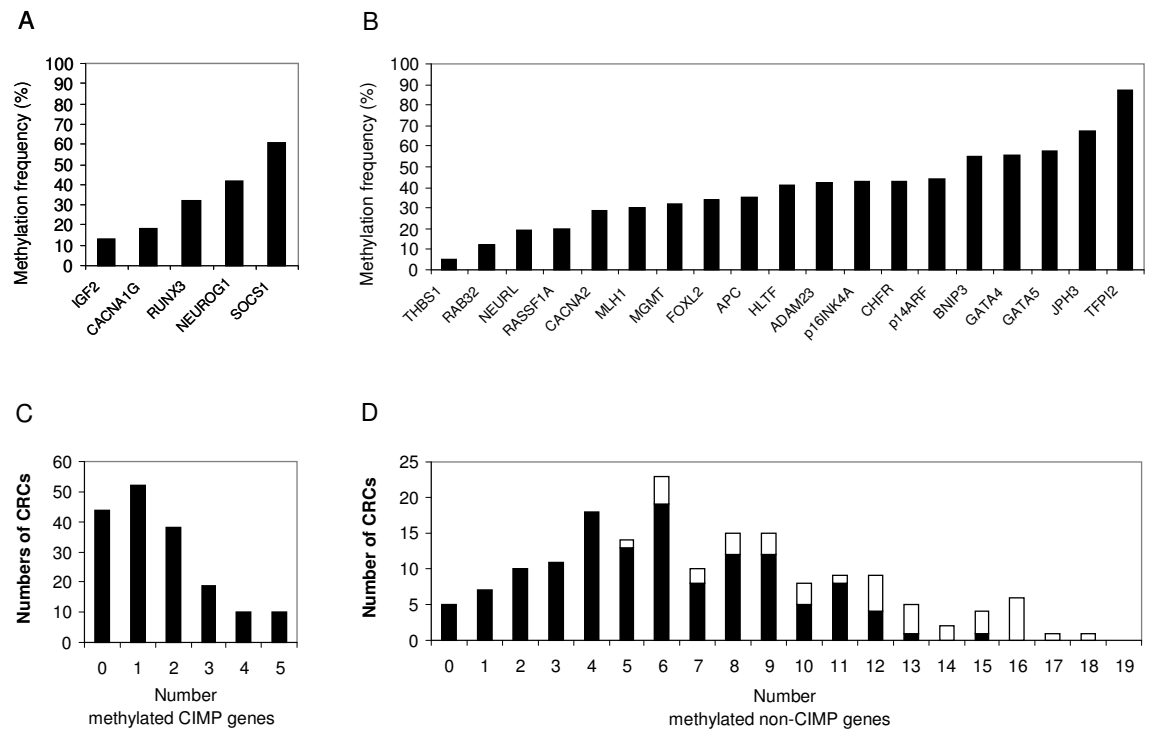

Figure 4.1 (A) Methylation frequencies of the five CIMP genes among the study-population (black bars indicate percentages). (B) Methylation frequencies of 19 cluster genes among the study-population (black bars indicate percentages). (C) Distribution of colon cancer cases according to the number of methylated CIMP genes (black bars indicate number of cases). (D) Distribution of colon cancer cases according to the number of methylated cluster genes (black bars indicate number of cases CIMP-, white bars indicate number of cases $\mathrm{CIMP}+$ ).

We sought to more broadly examine the methylation of $\mathrm{CpG}$ islands in CRC, to determine whether the 5 genes used to define CIMP optimally separated phenotypic differences in colon tumors. The frequency of promoter $\mathrm{CpG}$ island 
methylation in 19 additional tumor suppressor genes ranged from 5\% (THBS1) to $87 \%$ (TFPI2) (Figure 4.1B and Table 4.S1). We compared the distribution of tumors according to the methylation status of the 19 additional genes (Figure 4.1D). This resembles a normal distribution with a standard error of skewness 0.185 , and reveals that CRCs with a high number of methylated genes are predominantly characterized by CIMP (white bars). This suggests that the Weisenberger CIMP markers are sensitive but not completely specific in identifying $\mathrm{CRCs}$ with a high frequency of promoter $\mathrm{CpG}$ island methylation.

We assessed whether subgroups of CRCs could be identified using methylation patterns of the 19 non-CIMP genes. Unsupervised hierarchical cluster analysis identified three clusters of CRCs, CL1 (57\% (98/173)), CL2 (25\% (43/173)) and CL3 (18\% (32/173)) (Figure 4.2, Table 4.S2). CL3 showed the highest number of methylated genes (MI) (mean MI, CL1 $=0.25, C L 2=0.49$, $\mathrm{CL} 3=0.69, \mathrm{p}=<0.001)$.
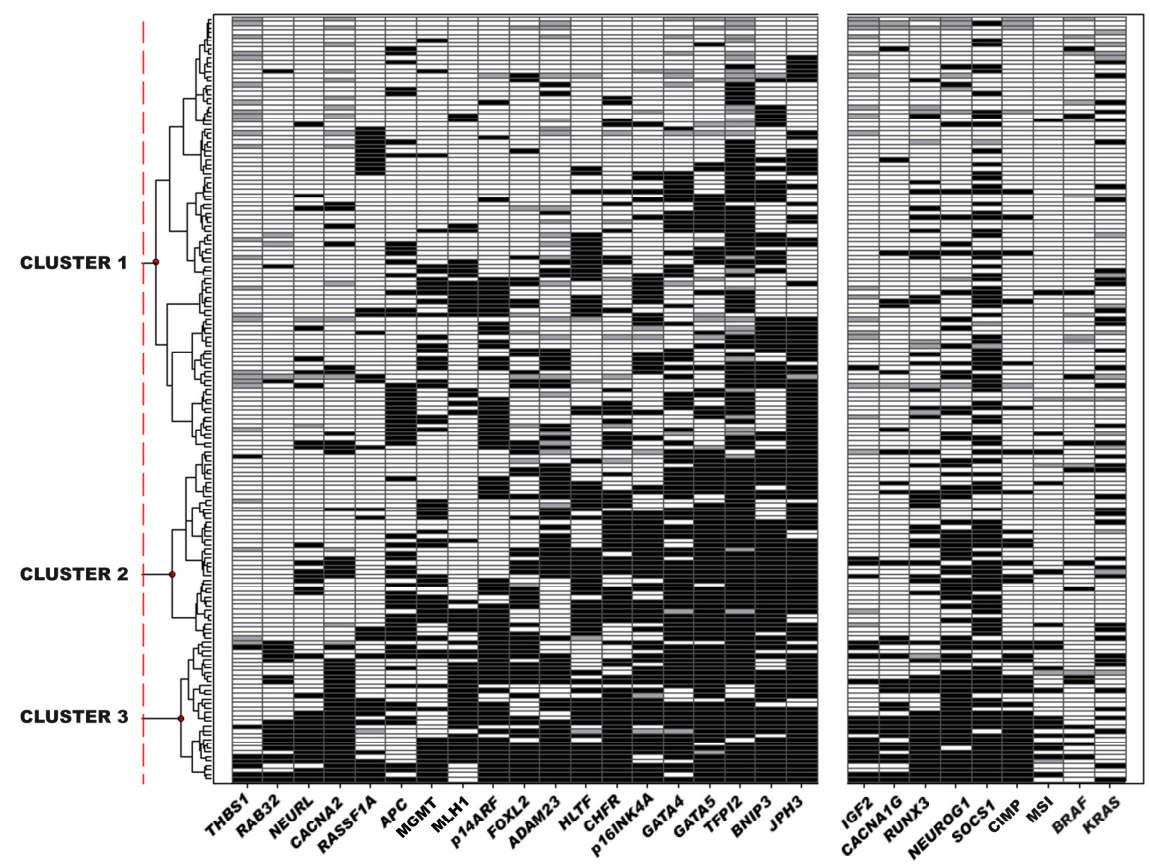

Figure 4.2 CRCs clusters CL1 (57\%), CL2 (25\%), CL3 (18\%) obtained by unsupervised hierarchical clustering of promoter $\mathrm{CpG}$ island methylation of $M L H 1, p 16 I N K 4 A$, p14ARF, MGMT, RASSF1A, APC, HLTF, GATA4, GATA5, CHFR, ADAM23, RAB32, JPH3, FOXL2, BNIP3, NEURL, CACNA2, THBS1, TFPI2.

Black box indicates methylated gene, white box indicates unmethylated gene, grey box indicates failed PCR. After clustering, identification of patients was done for individual CIMP markers (CACNA1G, IGF2, NEUROG1, RUNX3 and SOCS1), CIMP, MSI, BRAF- and KRAS mutations as visualized. Black box indicates positive, white box indicates negative, grey box indicates missing value. 
Promoter CpG island methylation and prognosis in colorectal cancer $\mid 75$

\section{Associations between genetic, epigenetic and clinicopathological characteristics}

CIMP was highly associated with promoter CpG island methylation of $16 / 19$ additional genes. Furthermore, CRCs grouped in CL3 were most often classified as CIMP $(p<0.01)$ (Figure 4.2, Table 4.2). CIMP was associated with MSI, BRAF mutations and CL3 ( $\mathrm{p}<0.01, \mathrm{p}=0.05$ and $\mathrm{p}<0.01$ respectively) (Table 4.2). 69\% (26/38) of CIMP CRCs and 60\% (18/30) of CL3 CRCs are MSS (Table 4.2). Mutations in KRAS were neither associated with CIMP nor with the identified clusters (data not shown).

Table 4.2 Correlations between CIMP, CL1, CL2 and CL3 and MSI and BRAF mutation in the study population.

\begin{tabular}{lccccc}
\hline & MSI & MSS & BRAF $\boldsymbol{B}$ & BRAF WT & CIMP \\
\hline CIMP & $12 / 19$ & $26 / 145$ & $7 / 14$ & $32 / 143$ & \\
& $(63 \%)$ & $(18 \%)$ & $(50 \%)$ & $(22 \%)$ & \\
& & $<0.01$ & & $<0.05$ & \\
CL1 & $3 / 19$ & $94 / 150$ & $5 / 14$ & $83 / 147$ & $6 / 39$ \\
& $(16 \%)$ & $(63 \%)$ & $(36 \%)$ & $(57 \%)$ & $(15 \%)$ \\
CL2 & $4 / 19$ & $38 / 150$ & $3 / 14$ & $39 / 147$ & $8 / 39$ \\
& $(21 \%)$ & $(25 \%)$ & $(21 \%)$ & $(27 \%)$ & $(21 \%)$ \\
& $12 / 19$ & $18 / 150$ & $6 / 14$ & $25 / 147$ & $25 / 39$ \\
& $(63 \%)$ & $(12 \%)$ & $(43 \%)$ & $(17 \%)$ & $(64 \%)$ \\
& & $<0.01$ & & 0.06 & $<0.01$ \\
\hline
\end{tabular}

CIMP: CpG island methylation phenotype using the markers of Weisenberger et al. ${ }^{1}, \mathrm{CL} 1, \mathrm{CL} 2$ and CL3: groups of CRCs identified by unsupervised hierarchical clustering based on methylation patterns of 19 tumor suppressor and DNA repair genes, MSI: microsatellite instability, MSS: microsatellite stability, M: Mutant, WT: Wild-type.

As expected, CIMP and MSI were associated with right-sided tumor location, as were CL3 CRCs $(p=<0.01, p=<0.01$ and $p=<0.01$ respectively, Tables 4.S1 and S2). We did not observe previously reported association between sex, age and CIMP or with our newly defined CL3 tumors.

The primary aim of this study was to evaluate the prognostic influence of promoter $\mathrm{CpG}$ island methylation using CIMP- and to extend this analysis to include 19 additional tumor suppressor- and DNA repair genes. As tumor stage, MSI, BRAF- and KRAS mutations have in some reports influenced survival, we analyzed whether these alterations were of prognostic significance in the study population. As expected, disease outcome was significantly influenced by tumor stage, with stage I having an improved overall survival $(p<0.01)$ compared to other tumor stages. We also observed that wild-type $B R A F$ tumors showed an improved overall survival compared to $B R A F$ mutated tumors $(\mathrm{p}=0.04)$. MSI seems associated with improved overall survival as compared to MSS within CRC Stage II, although this association is not 
$76 \mid$ Chapter 4

statistically significant $(\mathrm{p}=0.07)$. KRAS mutations were of no prognostic value, neither in the overall population, nor in the separate stages (data not shown). Therefore, survival analyses were also stratified for stage, MSI status and $B R A F$ mutation status.

No statistically significant survival differences between CIMP and non-CIMP CRCs were observed in the overall study population (Figure 4.3A) or when specifically examining Stage II (Figure 4.3C,) or Stage III (data not shown) MSS, BRAF wild type CRCs. The same was observed for CL1, CL2 and CL3 CRCs (data not shown). Examining the validation population showed that overall CIMP was borderline statistically significantly associated with prognosis (Figure 4.3B, $p=0.02$ ). This finding was lost within Stage II (Figure 4.3D) and Stage III (data not shown) MSS, BRAF wild type CRCs. We next sought to determine whether one possible explanation for additional variation in previous studies was that specific CpG island methylation, underlying any prognostic importance, was imperfectly associated with CIMP. When analyzing all 19 (or 24 if including CIMP) genes, only $A P C$ and CHFR methylation, although the latter was positively associated with MSI $(p=0.02)$ and CIMP $(p<0.01$, Table 4.S4), were associated with a worse prognosis in stage II MSS, BRAF wild-type CRCs $(\mathrm{HR}=2.63(95 \% \mathrm{Cl}=1.21-5.68, \mathrm{p}=0.01)$ and $\mathrm{HR}=2.59(95 \% \mathrm{Cl}=1.16-$ $5.76, p=0.02$ ) respectively) (Table $4 . S 5$ and Figure $4.3 \mathrm{G}$ ). For stage III MSS $B R A F$ wildtype CRCs, associations with worse prognosis were found for RASSF1A, THBS1 and CACNA1G (HR=3.89 (95\% Cl 1.23-12.3, $\mathrm{p}=0.02$; $\mathrm{HR}=26.5(95 \% \mathrm{Cl}=1.66-423, \mathrm{p}=0.02)$ and $\mathrm{HR}=5.62 \quad(95 \% \mathrm{Cl}=1.49-21.3$, $\mathrm{p}=0.01)$ ) (Table 4.S5). These effects were not observed in the overall study population (Table 4.S5). Since the small numbers of cases and the number of comparisons examined to find significance raises concerns, we validated these results in an independent validation population. This only confirmed a statistically significant association with prognosis for $C H F R$ methylation in stage II, MSS, BRAF wild-type CRCs (Figure 4.3F-3H). The Cox-regression multivariate model within the study population of MSS, BRAF wild-type, stage II CRCs shows that CHFR methylation was associated with a poor prognosis ( $\mathrm{HR}=3.89(95 \% \mathrm{Cl}=1.58-9.60), \mathrm{p}=<0.01$ and was, in this study, a better predictor of survival than differentiation grade (HR $1.52(95 \% \mathrm{Cl}=0.69-3.36)$, $\mathrm{p}=0.30$ ) (Table 4.3). Multivariate analysis in the independent, population-based validation cohort of 151 stage II, MSS, BRAF wildtype CRCs confirmed that CHFR methylation is associated with a worse prognosis in this specific subgroup of CRCs $(n=151, \mathrm{HR}=2.21(95 \% \mathrm{Cl}=1.09-4.48, \mathrm{p}=0.03)$ (Table 4.3). 
A
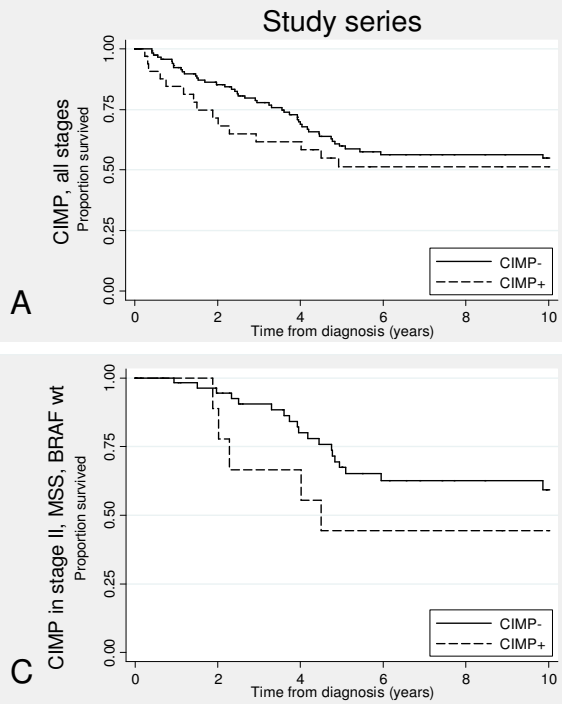

$E$
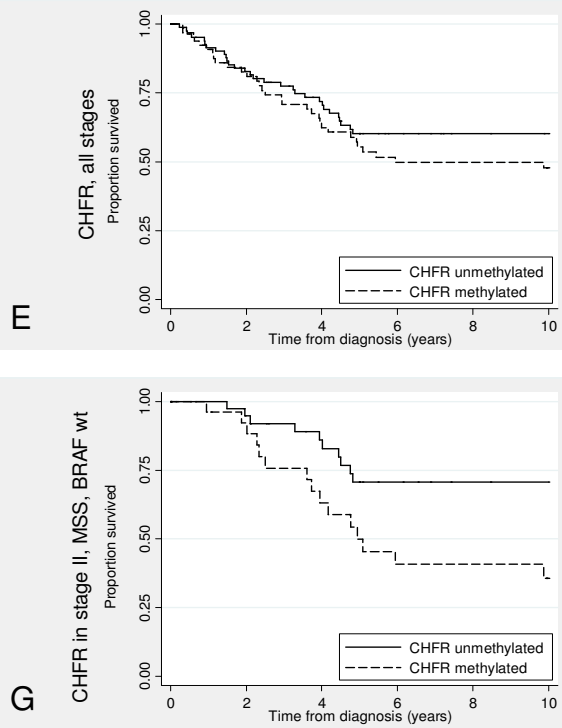
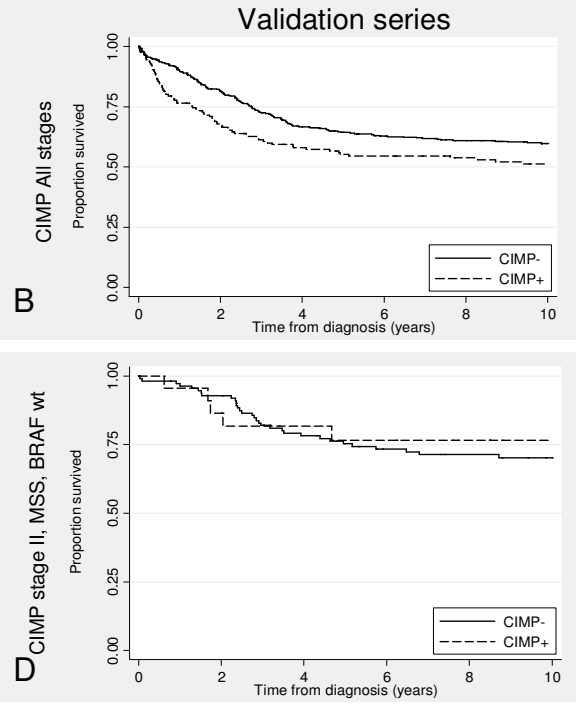

$\mathrm{F}$
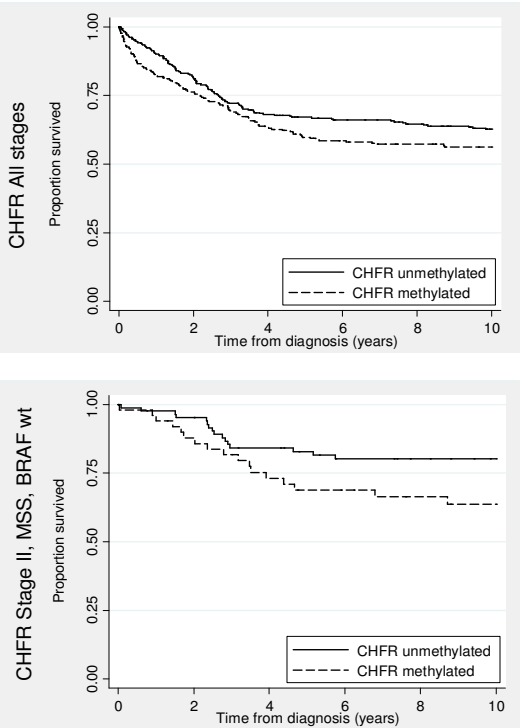

Figure 4.3 Survival curves overall population of (A) CIMP- $(n=124)$ versus CIMP+ $(n=38)$, $p=0.381$; of validation series (B) CIMP- $(n=436)$ versus CIMP+ $(n=167), p=0.016$. Survival curves in stage II, MSS and BRAF wild-type tumors of $(C) C I M P+(n=12)$ and CIMP- $(n=61), p=0.158$; of validation series (D) CIMP+ $(n=23)$ and CIMP- $(n=113)$, $\mathrm{p}=0.701$. Survival curves overall population of $(E) C H F R$ unmethylated $(n=89)$ versus CHFR methylated $(\mathrm{n}=71), \mathrm{p}=0.259$; of validation series $(\mathrm{F})$ CHFR unmethylated $(\mathrm{n}=319)$ versus $C H F R$ methylated $(\mathrm{n}=280), \mathrm{p}=0.078$. Survival curves in stage II, MSS and $B R A F$ wild-type tumors of $(G) C H F R$ unmethylated $(n=43)$ and $C H F R$ methylated $(\mathrm{n}=30)$ CRCs, $\mathrm{p}=0.016$; of validation series $(\mathrm{H})$ CHFR unmethylated $(\mathrm{n}=85)$ and CHFR methylated $(n=50)$ CRCs, $p=0.049$. 
$78 \mid$ Chapter 4

Table 4.3 Cox proportional hazard models for MSS, BRAF wild-type, stage II CRCs

\begin{tabular}{|c|c|c|c|c|}
\hline & \multicolumn{2}{|c|}{ Study population $(n=66)$} & \multicolumn{2}{|c|}{ Validation population $(n=151)$} \\
\hline & $\begin{array}{c}\text { CRC mortality } \\
\text { HR }(95 \% \mathrm{Cl})\end{array}$ & $p$ value & $\begin{array}{l}\text { CRC mortality } \\
\mathrm{HR}(95 \% \mathrm{Cl})\end{array}$ & $p$-value \\
\hline CHFR $\mathrm{M}^{*}$ & $3.89(1.58-9.60)$ & $<0.01$ & $2.21(1.09-4.48)$ & 0.03 \\
\hline Age (years) & $1.02(0.97-1.07)$ & 0.41 & $1.02(0.94-1.11)$ & 0.43 \\
\hline Gender ( female ${ }^{* *}$ ) & $0.53(0.23-1.27)$ & 0.16 & $1.32(0.66-2.63)$ & 0.62 \\
\hline Tumor location & & & & \\
\hline Left-sided Colon ${ }^{+}$ & $0.52(0.20-1.38)$ & 0.19 & $1.19(0.51-2.78)$ & 0.69 \\
\hline Rectum $^{+}$ & $0.29(0.09-0.93)$ & 0.04 & $1.89(0.64-5.52)$ & 0.25 \\
\hline Differentiation grade (poor $\left.{ }^{\#}\right)$ & $1.52(0.69-3.36)$ & 0.30 & $0.70(0.21-2.36)$ & 0.56 \\
\hline
\end{tabular}

${ }^{*}$ Reference group $=$ CHFR unmethylated cases; ${ }^{*}$ Reference group $=$ male, ${ }^{+}$Reference group = Right-sided colon, "Reference group $=$ Well $/$ moderate. HR: Hazard Ratio=Relative Risk, $M$ : Methylated.

\section{Discussion}

Data on the prognostic significance of promoter $\mathrm{CpG}$ island methylation, and CIMP in particular, are conflicting in $\mathrm{CRC}^{13-16}$. These inconsistencies might be caused by factors affecting the course of the disease, such as genetic alterations, adjuvant therapy and differences in methodological study approaches. Here we analysed the prognostic value of promoter $\mathrm{CpG}$ island methylation in CRCs from patients not treated with (neo)adjuvant therapy taking into account the confounding role of clinicopathological and genetic (MSI and BRAF) characteristics. Previously reported associations between CIMP and proximal tumor location, MSI and BRAF mutations ${ }^{10,11,16}$ as well as the identification of 3 subgroups of $\mathrm{CRCs}^{9,32}$ based on promoter methylation profiles could be confirmed. In addition, the prognostic role of tumor stage, MSI and $B R A F^{33,34}$ could be confirmed in the study and the validation series.

In the study series, neither CIMP nor CL3 classification were statistically significantly associated with prognosis, neither overall nor when stratified for stage or microsatellite status. These conclusions are valid for the subgroups of CRC defined by analyzing CIMP with the markers proposed by Weisenberger et al. ${ }^{11}$ and also for unsupervised clustering of the methylation data for the 19 CpG islands that we added to the analysis. Since also other CIMP definitions have been used, caution with generalizing these conclusions is warranted. However, these data confirm the concept that based on $\mathrm{CpG}$ island methylation, 3 subgroups of CRC can be identified, independently of the specific markers used. The subgroup characterized by extensive promoter $\mathrm{CpG}$ island methylation is strongly, although not perfectly, associated with CIMP and its reported clinicopathological characteristics. The borderline overall statistically significant prognostic effect of CIMP in the validation series indicates that the prognostic role of CIMP in CRC is still unclear and that large, 
independent studies are needed to answer this question. In addition, until the underlying biological cause for CIMP is being identified, an accurate definition remains hard to establish. Our evidence for imperfect correlation of highly methylated tumors using the independent 19 genes with CIMP illustrates this.

After stratification for established confounders including age, gender, tumor location, differentiation grade, TNM Stage, MSI and BRAF mutation status, promoter methylation of only 1 of 24 genes, namely CHFR, was statistically significantly associated with a poor prognosis in stage II MSS CRCs in the study population $(\mathrm{HR}=3.89(95 \% \mathrm{Cl}=1.58-9.602), \mathrm{p}=0.003)$ and the validation series $(\mathrm{HR}=2.21(95 \% \mathrm{Cl}=1.09-4.48, \mathrm{p}=0.03)$. Because of the assumption that many biomarker data are false ${ }^{35}$ and the fact that we studied many variables, our validation of the effect of CHFR promoter $\mathrm{CpG}$ island methylation in an independent prospective cohort study as well as pilot data recently published by an independent team pointing to the same conclusion ${ }^{36}$, underscores the potential of $C H F R$ promoter $\mathrm{CpG}$ island methylation as a prognostic marker in colorectal cancer.

A dominant effect of MSI over DNA methylation regarding prognosis has been shown previously in CRC by Ward et al., for a 'CIMP'-like' phenotype ${ }^{37}$. The mechanisms underlying this paradox are still not clear. An explanation could be the increased lymphocytic infiltrate that is observed in MSI CRCs ${ }^{38}$ or the extend of instability making MSI tumor cells less fit to metastasize ${ }^{39,40}$. The prognostic effect of CHFR in MSS stage II CRCs is surprising, as we previously reported a strong association between $C H F R$ promoter $\mathrm{CpG}$ island methylation, MSI and MLH1 promoter methylation in $\mathrm{CRC}^{41}$. However, since a significant subset of MSS CRCs also have CHFR promoter methylation, this could be of clinical relevance.

CHFR is a tumor suppressor gene which is inactivated by promoter CpG island methylation in a variety of solid tumors ${ }^{41-45}$. It encodes an ubiquitin ligase that regulates both entry into metaphase and chromosome segregation later in mitosis to maintain genomic stability ${ }^{46,47}$. CHFR inactivation has been hypothesized to be associated with chromosomal instability, although conflicting data have been reported ${ }^{48-50}$. Although both $C H F R$ and $M L H 1$ contribute to genomic integrity, they function through different mechanisms. CHFR deficiency triggers mild CIN and $h M L H 1$ deficiency leading to $\mathrm{MSI}^{51}$. Recent data from $\mathrm{Oh}$ et al $2009^{52}$ indicate that, in vitro, CHFR binds and downregulates HDAC1 thereby downregulating the Cdk inhibitor p21 ${ }^{\text {CIP/WAF1 }}$ and the metastasis suppressors KAI1 and E-cadherin. This eventually results in cell cycle arrest and a less invasive phenotype. In addition to a potential role for CHFR as prognostic biomarker, CHFR promoter methylation has been proposed as biomarker for response to microtubule inhibitor taxanes in endometrial- ${ }^{53}$ cervical $^{53}$, oral ${ }^{54}$ and gastric cancer ${ }^{55}$. Although taxanes are not implemented in CRC treatment because they failed to demonstrate a significant 
clinical benefit in Phase II trials ${ }^{56,57}$, CRCs with CHFR promoter methylation might benefit from taxanes.

In summary, although our study failed to demonstrate a consistent prognostic effect of CIMP, we identified promoter $\mathrm{CpG}$ island methylation of $\mathrm{CHFR}$ as a prognostic biomarker in stage II, MSS, BRAF wild-type CRCs in two independent populations.

Future large-scale validation studies and randomized clinical trials are needed to study the clinical value CHFR methylation in CRC. 
Promoter CpG island methylation and prognosis in colorectal cancer $\mid 81$

\section{References}

1. Wallner $M$, Herbst $A$, Behrens $A$, et al: Methylation of serum DNA is an independent prognostic marker in colorectal cancer. Clin Cancer Res 12:7347-52, 2006

2. Hanahan D, Weinberg RA: The hallmarks of cancer. Cell 100:57-70, 2000

3. Schuebel KE, Chen W, Cope L, et al: Comparing the DNA hypermethylome with gene mutations in human colorectal cancer. PLoS Genet 3:1709-23, 2007

4. Sjoblom T, Jones S, Wood LD, et al: The consensus coding sequences of human breast and colorectal cancers. Science 314:268-74, 2006

5. Wood LD, Parsons DW, Jones S, et al: The genomic landscapes of human breast and colorectal cancers. Science 318:1108-13, 2007

6. Ogino S, Cantor M, Kawasaki T, et al: $\mathrm{CpG}$ island methylator phenotype (CIMP) of colorectal cancer is best characterised by quantitative DNA methylation analysis and prospective cohort studies. Gut 55:1000-6, 2006

7. Ogino S, Kawasaki T, Kirkner GJ, et al: Evaluation of markers for CpG island methylator phenotype (CIMP) in colorectal cancer by a large population-based sample. J Mol Diagn 9:305-14, 2007

8. Samowitz WS, Albertsen H, Herrick J, et al: Evaluation of a large, population-based sample supports a CpG island methylator phenotype in colon cancer. Gastroenterology 129:837-45, 2005

9. Shen L, Toyota M, Kondo $\mathrm{Y}$, et al: Integrated genetic and epigenetic analysis identifies three different subclasses of colon cancer. Proc Natl Acad Sci U S A 104:18654-9, 2007

10. Toyota M, Ahuja N, Ohe-Toyota $M$, et al: $C p G$ island methylator phenotype in colorectal cancer. Proc Natl Acad Sci U S A 96:8681-6, 1999

11. Weisenberger DJ, Siegmund KD, Campan M, et al: CpG island methylator phenotype underlies sporadic microsatellite instability and is tightly associated with BRAF mutation in colorectal cancer. Nat Genet 38:787-93, 2006

12. Hamilton SR: Targeted therapy of cancer: new roles for pathologists in colorectal cancer. Mod Pathol 21 Suppl 2:S23-30, 2008

13. Ogino S, Meyerhardt JA, Kawasaki T, et al: $\mathrm{CpG}$ island methylation, response to combination chemotherapy, and patient survival in advanced microsatellite stable colorectal carcinoma. Virchows Arch 450:529-37, 2007

14. Samowitz WS, Sweeney C, Herrick J, et al: Poor survival associated with the BRAF V600E mutation in microsatellite-stable colon cancers. Cancer Res 65:6063-9, 2005

15. Shen L, Catalano PJ, Benson AB, 3rd, et al: Association between DNA methylation and shortened survival in patients with advanced colorectal cancer treated with 5-fluorouracil based chemotherapy. Clin Cancer Res 13:6093-8, 2007

16. Ogino S, Nosho K, Kirkner GJ, et al: $\mathrm{CpG}$ island methylator phenotype, microsatellite instability, BRAF mutation and clinical outcome in colon cancer. Gut 58:90-6, 2009

17. Wiggers $\mathrm{T}$, Jeekel $\mathrm{J}$, Arends JW, et al: No-touch isolation technique in colon cancer: a controlled prospective trial. Br J Surg 75:409-15, 1988

18. AJCC: Cancer staging manual. 2002

19. Sobin L, Wittekind C: TNM classification of malignant tumours. 2002

20. Van den Brandt PA, Schouten LJ, Goldbohm RA, et al: Development of a record linkage protocol for use in the Dutch Cancer Registry for Epidemiological Research. Int J Epidemiol 19:553-8, 1990

21. van den Brandt PA, Goldbohm RA, van 't Veer $P$, et al: A large-scale prospective cohort study on diet and cancer in The Netherlands. J Clin Epidemiol 43:285-95, 1990

22. Herman JG, Graff JR, Myohanen S, et al: Methylation-specific PCR: a novel PCR assay for methylation status of CpG islands. Proc Natl Acad Sci U S A 93:9821-6, 1996

23. Derks S, Lentjes MH, Hellebrekers DM, et al: Methylation-specific PCR unraveled. Cell Oncol 26:291-9, 2004 
$82 \mid$ Chapter 4

24. Suraweera N, Duval A, Reperant M, et al: Evaluation of tumor microsatellite instability using five quasimonomorphic mononucleotide repeats and pentaplex PCR. Gastroenterology 123:1804-11, 2002

25. Luchtenborg M, Weijenberg MP, Wark PA, et al: Mutations in APC, CTNNB1 and K-ras genes and expression of hMLH1 in sporadic colorectal carcinomas from the Netherlands Cohort Study. BMC Cancer 5:160, 2005

26. Sieben NL, Roemen GM, Oosting J, et al: Clonal analysis favours a monoclonal origin for serous borderline tumours with peritoneal implants. J Pathol 210:405-11, 2006

27. Carlis J, Bruso K: How many clusters to report: A recursive heuristic. Conf Proc IEEE Eng Med Biol Soc 1:1069-72, 2010

28. Kaushal D, Naeve CW: Loading and preparing data for analysis in spotfire. Curr Protoc Bioinformatics Chapter 7:Unit 7 8, 2004

29. Worthley DL, Whitehall VL, Spring KJ, et al: Colorectal carcinogenesis: road maps to cancer. World J Gastroenterol 13:3784-91, 2007

30. Kawasaki T, Ohnishi M, Nosho K, et al: CpG island methylator phenotype-low (CIMP-low) colorectal cancer shows not only few methylated CIMP-high-specific CpG islands, but also low-level methylation at individual loci. Mod Pathol, 2008

31. Lee S, Cho NY, Choi M, et al: Clinicopathological features of CpG island methylator phenotype-positive colorectal cancer and its adverse prognosis in relation to KRAS/BRAF mutation. Pathol Int 58:104-13, 2008

32. Derks S, Postma C, Carvalho B, et al: Integrated analysis of chromosomal, microsatellite and epigenetic instability in colorectal cancer identifies specific associations between promoter methylation of pivotal tumour suppressor and DNA repair genes and specific chromosomal alterations. Carcinogenesis 29:434-9, 2008

33. Popat S, Hubner R, Houlston RS: Systematic review of microsatellite instability and colorectal cancer prognosis. J Clin Oncol 23:609-18, 2005

34. Zlobec I, Kovac M, Erzberger P, et al: Combined analysis of specific KRAS mutation, BRAF and microsatellite instability identifies prognostic subgroups of sporadic and hereditary colorectal cancer. Int J Cancer 127:2569-75, 2010

35. Loannidis J: Why most published research findings are false. PLoS Med 2, 2005

36. Tanaka M, Chang P, Li Y, et al: Association of CHFR Promoter Methylation with Disease Recurrence in Locally Advanced Colon Cancer. Clin Cancer Res 17:4531-40, 2011

37. Ward RL, Cheong K, Ku SL, et al: Adverse prognostic effect of methylation in colorectal cancer is reversed by microsatellite instability. J Clin Oncol 21:3729-36, 2003

38. Quinn E, Hawkins N, Yip YL, et al: CD103+ intraepithelial lymphocytes--a unique population in microsatellite unstable sporadic colorectal cancer. Eur J Cancer 39:469-75, 2003

39. Lamlum $\mathrm{H}$, llyas $\mathrm{M}$, Rowan $\mathrm{A}$, et al: The type of somatic mutation at APC in familial adenomatous polyposis is determined by the site of the germline mutation: a new facet to Knudson's 'two-hit' hypothesis. Nat Med 5:1071-5, 1999

40. Tomlinson I, Bodmer W: Selection, the mutation rate and cancer: ensuring that the tail does not wag the dog. Nat Med 5:11-2, 1999

41. Brandes JC, van Engeland M, Wouters KA, et al: CHFR promoter hypermethylation in colon cancer correlates with the microsatellite instability phenotype. Carcinogenesis 26:1152-6, 2005

42. Mizuno $\mathrm{K}$, Osada $\mathrm{H}$, Konishi $\mathrm{H}$, et al: Aberrant hypermethylation of the CHFR prophase checkpoint gene in human lung cancers. Oncogene 21:2328-33, 2002

43. Shibata $\mathrm{Y}$, Haruki N, Kuwabara $\mathrm{Y}$, et al: Chfr expression is downregulated by CpG island hypermethylation in esophageal cancer. Carcinogenesis 23:1695-9, 2002

44. Corn PG, Summers MK, Fogt F, et al: Frequent hypermethylation of the 5' CpG island of the mitotic stress checkpoint gene $\mathrm{Chfr}$ in colorectal and non-small cell lung cancer. Carcinogenesis 24:47-51, 2003

45. Satoh A, Toyota $M$, Itoh $F$, et al: Epigenetic inactivation of CHFR and sensitivity to microtubule inhibitors in gastric cancer. Cancer Res 63:8606-13, 2003

46. Kang $\mathrm{D}$, Chen J, Wong $\mathrm{J}$, et al: The checkpoint protein Chfr is a ligase that ubiquitinates Plk1 and inhibits Cdc2 at the G2 to M transition. J Cell Biol 156:249-59, 2002 
47. Scolnick DM, Halazonetis TD: Chfr defines a mitotic stress checkpoint that delays entry into metaphase. Nature 406:430-5, 2000

48. Maddika S, Sy SM, Chen J: Functional Interaction between Chfr and Kif22 Controls Genomic Stability. J Biol Chem 284:12998-3003, 2009

49. Privette LM, Petty EM: CHFR: A Novel Mitotic Checkpoint Protein and Regulator of Tumorigenesis. Transl Oncol 1:57-64, 2008

50. Bertholon J, Wang Q, Falette N, et al: Chfr inactivation is not associated to chromosomal instability in colon cancers. Oncogene 22:8956-60, 2003

51. Fu Z, Regan K, Zhang L, et al: Deficiencies in Chfr and Mlh1 synergistically enhance tumor susceptibility in mice. J Clin Invest 119:2714-24, 2009

52. Oh YM, Kwon YE, Kim JM, et al: Chfr is linked to tumour metastasis through the downregulation of HDAC1. Nat Cell Biol 11:295-302, 2009

53. Banno K, Yanokura M, Kawaguchi M, et al: Epigenetic inactivation of the CHFR gene in cervical cancer contributes to sensitivity to taxanes. Int J Oncol 31:713-20, 2007

54. Ogi K, Toyota M, Mita H, et al: Small interfering RNA-induced CHFR silencing sensitizes oral squamous cell cancer cells to microtubule inhibitors. Cancer Biol Ther 4:773-80, 2005

55. Koga $Y$, Kitajima $Y$, Miyoshi A: The significance of aberrant CHFR methylation for clinical response to microtubule inhibitors in gastric cancer. J Gastroenterol 41:133-9, 2006

56. Swanton C, Tomlinson I, Downward J: Chromosomal instability, colorectal cancer and taxane resistance. Cell Cycle 5:818-23, 2006

57. Smits K, Cleven AH, Weijenberg MP, et al: Pharmacoepigenomics in colorectal cancer: a step forward in predicting prognosis and treatment response. Pharmocogenomics 9:1903-16, 2008 
84 $\mid$ Chapter 4

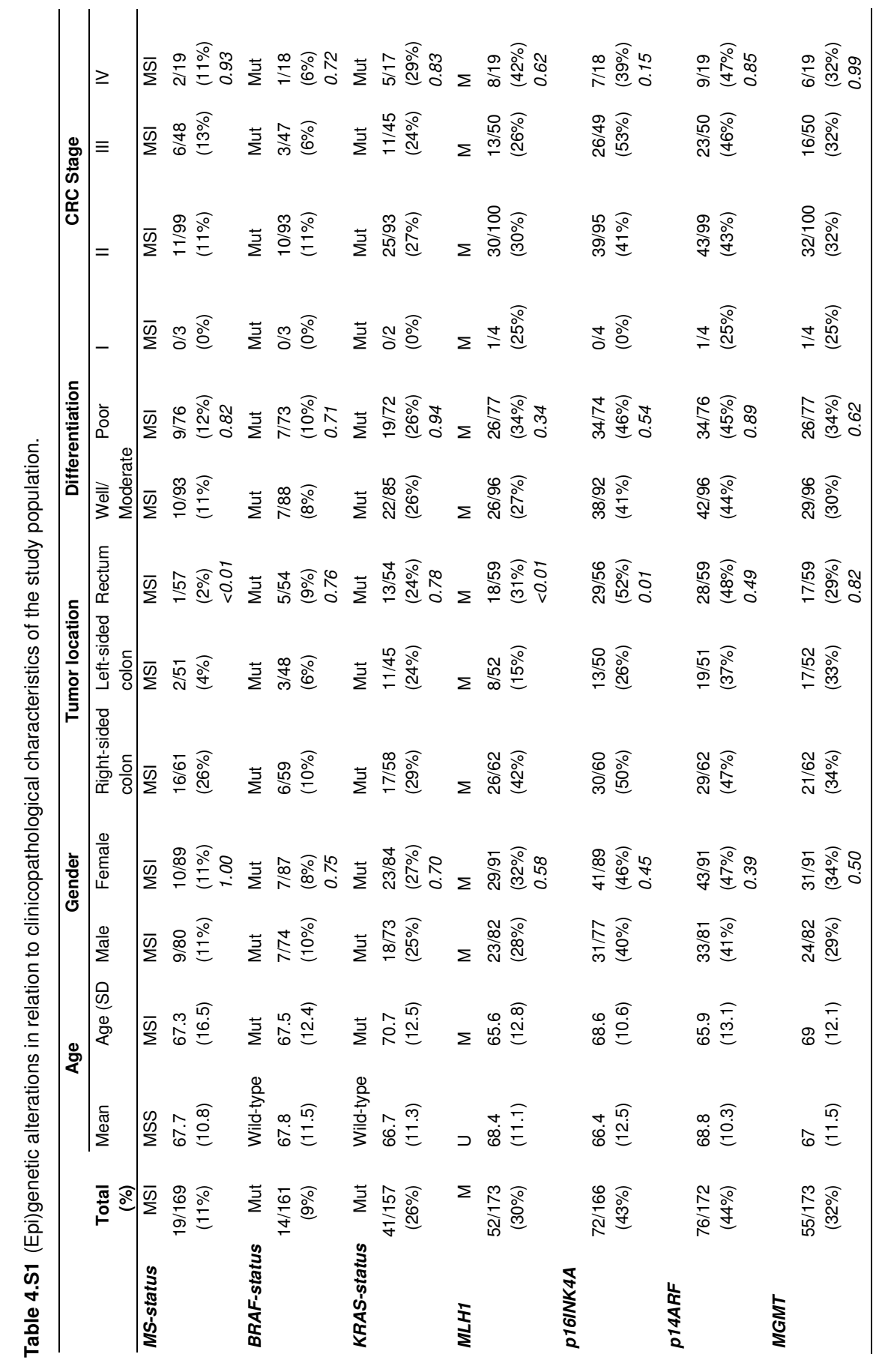




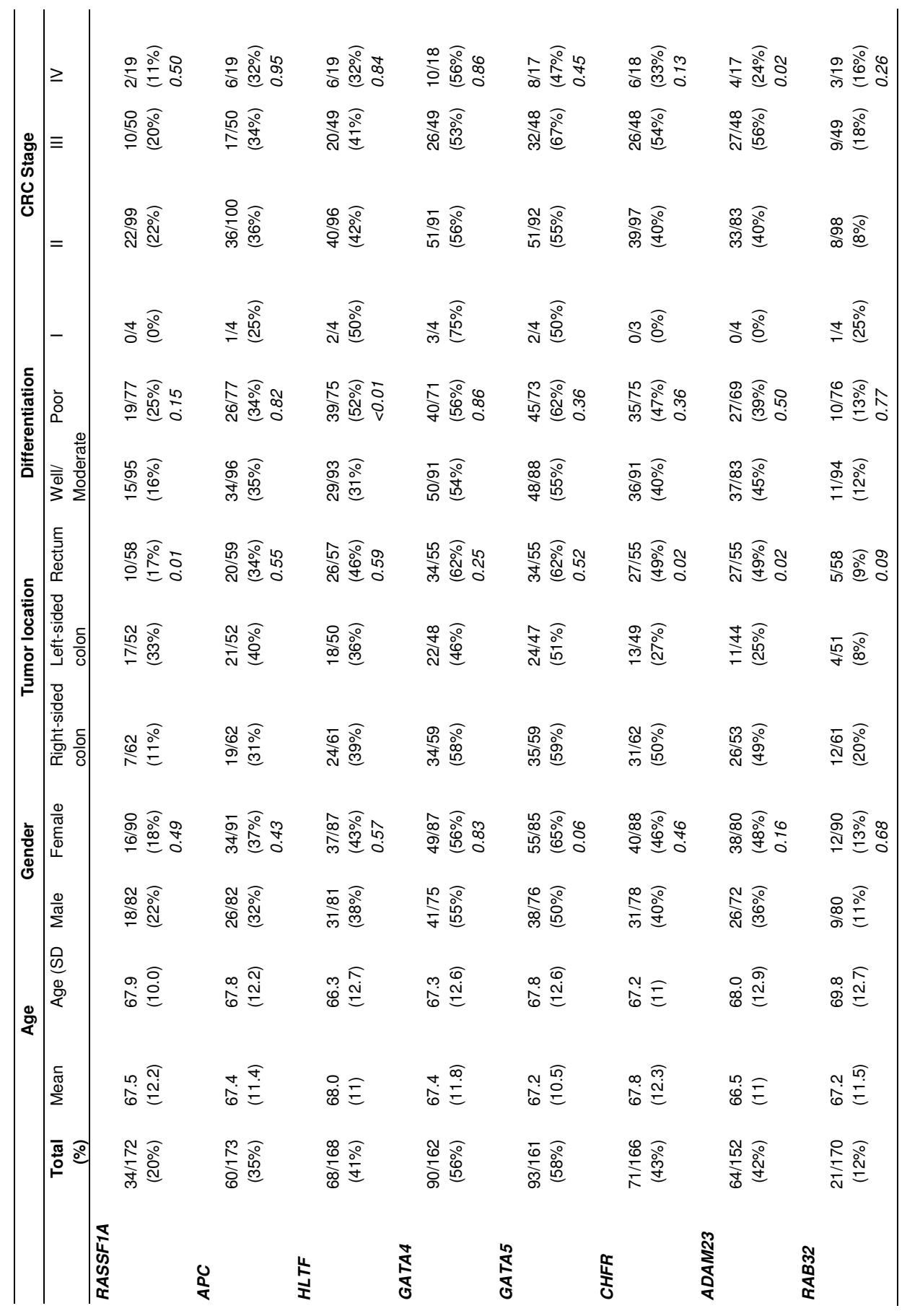


86 $\mid$ Chapter 4

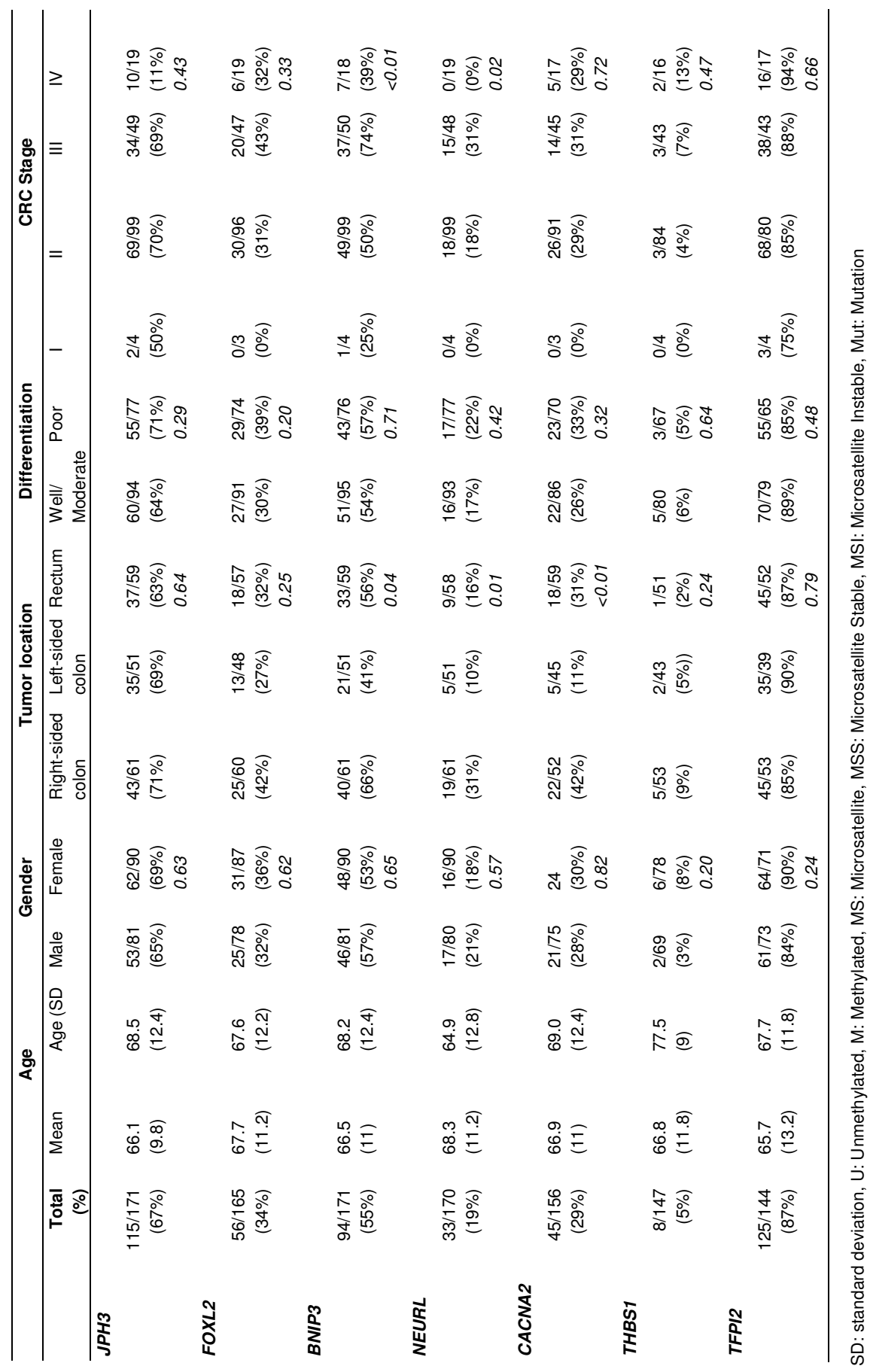


Promoter CpG island methylation and prognosis in colorectal cancer $\mid 87$

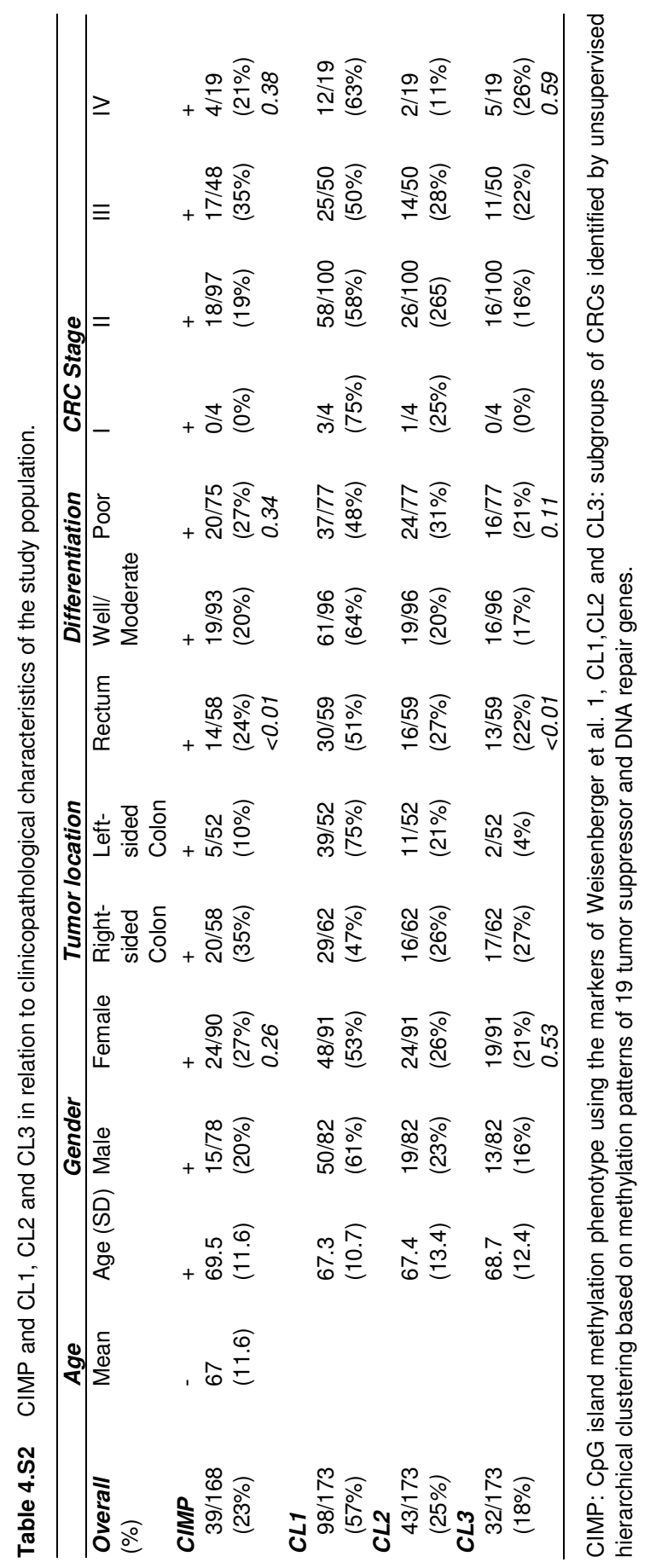


88 $\mid$ Chapter 4

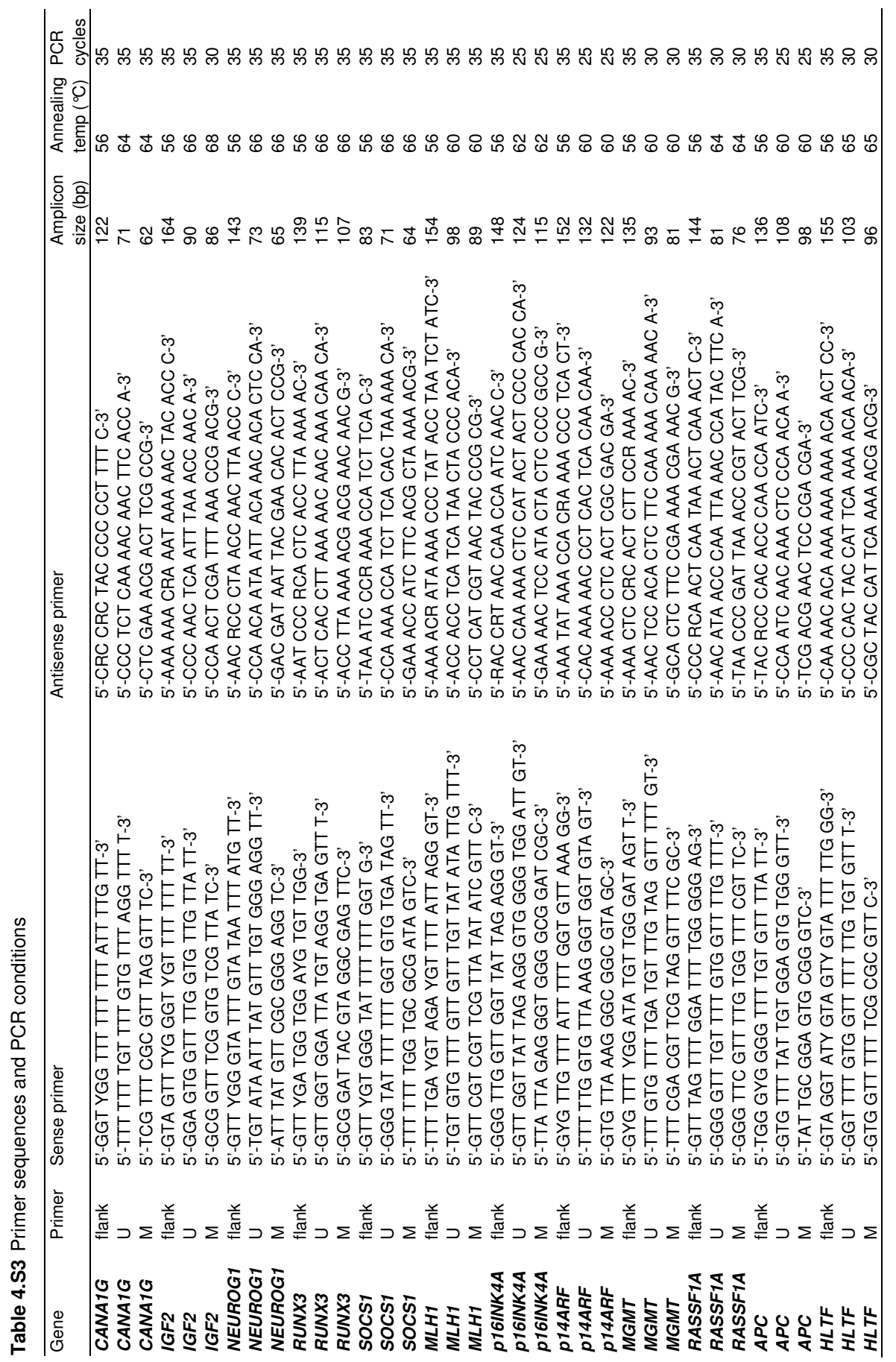


Promoter CpG island methylation and prognosis in colorectal cancer $\quad 89$

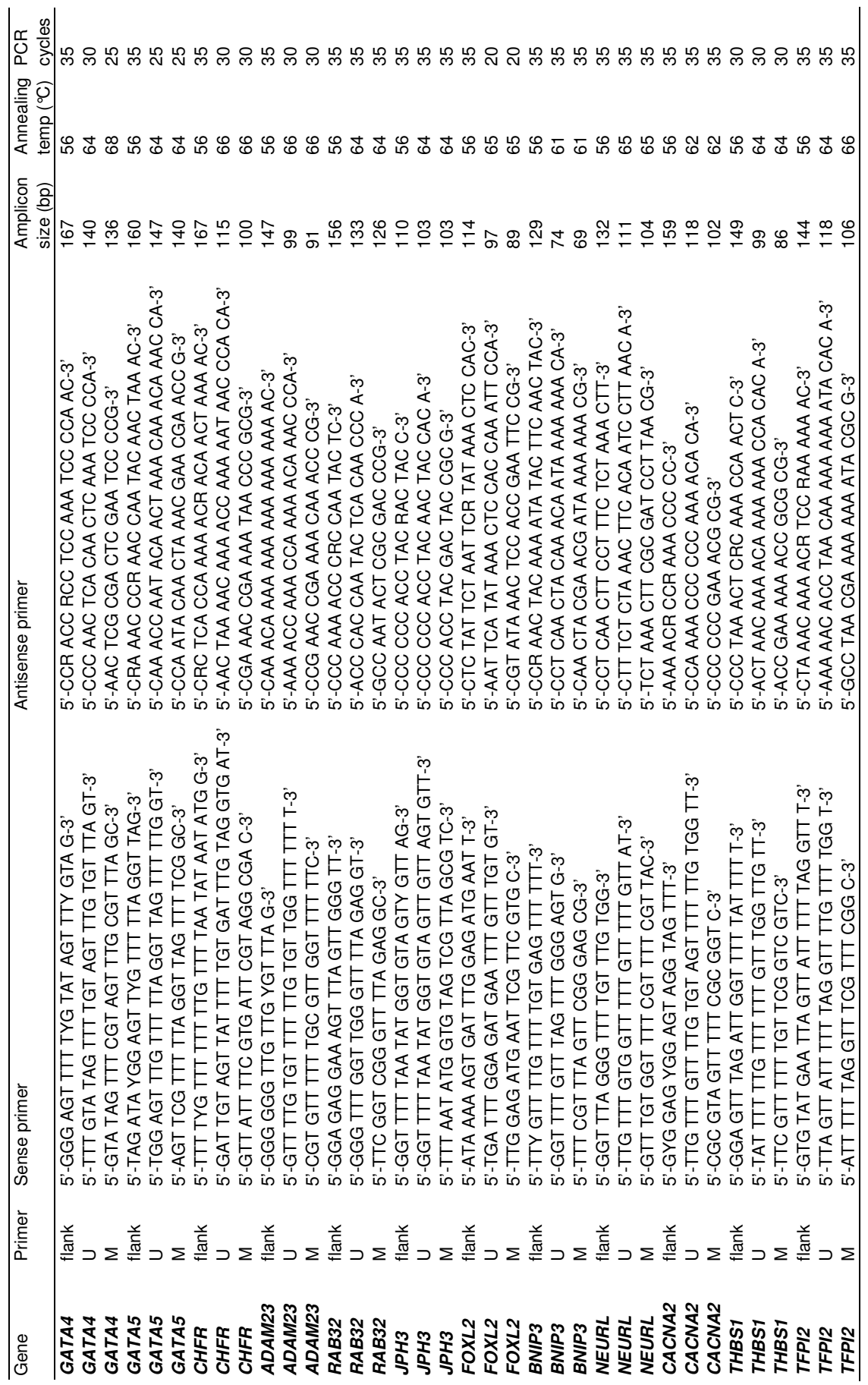


90 $\mid$ Chapter 4

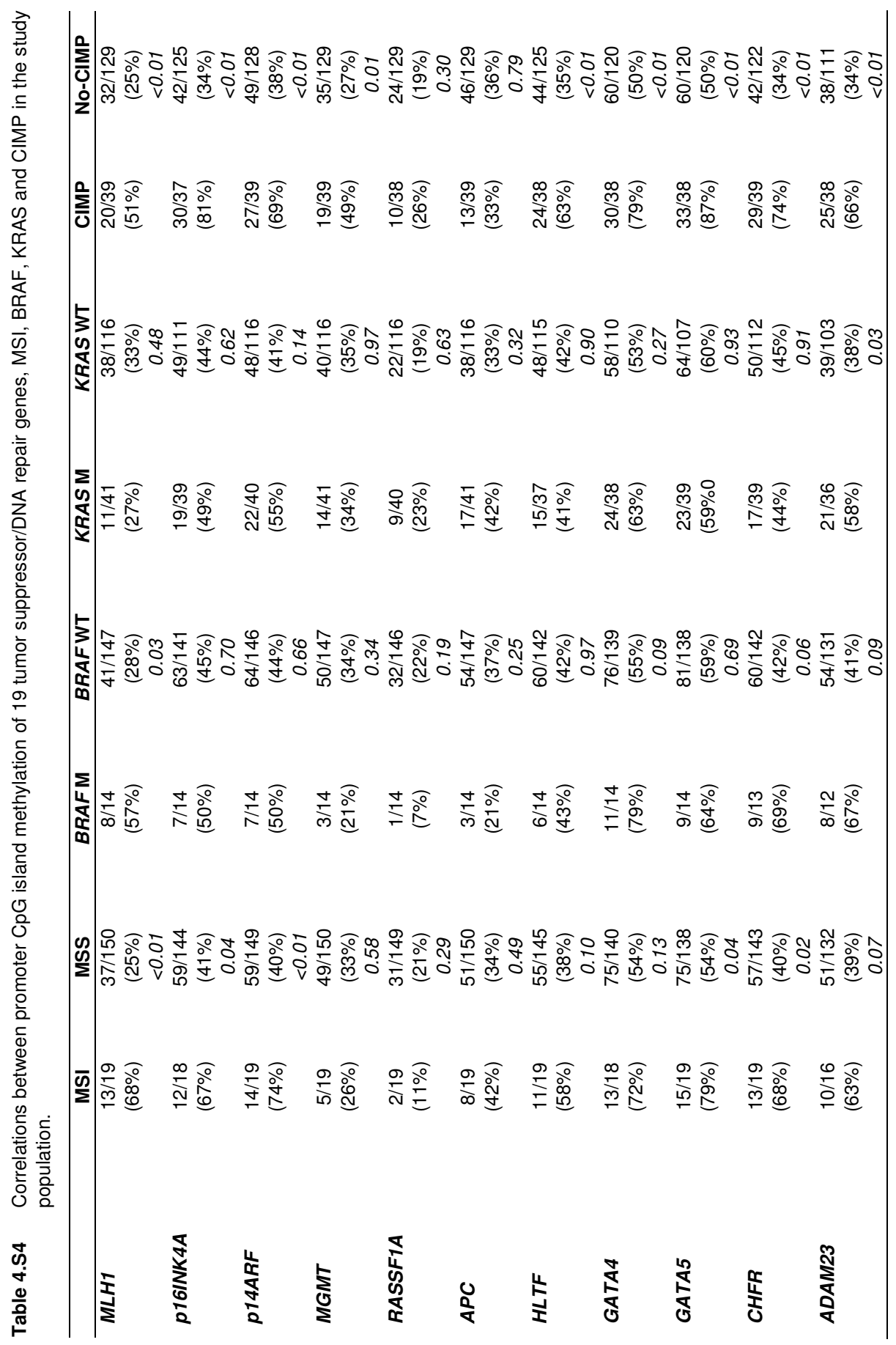


Promoter CpG island methylation and prognosis in colorectal cancer $\mid 91$

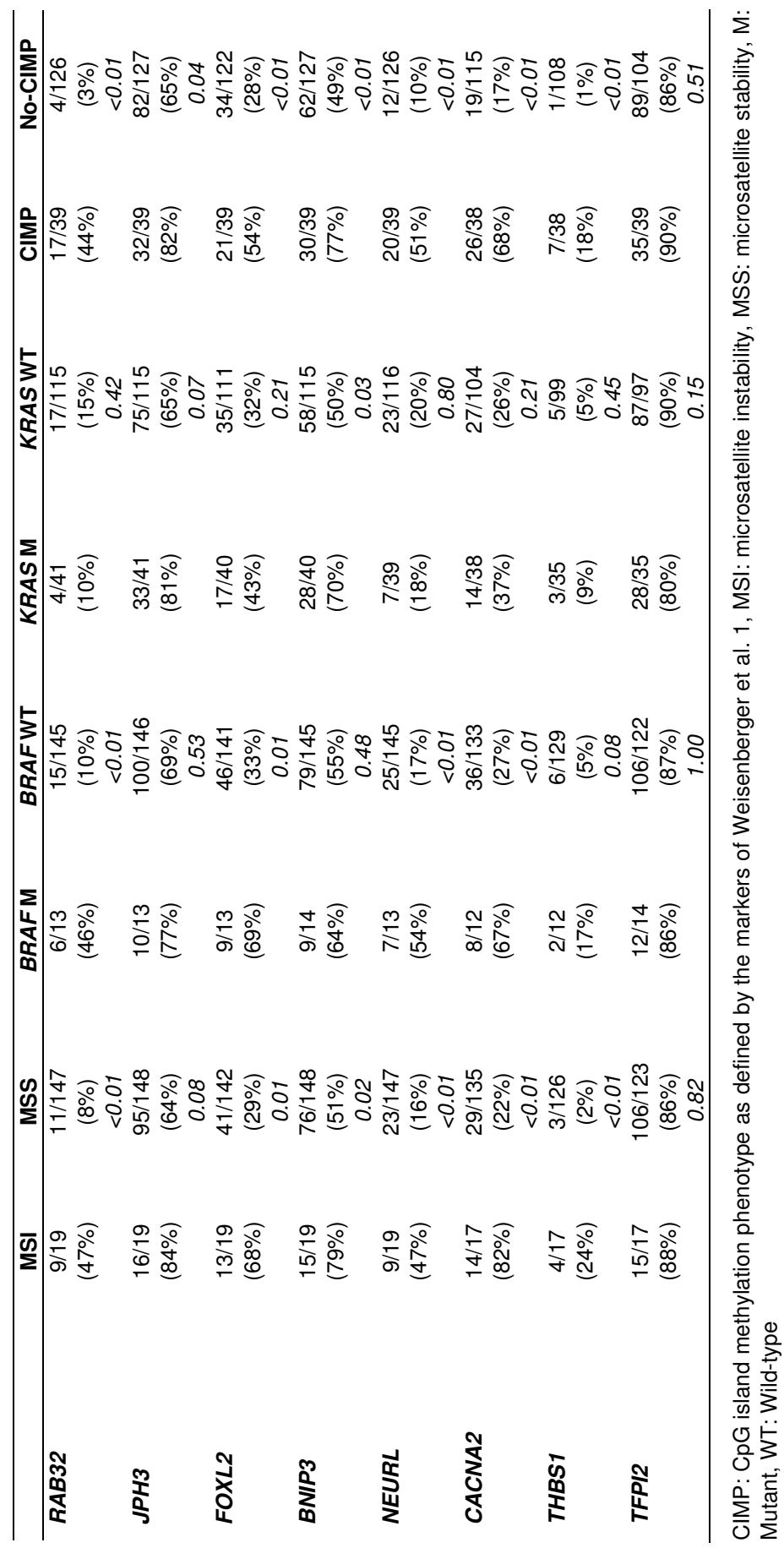


$92 \mid$ Chapter 4

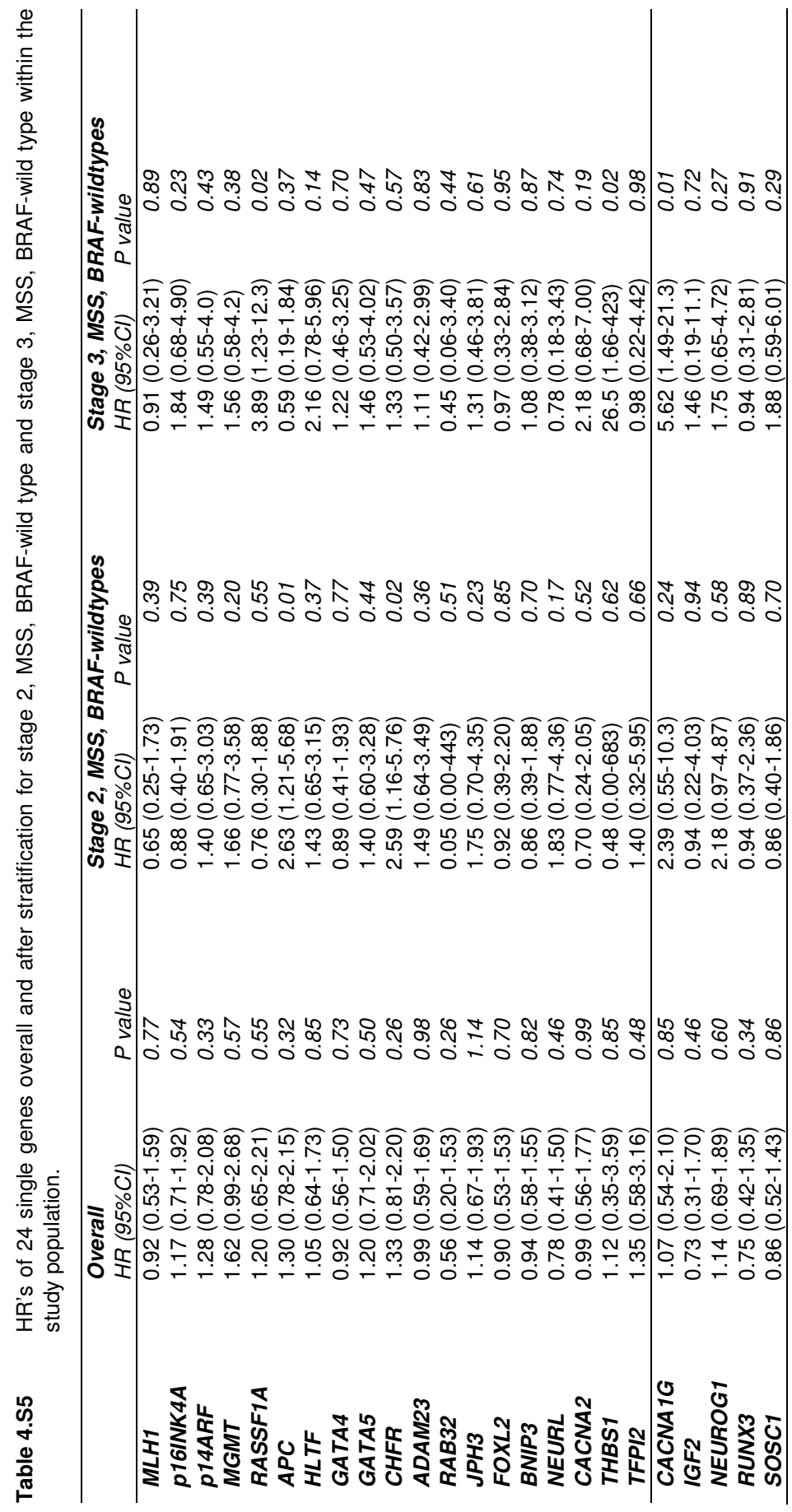




\title{
Chapter
}

\section{Emerging evidence for CHFR} as a cancer biomarker: update and prospects

\author{
S Derks \\ AHG Cleven \\ KM Smits \\ $W$ van Criekinge \\ AP de Bruïne \\ JG Herman \\ $M$ van Engeland
}




\section{Introduction}

Novel insights in the biology of cancers have revealed a variety of promising biomarkers for early detection of cancer and for predicting prognosis and response to therapy. Despite these insights and a revolution in biotechnology, only few biomarkers are implemented in current daily practice. Examples of operational biomarkers are testing for KRAS mutation and expression of EGFR to predict response to monoclonal antibodies against EGFR in lung ${ }^{1}$ and metastatic colon cancer ${ }^{2}$. However, EGFR antibodies are most frequently used as second or third line therapy and the number of patients that might benefit from this treatment is small. The great majority of patients still receive standard first line therapy. For this reason the use of chemotherapeutic agents is still very inefficient and too many patients experience unneeded toxicity.

In this review, we raise attention to a biomarker for which multiple lines of evidence are emerging: checkpoint with FHA and ring finger (CHFR). CHFR is a mitotic checkpoint- and tumor suppressor gene and is inactivated in a diversity of solid malignancies. Here we summarize the current evidence of altered CHFR expression in cancer and discuss its promising role as a prognostic and predictive biomarker.

\section{CHFR: an important regulator of cell cycle progression}

One of the hallmarks of cancer is loss of chromosomal instability, mostly due to dysfunction of one or more cell cycle checkpoints. The spindle assembly checkpoint is a key player in controlling chromosomal integrity by sensing microtubule kinetochore attachment and allowing promotion of mitosis only when chromosomes are attached properly.

Checkpoint with FHA and RING finger domain, CHFR, is an early mitotic checkpoint gene, which is more frequently inactivated in cancer than all mitotic checkpoint control genes together ${ }^{3}$. In case of microtubule- and radiation damaging stress CHFR is able to reversibly delay passage into mitosis before chromosome condensation has taken place ${ }^{4,5}$. CHFR localizes in the nucleus and becomes phosphorylated by protein kinase $B$ (PBK/AKT), a member of the PI3K signaling pathway ${ }^{6}$. The nuclear distribution, mobility and function of CHFR is modulated by interaction with promyelocytic leukemia protein (PML) bodies $^{7,8}$. PML bodies are involved in various cellular processes such as cellular senescence, apoptosis and maintenance of chromosomal stability and also the function of CHFR is dependent upon the interaction with PML bodies ${ }^{7}$. CHFR is an E3 ubiquitinin ligase which contains a FHA domain and a RING finger domain (Figure 5.1). The function of the FHA domain is largely unknown but is thought to mediate binding to phosphorylated proteins ${ }^{9}$ and is responsible for the anti-proliferative effect of CHFR. The RING finger domain is 
Emerging evidence for CHFR as a cancer biomarker $\mid 95$

able to ubiquitinate substrates such as polo-like kinase 1 (PLK1), Aurora A, MAD2 and CHFR itself ${ }^{9}$. By degradation of PLK1 CHFR inhibits the formation of the cyclin B1-Cdk kinase complex, which will result in a cell cycle arrest ${ }^{10}$. While evidence for degradation of PLK1 by CHFR in vivo is weak, ubiquitination of pro-mitotic protein Aurora $A$ and thereby maintaining chromosomal stability is clearly shown in vitro and in vivo ${ }^{11}$. The same accounts for ubiquitination of MAD2, which enables CHFR to control proper spindle attachment to kinetochores and thereby delays mitotic progression ${ }^{12,13,14}$. The interaction between CHFR and Aurora A and MAD2 is accomplished by the cysteine rich domain of $\mathrm{CHFR}^{11,12,15}$. Inside this cysteine rich region a RAR-binding zinc-finger (PBZ) is situated which is able to bind poly-(ADP-ribose)polymerases, important for checkpoint regulation and DNA damage response ${ }^{16}$.

More recently it was shown that CHFR also interacts with histone deacetylase HDAC $1^{17}$. HDAC1 is able to repress p21 and by inhibiting the deacetylase activity of HDAC1 CHFR restores the p21-G1 checkpoint ${ }^{17-19}$.

A

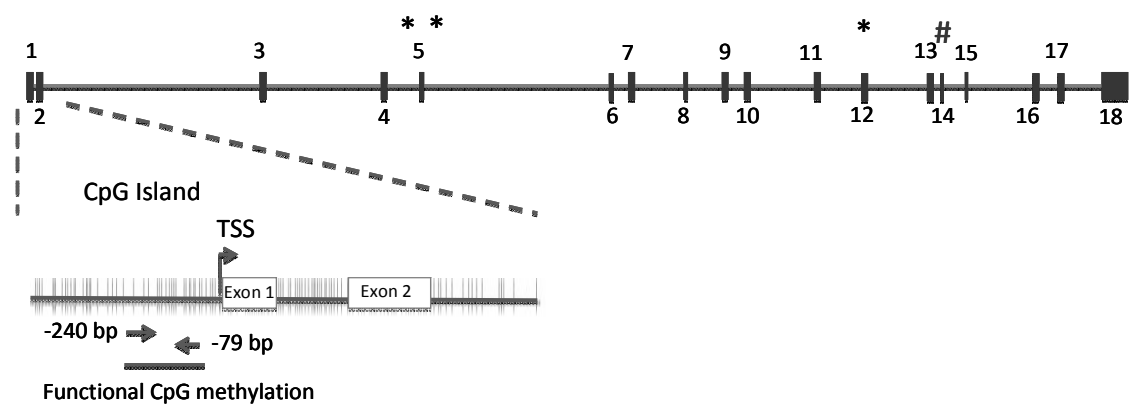

B

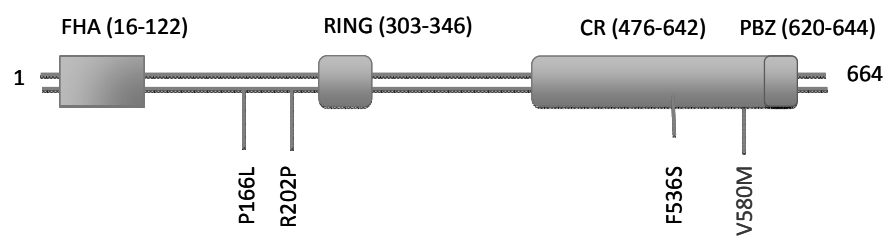

Figure 5.1 CHFR gene and protein. Schematic representation of promoter CpG island methylation, mutation and polymorphisms with functional significance of:

A) CHFR gene encompassing 18 exons. $\mathrm{CpG}$ island is enlarged with $\mathrm{CpG}$ dinucleotides as vertical lines. TSS: transcription start site * mutation. \# polymorphism. B) CHFR protein consisting of 664 aminoacids. FHA: forhead-associated domain. RING: ringfinger domain. CR: cysteine rich domain. PBZ: RAR-binding zinc-finger domain. Mutations in black, polymorphism in gray. 
$96 \mid$ Chapter 5

\section{CHFR is frequently inactivated in cancer}

Although many cancer cells have an impaired or unregulated spindle checkpoint, mutations of mitotic checkpoint genes are rare. Lack of expression of CHFR in cancer cells was first described by Scolnick et al. ${ }^{3}$ in one neuroblastoma and two colorectal cancer cell lines. Nonfunctional CHFR in these cell lines resulted in a high mitotic index when exposed to microtubule stress compared to wild-type cancer cells, which was restored by reintroduction of functional CHFR. A role for CHFR in tumor suppression became clear when CHFR deficient mice spontaneously developed malignant tumors ${ }^{11}$. CHFR knockout mice are viable and develop without defects but display invasive lymphomas and solid tumors (lung, liver, gastrointestinal) after 40 weeks. Furthermore, CHFR deficient mice show defects in chromosome segregation and aneuploidy. Also in a breast cancer cell line model decreased CHFR expression led to an accelerated growth rate, enhanced invasiveness, and amplified colony formation ${ }^{20}$. Since then, disrupted CHFR expression has been described in multiple cancer tissues (Table 5.1).

The tumor suppressor role of CHFR is not entirely clear. Due to its checkpoint function CHFR was thought to participate in the acquisition of chromosomal defects and a chromosomal instability phenotype. Although this was observed in previously described cell lines models, in primary colorectal- and breast cancer tissue this could not be observed ${ }^{25}$. To the contrary, CHFR inactivation was found to occur most frequently in cancers with microsatellite instability $(\mathrm{MSI})^{29}$ and $\mathrm{MLH} 1$ promoter CpG island methylation. Comparable results have been observed in gastric cancer ${ }^{55}$. The mechanism underlying the association between CHFR promoter methylation and $\mathrm{MSI}$ is unknown but might be explained the association with $M L H 1$ promoter methylation and a shared underlying DNA methylation defect. Future studies are needed to unravel the underlying mechanism, and a significant subset of MSS CRCs also has CHFR promoter methylation, which could be of clinical relevance.

Furthermore, a recent study shows an additional role for CHFR in regulating expression of pro-inflammatory chemokine interleukin $-8^{56}$. CHFR was able to inhibit the NFKB signaling pathway and IL-8, which subsequently resulted in decreased angiogenesis and cell migration ${ }^{57-59}$. Inactivation of $C H F R$ may trigger NFKB signaling activity, which results in increased angiogenesis, a metastatic phenotype and a poor prognosis. 


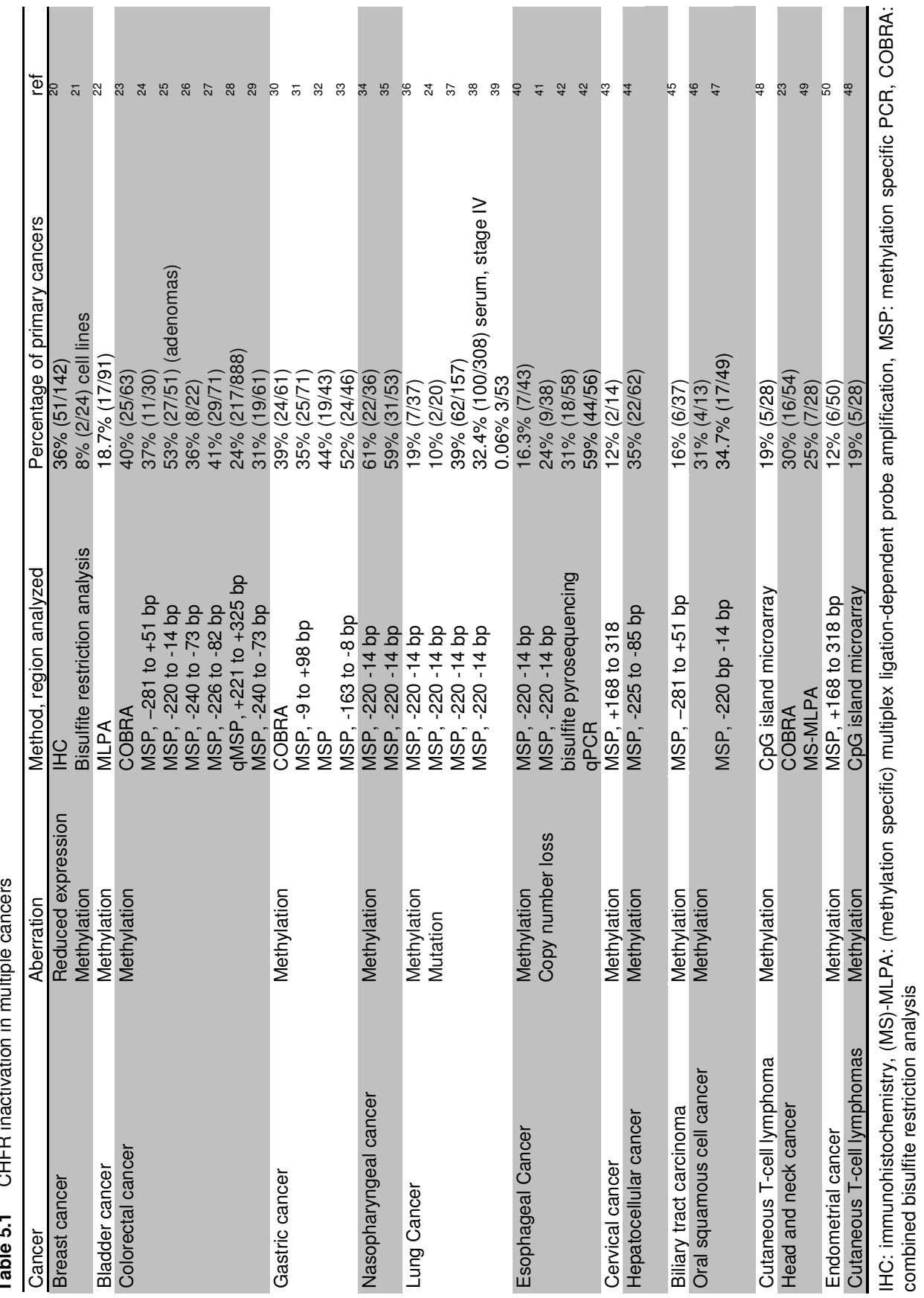


$98 \mid$ Chapter 5

\section{CHFR is inactivated by genetic and epigenetic mechanisms}

The original study of Scolnick and Halazonetis describes a sequence variation in the cysteine rich domain of CHFR in osteosarcoma cell line $\mathrm{U}_{20 S^{3}}$ (Figure 5.1). The variation consisted of a $G$ to $A$ transition leading to substitution of Val 539 with Met and was initially interpreted as potential missense mutation with functional impairment but turned out to be a functional polymorphism. ${ }^{36}$ The functional difference between wild-type and variant genotype was further studied in a series of 462 colorectal cancer patients and 245 healthy controls ${ }^{60}$. This study showed that the A allele of the GA variant was associated with a reduced colorectal cancer risk and absence of distant metastasis. Additional studies to identify structural variations in the CHFR coding sequence led to the identification of three missense mutations in non-small cell lung cancer (NSCLC) that all three were associated with a defective mitotic checkpoint ${ }^{39}$. Two mutations target the FHA en RING finger domain and the third is located in the cysteine rich region (Figure 5.1B). These mutations, however, were observed in only 3 out of 53 patients and are therefore rare events.

Loss of the chromosomal region harboring CHFR, 12q24.33, occurs more frequently. In esophageal adenocarcinomas, CHFR DNA copy number loss occurs in $59 \%(44 / 56)$ of esophageal cancers ${ }^{42}$ and is associated with reduced CHFR expression.

In most cancers, however, CHFR expression is reduced due to promoter $\mathrm{CpG}$ island methylation (Figure 5.1A). The promoter region of CHFR contains a CpG island spanning -905 bp to +783 bp relative to the transcription start site (using the lower limit values $500 \mathrm{bp}$ for length, $55 \%$ for GC content and 0.65 for ObsCpG/ExpCpG $\left.{ }^{61}\right)$. CHFR promoter methylation and subsequent transcriptional silencing was first described in esophageal cancer ${ }^{40}$, of which $16.3 \%(7 / 43)$ was hypermethylated while this was absent in adjacent normal tissues. Later it became clear that CHFR promoter methylation occurs in other cancers as well, among which colorectal-, gastric- and lung cancer (Table 5.1). Also in breast cancer CHFR becomes inactivated, however, only occasionally by promoter $\mathrm{CpG}$ island methylation ${ }^{20}$.

In most studies CHFR promoter CpG island methylation is assessed by methylation- specific PCR (MSP), which is an accurate and sensitive test to detect aberrant methylation in the primary tumor. In recent years is has become clear that the core region of $\mathrm{CpG}$ methylation within the $\mathrm{CpG}$ island which is biologically and clinically relevant can vary per gene ${ }^{62}$. For CHFR, CpG methylation within region -240 bp to -79 relative to the transcription start site is associated with reduced expression and clinical parameters such as prognosis $^{29}$ (Cleven et al, submitted) and therefore identified as core region. 
Emerging evidence for CHFR as a cancer biomarker $\mid 99$

\section{CHFR promoter methylation is associated with a poor prognosis and good response to microtubule inhibitors}

\section{CHFR promoter CpG island methylation as prognostic marker}

In recent years it has become increasingly apparent that CHFR promoter CpG island methylation is associated with a poor prognosis in multiple cancer types. For NSCLC two independent groups published the association between CHFR promoter $\mathrm{CpG}$ island methylation and an increased risk for recurrence and poor survival $^{38,51}$. Also for colorectal cancer a small retrospective study $(n=82)$ showed an association between CHFR promoter methylation and an increased risk of local recurrence as well as poor prognosis in stage III CRC ${ }^{52}$. We recently showed in a larger series of 173 patients that CHFR promoter methylation is an indicator of poor survival in stage II microsatellite stable CRC $(n=82)$ and validated these results in an independent prospective cohort study $(n=151)$ (Cleven et al, submitted).

Furthermore, different studies have shown the ability to detect CHFR promoter methylation in circulating tumor DNA in patients with non-small cell lung cancer $(\mathrm{NSCLC})^{38}$ and in peritoneal lavage in case of peritoneal metastasis in patients with gastric cancer ${ }^{31,32}$. Peritoneal micrometastases in gastric cancer are associated with a poor prognosis but difficult to detect. Testing for CHFR promoter methylation in DNA from peritoneal fluid can help to detect ${ }^{63,64}$ malignant cells and select patients that might benefit from additional chemotherapy.

Together these studies underscore the potential of CHFR promoter CpG island methylation as a prognostic marker.

\section{CHFR promoter CpG island methylation as predictor of response to therapy}

In addition to being a biomarker for poor prognosis in CRC and NSCLC, CHFR promoter $\mathrm{CpG}$ island methylation has been shown to be a potential predictor of response to microtubules inhibitors (Table 5.2).

Microtubule inhibitors such as docetaxel and paclitaxel disrupt normal microtubule dynamics during cell division by binding to the beta-tubulin subunits. This will lead to a failure of microtubule separation and apoptosis. CHFR is able to block entry into prophase until chromosomal alignment is restored, which will lead to decreased effect of taxanes. Cells expressing CHFR have shown to be more viable upon treatment with microtubule inhibitors compared to cells not expressing $C H F R^{65}$.

The association between CHFR expression and a poor sensitivity to microtubule inhibitors was first shown by Satoh et al. in gastric cancer cell lines ${ }^{30}$. Docetaxel is the second line therapy for gastric cancer; some patients 
respond while others are resistant. Therefore, CHFR promoter CpG island methylation was hypothesized to be an important determinant of response to therapy. Different studies to test this hypothesis have been performed with conflicting results. A small study restricted to twelve patients, showed that cancers with CHFR promoter $\mathrm{CpG}$ island methylation had a better clinical response compared to cancers with unmethylated $C H F R$, of which the majority showed progressive disease ${ }^{33}$. In a larger study $(n=41)$, no clear relationship between CHFR promoter $\mathrm{CpG}$ island methylation and response to microtubule inhibitors was observed ${ }^{66}$. Clinical response, however, was measured in metastatic lesions of which the methylation status of $C H F R$ was not assessed.

Since then, a potential role for CHFR promoter $\mathrm{CpG}$ island methylation in predicting response to microtubule inhibitors has been described in other cancer types as well. In NSCLC, CHFR promoter CpG island methylation is associated with high sensitivity to paclitaxel ${ }^{53}$. Interestingly, sensitivity to paclitaxel is correlated with wild-type EGFR and smoking habit, and the combination of the three is a strong predictor of clinical response to paclitaxel in NSCLC ${ }^{51,53}$.

Table 5.2 CHFR as marker of prognosis and response to microtubule inhibitor.

\section{Cancer}

\section{Prognostic markers}

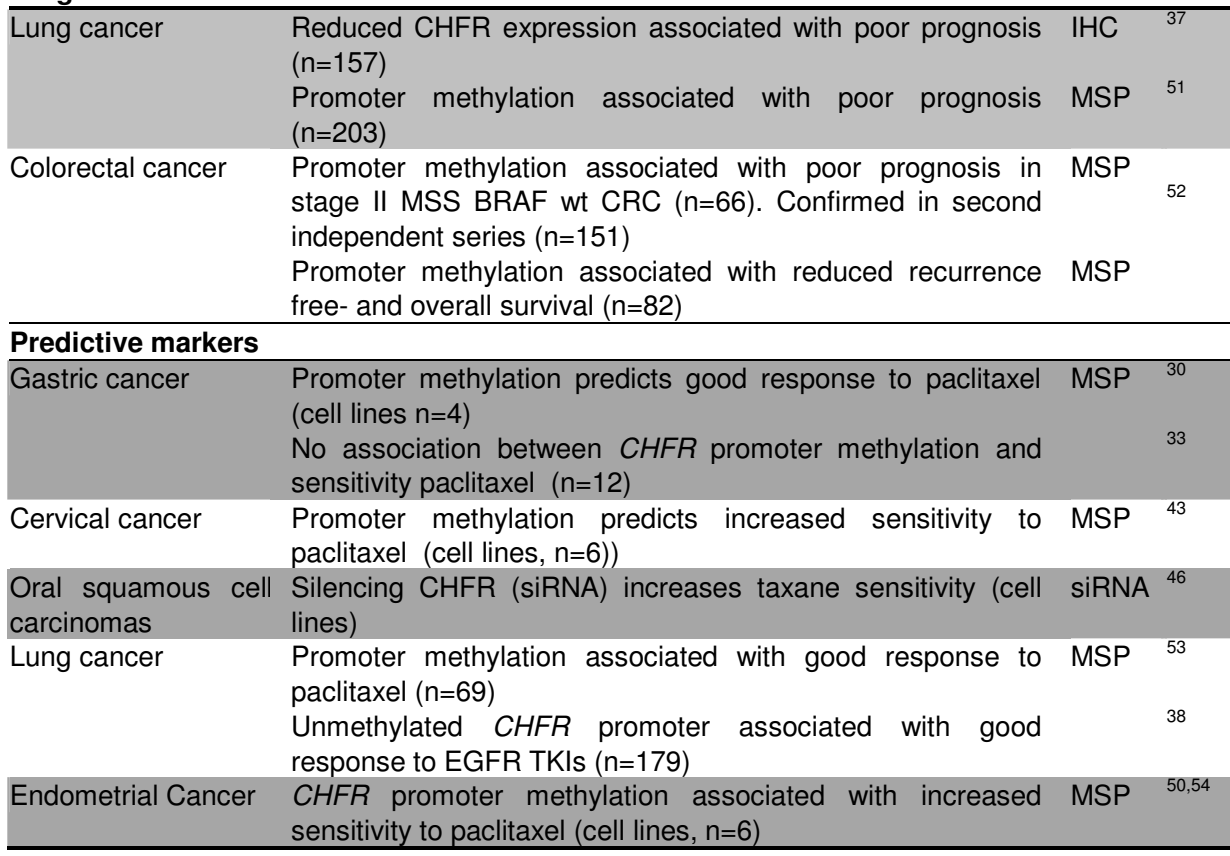

IHC: immunohistochemistry, MSP: methylation specific PCR, siRNA: short interference RNA 
Also in cervical adenocarcinomas, CHFR promoter $\mathrm{CpG}$ island methylation is proposed as biomarker to predict response to paclitaxel. In cervical adenocarcinomas, CHFR promoter CpG island methylation occurs in $14 \%$ of cases, in sharp contrast to cervical squamous cell carcinomas in which $C H F R$ is unmethylated ${ }^{67}$. Cervical adenocarcinomas respond to paclitaxel in $31 \%$ of cases, which is only $17 \%$ for other cervical cancers. Although this difference might involve CHFR promoter methylation, this relationship has only been shown in cervical cancer cell lines ${ }^{43}$. The same accounts for CHFR promoter methylation in endometrial cancers treated with paclitaxel, where CHFR promoter methylation is associated with increased sensitivity to paclitaxel, but is only tested in cell lines ${ }^{50,54}$. These results emphasize the need for bigger and better controlled patient series to investigate the role for CHFR promoter methylation as marker of response to taxanes.

\section{Conclusion}

In the last decade many studies have been performed to investigate CHFR promoter $\mathrm{CpG}$ island methylation as biomarker to predict prognosis and response to microtubule inhibitors in a diversity of cancers. There is compelling evidence that CHFR promoter methylation is a promising biomarker that can improve the management of multiple tumor types. Large, independent cohort studies and clinical trials to validate the prognostic and predictive value of CHFR promoter methylation are required and must be compared to established clinical markers such as for example the Tumor-Node-Metastasis (TNM) classification system. Simon et al. have proposed a less time consuming design in which archival material of prospective trials is used to investigate the performance of a single biomarker ${ }^{68}$.

Still the majority of candidate-biomarkers reported in literature do not reach clinical use, mostly because they fail to pass the validation phase. This can be explained by heterogeneity of the tumors and a technical inability to consistently verify the presence of the biomarker in patient material and the lack of specificity for a particular disease ${ }^{9}$. Furthermore, comparison between studies is difficult because different methylation detection methods are used and different regions within the $\mathrm{CpG}$ island are analyzed. It is therefore that a standardized method to assess CHFR promoter CpG methylation within the core region of the $\mathrm{CpG}$ island is needed. MSP is a very accurate, easy to handle method that can be used in the majority of laboratories and therefore a good method of choice. Especially since the core region of methylation which is associated with expression and prognosis has been identified and can also be detected by MBD2-affinity sequencing (unpublished results).

Although work is still in progress, currently available results all point towards the same direction, making CHFR promoter CpG island methylation a 
$102 \mid$ Chapter 5

biomarker with great potential and the development of clinical trials to study its predictive and prognostic power a priority. 
Emerging evidence for CHFR as a cancer biomarker $\mid 103$

\section{References}

1. Janku F, Garrido-Laguna I, Petruzelka LB, et al: Novel therapeutic targets in non-small cell lung cancer. Journal of thoracic oncology : official publication of the International Association for the Study of Lung Cancer 6:1601-12, 2011

2. Karapetis CS, Khambata-Ford S, Jonker DJ, et al: K-ras mutations and benefit from cetuximab in advanced colorectal cancer. The New England journal of medicine 359:1757-65, 2008

3. Scolnick DM, Halazonetis TD: Chfr defines a mitotic stress checkpoint that delays entry into metaphase. Nature 406:430-5, 2000

4. $\quad$ Durocher D, Jackson SP: The FHA domain. FEBS letters 513:58-66, 2002

5. Kang D, Chen J, Wong J, et al: The checkpoint protein Chfr is a ligase that ubiquitinates Plk1 and inhibits Cdc2 at the G2 to M transition. The Journal of cell biology 156:249-59, 2002

6. Shtivelman E: Promotion of mitosis by activated protein kinase B after DNA damage involves polo-like kinase 1 and checkpoint protein CHFR. Molecular cancer research : MCR 1:959-69, 2003

7. Daniels MJ, Marson A, Venkitaraman AR: PML bodies control the nuclear dynamics and function of the CHFR mitotic checkpoint protein. Nature structural \& molecular biology 11:1114-21, 2004

8. Fukuda $\mathrm{T}$, Kondo $\mathrm{Y}$, Nakagama $\mathrm{H}$ : The anti-proliferative effects of the CHFR depend on the forkhead associated domain, but not E3 ligase activity mediated by ring finger domain. PloS one 3:e1776, 2008

9. Li J, Williams BL, Haire LF, et al: Structural and functional versatility of the FHA domain in DNA-damage signaling by the tumor suppressor kinase Chk2. Molecular cell 9:1045-54, 2002

10. Summers MK, Bothos J, Halazonetis TD: The CHFR mitotic checkpoint protein delays cell cycle progression by excluding Cyclin B1 from the nucleus. Oncogene 24:2589-98, 2005

11. Yu X, Minter-Dykhouse $\mathrm{K}$, Malureanu $\mathrm{L}$, et al: Chfr is required for tumor suppression and Aurora A regulation. Nature genetics 37:401-6, 2005

12. Privette LM, Weier JF, Nguyen HN, et al: Loss of CHFR in human mammary epithelial cells causes genomic instability by disrupting the mitotic spindle assembly checkpoint. Neoplasia 10:643-52, 2008

13. Keller JA, Petty EM: CHFR binds to and regulates MAD2 in the spindle checkpoint through its cysteine-rich domain. Biochemical and biophysical research communications 409:389-93, 2011

14. Fang $\mathrm{G}, \mathrm{Yu} \mathrm{H}$, Kirschner MW: The checkpoint protein MAD2 and the mitotic regulator CDC20 form a ternary complex with the anaphase-promoting complex to control anaphase initiation. Genes \& development 12:1871-83, 1998

15. Vader G, Lens SM: The Aurora kinase family in cell division and cancer. Biochimica et biophysica acta 1786:60-72, 2008

16. Ahel I, Ahel D, Matsusaka $T$, et al: Poly(ADP-ribose)-binding zinc finger motifs in DNA repair/checkpoint proteins. Nature 451:81-5, 2008

17. Oh YM, Kwon YE, Kim JM, et al: Chfr is linked to tumour metastasis through the downregulation of HDAC1. Nature cell biology 11:295-302, 2009

18. Vidal A, Koff A: Cell-cycle inhibitors: three families united by a common cause. Gene 247:115,2000

19. Kim JH, Kim B, Cai L, et al: Transcriptional regulation of a metastasis suppressor gene by Tip60 and beta-catenin complexes. Nature 434:921-6, 2005

20. Privette LM, Gonzalez ME, Ding L, et al: Altered expression of the early mitotic checkpoint protein, CHFR, in breast cancers: implications for tumor suppression. Cancer research 67:6064-74, 2007

21. Erson AE, Petty EM: CHFR-associated early G2/M checkpoint defects in breast cancer cells. Molecular carcinogenesis 39:26-33, 2004 
$104 \mid$ Chapter 5

22. Agundez M, Grau L, Palou J, et al: Evaluation of the Methylation Status of Tumour Suppressor Genes for Predicting Bacillus Calmette-Guerin Response in Patients With T1G3 High-Risk Bladder Tumours. European urology 60:131-40, 2011

23. Toyota $M$, Sasaki $Y$, Satoh $A$, et al: Epigenetic inactivation of CHFR in human tumors. Proceedings of the National Academy of Sciences of the United States of America 100:781823, 2003

24. Corn PG, Summers MK, Fogt $F$, et al: Frequent hypermethylation of the 5 ' $\mathrm{CpG}$ island of the mitotic stress checkpoint gene Chfr in colorectal and non-small cell lung cancer. Carcinogenesis 24:47-51, 2003

25. Bertholon J, Wang Q, Falette $\mathrm{N}$, et al: Chfr inactivation is not associated to chromosomal instability in colon cancers. Oncogene 22:8956-60, 2003

26. Derks S, Postma C, Carvalho B, et al: Integrated analysis of chromosomal, microsatellite and epigenetic instability in colorectal cancer identifies specific associations between promoter methylation of pivotal tumour suppressor and DNA repair genes and specific chromosomal alterations. Carcinogenesis 29:434-9, 2008

27. Morioka $Y$, Hibi K, Sakai M, et al: Aberrant methylation of the CHFR gene is frequently detected in non-invasive colorectal cancer. Anticancer research 26:4267-70, 2006

28. Kawasaki T, Ohnishi M, Nosho K, et al: CpG island methylator phenotype-low (CIMP-low) colorectal cancer shows not only few methylated CIMP-high-specific CpG islands, but also low-level methylation at individual loci. Modern pathology : an official journal of the United States and Canadian Academy of Pathology, Inc 21:245-55, 2008

29. Brandes JC, van Engeland M, Wouters KA, et al: CHFR promoter hypermethylation in colon cancer correlates with the microsatellite instability phenotype. Carcinogenesis 26:1152-6, 2005

30. Satoh A, Toyota M, Itoh F, et al: Epigenetic inactivation of CHFR and sensitivity to microtubule inhibitors in gastric cancer. Cancer research 63:8606-13, 2003

31. Honda T, Tamura G, Waki T, et al: Promoter hypermethylation of the Chfr gene in neoplastic and non-neoplastic gastric epithelia. British journal of cancer 90:2013-6, 2004

32. Kang HC, Kim IJ, Park JH, et al: Promoter hypermethylation and silencing of CHFR mitotic stress checkpoint gene in human gastric cancers. Oncology reports 12:129-33, 2004

33. Koga $Y$, Kitajima $\mathrm{Y}$, Miyoshi A, et al: The significance of aberrant CHFR methylation for clinical response to microtubule inhibitors in gastric cancer. Journal of gastroenterology 41:133-9, 2006

34. Cheung HW, Ching YP, Nicholls JM, et al: Epigenetic inactivation of CHFR in nasopharyngeal carcinoma through promoter methylation. Molecular carcinogenesis 43:23745, 2005

35. Hutajulu SH, Indrasari SR, Indrawati LP, et al: Epigenetic markers for early detection of nasopharyngeal carcinoma in a high risk population. Molecular cancer 10:48, 2011

36. Mizuno $\mathrm{K}$, Osada $\mathrm{H}$, Konishi $\mathrm{H}$, et al: Aberrant hypermethylation of the CHFR prophase checkpoint gene in human lung cancers. Oncogene 21:2328-33, 2002

37. Takeshita M, Koga T, Takayama K, et al: CHFR expression is preferentially impaired in smoking-related squamous cell carcinoma of the lung, and the diminished expression significantly harms outcomes. International journal of cancer. Journal international du cancer 123:1623-30, 2008

38. Salazar F, Molina MA, Sanchez-Ronco M, et al: First-line therapy and methylation status of CHFR in serum influence outcome to chemotherapy versus EGFR tyrosine kinase inhibitors as second-line therapy in stage IV non-small-cell lung cancer patients. Lung cancer 72:84-91, 2011

39. Mariatos $\mathrm{G}$, Bothos $\mathrm{J}$, Zacharatos $\mathrm{P}$, et al: Inactivating mutations targeting the chfr mitotic checkpoint gene in human lung cancer. Cancer research 63:7185-9, 2003

40. Shibata $\mathrm{Y}$, Haruki N, Kuwabara $\mathrm{Y}$, et al: Chfr expression is downregulated by $\mathrm{CpG}$ island hypermethylation in esophageal cancer. Carcinogenesis 23:1695-9, 2002

41. Morioka Y, Hibi K, Sakai M, et al: Aberrant methylation of the CHFR gene in digestive tract cancer. Anticancer research 26:1791-5, 2006 
Emerging evidence for CHFR as a cancer biomarker $\mid 105$

42. Soutto M, Peng D, Razvi M, et al: Epigenetic and genetic silencing of CHFR in esophageal adenocarcinomas. Cancer 116:4033-42, 2010

43. Banno K, Yanokura M, Kawaguchi M, et al: Epigenetic inactivation of the CHFR gene in cervical cancer contributes to sensitivity to taxanes. International journal of oncology 31:71320, 2007

44. Sakai M, Hibi K, Kanazumi N, et al: Aberrant methylation of the CHFR gene in advanced hepatocellular carcinoma. Hepato-gastroenterology 52:1854-7, 2005

45. Tozawa T, Tamura G, Honda T, et al: Promoter hypermethylation of DAP-kinase is associated with poor survival in primary biliary tract carcinoma patients. Cancer science 95:736-40, 2004

46. Ogi $\mathrm{K}$, Toyota M, Mita $\mathrm{H}$, et al: Small interfering RNA-induced CHFR silencing sensitizes oral squamous cell cancer cells to microtubule inhibitors. Cancer biology \& therapy 4:773-80, 2005

47. Baba S, Hara A, Kato K, et al: Aberrant promoter hypermethylation of the CHFR gene in oral squamous cell carcinomas. Oncology reports 22:1173-9, 2009

48. van Doorn R, Zoutman WH, Dijkman R, et al: Epigenetic profiling of cutaneous T-cell lymphoma: promoter hypermethylation of multiple tumor suppressor genes including BCL7a, PTPRG, and p73. Journal of clinical oncology : official journal of the American Society of Clinical Oncology 23:3886-96, 2005

49. Chen K, Sawhney R, Khan M, et al: Methylation of multiple genes as diagnostic and therapeutic markers in primary head and neck squamous cell carcinoma. Archives of otolaryngology--head \& neck surgery 133:1131-8, 2007

50. Yanokura M, Banno K, Kawaguchi M, et al: Relationship of aberrant DNA hypermethylation of CHFR with sensitivity to taxanes in endometrial cancer. Oncology reports 17:41-8, 2007

51. Koga T, Takeshita M, Yano T, et al: CHFR hypermethylation and EGFR mutation are mutually exclusive and exhibit contrastive clinical backgrounds and outcomes in non-small cell lung cancer. International journal of cancer. Journal international du cancer 128:1009-17, 2011

52. Tanaka M, Chang P, Li Y, et al: Association of CHFR Promoter Methylation with Disease Recurrence in Locally Advanced Colon Cancer. Clinical cancer research : an official journal of the American Association for Cancer Research 17:4531-40, 2011

53. Takeshita M, Koga T, Takayama K, et al: Alternative efficacy-predicting markers for paclitaxel instead of CHFR in non-small-cell lung cancer. Cancer biology \& therapy 10:933-41, 2010

54. Wang $\mathrm{X}$, Yang $\mathrm{Y}, \mathrm{Xu} \mathrm{C}$, et al: CHFR Suppression by Hypermethylation Sensitizes Endometrial Cancer Cells to Paclitaxel. International journal of gynecological cancer : official journal of the International Gynecological Cancer Society 21:996-1003, 2011

55. Homma N, Tamura G, Honda T, et al: Hypermethylation of Chfr and hMLH1 in gastric noninvasive and early invasive neoplasias. Virchows Archiv : an international journal of pathology 446:120-6, 2005

56. Kashima L, Toyota M, Mita H, et al: CHFR, a potential tumor suppressor, downregulates interleukin-8 through the inhibition of NF-kappaB. Oncogene 28:2643-53, 2009

57. Cuenca RE, Azizkhan RG, Haskill S: Characterization of GRO alpha, beta and gamma expression in human colonic tumours: potential significance of cytokine involvement. Surgical oncology $1: 323-9,1992$

58. Nurnberg W, Tobias D, Otto F, et al: Expression of interleukin-8 detected by in situ hybridization correlates with worse prognosis in primary cutaneous melanoma. The Journal of pathology 189:546-51, 1999

59. Lee $\mathrm{KH}$, Bae SH, Lee JL, et al: Relationship between urokinase-type plasminogen receptor, interleukin-8 gene expression and clinicopathological features in gastric cancer. Oncology 66:210-7, 2004

60. Kang HC, Kim IJ, Jang SG, et al: Coding region polymorphisms in the CHFR mitotic stress checkpoint gene are associated with colorectal cancer risk. Cancer letters 260:170-9, 2008

61. Gardiner-Garden M, Frommer M: CpG islands in vertebrate genomes. Journal of molecular biology 196:261-82, 1987 
62. van Vlodrop IJ, Niessen HE, Derks S, et al: Analysis of promoter CpG island hypermethylation in cancer: location, location, location! Clinical cancer research : an official journal of the American Association for Cancer Research 17:4225-31, 2011

63. Hiraki M, Kitajima Y, Sato S, et al: Aberrant gene methylation in the peritoneal fluid is a risk factor predicting peritoneal recurrence in gastric cancer. World journal of gastroenterology : WJG 16:330-8, 2010

64. Hiraki M, Kitajima $Y$, Koga $Y$, et al: Aberrant Gene Methylation Is a Biomarker for the Detection of Cancer Cells in Peritoneal Wash Samples from Advanced Gastric Cancer Patients. Annals of surgical oncology, 2011

65. Chaturvedi $\mathrm{P}$, Sudakin $\mathrm{V}$, Bobiak ML, et al: Chfr regulates a mitotic stress pathway through its RING-finger domain with ubiquitin ligase activity. Cancer research 62:1797-801, 2002

66. Yoshida K, Hamai Y, Suzuki T, et al: DNA methylation of CHFR is not a predictor of the response to docetaxel and paclitaxel in advanced and recurrent gastric cancer. Anticancer research 26:49-54, 2006

67. Curtin JP, Blessing JA, Webster KD, et al: Paclitaxel, an active agent in nonsquamous carcinomas of the uterine cervix: a Gynecologic Oncology Group Study. Journal of clinical oncology : official journal of the American Society of Clinical Oncology 19:1275-8, 2001

68. Simon RM, Paik S, Hayes DF: Use of archived specimens in evaluation of prognostic and predictive biomarkers. Journal of the National Cancer Institute 101:1446-52, 2009 


\section{Chapter}

Stromal expression of hypoxia regulated proteins is an adverse prognostic factor in colorectal carcinomas

AHG Cleven $M$ van Engeland BG Wouters AP de Bruïne 


\section{Abstract}

Hypoxia modifies the phenotype of tumors in a way that promotes tumor aggressiveness and resistance towards chemotherapy and radiotherapy. However, the expression and influence of hypoxia-regulated proteins on tumor biology are not well characterized in colorectal tumors. We

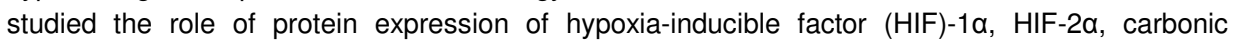
anhydrase 9 (CA9) and glucose transporter 1 (GLUT1) in patients with colorectal adenocarcinomas.

Expression of HIF-1a, HIF-2a, CA9 and GLUT1 was quantified by immunohistochemistry in 133 colorectal adenocarcinomas. The expression of hypoxia markers was correlated with clinicopathological variables and overall patient survival.

Expression of these hypoxia markers was detected in the epithelial compartment of the tumor cells as well as in tumor-associated stromal cells. Although tumor cells frequently showed expression of one or more of the investigated hypoxia markers, no correlation among these markers or with clinical response was found. However, within the tumor stroma, positive correlations between the hypoxia markers HIF-2a, CA9 and GLUT1 were observed. Furthermore expression of HIF-2 $\alpha$ and CA9 in tumor-associated stroma were both associated with a significantly reduced overall survival. In the Cox proportional hazard model, stromal HIF-2 $\alpha$ expression was an independent prognostic factor for survival.

These observations show, that expression of hypoxia regulated proteins in tumor-associated stromal cells, as opposed to their expression in epithelial tumor cells, is associated with poor outcome in colorectal cancer. This study suggests that tumor hypoxia may influence tumorassociated stromal cells in a way that ultimately contributes to patient prognosis. 


\section{Introduction}

Regulation of tissue oxygen homeostasis is critical for cell function, behavior and survival. Lack of oxygen (hypoxia), occurs early and remains a common feature of tumors throughout their development. Hypoxic areas in tumors contribute to a worse prognosis independent of treatment modality ${ }^{1,2}$. One of the underlying mechanisms proposed to account for this poor prognosis is a contribution of hypoxia to the malignant status of tumors through promotion of metastasis, angiogenesis, and selection of cells with defects in apoptosis ${ }^{3}$. In colorectal cancer, the importance of hypoxia has been demonstrated by clinical studies in which hypoxia predicts for worse outcome and resistance towards chemotherapy and radiotherapy $\mathrm{y}^{4,5}$.

The majority of studies investigating hypoxia in human tumors has been carried out with oxygen sensing needle electrodes that are capable of direct oxygen measurements in tumors ${ }^{6}$. However, this procedure is invasive and restricted to accessible tumor sites ${ }^{7}$. This has led to the development and testing of alternative hypoxia markers including the 2-nitroimidazole compounds pimonidazole and EF-5 which undergo reduction upon binding within hypoxic cells $^{8}$. Estimation of hypoxia by these so-called "exogenous" markers is made several hours following intravenous administration, by evaluating the degree of binding using specific antibodies in biopsies ${ }^{9}$. In addition, several "endogenous" markers have also been proposed. These markers consist of hypoxia regulated gene products and include among others hypoxia inducible factor (HIF)-1a, HIF-2a, carbonic anhydrase 9 (CA9), glucose transporter 1 (GLUT1), glucose transporter 3 (GLUT3), and vascular endothelial growth factor (VEGF) ${ }^{9-17}$.

$\mathrm{HIF}$ is a heterodimeric transcription factor composed of $\alpha$ and $\beta$ subunits. HIF$1 \beta$ is expressed constitutively and is not sensitive to the hypoxic status of the cells, whereas the alpha subunit accumulates rapidly inside the hypoxic cells primarily because of the prevention of ubiquitination and subsequent protein degradation by the proteasome complex, which usually takes place in normoxic cells. There are three homologues of the alpha subunit (HIF-1 $\alpha$, HIF-2 $\alpha$, HIF$3 \alpha)^{18}$. HIF-1 $\alpha$ and HIF-2 $\alpha$ are thought to play a significant role in tumor neovascularization, enhanced cellular proliferation, decreased apoptosis, and development of drug resistance to chemotherapeutic agents ${ }^{19}$. In colorectal cancer HIF-2 $\alpha$ also seems to play an important role in angiogenesis, the combined expression of HIF-2 $\alpha$ and HIF-1 $\alpha$ may play a role in tumor progression and prognosis ${ }^{10,20}$.

CA9 is a transmembrane glycoprotein and a member of the carbonic anhydrase family that is regulated by $\mathrm{HIF}^{21}$. Carbonic anhydrases are zinc metalloenzymes that catalyze the reversible conversion of carbon dioxide to carbonic acid and are involved in respiration, calcification, acid-base balance, the formation of cerebrospinal fluid, saliva, and gastric acid. Increased CA9 
expression is induced by hypoxia, as was shown in a wide spectrum of tumor types including tumors of the uterine cervix, head and neck, lung, bladder, breast, esophagus, colon and rectum ${ }^{22-29}$. CA9 expression has previously been demonstrated in colorectal tumors by Saarnio et al. (1998). CA9 staining intensity in colorectal tumors was higher in less advanced tumors (Dukes A + B) $)^{29,30}$.

GLUT1 is a member of the glucose transporter family of proteins facilitating independent transport of glucose that is also regulated by HIF. Tumors have accelerated metabolism and increased requirements for ATP production, therefore cancer cells have high rates of glycolysis. Elevated GLUT1 expression under hypoxic conditions has been described for many cancers, including hepatic, pancreatic, breast, esophageal, brain, renal, lung, cutaneous, colorectal, endometrial, ovarian and cervical carcinoma ${ }^{31}$. In a study done by Haber et al in colorectal cancer, disease specific mortality was greater in patients with high expression of GLUT1 in tumors $(>50 \%$ of cells were GLUT1 positive) $)^{32}$. Furthermore, GLUT1 expression in colorectal cancer was shown to be associated with a high incidence of lymph node metastasis ${ }^{33}$.

The present study was performed to evaluate the expression of hypoxia regulated proteins and their association with clinicopathological response in colorectal adenocarcinomas. We applied a panel of hypoxia-related endogenous immunohistochemical markers on a series of 133 colorectal cancer tissues, and correlated their expression with morphologic tumor parameters, clinicopathological features and overall patient survival.

\section{Materials and methods}

\section{Patients}

Patients were entered in two multi-center prospective clinical trials between 1979 and 1981 in the Netherlands. One trial was designed to compare patient survival after treatment of colonic cancer by conventional surgery or the notouch isolation technique ${ }^{34}$. The other trial was conducted to compare survival in rectal cancer patients with or without preoperative radiotherapy. At the time the trial was conducted, only surgical removal of the tumors was performed, and adjuvant chemotherapy was not yet standard practice. Although the results from this study can therefore not be extrapolated to current practice, it does enable unbiased study of the influence of hypoxic conditions on tumor biology. In the current study, we included only the patients who did not undergo preoperative radiotherapy. For immunohistochemical analysis tumor tissues from 133 patients with primary colorectal cancer were available. The distribution of age, gender, tumor stage, location and type of tumor, frequency 
of events and mean follow-up time of the patients in this study are representative for the patients in the trial (see Table 6.1).

Table 6.1 Clinicopathological variables of study population.

\begin{tabular}{ll}
\hline Age & \\
$<69$ years & $\mathrm{n}=64(48 \%)$ \\
$>69$ years & $\mathrm{n}=69(52 \%)$ \\
Sex & \\
$\quad$ Male & $\mathrm{n}=55(41 \%)$ \\
$\quad$ Female & $\mathrm{n}=78(59 \%)$ \\
Tumor-size & \\
$\quad<46 \mathrm{~mm}$ & $\mathrm{n}=57(43 \%)$ \\
$>46 \mathrm{~mm}$ & $\mathrm{n}=76(57 \%)$ \\
Tumor location & \\
$\quad \begin{array}{l}\text { Proximal } \\
\text { Distal }\end{array}$ & $\mathrm{n}=52(39 \%)$ \\
Tumor & $\mathrm{n}=81(61 \%)$ \\
$\quad$ Colon & \\
Rectum & $\mathrm{n}=91(68 \%)$ \\
Differentiation & $\mathrm{n}=42(32 \%)$ \\
$\quad$ Well & \\
Moderate/poor & $\mathrm{n}=14(11 \%)$ \\
TNM & $\mathrm{n}=119(89 \%)$ \\
1 & \\
2 & $\mathrm{n}=0 \quad(0 \%)$ \\
3 & $\mathrm{n}=83(63 \%)$ \\
4 & $\mathrm{n}=35(26 \%)$ \\
Tumortype & $\mathrm{n}=15(11 \%)$ \\
Exophytic & \\
Sessile & $\mathrm{n}=15(11 \%)$ \\
Ulcerative & $\mathrm{n}=19(14 \%)$ \\
\hline & $\mathrm{n}=99(74 \%)$ \\
\hline
\end{tabular}

Follow up took place every 3 months during the first three years and every 6 months between three and five years after initial diagnosis and surgery. Standard protocols were followed, with routine blood counts and chemistry studies (including CEA levels) at each visit and liver ultrasound, chest $\mathrm{x}$-ray and colonoscopy annually, to evaluate both recurrence of disease and diseaserelated death. After the initial five year follow up period, during the next years only the time and cause of death were registered. Follow-up was complete for all patients. In the present study, failure was defined as death due to recurrent disease, excluding postoperative mortality within 30 days and non-disease related death.

After surgery, tumor tissues and lymph nodes were fixed in buffered formalin, sectioned, and embedded in paraffin. Experienced pathologists documented the histopathological characteristics of the tumors, including tumor stage, differentiation grade, size, (lymph-)angioinvasion, perineural invasion and 
$112 \mid$ Chapter 6

lymphnode involvement. Tumor stage was defined according to the TNM staging system.

\section{Immunohistochemistry}

Formalin fixed, paraffin-embedded tissues consisting of both normal mucosa and tumor were used for immunohistochemical staining. Serial sections $(4 \mu \mathrm{m})$ from each patient were stained for HIF-1 $\alpha$, HIF-2 $\alpha$, CA9 and GLUT1 after deparaffination. Endogenous peroxidase activity was blocked by pre-incubating in $0.6 \%$ hydrogen peroxide for $20 \mathrm{~min}$.

The following staining protocols for the different antibodies were used:

HIF-1a staining: Antigen retrieval was performed by microwave $750 \mathrm{~W}$ for 2 min. followed by $20 \mathrm{~min}$. at $90 \mathrm{~W}$ in $1 \mathrm{mM}$ TE buffer $\mathrm{pH} 9.0$, followed by $30 \mathrm{~min}$. cooling in buffer. Slides were blocked in $25 \%$ normal serum for $10 \mathrm{~min}$. Sections were incubated overnight $\left(4^{\circ} \mathrm{C}\right)$ with primary antibody HIF-1 $\alpha(1: 120)$ (anti-HIF-1a monoclonal: $610958 \mathrm{BD}, \mathrm{USA}$ ).

HIF-2 $\alpha$ staining: Antigen retrieval was performed by microwave treatment $(750$ W for $20 \mathrm{~min}$ in $1 \mathrm{mM}$ TE buffer $\mathrm{pH} 8.0$ ), followed by $30 \mathrm{~min}$. cooling in buffer. Slides were blocked in $25 \%$ normal serum for $10 \mathrm{~min}$. Sections were incubated with primary antibody HIF-2a (1:500) for 100 min. (anti-HIF2 alpha monoclonal: ab8365 AbCam, UK).

CA9 staining: Slides were blocked in $25 \%$ normal serum for 10 min., then incubated for 45 min. with primary CA9 antibody MoAb M75 (1:50) (anti-human CA9, kindly supplied by Dr. S. Pastorekova) at room temperature ${ }^{27}$.

GLUT1 staining: Microwave treatment $(750 \mathrm{~W}$ for $15 \mathrm{~min}$. in Citrate buffer $\mathrm{pH}$ 6.0 ), followed by $30 \mathrm{~min}$. cooling in buffer. Slides were blocked in $25 \%$ normal serum for $10 \mathrm{~min}$. Sections were incubated (1:100) with primary antibody GLUT1 (rabbit polyclonal anti-human GLUT1, A3536, DakoCytomation, Denmark) for $2 \mathrm{hr}$. followed by incubation with Swine Anti-Rabbit Immunoglobuline/Biotinylated (E0431, DakoCytomation, Denmark) (1:250) for $30 \mathrm{~min}$. at room temperature, and StreptABComplex/HRP (K0377, DakoCytomation, Denmark) (1:200) for $30 \mathrm{~min}$. at room temperature.

As a negative control for all antibodies, TBS buffer instead of primary antibody was used. Visualization was performed using Dako Envision, Peroxidase, mouse System (K4001, DAKO, Denmark). For GLUT1, Diaminobenzidine was applied for $10 \mathrm{~min}$. The slides were counterstained with hematoxylin and mounted.

\section{Evaluation of staining}

Immunohistochemical staining was evaluated on the basis of 1) localisation in tumor epithelial or stromal cells and 2) subcellular localisation. For the tumor stroma, only the tumor-associated stromal cells were taken into account, not 
the tumor infiltrating inflammatory cells or the lamina propia of the normal mucosa.

If nuclear staining was present in $>5 \%$ of the tumor epithelial cells or tumorassociated stromal cells, the sample was considered positive for HIF-1 $\alpha$ and HIF-2 $\alpha$. If membranous staining occurred in $>5 \%$ of the tumor epithelial cells or stromal cells, samples were considered positive for CA9 and GLUT1. Staining results were also checked by determining the extent and intensity of staining. Because this did not change the categorization, we used the $5 \%$ cut-off value for further analysis ${ }^{20}$.

\section{Data analysis}

For the data analyses we used three groups of expression patterns for HIF-1 $\alpha$, HIF-2 $\alpha$, CA9 and GLUT1 expression, namely: 1 Stromal expression (= defined as purely stromal or a combination of stromal and epithelial expression), 2 Epithelial expression (=defined as epithelial only or a combination of stromal and epithelial expression), and 3 Negative.

The correlations between HIF-1 $\alpha$, HIF-2 $\alpha$, CA9, GLUT1 and various clinicopathological parameters were determined by the Pearson Chi-Square and Fisher's exact test as appropriate. To evaluate the relationship between HIF-1a, HIF-2a, CA9, GLUT1 and patient survival, Kaplan-Meier survival curves were calculated. Statistical differences between groups were determined by using the Log-rank test. The endpoint for analyses was overall survival starting from the day of surgery. Independent variables predicting survival were evaluated by the multiple stepwise regression analyses using Cox Regression. The Cox-regression model included the variables: sex, age, tumor size, tumor location, TNM stage, differentiation grade, HIF-1 $\alpha$, HIF-2 $\alpha$, CA9 and GLUT1 (epithelial and stromal expression separately). All p-values are two sided and $p$-values $<0.05$ were considered statistically significant. SPSS 10.0 software was used for data analyses.

To check for differences between colon and rectum tumors, all survival analyses were performed separately for these two categories.

\section{Results}

We evaluated the staining characteristics of the potential hypoxia markers HIF1a, HIF-2a, CA9 and GLUT1 in 133 human colorectal adenocarcinomas. On analyzing these four hypoxia associated proteins, it became clear that many tumors displayed positive staining in the tumor epithelial cells as well as positive staining in tumor- associated stromal cells. We therefore separately evaluated the staining patterns of HIF-1 $\alpha$, HIF-2 $\alpha$, CA9 and GLUT1 in tumor epithelial cells and tumor-associated stromal cells. All tumors showed 
immunohistochemical expression of at least one of the analysed hypoxia markers. Overall HIF-1 $\alpha$ was positive in $43 \%$ of cases, whereas $83 \%$ of the tumors were positive for HIF-2a, $89 \%$ of the tumors showed CA9 protein expression and GLUT1 expression was positive in $85 \%$ of the cases.

\section{Expression of hypoxia proteins in tumor epithelial cells}

In order to evaluate the association of hypoxia and the clinical outcome of colorectal cancer, quantification of the expression of hypoxia markers within the tumor epithelial cell compartment was performed. Overall, 29\% of the patients showed HIF-1a protein expression within the tumor epithelial cells (Figure 6.2A). In these cells, HIF-1 $\alpha$ expression was observed exclusively in the nuclei, often in areas surrounding tumor necrosis (Figure 6.1A), a pattern that is typical of hypoxia induced expression. Surprisingly, none of the 133 tumors showed any staining for HIF-2 $\alpha$ in tumor epithelial cells. This lack of expression may be associated with the intestinal cell lineage as expression was also not detected in any adjacent normal intestinal epithelial cells. Epithelial CA9 expression was observed in $78 \%$ of all tumors (Figure $6.2 \mathrm{C}$ ). Expression in the tumor cells was restricted to a membranous type of expression (Figure 6.1E) but was typically not perinecrotic. GLUT1 was also present in tumor epithelial cells in $83 \%$ of the cases (Figure 6.2D), where it showed a clear and strong membranous staining, typically surrounding areas of tumor necrosis (Figure 6.1G).

\section{Expression of hypoxia proteins in the tumor-associated stromal cells}

Stromal HIF-1a expression was observed in $32 \%$ of tumors, of which $18 \%$ showed combined staining of tumor epithelium and stroma and $14 \%$ showed exclusive staining in the stromal cells (Figure 6.2A). Staining was observed surrounding areas of tumor necrosis (Figure 6.1B) and was confined to the nuclei.

In contrast with the lack of HIF-2 $\alpha$ expression in tumor epithelial cells, HIF-2 $\alpha$ staining was frequently seen within the tumor stroma, both in tumor-associated stromal cells and inflammatory cells (Figure 6.1C and 6.1D). Expression was predominantly nuclear, only occasionally accompanied by additional cytoplasmic staining. In further analysis, HIF-2 $\alpha$ staining of inflammatory cells in the tumor stroma was disregarded, because HIF-2 $\alpha$ expression in inflammatory cells did not influence the effect of HIF-2 $\alpha$ expression in tumorassociated stromal cells on patient outcome (Figure $6.3 \mathrm{~A}$ ). Overall, $83 \%$ of the tumors showed positive staining for HIF-2 $\alpha$ in stromal cells, typically surrounding areas of necrosis (Figure 6.2B). In the remaining $17 \%$ of the tumors where no expression of HIF-2a was observed, some staining was found in inflammatory cells in the lamina propria of the normal mucosa. Stromal 
membranous CA9 expression (Figure $6.1 \mathrm{~F}$ ) was observed in $37 \%$ of all tumors. Interestingly, of the tumors that lacked CA9 expression in tumor epithelial cells, half were positive for CA9 in the stroma (Figure 6.2C). GLUT1 expression was only occasionally detected in stromal cells and restricted to a membranous type of expression (Figure $6.1 \mathrm{H}$ ). Stromal cell expression of GLUT1 was present in $14 \%$ of the cases (Figure 6.2D).

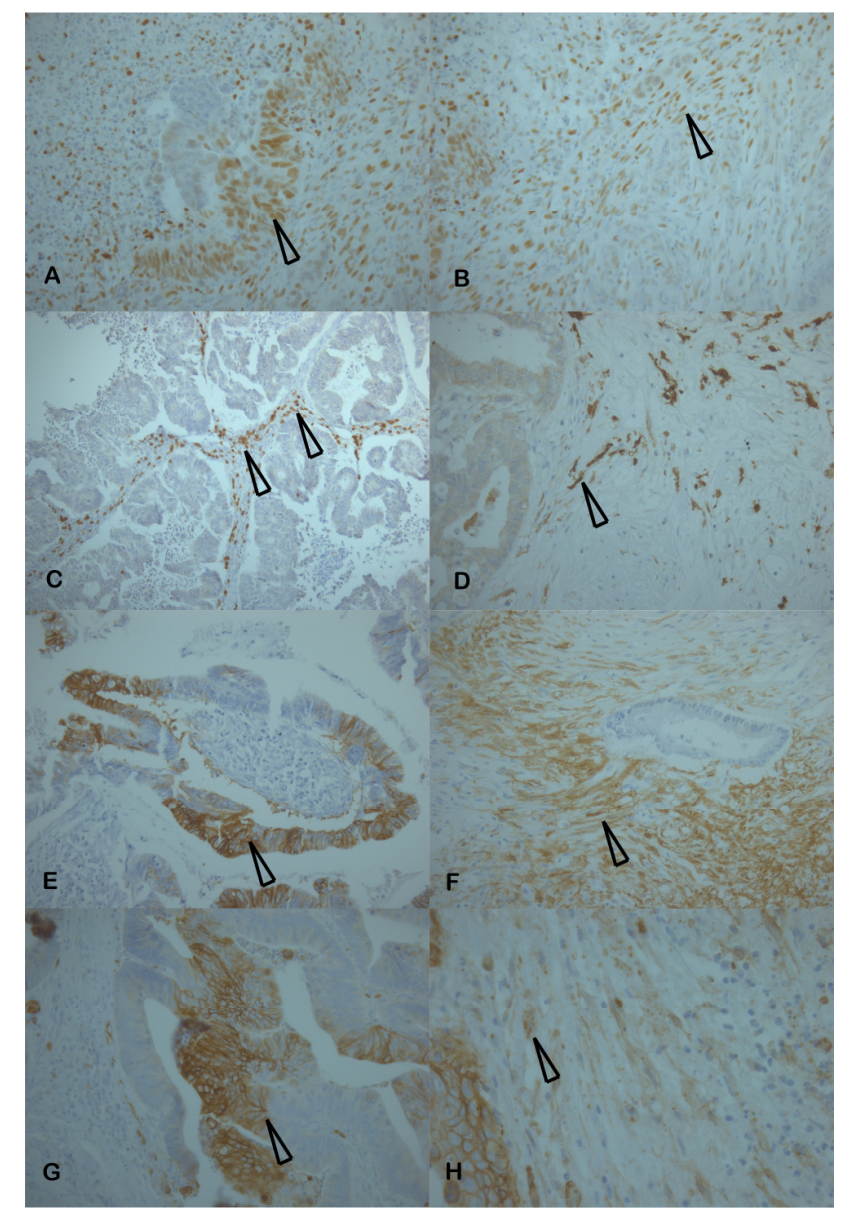

Figure 6.1 Expression patterns of hypoxia regulated proteins in colorectal tumors. black arrow: $(A, E, G)$ epithelial tumor cell expression, $(B, D, F, H)$ tumor-associated stromal cell expresion, (C) inflammatory cell expression. (A) HIF-1a expression in the nuclei of epithelial tumor cells. (B) HIF-1a expression in the nuclei of tumor-associated stromal cells. (C) HIF-2a expression in inflammatory cells located within the tumor-associated stroma. (D) HIF-2 $\alpha$ expression in tumor-associated stromal cells. (E) CA9 expression in epithelial tumor cells. (F) CA9 expression in tumor-associated stromal cells. (G) Expression of GLUT1 in tumor epithelial cells. (H) GLUT1 expression in tumorassociated stromal cells. (A, B, D, F and H original magnifications $\times 40 ; \mathrm{C}, \mathrm{E}$ and $\mathrm{G}$ original magnifications $\times 20$.) 
$116 \mid$ Chapter 6

No staining of stromal cells within the lamina propria of surrounding normal mucosa was found for any of these hypoxia inducible proteins.
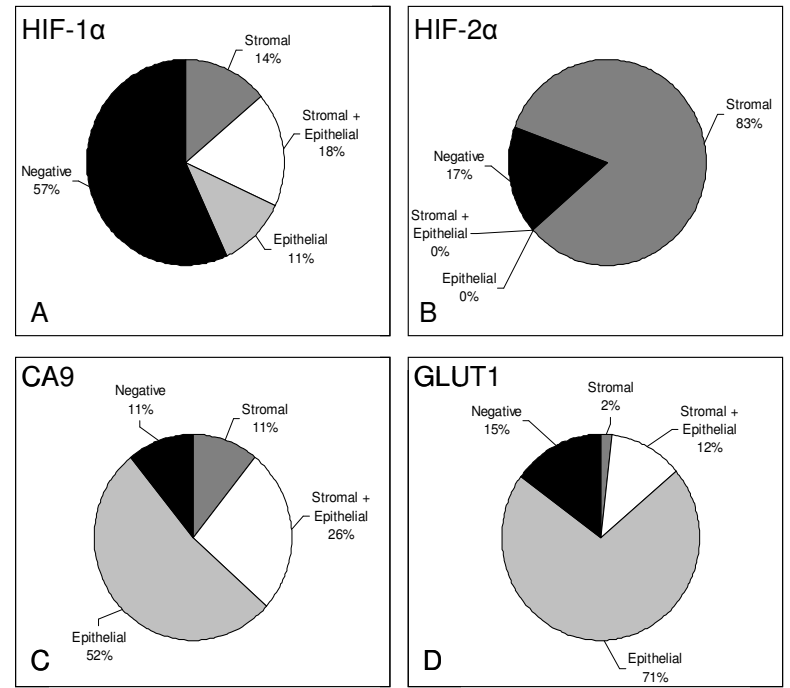

Figure 6.2 Quantification of expression patterns of hypoxia associated proteins in 133 colorectal cancers. (A) Quantification of HIF-1 $\alpha$ expression patterns. (B) Quantification of HIF-2 $\alpha$ expression patterns. (C) Quantification of CA9 expression patterns. (D) Quantification of GLUT1 expression patterns.

\section{Correlations between HIF-1 $\alpha$, HIF-2 $\alpha$, CA9, GLUT1 and clinico- pathological variables}

To investigate the functional relevance of HIF-1 $\alpha$ and HIF-2 $\alpha$ expression, we tested the correlations of the expression of these transcription factors with the expression of their downstream gene products CA9 and GLUT-1. HIF-1a expression showed no correlation with HIF-2 $\alpha$, GLUT1 or CA9 when analyzed by either epithelial or stromal expression patterns. This suggests that HIF-1a expression is not primarily responsible for induction of CA9 and GLUT1 expression and that these genes are regulated by factors other than HIF-1a. In contrast, a significant correlation was found between HIF-2 $\alpha$ and CA9 ( $p=0.02$, Table 6.2) within the tumor stroma. Furthermore, GLUT1 and CA9 expression within the tumor stroma were significantly correlated $(p=0.002$, Table 6.2). These data suggest that HIF-2a upregulation in tumor stroma may be responsible for upregulation of CA9 . 
Stromal expression of hypoxia regulated proteins

Table 6.2 Correlations between stromal expression of HIF-1 $\alpha$, HIF-2a, CA9, GLUT1.

\begin{tabular}{|c|c|c|c|c|c|c|c|}
\hline & \multicolumn{2}{|l|}{ HIF-2a } & \multicolumn{2}{|l|}{$p$-value } & \multicolumn{2}{|l|}{ HIF-1 $1 \alpha$} & \multirow[t]{3}{*}{$\mathrm{p}$-value } \\
\hline & Stroma & Negative & & & Stroma & Negative & \\
\hline HIF-1a & & & & GLUT1 & & & \\
\hline Stroma & $37 / 43(86 \%)$ & 6/43 (14\%) & & Stroma & 5/19 (26\%) & $14 / 19$ (74\%) & \\
\hline \multirow[t]{3}{*}{ Negative } & $74 / 90$ (82\%) & 16/90 (18\%) & 0.6 & Negative & $38 / 114(33 \%)$ & $76 / 114(67 \%)$ & 0.5 \\
\hline & HIF-2a & & & & HIF-1 $\alpha$ & & \\
\hline & Stroma & Negative & & & Stroma & Negative & \\
\hline GLUT1 & & & & CA9 & & & \\
\hline Stroma & 17/19 (89\%) & 2/19 (11\%) & & Stroma & 18/48 (38\%) & $30 / 48$ (62\%) & \\
\hline \multirow[t]{3}{*}{ Negative } & 94/114 (82\%) & $20 / 114$ (18\%) & 0.7 & Negative & $25 / 85$ (29\%) & $60 / 85(71 \%)$ & 0.3 \\
\hline & HIF-2 $\alpha$ & & & & GLUT1 & & \\
\hline & Stroma & Negative & & & Stroma & Negative & \\
\hline CA9 & & & & CA9 & & & \\
\hline Stroma & 45/48 (94\%) & $3 / 48(6 \%)$ & & Stroma & 13/48 (27\%) & 35/48 (73\%) & \\
\hline Negative & $66 / 85(78 \%)$ & $19 / 85(22 \%)$ & 0.02 & Negative & $6 / 85(7 \%)$ & 79/85 (93\%) & 0.002 \\
\hline
\end{tabular}

No significant correlation was found between stromal or epithelial expression of HIF-1 $\alpha$, HIF-2 $\alpha$ or GLUT1 and any of the following clinicopathological characteristics: age, sex, tumor size, location in the colon (proximal, distal), differentiation (well, moderately and poorly), TNM stage (2,3 and 4). Only CA9 epithelial expression showed a significant correlation with TNM stage, with reduced CA9 expression in the more advanced tumors. These results suggest that the biological responses to hypoxia in colorectal cancer are largely independent of these clinicopathological variables.

\section{Relationship between hypoxia markers and patient survival}

An important objective of our study was to analyze the relationship between hypoxia regulated proteins and patient survival. Previous studies in other tumor types have indicated that expression of the hypoxia induced proteins investigated here correlate with poor overall survival. This is presumed to be due to an adverse effect of hypoxia on the tumor cells themselves. Since we also observed frequent staining within the tumor stroma, we further investigated the potential relationship between stromal hypoxia and overall survival.

HIF1- $\alpha$ expression in tumor-associated stromal cells and epithelial tumor cells showed no significant correlation with patient survival. Similarly, CA9 epithelial expression had no impact on patient survival. GLUT1 epithelial negative cases showed a significantly poorer survival compared to GLUT1 positive epithelial cases $(p=0.02$, Figure 6.3B). 
A

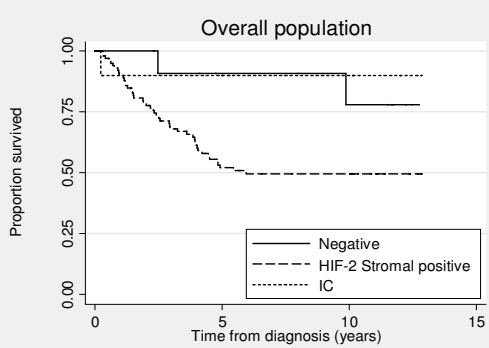

C

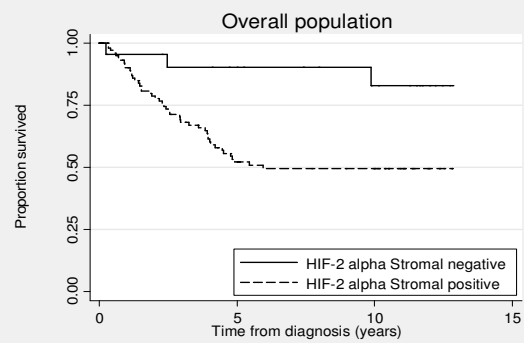

Stage 3

$\mathrm{E}$

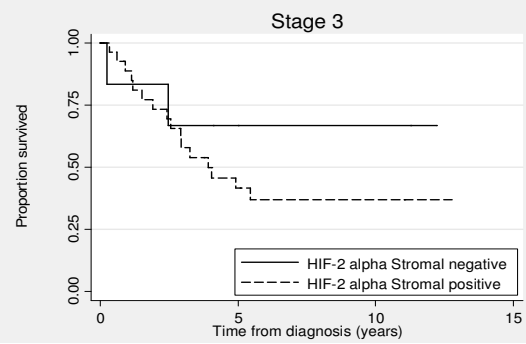

$\mathrm{B}$

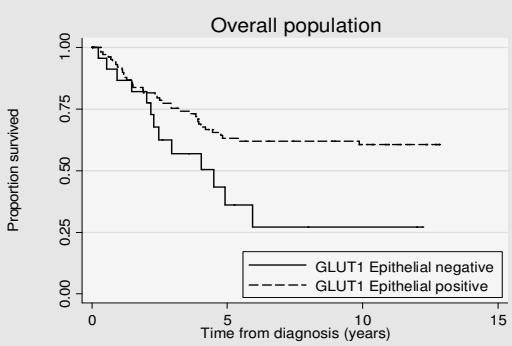

D
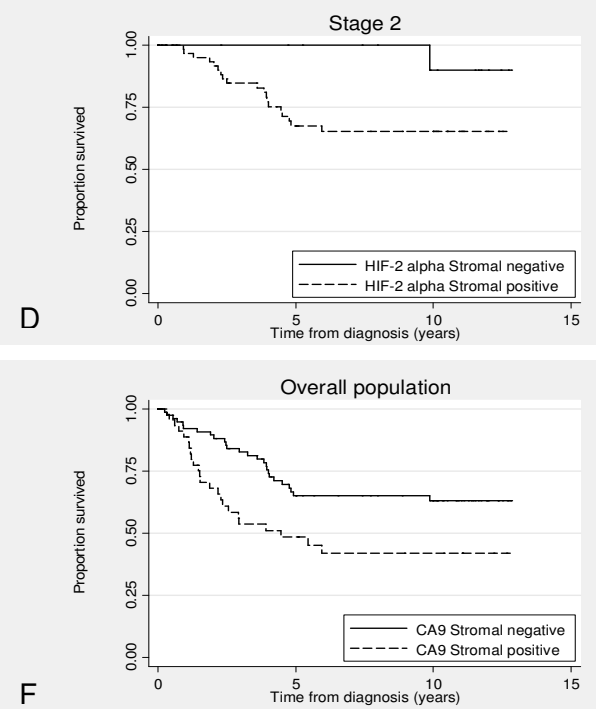

Figure 6.3 (A) Survival curve HIF-2 $\alpha$ expression including inflammatory cells (IC) $p=0.06$ $(n=133)$. (B) Survival curve GLUT1 epithelial expression $p=0.02 \quad(n=133)$. (C) Survival curve HIF-2 $\alpha$ (without inflammatory cells) $p=0.008 \quad(n=133)$. (D) Survival curve HIF-2 $\alpha$ stratified for TNM stage $2 p<0.05(n=83)$. (E) Survival curve HIF-2 $\alpha$ stratified for TNM stage $3 p=0.4(n=35)$. (F) Survival curve CA9 $p=0.01 \quad(n=133)$.

In contrast to the lack of association between markers of hypoxia in the tumor cells and clinical response, we found strong evidence that stromal hypoxia may be associated with poor outcome. Patients with stromal HIF-2 $\alpha$ expression showed a statistically significant poorer survival compared to HIF-2a negative patients $(p=0.008$, Figure $6.3 \mathrm{C}$ ). This trend was maintained in TNM stage 2 and stage 3 (Figure 6.3D and 6.3E). The same trend was observed for stromal CA9 expression ( $p=0.01$, Figure $6.3 F$ ). Patients with stromal GLUT1 expression also showed a tendency for poorer overall survival compared to patients negative for stromal GLUT1 expression, although this did not reach statistical significance. There was no difference in survival between cases with exclusive 
stromal expression and cases with combined stromal and epithelial expression for any of the four applied markers (data not shown).

\section{Multivariate analysis}

An overall model to test the most relevant prognostic factors for patient outcome was performed using a multistep Cox-regression model. After stepwise multivariate analysis, stromal HIF-2a positivity (Hazard Ratio (HR) 4.61), tumor epithelial GLUT1 positivity (HR 0.44), TNM stage 3 (HR 2.74) and stage 4 (HR 16.09) and age >69 years (HR 2.09) were statistically significant independent prognostic markers. Poorly and moderately differentiated tumors had a HR of 3.12 compared to well differentiated tumors, although this did not reach statistical significance $(p=0.07$, Table 6.3). Multivariate analysis restricted to stage 2 and 3 cases, revealed stromal HIF-2a positivity as the strongest independent prognostic marker, with a HR $4.87(p=0.01)$, followed by TNM stage 3 (HR 2.29, $p=0.01$ ), stromal CA9 expression ( $H R$ 1.95, $p<0.05$ ), age $>69$ years $(H R$ 1.94, $p<0.05)$ and epithelial GLUT1 expression (HR 0.26, $\mathrm{p}=0.001)$.

There were no differences in univariate or multivariate survival analysis between colon and rectum tumors.

Table 6.3 Cox proportional hazard model.

\begin{tabular}{llcc}
\hline & & $\begin{array}{c}\text { Event: CRC mortality } \\
\text { HR }(95 \% \mathrm{Cl})\end{array}$ & p-value \\
\hline HIF-2 $\alpha$ & Positive $^{*}$ & $4.61(1.40-15.50)$ & 0.01 \\
GLUT1 & Epithelial $^{\dagger}$ & $0.44(0.22-0.86)$ & 0.02 \\
TNM & $3^{\ddagger}$ & $2.74(1.42-5.29)$ & 0.003 \\
& $4^{\ddagger}$ & $16.09(7.00-39.95)$ & 0.000 \\
Differentiation & Moderately/poorly $^{\star *}$ & $3.12(0.93-10.48)$ & 0.07 \\
Age & $>69$ years $^{\dagger+}$ & $2.09(1.16-3.74)$ & 0.01 \\
\hline
\end{tabular}

$\mathrm{CRC}$, colorectal cancer; HR, Hazard Ratio=Relative Risk. ${ }^{*}$ Reference group = negative HIF-2 $\alpha$ cases; ${ }^{\dagger}$ Reference group = negative GLUT1 cases; ${ }^{\ddagger}$ Reference group $=$ TNM stage $2 ;{ }^{* *}$ Reference group $=$ well differentiated; ${ }^{+\dagger}$ Reference group $=$ Age $<69$ years .

\section{Discussion}

This is the first comprehensive study analyzing the expression of endogenous hypoxia markers HIF-1 $\alpha$, HIF-2a, CA9 and GLUT1 in colorectal cancer. All 133 tumors in this study showed expression of at least one of the hypoxia-regulated proteins, suggesting that all tumors may exhibit at least some degree of hypoxia. In contrast to what was initially expected, our data suggest that HIF-1 $\alpha$ immunostaining does not appear to be a reliable indicator of hypoxia in colorectal cancer and furthermore does not correlate with patient outcome. Although HIF-1 $\alpha$ did display staining characteristics consistent with hypoxia 
(peri-necrotic staining of cell nuclei), its expression in either stromal or tumor cells did not correlate with the expression of its downstream genes CA9 and GLUT1. Consistent with our results, HIF-1a staining was previously reported to be positive in $44.8 \%$ of patients with colorectal cancer, but had no correlation with HIF-2 $\alpha$ expression and no prognostic value ${ }^{5}$. Several explanations for the lack of correlation between HIF-1 $\alpha$ and CA9 or GLUT1 in the tumor cells are possible. HIF-1 $\alpha$ is susceptible to degradation after prolonged hypoxia due to upregulation of the Prolyl Hydroxylase Domain proteins ${ }^{35}$. In vitro studies suggest that nutrient deprivation may also decrease HIF-1a expression. Furthermore, the half-life of CA9 is sufficiently long ( $>>24 \mathrm{hrs}$ ) that once formed it remains present for many days in the absence of continued HIF-1 $\alpha$ expression $^{36}$.

As for HIF-1 $\alpha$, our data indicate that tumor epithelial cell expression of CA9, although frequently occurring, is not related to poorer survival either. In fact, we found that epithelial CA9 expression decreased with advancing TNM stage, with $78 \%$ of CA9 epithelial positive cases in TNM stage 2 compared to $53 \%$ of CA9 epithelial positive cases in TNM stage $4(p=0.02)$. It is well known that in colorectal cancer TNM Stage 2 tumors have a better overall survival than Stage 4 tumors $^{37}$. The lack of correlation between HIF-1 $\alpha$ and CA9 expression in tumor epithelial cells suggests that the CA9 positivity may also be unrelated to hypoxia. This is supported by the finding that in cases with only tumor epithelial CA9 staining (53\%), the expression was usually not perinecrotic. On the other hand, tumor epithelial cell expression of GLUT1 in our study was typically found in perinecrotic zones, and therefore may indeed be indicative of tumor hypoxia. However, GLUT1 epithelial positivity turned out to be a marker for good prognosis compared to epithelial negative GLUT1 cases. This is somewhat surprising, since GLUT1 expression has been previously reported to be associated with a high incidence of lymphnode metastases ${ }^{38}$. Although further analyses is clearly necessary to determine the role for epithelial GLUT1 expression within different TNM stages of colorectal cancer, our data do not support the premise that epithelial GLUT1 expression is a poor prognostic factor. Taken together, the immunostaining patterns of these four markers within the tumor epithelial cell compartment do not consistently reflect tumor hypoxia, and tumor epithelial cell hypoxia does not seem to contribute to a poorer patient prognosis in colorectal cancer.

In direct contrast to these results, our study did indicate that the tumor stroma may be frequently hypoxic. We found that $84 \%$ of all tumors demonstrated staining of HIF-2 $\alpha$ within the tumor-associated stromal cells. HIF-2 $\alpha$ appears to be biologically active in stromal cells because HIF-2 $\alpha$ correlated significantly with stromal CA9 expression $(\mathrm{p}=0.02)$ in these tumors. Most importantly, tumors showing stromal HIF-2 $\alpha$ expression also had a worse overall survival when compared with HIF-2 $\alpha$ negative cases. After stratification for other factors 
in a multivariate analysis (sex, age, TNM stage, differentiation grade, HIF-1a, GLUT1 and CA9), stromal HIF-2 $\alpha$ expression was a strong (HR 4.61, $p=0.01$ ) independent unfavorable prognostic factor. The negative impact of HIF-2 $\alpha$ expression on patient survival in colorectal cancer was recently also described by others. However, in this study of curatively resected colorectal carcinoma patients, the authors did not study the contribution of the epithelial and stromal tumor compartments separately ${ }^{20}$.

As might be expected given the correlation between HIF-2 $\alpha$ and CA9 in tumorassociated stromal cells, our data also indicated that stromal CA9 expression was associated with a worse overall survival. Furthermore, stromal CA9 expression proved to be an independent unfavorable prognostic factor in cases having stage 2 or 3 colorectal cancer. This finding may not be unique to colorectal cancer. In invasive breast cancer, stromal CA9 expression levels have been associated with a higher relapse rate and a worse overall survival ${ }^{39}$. Together our data suggest the intriguing possibility that hypoxia within the tumor associated stroma may contribute to a poor outcome in patients with colorectal cancer who are treated by surgery alone. Although it is commonly accepted that the genetic and epigenetic changes in tumor cells are responsible for the differences in patient response, there are also data to suggest that non-tumor cells may play an important role as well. The induction of CA9 by hypoxia could help in maintaining normal intracellular $\mathrm{pH}$, preventing apoptosis, and thus provide a survival advantage to the stromal cells, and indirectly be of benefit to the adjacent tumor cells. A reduction in extracellular $\mathrm{pH}$ might also provide an advantage to tumor cells as it helps in breakdown of extracellular matrix, migration and invasion of tumor cells, as well as induction and expression of growth factors. This explanation is consistent with a report from Sivridis et al., who showed that cancer-stromal cell interactions may be favoured by the altered microenvironmental conditions of hypoxia and acidity 40 . The strong association of markers of intratumoral hypoxia and $\mathrm{pH}$ within the tumor stroma indicates an interesting link between cancer cell metabolism and the induction of a supportive stroma that favors cancer cell invasion and migration $^{30,31,41-44}$.

Recent data have provided further compelling evidence that tumor-associated stromal cells are important in cancer growth and metastasis ${ }^{45}$. In colorectal tumors not only epithelial but also stromal elements demonstrate genetic instability and that such stromal alterations might influence the genesis of sporadic colorectal carcinomas ${ }^{46}$. Weinberg et al. showed that co-injection of carcinoma associated stromal cells (myofibroblasts) promoted the growth of invasive breast carcinomas. This effect was mediated in part through increased secretion of stromal cell-derived factor-1 (SDF-1). This is particularly interesting since SDF-1 and its receptor have been shown to be upregulated by hypoxia ${ }^{47}$. The Weinberg study also indicated that the tumor associated stromal cells 
could promote angiogenesis by recruiting endothelial progenitor cells (EPC's) into carcinomas ${ }^{48}$. Stromal cells within the tumor microenvironment have been shown to acquire new properties, including the capacity to promote phenotypic and genetic progression in adjacent epithelial cells ${ }^{30,49}$. For example, epigenetic changes have been shown to occur in stromal cells of breast cancer during tumorigenesis, in a tumor stage- and cell type-specific manner ${ }^{50}$. Alterations of stromal cells may therefore be either a consequence or contributor to an abnormal tumor micro-environment. Our study indicates that hypoxia may be a potential mechanism influencing the phenotype of tumor stroma, ultimately leading to a worse patient prognosis. 
Stromal expression of hypoxia regulated proteins

\section{References}

1. Menon C, Fraker DL: Tumor oxygenation status as a prognostic marker. Cancer Letters 221:225-35, 2005

2. Hockel M, Vaupel P: Tumor hypoxia: definitions and current clinical, biologic, and molecular aspects. J Natl Cancer Inst 93:266-76, 2001

3. Pouyssegur J, Dayan F, Mazure NM: Hypoxia signalling in cancer and approaches to enforce tumour regression. Nature 441:437-43, 2006

4. Koukourakis MI, Giatromanolaki A, Polychronidis A, et al: Endogenous markers of hypoxia/anaerobic metabolism and anemia in primary colorectal cancer. Cancer Sci 97:5828,2006

5. Koukourakis Ml, Giatromanolaki A, Sivridis E, et al: Lactate dehydrogenase 5 expression in operable colorectal cancer: strong association with survival and activated vascular endothelial growth factor pathway--a report of the Tumour Angiogenesis Research Group. J Clin Oncol 24:4301-8, 2006

6. Wouters BG, Van den Beucken T, Magagnin MG, et al: Targeting hypoxia tolerance in cancer. Drug Resistance Updates 7:25-49, 2004

7. Vaupel P, Schlenger K, Knoop C, et al: Oxygenation of human tumors: evaluation of tissue oxygen distribution in breast cancers by computerized $\mathrm{O} 2$ tension measurements. Cancer research 51:3316-3322, 1991

8. Aboagye EO, Maxwell RJ, Kelson AB, et al: Preclinical evaluation of the fluorinated 2nitroimidazole $\mathrm{N}$-(2-hydroxy-3,3,3-trifluoropropyl)-2-(2-nitro-1-imidazolyl) acetamide (SR4554) as a probe for the measurement of tumor hypoxia. Cancer research 57:3314-3318, 1997

9. Bussink J, Kaanders JHAM, van der Kogel AJ: Tumor hypoxia at the micro-regional level: clinical relevance and predictive value of exogenous and endogenous hypoxic cell markers. Radiotherapy and Oncology 67:3-15, 2003

10. Kuwai T, Kitadai $Y$, Tanaka $S$, et al: Expression of hypoxia-inducible factor-1 alpha is associated with tumor vascularisation in human colorectal carcinoma. Int. J.Cancer 105:176181,2003

11. Poellinger L, Johnson RS: HIF-1 and hypoxic response: the plot thickens. Current Opinion in Genetics \& Development 14:81-85, 2004

12. Koong AC, Denko NC, Hudson KM, et al: Candidate genes for the Hypoxic Tumor Phenotype. Cancer research 60:883-887, 2000

13. Schmaltz C, Harrigan Hardenbergh P, Wells A, et al: Regulation of Proliferation-Survival Decisions during Tumor Cell Hypoxia. Molecular and Cellular Biology 18:2845-54, 1998

14. Ravi R, Mookerjee B, Bhujwalla ZW, et al: Regulation of tumor angiogenesis by p53-induced degradation of hypoxia-inducible factor 1 alpha. GENES \& DEVELOPMENT 14:34-44, 2000

15. Tan C, Noronha de RG, Roecker AJ, et al: Identification of a Novel Small-Molecule Inhibitor of the Hypoxia-Inducible Factor1 Pathway. Cancer research 65:605-612, 2005

16. Hoskin PJ, Sibtain A, Daley FM, et al: GLUT1 and CA9 as intrinsic markers of hypoxia in bladder cancer: relationship with vascularity and proliferation as predictors of outcome in ARCON. Britisch Journal of Cancer 89:1290-1297, 2003

17. Park SK, Dadak AM, Haase VH, et al: Hypoxia-induced gene expression occurs solely through the action of hypoxia-inducible factor 1 alpha (HIF-1alpha): role of cytoplasmic trapping of HIF-2 alpha. Molecular Celll Biology 23:4959-71, 2003

18. Sivridis E, Giatromanolaki A, Gatter K, et al: Association of Hypoxia-Inducible Factors 1 alpha and 2 alpha with Activated Angiogenic Pathways and Prognosis in Patients with Endometrial Carcinoma. Cancer 95:1055-1063, 2002

19. Shannon AM, Bouchier-Hayes DJ, Condron CM, et al: Tumour hypoxia, chemotherapeutic resistance and hypoxia-related therapies. Cancer Treat Rev 29:297-307, 2003

20. Yoshimura H, Dhar DK, Kohno H, et al: Prognostic Impact of Hypoxia-INducible Factors 1 alpha and 2 alpha in Colorectal Cancer Pateints: Correlation with tumor Angiogenesis and Cyclooxygenase-2 Expression. Clinical Cancer Research 10:8554-8560, 2004 
21. Opavsky R, Pastorekova S, Zelnik V, et al: Human MN/CA9 Gene, a Novel Member of Carbonic Anhydrase Family: Structure and Exon to Protein Domain Relationships. Genomics 33:480-487, 1996

22. Loncaster JA, Harris AL, Davidson SE, et al: Carbonic Anhydrase (CAIX) Expression, a Potential New Intrinsic Marker of Hypoxia: Correlations with Tumor Oxygen Measurements and Prognosis in Locally Advanced Carcinoma of the Cervix. Cancer research 61:6394-6399, 2001

23. Beasley NJP, Wykoff CC, Watson PH, et al: Carbonic Anhydrase IX, an Endogenous Hypoxia Marker, Expression in Head and Neck Squamous Cell Carcinoma and its Relationship to Hypoxia, Necrosis, and Microvessel Density. Cancer research 61:5262-5267, 2001

24. Wykoff CC, Beasley NJ, Watson PH, et al: Hypoxia-inducible Expression of Tumorassociated Carbonic Anhydrases. Cancer research 60:7075-7083, 2000

25. Pastorek J, Pastorekova S, Callebaut I, et al: Cloning and characterization of MN, a human tumor-associated protein with a domain homologous to carbonic anhydrase and a putative helix-loop-helix DNA binding segment. Oncogene 9:2877-88, 1994

26. Swinson DEB, Jones JL, Richardson D, et al: Carbonic Anhydrase IX Expression, a Novel Surrogate Marker of Tumor Hypoxia, Is Associated With Poor Prognosis in Non-Small-Cell Lung Cancer. Journal of Clinical Oncology 21:473-482, 2003

27. Turner KJ, Crew JP, Wykoff CC, et al: The hypoxia-inducible genes VEGF and CA9 are differentially regulated in superficial vs invasive bladder cancer. Britisch Journal of Cancer 86:1276-82, 2002

28. Robertson N, Potter C, Harris AL: Role of Carbonic Anhydrase IX in Human Tumor Cell Growth, Survival, and Invasion. Cancer research 64:6160-6165, 2004

29. Saarnio J, Parkkila S, Parkkila A, et al: Immuohistochemical Study of Colorectal Tumors for Expression of a Novel Transmembrane Carbonic Anhydrase, MN/CA IX, with Potential Value as a Marker of Cell Proliferation. American Journal of Pathology 153:279-85, 1998

30. Mueller MM, Fusenig NE: Friends or foes - bipolar effects of the tumour stroma in cancer. Nat Rev Cancer 4:839-49, 2004

31. Koukourakis MI, Giatromanolaki A, Harris AL, et al: Comparison of metabolic pathways between cancer cells and stromal cells in colorectal carcinomas: a metabolic survival role for tumor-associated stroma. Cancer Res 66:632-7, 2006

32. Haber RS, Rathan A, Weiser KR, et al: GLUT1 glucose transporter expression in colorectal carcinoma: a marker for poor prognosis. Cancer 83:34-40, 1998

33. Sakashita M, Aoyama N, Minami R, et al: Glut1 expression in T1 and T2 stage colorectal carcinomas: its relationship to clinicopathological features. European Journal of Cancer 37:204-209, 2001

34. Wiggers $\mathrm{T}$, Jeekel $\mathrm{J}$, Arends $\mathrm{JW}$, et al: No-touch isolation technique in colon cancer: a controlled prospective trial. Br J Surg 75:409-15, 1988

35. Uchida T, Rossignol F, Matthay MA, et al: Prolonged Hypoxia Differentially Regulates Hypoxia-inducible Factor (HIF)-1alpha and HIF-2 alpha Expression in Lung Epithelial Cells. The Journal of Biological Chemistry 279:14871-14878, 2004

36. Sobhanifar S, Aquino-Parsons C, Stanbridge EJ, et al: Reduced Expression of HypoxiaInducible Factor-1 alpha in Perinecrotic Regions of Solid Tumors. Cancer Research 65:72597266, 2005

37. Roncucci L, Fante R, Losi L, et al: Survival for Colon and Rectal Cancer in a Populationbased Cancer Registry. European Journal of Cancer 32:295-302, 1996

38. Younes M, Lechago LV, Lechago J: Overexpression of the human erytrocyte glucose transporter occurs as a late event in human colorectal carginogenesis and is associated with an increased incidence of lymph node metastases. Clinical Cancer Research 2:1151-4, 1996

39. Colpaert CG, Vermeuelen PB, Fox SB, et al: The presence of a fibrotic focus in invasive breast carcinoma correlates with the expression of carbonic anhydrase IX and is a marker of hypoxia and poor prognosis. Breast Cancer Research and Treatment 81:137-147, 2003

40. Sivridis E, Giatromanolaki A, Koukourakis MI: Proliferating fibroblasts at the invading tumour edje of colorectal adenocarcinomas are associated with endogenous markers of hypoxia, acidity, and oxidative stress. Journal of Clinical Pathology 58:1033-1038, 2005 
41. Koukourakis MI, Giatromanolaki A, Brekken RA, et al: Enhanced expression of SPARC/osteonectin in the tumor-associated stroma of non-small cell lung cancer is correlated with markers of hypoxia/acidity and with poor prognosis of patients. Cancer Res 63:5376-80, 2003

42. Ingram N, Porter CD: Transcriptional targeting of acute hypoxia in the tumour stroma is a novel and viable strategy for cancer gene therapy. Gene Therapy 12:1058-1069, 2005

43. Vosseler S, Mirancea N, Bohlen P, et al: Angiogenesis Inhibition by Vascular Enothelial Growth Factor Receptor-2 Blockade Reduces Stromal Matrix Metalloproteinase Expression, Normalizes Stromal Tissue, and Reverts Epithelial Tumor Phenotype in Surface Heterotransplants. Cancer Research 65:1294-1305, 2005

44. Lussier C, Sodek J, Beaulieu J: Expression of SPARC/Osteonectin/BM40 in the Human Gut: Predominance in the Stroma of the Remodeling Distal Intestine. Journal of Cellular Biochemistry 81:463-476, 2001

45. Peduto L, Reuter VE, Sehara-Fujisawa A, et al: ADAM12 is highly expressed in carcinomaassociated stroma and is required for mouse prostate tumor progression. Oncogene 25:54626, 2006

46. Ishiguro K, Yoshida $\mathrm{T}$, Yagishita $\mathrm{H}$, et al: Epithelial and stromal genetic instability contributes to genesis of colorectal adenomas. Gut 55:695-702, 2006

47. Ceradini DJ, Kulkarni AR, Callaghan MJ, et al: Progenitor cell trafficking is regulated by hypoxia gradients through HIF-1 induction of SDF-1. Nature medicine 10:858-64, 2004

48. Orimo A, Gupta PB, Sgroi DC, et al: Stromal fibroblasts present in invasive human breast carcinomas promote tumor growth and angiogenesis through elevated SDF-1/CXCL12 secretion. Cell 121:335-48, 2005

49. Joesting MS, Perrin S, Elenbaas B, et al: Identification of SFRP1 as a Candidate Mediator of Stromal-to-Epithelial Signaling in Prostate Cancer. Cancer research 65:10423-10430, 2005

50. Hu M, Yao J, Cai L, et al: Distinct epigenetic changes in the stromal cells of breast cancers. Nature Genetics 37:899-905, 2005 


\section{Chapter 7}

Promoter $\mathrm{CpG}$ island hypermethylation of cancer associated genes is associated with stromal expression of hypoxia markers in colorectal cancer

AHG Cleven

S Derks

$M$ van Engeland

AP de Bruïne 


\section{Abstract}

It is becoming increasingly clear that epigenetic alterations, such as promoter $\mathrm{CpG}$ island methylation of important cancer related genes, play a crucial role in the cellular responses to hypoxia, another hallmark of cancer. This study investigates whether hypoxia in CRC is associated with promoter $\mathrm{CpG}$ island methylation of genes involved in important tumor biological processes such as genomic instability, limitless replication potential, tissue invasion and metastasis, insensitivity to anti-growth signals, self-sufficiency in growth signals, sustained angiogenesis and evading apoptosis.

Expression of HIF-1 $\alpha$, HIF-2 $\alpha$, CA9 and GLUT1 was quantified by immunohistochemistry in 173 colorectal adenocarcinomas. Promoter $\mathrm{CpG}$ island methylation was determined using methylationspecific PCR (MSP) for the following genes: MGMT, CHFR, HLTF, MLH1, RASSF1A (genomic instability), p14ARF, p16INK4A (limitless replication potential), ADAM23, TFPI2 (tissue invasion and metastasis), $A P C$ (insensitivity to anti-growth signals), SOCS1 (self-sufficiency in growth signals), THBS1 (sustained angiogenesis), BNIP3, NEURL, RUNX3, RAB32 (evading apoptosis). Associations between these variables were determined by T-test of means, Pearson Chi-Square and Fisher's exact test as appropriate.

Overall we did not find a correlation between mean number of promoter methylated genes and expression of hypoxia regulated proteins. However, methylation of individual genes was associated with stromal rather than epithelial expression of hypoxia regulated proteins: stromal expression of CA9 was more often observed in CHFR and ADAM23 methylated tumors (52\% (35/67) and 58\% $(35 / 60))$ compared to tumors unmethylated for these two genes $(34 \%(29 / 86)$ and $28 \%(22 / 79)$, respectively $\mathrm{p}=0.03$ and $\mathrm{p}<0.001$ ). Stromal expression of HIF-2 $\alpha$ was more often observed in $A P C$ and SOCS1 methylated tumors (82\% (46/56) and 79\% (77/98), respectively) compared to tumors unmethylated for these two genes (67\% (73/109) and 61\% (39/64) respectively, $p=0.04$ and $\mathrm{p}=0.02)$. Only BNIP3 methylated CRCs showed more often CA9 epithelial expression $(87 \%$ (78/90)) compared to its unmethylated counterpart (73\% (50/69), $\mathrm{p}=0.03)$.

Stromal expression of hypoxia regulated proteins HIF-2 $\alpha$ and CA9 was associated with promoter $\mathrm{CpG}$ island methylation of genes involved in important tumor biological processes. Tumors with hypoxic features do not appear to show increased overall $\mathrm{CpG}$ island promoter methylation, but rather harbour specific methylated cancer biology related genes, which could contribute to the more aggressive behaviour of these tumors. 


\section{Introduction}

Hypoxia has been recognized as one of the fundamental features of solid tumors like colorectal cancer (CRC). It plays a critical role in various cellular and physiologic events, including apoptosis, cell proliferation and angiogenesis ${ }^{1-4}$. Also it is important from a clinical point of view, as hypoxic tumors show resistance to radiation therapy and chemotherapy, and a poorer prognosis by increasing risk of invasion and metastasis ${ }^{1-4}$. The cellular responses to hypoxia are at least partially orchestrated by activation of the hypoxia-inducible factors (HIFs) and its many downstream genes including carbonic anhydrase 9 (CA9) and glucose transporter 1 (GLUT1) ${ }^{2}$. In the previous chapter we studied the role of protein expression of HIF-1 $\alpha$, HIF-2 $\alpha$, CA9 and GLUT1 in colorectal adenocarcinomas. We concluded that expression of hypoxia regulated proteins in tumor associated stroma, as opposed to their expression in cancer cells, is associated with poorer outcome, suggesting that tumor hypoxia may influence tumor associated stromal cells in a way that ultimately effects patient prognosis.

It is becoming increasingly apparent that epigenetic alterations, such as promoter $\mathrm{CpG}$ island methylation of important cancer related genes, also play a crucial role in the cellular responses to hypoxia ${ }^{5}$. Hypoxia could be a consequence of tumor progression through increased $\mathrm{CpG}$ island methylation and silencing of important tumor suppressor genes, or conversely, hypoxia could induce cancer related epigenetic changes. Such epigenetic changes may co-operate with hypoxia induced proteins to contribute to the maintenance of a hypoxia-adapted cellular phenotype, which is associated with an aggressive tumor behavior ${ }^{5}$. There are four current opinions on the interaction of epigenetics and hypoxia: (1) HIF stabilisation is influenced by the epigenetically controlled expression of several HIF-degrading proteins, for example silencing of $\mathrm{VHL}$ by promoter $\mathrm{CpG}$ island hypermethylation in renal cell carcinomas ${ }^{6}$. (2) Epigenetic mechanisms such as histone acetyltransferase enzymes regulate HIF binding by maintaining a transcriptionally active chromatin confirmation within and around HIF binding regions ${ }^{7}$. (3) Histone demethylase enzymes are direct HIF target genes and therefore play a role in the regulation of transcription during the hypoxic response ${ }^{8}$. (4) Significant global changes in histone modifications and promoter $\mathrm{CpG}$ island methylation occur in response to hypoxic exposure.

Studying the relation between hypoxia and epigenetic mechanisms should address the notion that the extent of promoter $\mathrm{CpG}$ island methylation of tumor suppressor genes and other important cancer related genes influences the ability of a tumor to adapt to hypoxia ${ }^{5,9}$. An example of this phenomenon is found in both $\mathrm{CRC}$ and pancreatic cancer, where hypoxic induction of $\mathrm{BCL}$ 2/adenovirus E1B-19 kDA-interacting protein (BNIP3), which is known to 
promote apoptosis, is blocked by promoter $\mathrm{CpG}$ island methylation, thereby facilitating the evasion of apoptosis during hypoxia ${ }^{10,11}$. Previously we observed that promoter $\mathrm{CpG}$ island methylation of $C H F R$ is related to a poorer survival. Loss of function of CHFR causes genomic instability ${ }^{12-14}$, which is a feature of tumor progression leading to increasing hypoxia, but may also contribute to intrinsic resistance of tumor cells to hypoxia ${ }^{4}$. Comparable to promoter $\mathrm{CpG}$ island methylation of $B N I P 3$, silencing of $C H F R$ could contribute to a more efficient adaptation of a tumor to hypoxia, eventually resulting in a more aggressive tumor behavior.

The aim of the current study was to find out whether expression of hypoxia regulated proteins in $\mathrm{CRC}$ is related to promoter $\mathrm{CpG}$ island methylation of 16 genes which are frequently methylated in CRC, and also involved in main pathways of tumor progression, the so-called hallmarks of cancer ${ }^{15}$ (Table 7.1): MGMT, CHFR, HLTF, MLH1, RASSF1A (genomic instability), p14ARF, p16INK4A (limitless replication potential), ADAM23, TFPI2 (tssue invasion and metastasis), APC (insensitivity to anti-growth signals), SOCS1 (self-sufficiency in growth signals), THBS1 (sustained angiogenesis), BNIP3, NEURL, RUNX3, $R A B 32$ (evading apoptosis). Methylation status of these genes was determined using methylation-specific PCR (MSP) in a series of $173 \mathrm{CRCs}$. Expression of HIF-1 $\alpha$, HIF-2 $\alpha$, CA9 and GLUT1 was quantified by immunohistochemistry in this series of CRC's. Since we previously described the importance of stromal expression of hypoxia regulated proteins for patient survival in CRC, in the current study we also investigated whether promoter $\mathrm{CpG}$ island hypermethylation was related to differential expression of hypoxia regulated proteins, in either the epithelial or stromal tumor compartments.

Table 7.1 Promoter CpG island hypermethylated genes related to the hallmarks of cancer

\begin{tabular}{lll}
\hline Cancer Hallmarks & Promoter CpG island hypermethylated genes & Reference \\
\hline Genomic instability & MGMT, CHFR, HLTF, MLH1, RASSF1A & $12-14,40-56$ \\
Limitless replication potential & p14ARF, p16INK4A & $57-60$ \\
Tissue invasion and metastasis & ADAM23, TFPI2 & $15,61-68$ \\
Insensitivity to anti-growth signals & APC & $69-71$ \\
Self-sufficiency in growth signals & SOCS1 & $72-74$ \\
Sustained angiogenesis & THBS1 & $75-77$ \\
Evading apoptosis & BNIP3, NEURL, RUNX3, RAB32 & $78-86$ 10,37-39,87-91 \\
\hline
\end{tabular}

\section{Materials and methods}

\section{Patient population}

Patients were entered in two multi-center prospective clinical trials in the Netherlands, between 1979 and 1981. One trial was designed to compare patient survival after treatment of colon cancer by conventional surgery or the 
no-touch isolation technique ${ }^{16}$. The second trial was conducted to compare survival in rectal cancer patients with or without preoperative radiotherapy. In the current study, we included only the patients who did not undergo preoperative radiotherapy. At the time the trial was conducted, only surgical removal of the tumors was performed, and adjuvant chemotherapy was not yet standard practice. This study population therefore enables unbiased study of the influence of hypoxic conditions on tumor biology.

Tumor tissues were fixed in buffered formalin, sectioned, and embedded in paraffin. Experienced pathologists documented the histopathological characteristics of the tumors (see previous chapters). Follow up took place every 3 months during the first three years and every 6 months between three and five years after initial diagnosis and surgery. Standard protocols were followed, with routine blood counts and chemistry studies (including CEA levels) at each visit and liver ultrasound, chest x-ray and colonoscopy annually, to evaluate recurrence of disease and disease-related death. After five year follow up period, only time and cause of death were registered. Follow-up was complete for all patients. Failure was defined as death due to recurrent disease, excluding postoperative mortality within 30 days and non-disease related death.

For immunohistochemical and molecular analysis, tumor tissues from $173 \mathrm{CRC}$ patients were available. The distribution of age, gender, tumor stage, location and type of tumor, frequency of events and mean follow-up time of the patients in this study are representative for the patients in the trial.

\section{Immunohistochemistry}

Immunohistochemistry and evaluation of the data was performed as described previously ${ }^{17}$. In brief serial formalin fixed, paraffin-embedded tissues sections $(4 \mu \mathrm{m})$ were stained for HIF-1 $\alpha$, HIF-2 $\alpha$, CA9 and GLUT1. Localisation (epithelial or stromal) was scored separately. For the category stromal staining only the stromal myofibroblasts were taken into account, not the tumor infiltrating inflammatory cells or the lamina propia of the normal mucosa. If staining was present in $>5 \%$ of the tumor stromal cells, the sample was considered positive for stromal expression. If membranous staining occurred in $>5 \%$ of the tumor epithelial cells, samples were considered positive for epithelial expression ${ }^{18}$.

\section{Promoter CpG island methylation analysis}

Genomic DNA was extracted from CRC tissues using PureGene ${ }^{\mathrm{TM}}$ Genomic DNA Isolation Kit (Gentra Systems) according to the manufacturer's protocol. Promoter $\mathrm{CpG}$ island methylation of $\mathrm{MLH1}$ (mutL homolog1, colon cancer, nonpolyposis type 2 (E.Coli)), p16INK4A (cyclin dependent kinase inhibitor 2A), 
$132 \mid$ Chapter 7

p14ARF (cyclin dependent kinase inhibitor 2A), MGMT (O-6-methylguanineDNA methyltransferase), RASSF1A (Ras association (RalGDS/AF-6) domain family member 1), APC (adenomatous polyposis coli), HLTF (helicase-like transcription factor), CHFR (checkpoint with forkhead and ring finger domain), ADAM23 (ADAM metallopeptidase domain 23), RAB32 (Rab32, a member RAS oncogene family), BNIP3 (BCL2-adenovirus E1B 19kDA interacting protein 3), NEURL (neuralized homolog (Drosophila)), THBS1 (thrombospondin 1), TFPI2 (tissue factor inhibitor 2), RUNX3 (runt-related transcription factor 3 ) and SOCS1 (suppressor of cytokine signaling 1) was determined using sodium bisulfite modification of genomic DNA using the EZ DNA methylation kit (ZYMO research Co., Orange, CA). Methylation Specific PCR (MSP) was performed as described in detail elsewhere ${ }^{19,20}$. In brief, to facilitate MSP analysis on DNA retrieved from formalin-fixed, paraffin-embedded tissue, DNA was first amplified with flanking PCR primers that amplify bisulfite modified DNA but do not preferentially amplify methylated or unmethylated DNA. The resulting fragment was used as a template for the MSP-reaction. All PCRs were performed with a control for unmethylated alleles (normal lymphocyte DNA) and a positive control for methylated alleles (Sssl methyltransferase (New England Biolabs) treated normal lymphocyte DNA) and a negative control without DNA. Each PCR reaction was loaded onto a $2 \%$ agarose gel, stained with Gelstar $^{\circledR}$ (Cambrex Bioscience Rockland Inc, USA) and visualized under UV illumination. For primers and PCR conditions see chapter 4.

\section{Data-analysis}

The Methylation Index ( $\mathrm{Ml}=$ number of methylated promoter $\mathrm{CpG}$ islands divided by number of promoter $\mathrm{CpG}$ islands successfully analyzed) was calculated using the promoter $\mathrm{CpG}$ islands of 16 genes (Table 7.1). Relationships between mean methylation index and expression of HIF-1 $\alpha$, HIF$2 \alpha$, CA9, GLUT1 was determined by T-test of means. Correlations between HIF-1 $\alpha$, HIF-2 $\alpha$, CA9, GLUT1 and the cancer hallmark genes were determined by the Pearson Chi-Square and Fisher's exact test as appropriate. All p-values are two sided and $p$-values $<0.05$ were considered statistically significant. Patients with unknown and unspecified scores have been omitted from analyses for that specific variable. SPSS 15.0 software was used for data analyses.

\section{Results}

Overall, no correlation was observed between epithelial and stromal expression of HIF-1 $\alpha$, HIF-2 $\alpha$, CA9 and GLUT1 and the methylation index in these subgroups of $\mathrm{CRC}$, which varies between 0.36 and 0.45 (Table 7.2). However 
when categorizing each gene in important tumor biology pathways (Table 7.1), we found that promoter $\mathrm{CpG}$ island methylation of a least one gene within each group was associated with stromal expression of hypoxia regulated proteins but not with epithelial expression (Table 7.3). Within the group of genomic instability associated genes (MGMT, CHFR, HLTF, MLH1, RASSF1A), stromal expression of hypoxia regulated protein CA9 was more often observed in CHFR methylated tumors $52 \%$ (35/67) compared to unmethylated CHFR tumors $34 \%$ (29/86), $p=0.03$. Epithelial expression of hypoxia regulated proteins was not related to methylation of genes involved in genomic instability. Within the group of methylated genes involved in limitless replication potential ( $p 14 A R F, p 16 I N K 4 A$ ), we found a borderline significant correlation that stromal expression of HIF-2 $\alpha$ was more often observed in p16INK4A methylated tumors $80 \%$ (56/70) compared to unmethylated p16INK4A tumors $65 \%(58 / 89)$, $\mathrm{p}=0.05$. In line with the group of genes involved in genomic instability, epithelial expression of hypoxia regulated proteins was not associated with the methylation status of genes related to limitless replication potential. Between hypoxia regulated proteins and genes involved in tissue invasion and metastasis (ADAM23, TFPI2), again stromal expression of hypoxia regulated protein CA9 was more often observed in ADAM23 methylated tumors $58 \%$ (35/60) compared to ADAM23 unmethylated tumors $28 \%$ (22/79), $\mathrm{p}<0.001$.

Table 7.2 Mean methylation index (MI) of MGMT, CHFR, HLTF, MLH1, RASSF1A, p14ARF, p16INK4A, ADAM23, TFPI2, APC, SOCS1, THBS1, BNIP3, NEURL, RUNX3, RAB32 according to positive expression of hypoxia regulated proteins in the overall study population.

\begin{tabular}{|c|c|c|c|c|c|}
\hline & MI & $p$-value & & MI & p-value \\
\hline HIF-1a & & & HIF-1a & & \\
\hline Stroma & 0.38 & & Epithelial & 0.43 & \\
\hline Negative & 0.45 & 0.09 & Negative & 0.43 & 0.98 \\
\hline \multicolumn{6}{|l|}{ HIF-2a } \\
\hline Stroma & 0.38 & & & & \\
\hline Negative & 0.35 & 0.39 & & & \\
\hline CA9 & & & CA9 & & \\
\hline Stroma & 0.41 & & Epithelial & 0.39 & \\
\hline Negative & 0.36 & 0.19 & Negative & 0.35 & 0.45 \\
\hline GLUT1 & & & GLUT1 & & \\
\hline Stroma & 0.37 & & Epithelial & 0.40 & \\
\hline Negative & 0.40 & 0.55 & Negative & 0.40 & 0.95 \\
\hline
\end{tabular}

Likewise $A P C$, which is involved in insensitivity to anti-growth signals, showed more often $82 \%(46 / 56)$ HIF-2 $\alpha$ stromal expression in methylated tumors compared to $A P C$ unmethylated tumors $67 \%$ (73/109), $p=0.04$. Furthermore within the group of genes involved in self-sufficiency in growth signals, HIF-2 $\alpha$ stromal expression was more often observed within SOCS1 methylated tumors 
$79 \%(77 / 98)$ compared to their unmethylated counterparts 61\% (39/64), $\mathrm{p}=0.04$. These results underscore that in particular stromal and not epithelial expression of hypoxia regulated proteins is associated with the loss of function by promoter $\mathrm{CpG}$ island methylation of genes involved in important tumor biological processes like genomic instability, tissue invasion and metastasis. THBS1 promoter $\mathrm{CpG}$ island methylation was an infrequent event within our study population $(n=8)$, and furthermore we did not observe a correlation between hypoxia regulated proteins and THBS1 methylation involved in sustained angiogenesis. Four genes (BNIP3, NEURL, RUNX3, RAB32) were related to evading apoptosis, of which only BNIP3 methylated tumors showed more often $87 \%$ (78/90) CA9 epithelial expression compared to BNIP3 unmethylated tumors $73 \%(50 / 69, p=0.03)$. Within this group of genes, stromal expression of hypoxia regulated proteins was not related to promoter CpG island methylation.

Regarding HIF-1a, neither stromal nor epithelial expression showed correlation with promoter $\mathrm{CpG}$ island methylation of genes listed in Table 7.3. The results for GLUT1 were less clear-cut. Although GLUT1 stromal expression appeared to be a feature of THBS1 methylated tumors $43 \%(3 / 7)$ compared to THBS1 unmethylated tumors $12 \%(13 / 105), p=0.06$, it was more often observed in p16INK4A unmethylated tumors $23 \%$ (15/66) compared to p16INK4A methylated tumors $8 \%(5 / 61), p=0.03$. As already stated in chapter 5 , these findings for GLUT1 should be interpreted with caution, since the frequency of stromal GLUT1 expression is very low $(n=20)$ compared to stromal expression of HIF-2 $\alpha(n=119)$ and CA9 $(n=66)$. 


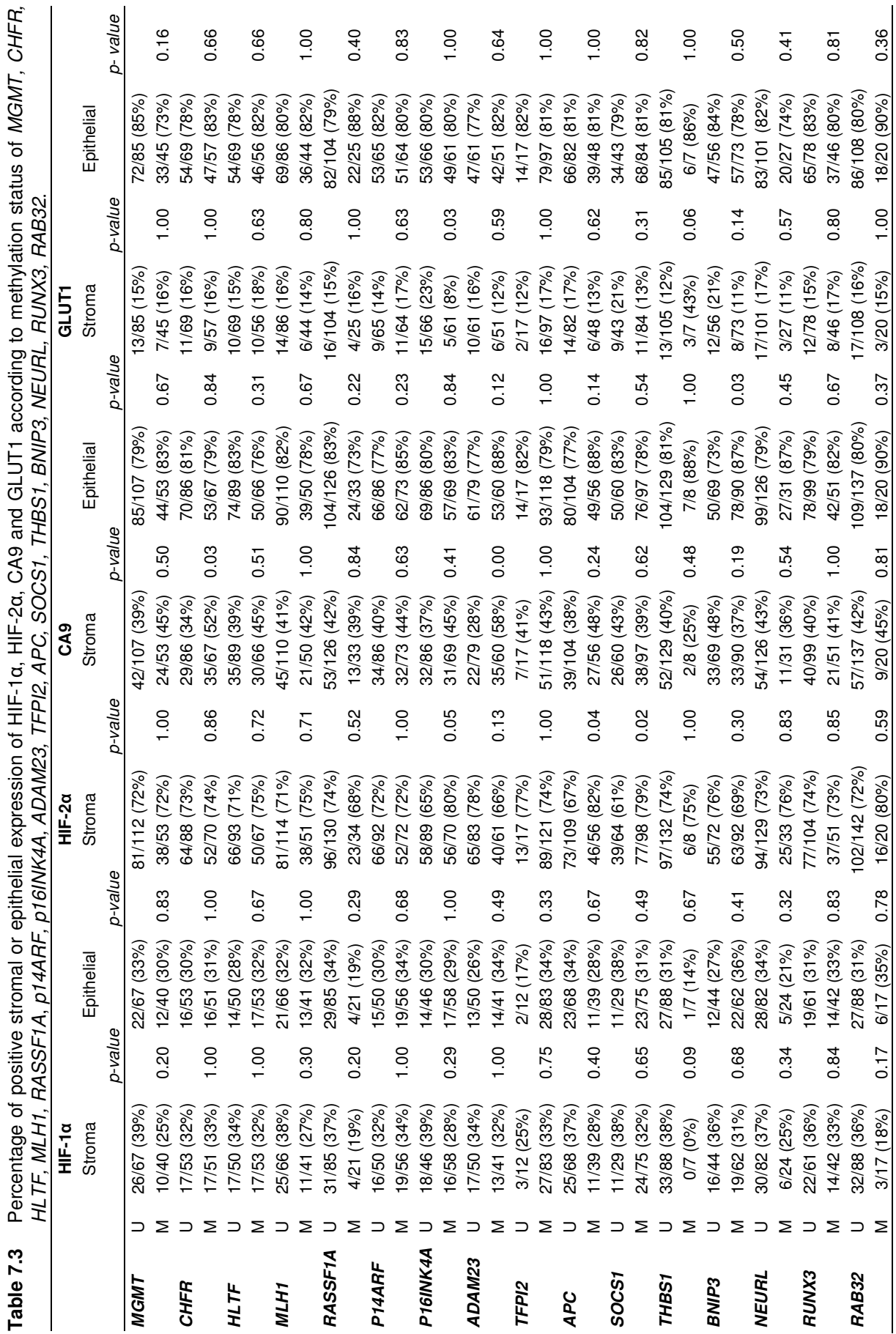




\section{Discussion}

The interactions between epigenetic changes in tumors and the phenomenon of tumor hypoxia are complex, including HIF-dependent and independent mechanisms, and vary from HIF stabilization through epigenetic control of HIF degrading proteins, through activity of histone acetyltransferases and histone demethylases involved in binding and transcriptional activity of HIF, to global epigenetic alterations such as histone modification and promoter $\mathrm{CpG}$ island methylation.

In this study, we emphasized on the latter aspect and formulated the hypothesis that hypoxia in CRC is related to promoter CpG island methylation of genes which play a role in executing important mechanisms of cancer progression, the so-called hallmarks of cancer. We studied a panel of hypoxiarelated markers including HIF-1 $\alpha$, HIF-2 $\alpha$, CA9 and GLUT1, and correlated their expression with promoter $\mathrm{CpG}$ island methylation of 16 genes frequently methylated in CRC including: MGMT, CHFR, HLTF, MLH1, RASSF1A p14ARF, p16INK4A, ADAM23, TFPI2, APC, SOCS1, THBS1, BNIP3, NEURL, RUNX3, RAB32. The results show interesting correlations between hypoxia and DNA promoter hypermethylation. Although overall hypoxia was not associated with a higher methylation index, stromal expression of hypoxia regulated proteins HIF-2 $\alpha$ or CA9 was related to methylation of at least one gene within almost every group of cancer related biological pathways (Table 7.1) as follows: CHFR, $\mathrm{p}=0.03$ (genomic instability), p16INK4A, $\mathrm{p}=0.05$ (limitless replication potential), $A D A M 23, p<0.001$ (tissue invasion and metastasis), $A P C \mathrm{p}=0.04$ (insensitivity to anti-growth signals), SOCS1, $\mathrm{p}=0.04$ (self-sufficiency in growth signals). These results have to be interpreted with caution, since we studied relatively many genes $(n=16)$ within small subgroups of CRCs, possibly resulting in false positive results due to multiple testing. Therefore our results should be interpreted accordantly and need further validation in additional studies ${ }^{21}$. Although our data need to be validated, the fact that expression of HIF-2 $\alpha$ and its downstream gene product CA9 were associated with methylation of genes involved in common cancer related biological pathways, could reflect a mechanism by which hypoxic tumors, behave more aggressively and eventually influence patient prognosis. The current data and in vitro studies by others ${ }^{22,23}$ make it tempting to speculate about the sequence of events during hypoxia in which epigenetic mechanisms such as promoter $\mathrm{CpG}$ island methylation may play a role.

Conflicting experimental results can be derived from the literature. An inverse relationship between the presence of extensive tumor hypoxia and the incidence of promoter CpG island methylation was shown in human colorectal cell lines, in which exposure to hypoxia for 24 hours induced a reduction of 15$20 \%$ in global promoter $\mathrm{CpG}$ island methylation ${ }^{24}$. In contrast, an altered 
cellular phenotype in response to chronic hypoxia was demonstrated, with significant increases in global levels of promoter $\mathrm{CpG}$ island methylation, and concomitant increased expression of DNA methyltransferase ${ }^{9}$. Although the effects of hypoxia on epigenetic changes apparently can occur in a bidirectional mode, with either decrease or increase in promoter $\mathrm{CpG}$ island methylation, these results nonetheless indicate that epigenetic changes may represent an additional level of cellular adaptation to hypoxia, in addition to the HIF induced cellular response.

In theory, three possible scenarios could be put forward to account for the relation between tumor hypoxia and epigenetic alterations: firstly, DNA promoter hypermethylation and silencing of relevant tumor suppressor or DNA repair genes, by interfering with their control functions, can lead to conditions of genomic instability, evasion of apoptosis and limitless replication, resulting in tumor growth and subsequent hypoxia. Conversely, hypoxia in a growing tumor mass could induce epigenetic changes, such as gene silencing through methylation. Lastly, promoter hypermethylation of specific genes could offer protection against the deleterious effects of hypoxia on tumor cells, thus rendering a selective growth advantage in tumors with the appropriate methylation status ${ }^{5,9}$.

Promoter $\mathrm{CpG}$ island methylation of the 16 genes we analyzed in the present study has also been detected in early lesions such as hyperplastic polyps and adenomas ${ }^{25-27}$, in which hypoxia does not play any significant role ${ }^{2}$, suggesting that these genes are already methylated before hypoxia has occurred, rather than methylation being a consequence of hypoxia. This is in line with the theory that by silencing of crucial cancer biology genes, tumors are able to adapt and behave more aggressively during hypoxia ${ }^{4,28}$. Strengthening this theory is the fact that within our series of CRCs, promoter CpG island methylation of CHFR and stromal expression of HIF-2 $\alpha$ and CA9 were both shown to be indicators of a more aggressive tumor behavior, since they were both related to a poorer patient survival (Chapters 4\&6). We concluded that expression of hypoxia regulated proteins HIF-2 $\alpha$ and CA9 in tumor associated stroma, as opposed to their expression in epithelial cancer cells, is associated with poorer outcome, suggesting that tumor hypoxia may influence tumor associated stromal cells in a way that ultimately effects patient prognosis. The results of the current study add to this observation, in that tumors with stromal expression of HIF-2 $\alpha$ and $\mathrm{CA} 9$, show promoter $\mathrm{CpG}$ island methylation of crucial cancer pathway genes occurs. As our study is correlative and descriptive in nature, and based on patient material, we are unable to pinpoint the precise mechanisms involved. There are however some indications from in vitro data, which have also shown that tumor stroma is involved in effecting tumor biology, e.g. by downregulation of caspases, crucial apoptosis mediators ${ }^{29-32}$. Interestingly, this downregulation was established by promoter $\mathrm{CpG}$ island methylation, as it was shown that the 
DNA-methylation inhibitor 5-azadeoxycytidine could reverse methylation and restore caspase expression ${ }^{33}$.

Apart from the discussion whether hypoxia should be considered a consequence or cause of epigenetic changes such as promoter $\mathrm{CpG}$ island methylation, the third aspect, protection of tumor cells against hypoxic cell damage, is also relevant. It has been shown that hypoxia, through induction of cell death, selects for cells with defective cell death regulators such as BNIP3 and TP53, thus evading apoptosis ${ }^{34}$. One way of achieving this situation is by promoter CpG island methylation and silencing of BNIP3. In the current study we found that BNIP3 methylated tumors more often showed CA9 epithelial expression (88\%), compared to BNIP3 unmethylated tumors $(73 \%), p=0.03$. Conceivably, the combination of BNIP3 methylation and CA9 epithelial expression reflects a synergistic mechanism to evade apoptosis, since CA9 expression maintains intracellular $\mathrm{pH}$, thereby preventing tumor cell apoptosis $^{35}$. This is in line with the concept that in hypoxic tumors there is a selective growth and survival advantage for cells with defects in the apoptotic machinery, by silencing of BNIP3 due to promoter CpG island methylation and TP53 mutations ${ }^{11,36}$. Epigenetic silencing of BNIP3 by promoter CpG island methylation has been reported in several cancer types and contributes to resistance to hypoxia-induced cell death ${ }^{37-39}$. The exact role of BNIP3 in CRC is unknown. In the next chapter we attempt to elucidate whether BNIP3 methylation and TP53 mutations in tumor cells, are related to patient outcome and apoptotic activity in hypoxic CRCs.

In summary, stromal expression of hypoxia regulated proteins HIF-2 $\alpha$ and CA9 was associated with promoter $\mathrm{CpG}$ island methylation of genes that participate in all of the major tumor biological pathways that were studied. Tumors with hypoxic features do not appear to show increased overall methylation, but rather harbour a selection of methylated genes, which could individually contribute to the more aggressive behaviour of these tumors. Further clinical and biological studies are necessary to validate our data and to unravel the precise mechanisms involved. 
Promoter $\mathrm{CpG}$ island methylation and hypoxia

\section{References}

1. Chiavarina B, Whitaker-Menezes D, Migneco G, et al: HIF1-alpha functions as a tumor promoter in cancer associated fibroblasts, and as a tumor suppressor in breast cancer cells: Autophagy drives compartment-specific oncogenesis. Cell Cycle 9:3534-51, 2010

2. Eltzschig HK, Carmeliet P: Hypoxia and inflammation. N Engl J Med 364:656-65, 2011

3. Franovic A, Holterman CE, Payette J, et al: Human cancers converge at the HIF-2alpha oncogenic axis. Proc Natl Acad Sci U S A 106:21306-11, 2009

4. Ruan K, Song G, Ouyang G: Role of hypoxia in the hallmarks of human cancer. J Cell Biochem 107:1053-62, 2009

5. Watson JA, Watson CJ, McCann A, et al: Epigenetics, the epicenter of the hypoxic response. Epigenetics 5:293-6, 2010

6. Herman JG, Latif $F$, Weng $Y$, et al: Silencing of the VHL tumor-suppressor gene by DNA methylation in renal carcinoma. Proc Natl Acad Sci U S A 91:9700-4, 1994

7. Fath DM, Kong $X$, Liang $D$, et al: Histone deacetylase inhibitors repress the transactivation potential of hypoxia-inducible factors independently of direct acetylation of HIF-alpha. J Biol Chem 281:13612-9, 2006

8. Beyer S, Kristensen MM, Jensen KS, et al: The histone demethylases JMJD1A and JMJD2B are transcriptional targets of hypoxia-inducible factor HIF. J Biol Chem 283:36542-52, 2008

9. Watson JA, Watson CJ, McCrohan AM, et al: Generation of an epigenetic signature by chronic hypoxia in prostate cells. Hum Mol Genet 18:3594-604, 2009

10. Bacon AL, Fox S, Turley $\mathrm{H}$, et al: Selective silencing of the hypoxia-inducible factor 1 target gene BNIP3 by histone deacetylation and methylation in colorectal cancer. Oncogene 26:132-41, 2007

11. An HJ, Lee H, Paik SG: Silencing of BNIP3 results from promoter methylation by DNA methyltransferase 1 induced by the mitogen-activated protein kinase pathway. Mol Cells 31:579-83, 2011

12. Bertholon J, Wang $\mathrm{Q}$, Falette $\mathrm{N}$, et al: Chfr inactivation is not associated to chromosomal instability in colon cancers. Oncogene 22:8956-60, 2003

13. Maddika S, Sy SM, Chen J: Functional Interaction between Chfr and Kif22 Controls Genomic Stability. J Biol Chem 284:12998-3003, 2009

14. Privette LM, Petty EM: CHFR: A Novel Mitotic Checkpoint Protein and Regulator of Tumorigenesis. Transl Oncol 1:57-64, 2008

15. Hanahan D, Weinberg RA: The hallmarks of cancer. Cell 100:57-70, 2000

16. Wiggers $\mathrm{T}$, Jeekel $\mathrm{J}$, Arends JW, et al: No-touch isolation technique in colon cancer: a controlled prospective trial. Br J Surg 75:409-15, 1988

17. Cleven $A H$, van Engeland $M$, Wouters $B G$, et al: Stromal expression of hypoxia regulated proteins is an adverse prognostic factor in colorectal carcinomas. Cell Oncol 29:229-40, 2007

18. Yoshimura H, Dhar DK, Kohno H, et al: Prognostic impact of hypoxia-inducible factors 1 alpha and 2alpha in colorectal cancer patients: correlation with tumor angiogenesis and cyclooxygenase-2 expression. Clin Cancer Res 10:8554-60, 2004

19. Herman JG, Graff JR, Myohanen S, et al: Methylation-specific PCR: a novel PCR assay for methylation status of CpG islands. Proc Natl Acad Sci U S A 93:9821-6, 1996

20. Derks S, Lentjes MH, Hellebrekers DM, et al: Methylation-specific PCR unraveled. Cell Oncol 26:291-9, 2004

21. Loannidis J: Why most published research findings are false. PLoS Med 2, 2005

22. Wang Z, Yang D, Zhang X, et al: Hypoxia-induced down-regulation of neprilysin by histone modification in mouse primary cortical and hippocampal neurons. PLoS One 6:e19229, 2011

23. Liu Q, Liu L, Zhao Y, et al: Hypoxia induces genomic DNA demethylation through the activation of HIF-1alpha and transcriptional upregulation of MAT2A in hepatoma cells. Mol Cancer Ther 10:1113-23, 2011

24. Shahrzad S, Bertrand K, Minhas K, et al: Induction of DNA hypomethylation by tumor hypoxia. Epigenetics 2:119-25, 2007 
$140 \mid$ Chapter 7

25. Derks S, Postma C, Moerkerk PT, et al: Promoter methylation precedes chromosomal alterations in colorectal cancer development. Cell Oncol 28:247-57, 2006

26. Kang GH: Four molecular subtypes of colorectal cancer and their precursor lesions. Arch Pathol Lab Med 135:698-703, 2011

27. Kim KM, Lee AJ, Ha A, et al: Molecular features of colorectal hyperplastic polyps and sessile serrated adenoma/polyp from Korea. American Journal of Surgical Pathology 35:1274-86, 2011

28. Pietras K, Ostman A: Hallmarks of cancer: interactions with the tumor stroma. Exp Cell Res 316:1324-31, 2010

29. Martinez-Outschoorn UE, Balliet RM, Rivadeneira DB, et al: Oxidative stress in cancer associated fibroblasts drives tumor-stroma co-evolution: A new paradigm for understanding tumor metabolism, the field effect and genomic instability in cancer cells. Cell Cycle 9:325676, 2010

30. Martinez-Outschoorn UE, Pavlides S, Whitaker-Menezes D, et al: Tumor cells induce the cancer associated fibroblast phenotype via caveolin-1 degradation: implications for breast cancer and DCIS therapy with autophagy inhibitors. Cell Cycle 9:2423-33, 2010

31. Martinez-Outschoorn UE, Trimmer C, Lin Z, et al: Autophagy in cancer associated fibroblasts promotes tumor cell survival: Role of hypoxia, HIF1 induction and NFkappaB activation in the tumor stromal microenvironment. Cell Cycle 9:3515-33, 2010

32. Martinez-Outschoorn UE, Whitaker-Menezes D, Pavlides S, et al: The autophagic tumor stroma model of cancer or "battery-operated tumor growth": A simple solution to the autophagy paradox. Cell Cycle 9:4297-306, 2010

33. Muerkoster SS, Werbing V, Koch D, et al: Role of myofibroblasts in innate chemoresistance of pancreatic carcinoma--epigenetic downregulation of caspases. Int J Cancer 123:1751-60, 2008

34. Graeber TG, Osmanian C, Jacks T, et al: Hypoxia-mediated selection of cells with diminished apoptotic potential in solid tumours. Nature 379:88-91, 1996

35. De Simone G, Supuran CT: Carbonic anhydrase IX: Biochemical and crystallographic characterization of a novel antitumor target. Biochim Biophys Acta 1804:404-9, 2010

36. Roberts AM, Watson IR, Evans AJ, et al: Suppression of hypoxia-inducible factor 2alpha restores p53 activity via Hdm2 and reverses chemoresistance of renal carcinoma cells. Cancer Res 69:9056-64, 2009

37. Abe T, Toyota M, Suzuki H, et al: Upregulation of BNIP3 by 5-aza-2'-deoxycytidine sensitizes pancreatic cancer cells to hypoxia-mediated cell death. J Gastroenterol 40:504-10, 2005

38. Yan J, Yun H, Yang Y, et al: Upregulation of BNIP3 promotes apoptosis of lung cancer cells that were induced by p53. Biochem Biophys Res Commun 346:501-7, 2006

39. Okami J, Simeone DM, Logsdon CD: Silencing of the hypoxia-inducible cell death protein BNIP3 in pancreatic cancer. Cancer Res 64:5338-46, 2004

40. Shima K, Morikawa T, Baba Y, et al: MGMT promoter methylation, loss of expression and prognosis in 855 colorectal cancers. Cancer Causes Control 22:301-9, 2011

41. Esteller M, Risques RA, Toyota M, et al: Promoter Hypermethylation of the DNA Repair Gene O6-Methyltransferase Is associated with the Presence of G:C to A:T Transition Mutations in p53 in Human Colorectal Tumorgenesis. Cancer research 61:4689-4692, 2001

42. Esteller M, Toyota M, Sanchez-Cespedes M, et al: Inactivation of the DNA repair gene O6methylguanine-DNA methyltransferase by promoter hypermethylation is associated with $\mathrm{G}$ to A mutations in K-ras in colorectal tumorigenesis. Cancer Res 60:2368-71, 2000

43. Herman JG, Umar A, Polyak K, et al: Incidence and functional consequences of hMLH1 promoter hypermethylation in colorectal carcinoma. Proc Natl Acad Sci U S A 95:6870-5, 1998

44. Fu Z, Regan K, Zhang L, et al: Deficiencies in Chfr and Mlh1 synergistically enhance tumor susceptibility in mice. J Clin Invest 119:2714-24, 2009

45. Brandes JC, van Engeland M, Wouters KA, et al: CHFR promoter hypermethylation in colon cancer correlates with the microsatellite instability phenotype. Carcinogenesis 26:1152-6, 2005 
46. Mizuno $\mathrm{K}$, Osada $\mathrm{H}$, Konishi $\mathrm{H}$, et al: Aberrant hypermethylation of the $\mathrm{CHFR}$ prophase checkpoint gene in human lung cancers. Oncogene 21:2328-33, 2002

47. Shibata $\mathrm{Y}$, Haruki N, Kuwabara $\mathrm{Y}$, et al: Chfr expression is downregulated by $\mathrm{CpG}$ island hypermethylation in esophageal cancer. Carcinogenesis 23:1695-9, 2002

48. Corn PG, Summers MK, Fogt F, et al: Frequent hypermethylation of the $5^{\prime} \mathrm{CpG}$ island of the mitotic stress checkpoint gene Chfr in colorectal and non-small cell lung cancer. Carcinogenesis 24:47-51, 2003

49. Satoh A, Toyota $M$, Itoh $F$, et al: Epigenetic inactivation of CHFR and sensitivity to microtubule inhibitors in gastric cancer. Cancer Res 63:8606-13, 2003

50. Kang D, Chen J, Wong J, et al: The checkpoint protein Chfr is a ligase that ubiquitinates Plk1 and inhibits Cdc2 at the G2 to M transition. J Cell Biol 156:249-59, 2002

51. Scolnick DM, Halazonetis TD: Chfr defines a mitotic stress checkpoint that delays entry into metaphase. Nature 406:430-5, 2000

52. Moinova HR, Chen WD, Shen L, et al: HLTF gene silencing in human colon cancer. Proc Natl Acad Sci U S A 99:4562-7, 2002

53. Motegi A, Liaw HJ, Lee KY, et al: Polyubiquitination of proliferating cell nuclear antigen by HLTF and SHPRH prevents genomic instability from stalled replication forks. Proc Natl Acad Sci U S A 105:12411-6, 2008

54. Donninger $\mathrm{H}$, Allen $\mathrm{N}$, Henson $\mathrm{A}$, et al: Salvador is a tumor suppressor effector of RASSF1A with Hippo pathway independent functions. J Biol Chem, 2011

55. Donninger H, Vos MD, Clark GJ: The RASSF1A tumor suppressor. J Cell Sci 120:3163-72, 2007

56. Ahlquist $\mathrm{T}$, Bottillo I, Danielsen $\mathrm{SA}$, et al: RAS signaling in colorectal carcinomas through alteration of RAS, RAF, NF1, and/or RASSF1A. Neoplasia 10:680-6, 2 p following 686, 2008

57. Robertson KD, Jones PA: Tissue-specific alternative splicing in the human INK4a/ARF cell cycle regulatory locus. Oncogene 18:3810-20, 1999

58. Agrawal A, Yang J, Murphy RF, et al: Regulation of the p14ARF-Mdm2-p53 pathway: an overview in breast cancer. Exp Mol Pathol 81:115-22, 2006

59. Serrano M, Gomez-Lahoz E, DePinho RA, et al: Inhibition of ras-induced proliferation and cellular transformation by p16INK4. Science 267:249-52, 1995

60. Herman JG, Merlo A, Mao L, et al: Inactivation of the CDKN2/p16/MTS1 gene is frequently associated with aberrant DNA methylation in all common human cancers. Cancer Res 55:4525-30, 1995

61. Sagane K, Ohya $Y$, Hasegawa $Y$, et al: Metalloproteinase-like, disintegrin-like, cysteine-rich proteins MDC2 and MDC3: novel human cellular disintegrins highly expressed in the brain. Biochem J 334 ( Pt 1):93-8, 1998

62. Hamilton SR, A.Aaltonen L: Pathology \& genetics of Tumours of the Digestive System, in Kleihues P, Sobin LH (eds): World Health Organization of the Digestive System. Lyon, France, International Agency for Research on Cancer, 2000

63. Hu C, Lv H, Pan G, et al: The expression of ADAM23 and its correlation with promoter methylation in non-small-cell lung carcinoma. Int J Exp Pathol, 2011

64. Costa FF, Verbisck NV, Salim AC, et al: Epigenetic silencing of the adhesion molecule ADAM23 is highly frequent in breast tumors. Oncogene 23:1481-8, 2004

65. Choi JS, Kim KH, Jeon YK, et al: Promoter hypermethylation of the ADAM23 gene in colorectal cancer cell lines and cancer tissues. Int J Cancer 124:1258-62, 2009

66. Sprecher CA, Kisiel W, Mathewes S, et al: Molecular cloning, expression, and partial characterization of a second human tissue-factor-pathway inhibitor. Proc Natl Acad Sci U S A 91:3353-7, 1994

67. Glockner SC, Dhir M, Yi JM, et al: Methylation of TFPI2 in stool DNA: a potential novel biomarker for the detection of colorectal cancer. Cancer Res 69:4691-9, 2009

68. Kim YH, Lee HC, Kim SY, et al: Epigenomic Analysis of Aberrantly Methylated Genes in Colorectal Cancer Identifies Genes Commonly Affected by Epigenetic Alterations. Ann Surg Oncol, 2011

69. Kinzler KW, Nilbert MC, Su LK, et al: Identification of FAP locus genes from chromosome 5q21. Science 253:661-5, 1991 
70. Vogelstein B, Bearon ER, Hamilton SR: Genetic alterations during colorectal-tumor development. New England Journal of Medicine 319:525-32, 1988

71. $\mathrm{Fu} \mathrm{X,} \mathrm{Li} \mathrm{J,} \mathrm{Li} \mathrm{K,} \mathrm{et} \mathrm{al:} \mathrm{Hypermethylation} \mathrm{of} \mathrm{APC} \mathrm{promoter} 1 \mathrm{~A}$ is associated with moderate activation of Wnt signalling pathway in a subset of colorectal serrated adenomas. Histopathology 55:554-63, 2009

72. Croker BA, Kiu H, Nicholson SE: SOCS regulation of the JAK/STAT signalling pathway. Semin Cell Dev Biol 19:414-22, 2008

73. Kile BT, Viney EM, Willson TA, et al: Cloning and characterization of the genes encoding the ankyrin repeat and SOCS box-containing proteins Asb-1, Asb-2, Asb-3 and Asb-4. Gene 258:31-41, 2000

74. Wong PK, Egan PJ, Croker BA, et al: SOCS-3 negatively regulates innate and adaptive immune mechanisms in acute IL-1-dependent inflammatory arthritis. J Clin Invest 116:157181,2006

75. Li QL, Ito K, Sakakura C, et al: Causal relationship between the loss of RUNX3 expression and gastric cancer. Cell 109:113-24, 2002

76. Kaio E, Tanaka S, Oka S, et al: Clinical significance of thrombospondin-1 expression in relation to vascular endothelial growth factor and interleukin-10 expression at the deepest invasive tumor site of advanced colorectal carcinoma. Int J Oncol 23:901-11, 2003

77. Toyota M, Ohe-Toyota M, Ahuja N, et al: Distinct genetic profiles in colorectal tumors with or without the CpG island methylator phenotype. Proc Natl Acad Sci U S A 97:710-5, 2000

78. Shibata D, Mori $\mathrm{Y}$, Cai K, et al: RAB32 hypermethylation and microsatellite instability in gastric and endometrial adenocarcinomas. Int J Cancer 119:801-6, 2006

79. Mori Y, Yin J, Sato F, et al: Identification of genes uniquely involved in frequent microsatellite instability colon carcinogenesis by expression profiling combined with epigenetic scanning. Cancer Res 64:2434-8, 2004

80. Bao X, Faris $\mathrm{AE}$, Jang EK, et al: Molecular cloning, bacterial expression and properties of Rab31 and Rab32. Eur J Biochem 269:259-71, 2002

81. Teider N, Scott DK, Neiss A, et al: Neuralized1 causes apoptosis and downregulates Notch target genes in medulloblastoma. Neuro Oncol 12:1244-56, 2010

82. Nakamura $\mathrm{H}$, Yoshida M, Tsuiki $\mathrm{H}$, et al: Identification of a human homolog of the Drosophila neuralized gene within the 10q25.1 malignant astrocytoma deletion region. Oncogene 16:1009-19, 1998

83. Subramaniam MM, Chan JY, Soong $R$, et al: RUNX3 inactivation by frequent promoter hypermethylation and protein mislocalization constitute an early event in breast cancer progression. Breast Cancer Res Treat 113:113-21, 2009

84. Subramaniam MM, Chan JY, Soong R, et al: RUNX3 inactivation in colorectal polyps arising through different pathways of colonic carcinogenesis. Am J Gastroenterol 104:426-36, 2009

85. Subramaniam MM, Chan JY, Yeoh KG, et al: Molecular pathology of RUNX3 in human carcinogenesis. Biochim Biophys Acta 1796:315-31, 2009

86. Kodach LL, Jacobs RJ, Heijmans J, et al: The role of EZH2 and DNA methylation in the silencing of the tumour suppressor RUNX3 in colorectal cancer. Carcinogenesis 31:1567-75, 2010

87. Lee H, Paik SG: Regulation of BNIP3 in normal and cancer cells. Mol Cells 21:1-6, 2006

88. Vande Velde C, Cizeau J, Dubik D, et al: BNIP3 and genetic control of necrosis-like cell death through the mitochondrial permeability transition pore. Mol Cell Biol 20:5454-68, 2000

89. Ray R, Chen G, Vande Velde $\mathrm{C}$, et al: BNIP3 heterodimerizes with $\mathrm{Bcl}-2 / \mathrm{Bcl}-\mathrm{X}(\mathrm{L})$ and induces cell death independent of a Bcl-2 homology $3(\mathrm{BH} 3)$ domain at both mitochondrial and nonmitochondrial sites. J Biol Chem 275:1439-48, 2000

90. Mellor HR, Harris AL: The role of the hypoxia-inducible BH3-only proteins BNIP3 and BNIP3L in cancer. Cancer Metastasis Rev 26:553-66, 2007

91. Murai M, Toyota M, Suzuki $\mathrm{H}$, et al: Aberrant methylation and silencing of the BNIP3 gene in colorectal and gastric cancer. Clin Cancer Res 11:1021-7, 2005 


\title{
Chapter
}

Poorer outcome in stromal HIF-2 $\alpha$ and

CA9 positive colorectal adenocarcinomas is associated with Wild-type TP53 but not with BNIP3 promoter hypermethylation or apoptosis

\author{
AHG Cleven \\ BG Wouters \\ B Schutte \\ AJG Spiertz \\ $M$ van Engeland \\ AP de Bruïne
}

British Journal of Cancer 2008;99:727-733 


\section{Abstract}

Stromal expression of hypoxia inducible factor $2 \alpha$ (HIF-2 $\alpha$ ) and Carbonic Anhydrase 9 (CA9) are associated with a poorer prognosis in colorectal cancer (CRC). Tumor cell death, regulated by a hypoxic stromal microenvironment could be of importance in this respect. Therefore, we correlated apoptosis, TP53 mutational status and BNIP3 promoter hypermethylation of CRC cells with HIF-2 $\alpha$ and CA9 related poor outcome.

In a series of 195 CRCs, TP53 mutations in exons 5-8 were analyzed by direct sequencing and promoter hypermethylation of BNIP3 was determined by methylation specific PCR (MSP). Expression of HIF-2 $\alpha, \mathrm{CA} 9, \mathrm{p} 53, \mathrm{BNIP} 3$ and M30 were analyzed immunohistochemically.

Poorer survival of HIF-2 $\alpha$ and CA9 stromal positive CRCs was associated with wild-type TP53 $(p=0.001$ and $p=0.0391)$, but not with BNIP3 methylation. Furthermore, apoptotic levels were independent of TP53 status, but lower in unmethylated BNIP3 CRCs $(p=0.004)$.

It appears that wild-type TP53 in CRC cells favours progression of tumors expressing markers for hypoxia in their stroma, rather than in the epithelial compartment. Preserved BNIP3 function in $\mathrm{CRC}$ cells lowers apoptosis, and may thus be involved in alternative cell death pathways, such as autophagic cell death. However, BNIP3 silencing in tumor cells does not impact on hypoxia driven poorer prognosis.

These results suggest that the biology of CRC cells can be modified by alterations in the tumor microenvironment under conditions of tumor hypoxia. 
TP53, BNIP3 and poor outcome in HIF-2 $\alpha$ and CA9 positive CRCs $\mid{ }_{145}$

\section{Introduction}

Hypoxia has been reported to influence tumor biology in opposing ways. It can directly induce cell death by activating apoptosis or autophagy, yet hypoxic zones in solid tumors also harbour viable cells resistant to treatment which contribute to poor patient outcome ${ }^{1}$. Hypoxia influences the expression of genes involved in cell death and energy homeostasis mainly by stabilization and activation of the Hypoxia Inducible Factor (HIF) family of transcription factors, influencing angiogenesis (VEGF), glycolysis (GLUT1), $\mathrm{pH}$ regulation (CA9), apoptosis (TP53, BNIP3) and autophagy (BNIP3) ${ }^{2,3}$.

A common hallmark of solid tumors under hypoxic stress is an increased ATP requirement, which is supplied by induction of anaerobic glycolysis. This subsequently leads to a high production of intracellular lactate, requiring regulation of intracellular $\mathrm{pH}$, a process mediated partly by HIF dependent upregulation of Carbonic Anhydrase 9 (CA9). CA9 catalyzes the extracellular trapping of acid by hydrating cell-generated $\mathrm{CO}_{2}$ into $\mathrm{HCO}_{3}^{-}$and $\mathrm{H}^{+4}$. Consequently, CA9 expression may serve as a marker for metabolic adaptation during hypoxia.

Upregulation of p53 and BNIP3 proteins, which stimulate cell death via apoptosis and/or autophagy, appears contradictory to the adverse association between tumor hypoxia and prognosis. However, it was suggested that through induction of cell death, hypoxia selects for cells with defective cell death regulators such as TP53 $3^{5}$. In non-selected cells hypoxia can induce expression of p53 and activate p53 mediated $G_{0} / G_{1}$ arrest or apoptosis, although secondary effects such as extracellular acidosis and glucose deprivation are necessary for p53 mediated apoptosis ${ }^{5-8}$. Over $50 \%$ of human tumors contain somatic mutations in the TP53 gene, resulting in a defective apoptotic response $^{9,10}$. Therefore, TP53 mutations are expected to decrease the susceptibility of tumor cells to hypoxia induced cell death, as shown in vitro ${ }^{5,11}$. $B N I P 3$ is a Bcl-2 family member, containing a single BcL-2 homology $3(\mathrm{BH} 3)$ domain and a transmembrane domain localizing it to the outer mitochondrial membrane ${ }^{12,13}$. It is activated by HIF during hypoxia and initiates programmed cell death through apoptosis or autophagy ${ }^{14,15}$. Epigenetic silencing of BNIP3 by promoter hypermethylation has been reported in several cancer types and contributes to resistance to hypoxia-induced cell death ${ }^{16-18}$. The role of BNIP3 in CRC is unknown, although $66 \%$ of tumors show BNIP3 silencing by promoter hypermethylation ${ }^{19,20}$.

Usually, the effects of hypoxia in solid tumors are studied within the tumor cells themselves, neglecting the hypoxic response in tumor associated stroma. In a previous study we found that hypoxia within the tumor associated stroma is indeed correlated with a poorer outcome in patients with colorectal cancer who are treated by surgery alone. In a multivariate model, stromal expression of 
HIF-2 $\alpha$ and CA9 both were independent adverse prognostic factors, whereas HIF-1 $\alpha$ was not. Furthermore, expression of hypoxia related proteins HIF-1 $\alpha$, GLUT1 and CA9 in the tumor cells self was not associated with poorer patient survival $^{21}$.

Our previous findings indicate that hypoxic conditions may modulate the tumor stroma in such a way, that a more aggressive tumor behavior is facilitated, ultimately leading to decreased patient survival.

The present study attempts to elucidate whether changes in the epithelial cell compartment of CRC, such as apoptosis and concomitant (epi-)genetic changes that are confined to the tumor cells, are related to hypoxia related changes in the stromal compartment. For this purpose, we correlated alterations of TP53 and BNIP3 in tumor cells with expression of hypoxia related proteins HIF-2 $\alpha$ and CA9 in relation with patient outcome and apoptotic activity in CRCs.

\section{Patients and methods}

\section{Patient population}

Patients were entered in two multi-center prospective clinical trials in the Netherlands, between 1979 and 1981. One trial was designed to compare patient survival after treatment of colon cancer by conventional surgery or the no-touch isolation technique ${ }^{22}$. The second trial was conducted to compare survival in rectal cancer patients with or without preoperative radiotherapy. In the current study, we included only the patients who did not undergo preoperative radiotherapy. At the time the trial was conducted, only surgical removal of the tumors was performed, and adjuvant chemotherapy was not yet standard practice. This study population therefore enables unbiased study of the influence of hypoxic conditions on tumor biology.

Tumor tissues were fixed in buffered formalin, sectioned, and embedded in paraffin. Experienced pathologists documented the histopathological characteristics of the tumors (Table 8.1). Follow up took place every 3 months during the first three years and every 6 months between three and five years after initial diagnosis and surgery. Standard protocols were followed, with routine blood counts and chemistry studies (including CEA levels) at each visit and liver ultrasound, chest $x$-ray and colonoscopy annually, to evaluate recurrence of disease and disease-related death. After five year follow up period, only time and cause of death were registered. Follow-up was complete for all patients. Failure was defined as death due to recurrent disease, excluding postoperative mortality within 30 days and non-disease related death. 
For immunohistochemical and molecular analysis, tumor tissues from 195 CRC patients were available. The distribution of age, gender, tumor stage, location and type of tumor, frequency of events and mean follow-up time of the patients in this study are representative for the patients in the trial (see Table 8.1).

Table 8.1 TP53 mutation analyses, BNIP3 methylation and clinicopathological characteristics.

\begin{tabular}{|c|c|c|c|c|c|c|c|c|c|c|}
\hline & \multicolumn{4}{|c|}{ TP53 } & \multicolumn{6}{|c|}{ BNIP3 } \\
\hline & \multicolumn{2}{|c|}{ Wt } & \multicolumn{2}{|c|}{ Mutant } & \multirow[b]{2}{*}{$\mathrm{P}$} & \multicolumn{2}{|c|}{$\mathbf{U}$} & \multicolumn{2}{|c|}{$\mathbf{M}$} & \multirow[b]{2}{*}{$\mathrm{P}$} \\
\hline & No & $\%$ & No & $\%$ & & No & $\%$ & No & $\%$ & \\
\hline Total patients & 78 & (50) & 77 & (50) & & 92 & (47) & 103 & (53) & \\
\hline Age & & & & & 297 & & & & & .759 \\
\hline$<69$ years & 35 & (45) & 41 & (53) & & 44 & (48) & 47 & (46) & \\
\hline$>69$ years & 43 & (55) & 36 & (47) & & 48 & (52) & 56 & (54) & \\
\hline Sex & & & & & .942 & & & & & .788 \\
\hline Male & 35 & (45) & 35 & (45) & & 42 & (46) & 49 & (48) & \\
\hline Female & 43 & (55) & 42 & (55) & & 50 & (54) & 54 & (52) & \\
\hline Tumor location & & & & & 202 & & & & & .016 \\
\hline Proximal & 32 & (41) & 24 & (31) & & 25 & (27) & 45 & (44) & \\
\hline Distal & 46 & (59) & 53 & (69) & & 67 & (73) & 58 & (56) & \\
\hline Tumor & & & & & .819 & & & & & .967 \\
\hline Colon & 49 & (63) & 47 & (61) & & 61 & (66) & 68 & (66) & \\
\hline Rectum & 29 & (37) & 30 & (39) & & 31 & (34) & 35 & (34) & \\
\hline Differentiation & & & & & .913 & & & & & .959 \\
\hline Well & 8 & (10) & 8 & (10) & & 10 & (11) & 11 & (11) & \\
\hline Moderate/poor & 70 & (90) & 69 & (90) & & 82 & (89) & 92 & (89) & \\
\hline TNM & & & & & .471 & & & & & .001 \\
\hline 1 & 1 & (1) & 2 & (2) & & 3 & (4) & 1 & (1) & \\
\hline 2 & 40 & (51) & 46 & (60) & & 61 & (66) & 55 & (53) & \\
\hline 3 & 28 & (36) & 19 & (25) & & 14 & (15) & 40 & (39) & \\
\hline 4 & 9 & (12) & 10 & (13) & & 14 & (15) & 7 & (7) & \\
\hline
\end{tabular}

Wt, Wild-type; U, Unmethylated; M, Methylated. Location: proximal or distal to splenic flexure.

\section{Genomic DNA Isolation}

Genomic DNA was extracted from CRC tissues using PureGene ${ }^{\mathrm{TM}}$ Genomic DNA Isolation Kit (Gentra Systems) based on the manufacturer's protocol.

\section{TP53 sequencing}

Mutation analyses of TP53 exons 5-8 was performed using a semi-nested PCR approach, (see supplemental Table 8.1S for primer sequences). CaCo2 (exon 6, codon 204 non-sense mutation) was included as a control. Direct sequencing of PCR products was performed using the BigDye ${ }^{\circledR}$ Terminator v1.1 Cycle Sequencing Kit (Applied Biosystems) and analysed on the ABI 3730 DNA Analyzer (Applied Biosystems). Mutation detection was performed using Mutation Surveyor DNA Variant Analysis Software v3.0 (SoftGenetics LLC, USA). The results of the mutation analyses are listed in Table 8.2. 
$148 \mid$ Chapter 8

Furthermore, we assessed whether TP53 missense-mutants were transcriptionally active or not, based on the IARC prediction models http://www.p53.iarc.fr/MutationValidationCriteria.asp). Missense mutations were classified as either transactivation incompetent or transactivation competent missense mutations.

Table 8.2 Frequency and type of TP53 alterations.

\begin{tabular}{lcc}
\hline TP53 Mutation & No & $\%$ \\
\hline Wild type & 72 & $(46)$ \\
Tr-Incom. Missense & 57 & $(37)$ \\
Tr-Com. Missense & 8 & $(5)$ \\
Silent & 4 & $(3)$ \\
Non-sense & 5 & $(3)$ \\
Deletion & 4 & $(3)$ \\
Insertion & 3 & $(2)$ \\
Polymorphism & 2 & $(1)$ \\
\hline Total & 155 & $(100)$ \\
\hline
\end{tabular}

Tr-Incom, Transactivation incompetent; miss-mutations, missense mutation;

Tr-Com, Transactivation competent.

\section{BNIP3 Promoter methylation analysis}

$B N I P 3$ promoter methylation was determined by sodium bisulfite modification of genomic DNA using the EZ DNA methylation kit (ZYMO research Co., Orange, CA). Methylation Specific PCR (MSP) was performed as described in detail elsewhere ${ }^{23,24}$. DNA was first amplified with BNIP3 flanking PCR primers that amplify bisulfite modified DNA but do not preferentially amplify methylated or unmethylated DNA. The resulting template was used as the template for BNIP3 MSP. For primer sequences see supplemental Table 8.1S. All PCRs were performed with a control for unmethylated BNIP3 alleles (normal lymphocyte DNA) and a positive control for methylated BNIP3 alleles (Sssl methyltransferase (New England Biolabs) treated normal lymphocyte DNA) and a negative control without DNA. Each PCR reaction was loaded onto a $2 \%$ agarose gel, stained with Gelstar $^{\circledR}$ (Cambrex Bioscience Rockland Inc, USA) and visualized under UV illumination.

\section{Immunohistochemistry}

Serial formalin fixed, paraffin-embedded tissues sections $(4 \mu \mathrm{m})$ were stained for HIF-2 $\alpha$ and CA9 as described previously ${ }^{21}$.

Briefly, HIF-2 $\alpha$ staining: Antigen retrieval was performed by microwave treatment $(750 \mathrm{~W}$ for $20 \mathrm{~min}$ in $1 \mathrm{mM} \mathrm{TE}$ buffer $\mathrm{pH} 8.0$ ), followed by $30 \mathrm{~min}$. cooling in buffer. Slides were blocked in $25 \%$ normal serum for $10 \mathrm{~min}$. 
Sections were incubated with primary antibody HIF-2 $\alpha$ (1:500) for 100 min. (anti-HIF2 alpha monoclonal: ab8365 AbCam, UK).

CA9 staining: Slides were blocked in $25 \%$ normal serum for $10 \mathrm{~min}$., then incubated for 45 min. with primary CA9 antibody MoAb M75 (1:50) (anti-human CA9, kindly supplied by Dr. S. Pastorekova) at room temperature. In addition, serial sections were stained for p53, BNIP3 and M30, as follows: p53 staining: Antigen retrieval was performed by microwave treatment $(750 \mathrm{~W}$ for $15 \mathrm{~min}$. in Antigen Retrieval (Dako)), followed by $30 \mathrm{~min}$. cooling in buffer. Slides were blocked in $25 \%$ normal serum for 10 min. Sections were incubated for $45 \mathrm{~min}$ at room temperature with primary antibody p53 (1:500) (anti-p53 monoclonal (DO-7), M7001 DAKO, Denmark).

BNIP3 staining: Antigen retrieval was performed by microwave treatment (750 W for $15 \mathrm{~min}$. in Antigen Retrieval (Dako)), followed by $30 \mathrm{~min}$. cooling in buffer. Slides were blocked in $25 \%$ normal serum for 10 min. Sections were incubated for $180 \mathrm{~min}$ at room temperature with primary antibody BNIP3 (1:400) (anti-BNIP3 monoclonal (Ana40): ab10433, UK).

M30 staining: Antigen retrieval was performed by microwave treatment $(750 \mathrm{~W}$ for $10 \mathrm{~min}$. in Antigen Retrieval (Dako)), followed by $30 \mathrm{~min}$. cooling in buffer. Slides were blocked in $25 \%$ normal serum for $10 \mathrm{~min}$. Sections were incubated for $45 \mathrm{~min}$ at room temperature with primary Cytodeath antibody M30 (1:50) (mouse monoclonal (CloneM30), Roche Applied Science, Germany).

Each staining protocol was started with pre-incubating in $0.6 \%$ hydrogen peroxide for $20 \mathrm{~min}$ to block endogenous peroxidase activity. Furthermore, as a negative control, TBS buffer instead of primary antibody was used. Visualization was performed using Dako Envision, Peroxidase, mouse System (K4001, DAKO, Denmark). Powerenvison Poly-HRP (50510-60307, Immunologic, the Netherlands) was used for M30 visualization. The slides were counterstained with hematoxylin.

\section{Evaluation of $\mathrm{IHC}$}

Evaluation for HIF-2 $\alpha$ and CA9 staining as described previously in detail ${ }^{21}$. Briefly, localisation (epithelial or stromal) was scored separately. For the category stromal staining only the stromal myofibroblasts were taken into account, not the tumor infiltrating inflammatory cells or the lamina propia of the normal mucosa. If nuclear staining was present in $>5 \%$ of the tumor epithelial cells or stromal cells, the sample was considered positive for HIF-2 $\alpha$.

If membranous staining occurred in $>5 \%$ of the tumor epithelial cells or stromal cells, samples were considered positive for $\mathrm{CA} 9^{25}$.

TP53 and BNIP3 staining were considered positive by the presence of nuclear staining for TP53 and cytoplasmic staining for BNIP3 respectively, in $>5 \%$ of tumor cells. 
$150 \mid$ Chapter 8

M30 expression was documented as the number of positive M30 cells per square millimeter of tumor cells (counted in 10 high-power fields $(\times 100)$ per tumor) (Figure 8.1A, B). Apoptosis was categorized as Low apoptosis when the number of M30 positive cells $\leq 10$ (mean), and High apoptosis when the number of M30 positive cells $>10^{26,27}$.

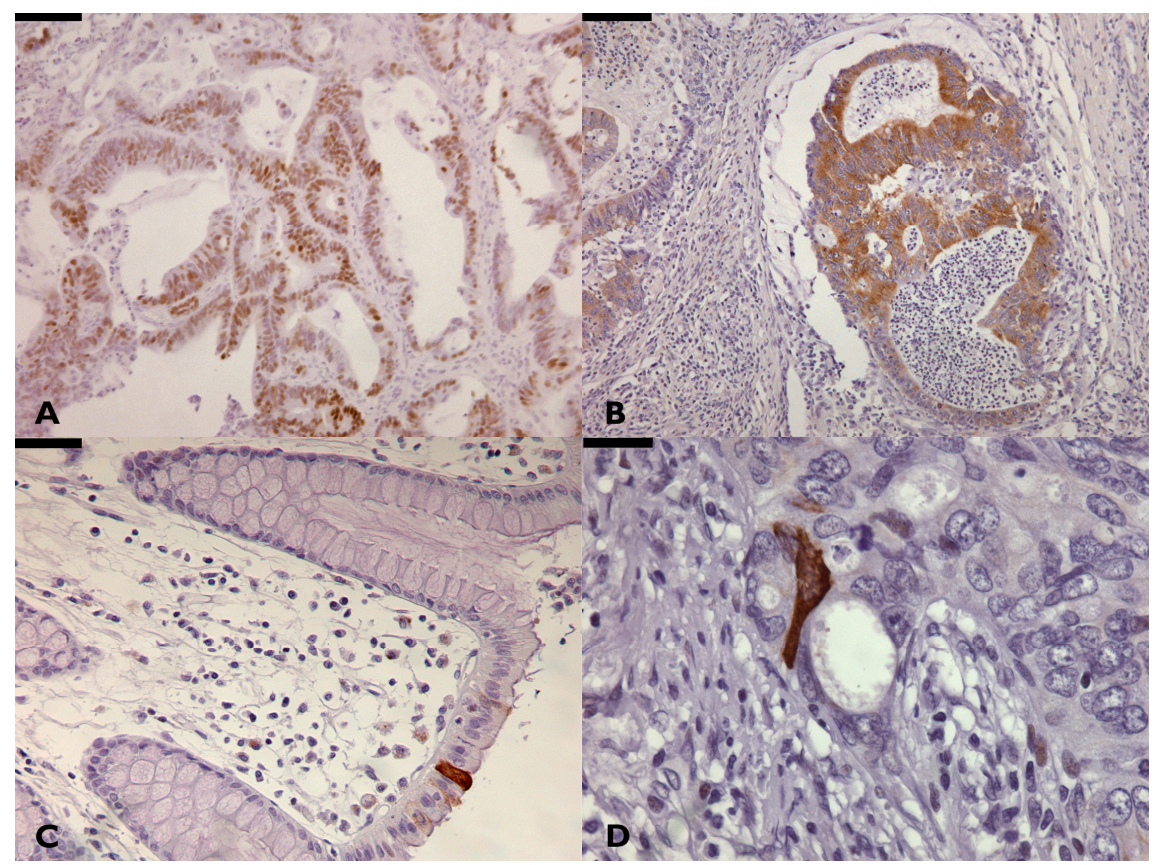

Figure 8.1 (A) Nucleair expression of TP53 in colorectal tumor epithelial cells. (B) Cytoplasmic expression of BNIP3 in colorectal tumor epithelial cells. (C,D) Expression of M30 in colorectal tissue. (C) Expression of M30 in normal colon tissue. (D) Expression of M30 in tumor epithelial cell. $(A, B)$ Original magnifications $\times 20 . \quad(C-D)$ Original magnifications $\times 40$. Black bar pictures $A, B=200 \mu \mathrm{m}, C, D=100 \mu \mathrm{m}$.

\section{Data-analysis}

Correlations between HIF-2a, CA9, BNIP3, TP53, M30 and clinicopathological parameters were determined by the Pearson Chi-Square and Fisher's exact test where appropriate. To evaluate the relationship between HIF-2 $\alpha, B N I P 3$, TP53 and survival, Kaplan-Meier survival curves were calculated. Differences between groups were determined by using the Log-rank test. The endpoint for analyses was overall survival starting from the day of surgery. All p-values are two sided and $p$-values $<0.05$ were considered statistically significant. Correction for multiple comparisons was performed using the Bonferroni 
procedure. Patients with unknown and unspecified scores have been omitted from analyses for that specific variable. SPSS 12.0 software was used for data analyses.

\section{Results}

\section{TP53 mutations}

TP53 mutation analysis was successful in 155/195 (79\%) of CRCs. CRCs were classified as having no TP53 mutations 72/155 (46\%), silent mutations 4/155 (3\%) or known common polymorphism (exon 6, codon 213, CGA>CGG, R/R, refSNP rs1800372) 2/155 (1\%) (Table 8.2). 37\% (57/155) of CRCs were classified as transactivation incompetent missense mutations and 5\% (8/155) of CRCs were classified as transactivation competent missense mutations. Correlations between TP53 and other variables did not change with respect to the predicted presence or absence of transcriptional activity of TP53 mutants. Therefore, in further analyses CRCs were classified as TP53 wild-type when no mutations, silent mutations or a known common polymorphism were found, and as mutant TP53 when CRCs had either missense, nonsense or frame shift mutations. Using this classification, 77/155 (50\%) of CRC's showed TP53 mutations (Table 8.1), which is in agreement with data published by others ${ }^{28-37}$. p53 protein expression was only observed in the nucleus of epithelial cells (Figure 8.1A). There was a significant correlation between the absence of p53 protein expression and wild-type TP53 versus the presence of p53 protein in mutant TP53, $\mathrm{p}=0.029$ (data not shown). No correlation between TP53 mutation status and clinicopathological data was observed (Table 8.1).

\section{TP53 mutations, patient survival and apoptosis}

Overall no significant survival difference between TP53 wild-type and mutant CRCs was observed (data not shown). However, the previously reported association between HIF-2 $\alpha$ or CA9 positive CRCs and poor prognosis was found to exist exclusively in TP53 wild-type CRCs $(p=0.001$ and $p=0.0829$ respectively, Figure $8.2 \mathrm{~A}+\mathrm{B}$ ). Furthermore, there was a significant difference in survival between stromal CA9 expression (38\% 5-years survival) versus epithelial CA9 expression (71\% 5-years survival) within TP53 wild-type tumors, $\mathrm{p}=0.0391$ (data not shown). Overall levels of HIF-2 $\alpha$ or CA9 expression were not different between wild-type and mutant TP53 CRCs (Data not shown). Survival of TP53 mutant CRCs was not related to HIF-2 $\alpha$ nor CA9 expression $(p=0.9312$ and $p=0.8456$, respectively, Figure $8.2 E+F)$. These data suggest that wild-type TP53 CRCs are less susceptible to the adverse effects of hypoxia. Since TP53 can induce apoptosis during hypoxia, we assessed the extent of apoptosis (M30 staining, Figure $8.1 \mathrm{C}+\mathrm{D}$ ). Overall we found no 
differences in apoptotic levels between TP53 wild-type and TP53 mutant CRCs (Table 8.3), or between HIF-2 $\alpha$ and CA9 positive or negative CRCs (data not shown). This was found regardless of TP53 mutation status. These results indicate that presence or absence of functional p53 protein is not decisive for determining the extent of apoptosis in CRCs.

\section{BNIP3 methylation}

BNIP3 promoter methylation analysis was successful in 195/195 CRC's $(100 \%)$. Overall $53 \% \quad(n=103 / 195)$ of CRCs showed BNIP3 promoter hypermethylation (Table 8.1), which is in agreement with data published by others $^{12,20}$.

The relationship between protein expression of BNIP3 and BNIP3 promoter methylation status was analyzed in a randomly selected subset of patients $(n=31)$. BNIP3 protein expression was only observed in the cytoplasm of epithelial cells (Figure 8.1B). BNIP3 promoter methylated CRCs less frequently demonstrated BNIP3 protein expression than unmethylated CRCs $(25 \%$ vs. $75 \%$, respectively).

\section{BNIP3 methylation, patient survival and apoptosis}

Overall there was no significant survival difference between BNIP3 methylated and unmethylated CRCs (data not shown). Although in HIF-2a stromal negative CRCs, BNIP3 methylation occurred in 61\% (33/54), and did not influence prognosis whereas in the HIF-2 $\alpha$ positive tumors methylation was observed in almost equal frequency, $52 \%$ (68/132) but was associated with poorer patient survival ( $p=0.006$, Figure $8.2 \mathrm{G}$ ). Similarly, exclusively stromal (and not epithelial) expression of CA9 was an indicator of a poorer prognosis in both $B N I P 3$ methylated and BNIP3 unmethylated tumors $(p=0.0495$ and $p=0.0725$, respectively, Figure $8.2 \mathrm{D}, \mathrm{H}$ ).

This suggests that hypoxic CRCs with stromal expression of HIF-2 $\alpha$ and CA9 have a poorer prognosis, independent of BNIP3 methylation.

Since BNIP3 has been reported to induce apoptosis in response to hypoxia, its methylation and associated down regulation might be expected to result in less apoptosis in the HIF-2a subgroup. However, a low apoptotic activity (low M30 expression) was detected more frequently in BNIP3 unmethylated CRCs $68 \%$ (50/73) compared to BNIP3 methylated CRCs $46 \%$ (42/91, $\mathrm{p}=0.004$, Table 8.3). Although tumors with both methylated BNIP3 and stromal HIF-2a expression showed a poorer patient survival compared to HIF-2a negative tumors, this was not related to apoptosis (data not shown). Furthermore, we did not detect differences in apoptotic levels between tumors with or without CA9 expression, regardless of BNIP3 methylation status. 

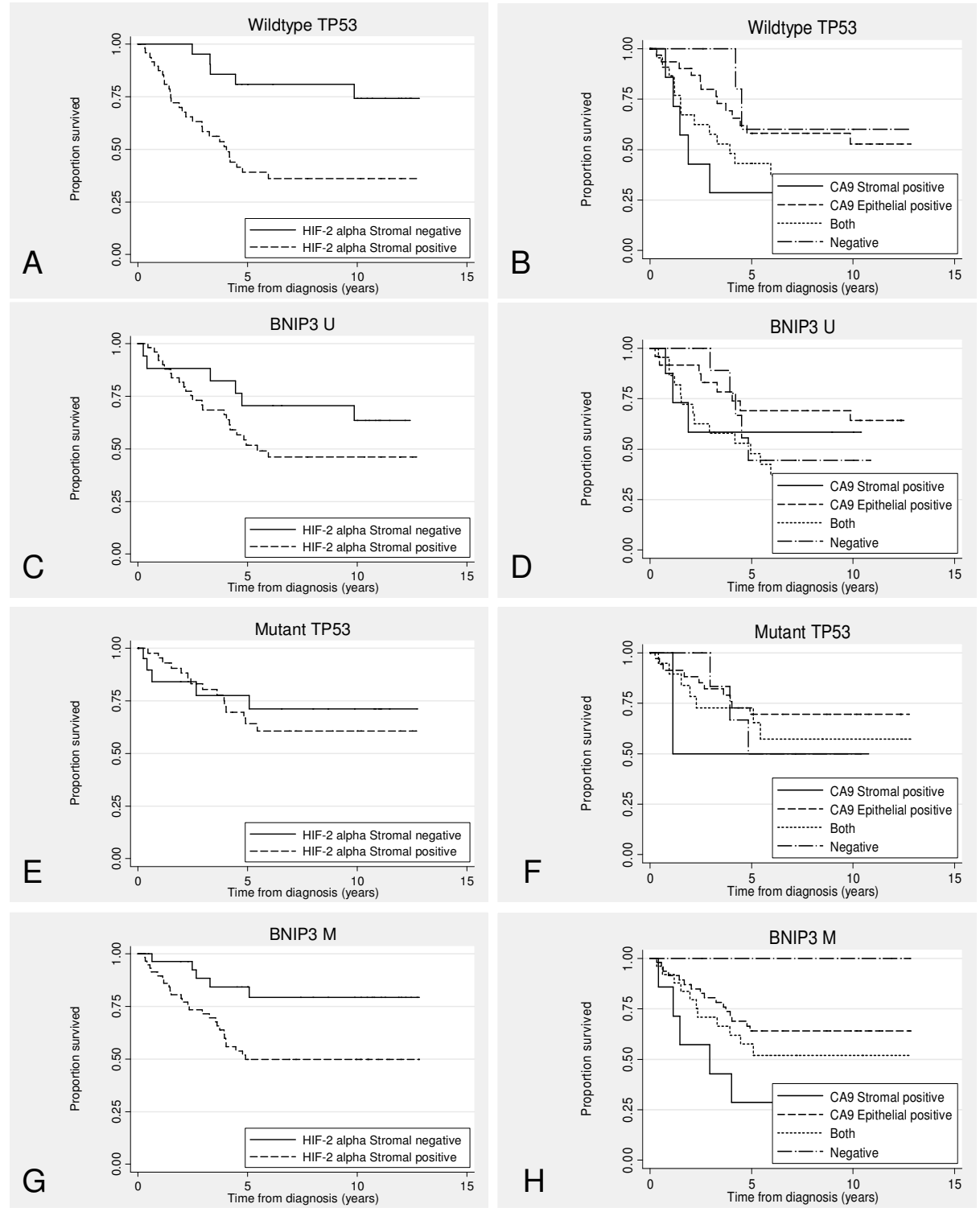

Figure 8.2 Survival curves (A) HIF-2 $\alpha$ expression in wild-type TP53 group, $p=0.001(n=75)$. (B) CA9 expression in wild-type TP53 group, $p=0.0829(n=72)$. (C) HIF-2 $\alpha$ expression in BNIP3 unmethylated group, $\mathrm{p}=0.1712(\mathrm{n}=83$ ). (D) CA9 expression in BNIP3 unmethylated group, $\mathrm{p}=0.0725(\mathrm{n}=79)$. (E) HIF-2 $\alpha$ expression in mutant TP53 group, $\mathrm{p}=0.9312(\mathrm{n}=69)$. (F) CA9 expression in mutant TP53 group $\mathrm{p}=0.8456$ $(n=71)$. (G) HIF-2 $\alpha$ expression in BNIP3 methylated group $p=0.006(n=98)$. (H) CA9 expression in BNIP3 methylated group $\mathrm{p}=0.0495$ ( $\mathrm{n}=95)$. U, Unmethylated; $M$, Methylated. 
154 Chapter 8

Table 8.3 Correlations between BNIP3 methylation and TP53 alteration vs. M30 expression

\begin{tabular}{llllll}
\hline & $\begin{array}{l}\text { M30 } \\
\text { LOW }\end{array}$ & & $\begin{array}{l}\text { M30 } \\
\text { HIGH }\end{array}$ & \\
\hline BNIP3 & No & $\%$ & No & $\%$ & p-value \\
$\quad$ Unmethylated & 50 & $(68)$ & 23 & $(32)$ & .004 \\
$\quad \begin{array}{l}\text { Methylated } \\
\text { TP53 }\end{array}$ & 42 & $(46)$ & 49 & $(54)$ & \\
$\quad$ Wild-type & 39 & $(56)$ & 31 & $(44)$ & .703 \\
$\quad$ Mutation & 36 & $(59)$ & 25 & $(41)$ & \\
\hline
\end{tabular}

\section{Discussion}

In a previous study on the expression of hypoxia related markers (HIF1 $\alpha$, HIF2a, CA9 and GLUT1) in colorectal adenocarcinomas, we found that in all tumors at least one of these proteins is immunohistochemically expressed. This indicates that hypoxia is an all but ubiquitous phenomenon in colorectal tumors. However, only expression of HIF2 $\alpha$ and CA9 in the tumor associated stroma was correlated with a poorer prognosis, suggesting that tumors with this particular phenotype follow a more aggressive course ${ }^{21}$. These results are in contrast to some other studies in colon and rectum tumors, that can be summarized by the observation that HIF1a and GLUT1 expression in rectal cancer cells is of prognostic significance ${ }^{25,38-40}$. Our finding was relatively new, in the sense that others have mainly reported the relation between tumor prognosis and expression of hypoxia markers in cancer cells, without paying attention to stromal expression. The results in our study did not differ between tumors of the colon or rectum.

So far, the biological basis of stromal expression of hypoxia related markers in CRC is unclear. It could either be a serendipitous finding, or a genuine indication of altered epithelial-mesenchymal interactions within a subset of tumors. In these tumors, hypoxia driven metabolic and cell biological changes could hypothetically alter the tumor stroma towards an environment that can facilitate cancer progression, along multiple routes, among which an enhanced proliferation or survival of tumor epithelial cells ${ }^{41}$. The tumor stroma under hypoxic conditions could select for the propagation of certain subclones of cancer cells that are optimally endowed for tumor progression. A recent study in breast cancer, on how changes in stromal gene expression affect epithelial tumor progression, showed that gene expression profiling of microdissected tumor stroma resulted in a set of stromal genes, that could predict clinical outcome. This set notably included enhanced stromal expression of hypoxiaassociated genes ${ }^{42}$. 
The current study attempts to pinpoint specific genetic and epigenetic features of colorectal cancer cells, that are both associated with the observed more aggressive presentation of CRCs expressing HIF2 $\alpha$ and CA9 in their surrounding stroma, and impact on one of the hallmarks of cancer, namely regulation of apoptosis under hypoxic conditions.

Tumor hypoxia results in the induction of pro-death signals, mediated partly by TP53 and $B N I P 3^{43}$. Therefore it could be envisaged that hypoxia provides a selective environment for outgrowth of cells in which these genes have become mutated or silenced. Loss of pro-death genes such as TP53 and BNIP3 may result in increased hypoxia tolerance and cross resistance to other death inducing stimuli associated with metabolic stress or treatment. Immunohistochemical staining showed that TP53 and BNIP3 expression are confined to the epithelial cell compartment in $\mathrm{CRC}$, and are not present in surrounding mesenchymal cells, making these two genes interesting candidates to study the hypothesis of cancer cell selection under hypoxia driven modification of tumor-stroma interactions.

Intriguingly, our results indicate that tumors expressing HIF-2 $\alpha$ or CA9 in their stroma have a poorer prognosis in TP53 wild-type tumors compared to mutant tumors. It is unclear how wild-type TP53 might benefit this tumor subgroup, but several possibilities exist. Firstly, p53 is involved in a metabolic switch to glycolysis when oxidative phosphorylation is impaired during hypoxia ${ }^{44}$. Also, other means of adaptation to metabolic stress, such as an increased fatty acid $\beta$ oxidation, have been shown to be present in tumor cells with intact p53. With respect to this, increased apoptosis was reported in p53-deficient HCT-116 colorectal cancer cells as compared to wild-type p53 HCT-116 cells, when challenged by metabolic stress ${ }^{45}$. The second possibility is that wild-type TP53 is not acting directly, but instead simply correlates with defects in another pathway such as the BNIP3 cell death pathway, that substitutes for TP53 loss in a similar fashion during carcinogenesis.

With respect to apoptosis and hypoxia driven tumor progression, we did not find important effects related to the mutational status of TP53. However, apoptotic levels were lower in BNIP3 expressing tumors, compared to tumors with epigenetically silenced BNIP3 ( $p=0.004)$, which is somewhat surprising, given the fact that functional BNIP3 is thought to induce cell death downstream of hypoxia inducible transcription factors. Apparently, things are more complicated. BNIP3 may not be restricted to regulation of apoptosis, but could also regulate other pathways such as autophagy, in which there is a delicate balance between cell survival and cell death ${ }^{46}$. Conceivably, the lower apoptotic activity in tumors with functional BNIP3 might be due to autophagic rescue of the tumor cells. Furthermore, BNIP3 levels appear to modulate cell death not only via apoptosis or autophagy, but also by necrosis. Also, the net effect of BNIP3 is determined by the level of expression: too high BNIP3 
expression will lead to autophagic cell death, while lower levels of BNIP3 expression, as in cells where BNIP3 is silenced, will induce necrosis ${ }^{47}$. In our study we used the immunohistochemical marker M30, which exclusively measures apoptotic cell death, and thus were not able to differentiate between other forms of cell death, such as autophagic death and necrosis ${ }^{48,49}$.

Summarizing the results from the current study, levels of apoptosis do not play an important role in determining the poorer prognosis of hypoxic CRC's, as defined by stromal expression of HIF-2 $\alpha$ and CA9. However, the latter phenotype is correlated with the presence of wild-type TP53 in the tumor cells, and the presence of functional p53 does indeed appear to have an important impact on poorer prognosis. This prognostic effect is not established through regulation of programmed tumor cell death, but may rather be connected to an enhanced capacity for adequate adaptation to metabolic stress. As TP53 mutations occur in a relatively early stage of colorectal carcinogenesis, the potential deleterious effects of hypoxia on colorectal cancer biology may already be programmed in a very early phase of tumor development.

As opposed to TP53, functional or epigenetically silenced BNIP3 did not turn out to be of influence in determining tumor prognosis. Furthermore, preservation of BNIP3 function showed to decrease apoptotic activity, and may thus be involved in enhanced cell survival through autophagic rescue, or could be implicated in alternative cell death pathways, such as autophagic cell death or necrosis, which we were unable to measure in our experimental approach.

The findings in this translational study, on the relation between expression patterns of hypoxia related markers in clinical samples of CRC and the functional status of genetically or epigenetically modified proteins involved in regulation of tumor cell death on the one hand and patient outcome on the other, opens up interesting new avenues for more fundamental studies on the mechanisms underlying tumor hypoxia induced changes in epithelialmesenchymal interactions.

Understanding the mechanisms by which hypoxic tumors can overcome cell death signals and adapt through metabolic changes is critical for our understanding of tumor progression and development of effective therapeutics in $\mathrm{CRC}$ patients with adverse prognostic profiles. 
TP53, BNIP3 and poor outcome in HIF-2 $\alpha$ and CA9 positive CRCs

\section{References}

1. Erler JT, Bennewith KL, Nicolau M, et al: Lysyl oxidase is essential for hypoxia-induced metastasis. Nature 440:1222-6, 2006

2. Keith B, Simon MC: Hypoxia-inducible factors, stem cells, and cancer. Cell 129:465-72, 2007

3. Bacon AL, Harris AL: Hypoxia-inducible factors and hypoxic cell death in tumour physiology. Ann Med 36:530-9, 2004

4. Swietach $\mathrm{P}$, Vaughan-Jones RD, Harris AL: Regulation of tumor $\mathrm{pH}$ and the role of carbonic anhydrase 9. Cancer Metastasis Rev 26:299-310, 2007

5. Graeber TG, Osmanian C, Jacks T, et al: Hypoxia-mediated selection of cells with diminished apoptotic potential in solid tumours. Nature 379:88-91, 1996

6. Pan Y, Oprysko PR, Asham AM, et al: p53 cannot be induced by hypoxia alone but responds to the hypoxic microenvironment. Oncogene 23:4975-83, 2004

7. Toledo F, Wahl GM: Regulating the p53 pathway: in vitro hypotheses, in vivo veritas. Nat Rev Cancer 6:909-23, 2006

8. Schmaltz $\mathrm{C}$, Hardenbergh $\mathrm{PH}$, Wells $\mathrm{A}$, et al: Regulation of proliferation-survival decisions during tumor cell hypoxia. Mol Cell Biol 18:2845-54, 1998

9. Soussi T, Lozano G: p53 mutation heterogeneity in cancer. Biochem Biophys Res Commun 331:834-42, 2005

10. Kato S, Han SY, Liu W, et al: Understanding the function-structure and function-mutation relationships of p53 tumor suppressor protein by high-resolution missense mutation analysis. Proc Natl Acad Sci U S A 100:8424-9, 2003

11. Fei $\mathrm{P}$, Wang $\mathrm{W}, \mathrm{Kim} \mathrm{SH}$, et al: Bnip3L is induced by p53 under hypoxia, and its knockdown promotes tumor growth. Cancer Cell 6:597-609, 2004

12. Lee H, Paik SG: Regulation of BNIP3 in normal and cancer cells. Mol Cells 21:1-6, 2006

13. Vande Velde C, Cizeau J, Dubik D, et al: BNIP3 and genetic control of necrosis-like cell death through the mitochondrial permeability transition pore. Mol Cell Biol 20:5454-68, 2000

14. Ray R, Chen G, Vande Velde $\mathrm{C}$, et al: BNIP3 heterodimerizes with $\mathrm{Bcl}-2 / \mathrm{Bcl}-\mathrm{X}(\mathrm{L})$ and induces cell death independent of a Bcl-2 homology $3(\mathrm{BH} 3)$ domain at both mitochondrial and nonmitochondrial sites. J Biol Chem 275:1439-48, 2000

15. Mellor HR, Harris AL: The role of the hypoxia-inducible BH3-only proteins BNIP3 and BNIP3L in cancer. Cancer Metastasis Rev 26:553-66, 2007

16. Abe T, Toyota M, Suzuki H, et al: Upregulation of BNIP3 by 5-aza-2'-deoxycytidine sensitizes pancreatic cancer cells to hypoxia-mediated cell death. J Gastroenterol 40:504-10, 2005

17. Yan J, Yun H, Yang Y, et al: Upregulation of BNIP3 promotes apoptosis of lung cancer cells that were induced by p53. Biochem Biophys Res Commun 346:501-7, 2006

18. Okami J, Simeone DM, Logsdon CD: Silencing of the hypoxia-inducible cell death protein BNIP3 in pancreatic cancer. Cancer Res 64:5338-46, 2004

19. Bacon AL, Fox S, Turley $\mathrm{H}$, et al: Selective silencing of the hypoxia-inducible factor 1 target gene BNIP3 by histone deacetylation and methylation in colorectal cancer. Oncogene 26:132-41, 2007

20. Murai M, Toyota M, Suzuki $\mathrm{H}$, et al: Aberrant methylation and silencing of the BNIP3 gene in colorectal and gastric cancer. Clin Cancer Res 11:1021-7, 2005

21. Cleven $A H$, van Engeland M, Wouters BG, et al: Stromal expression of hypoxia regulated proteins is an adverse prognostic factor in colorectal carcinomas. Cell Oncol 29:229-40, 2007

22. Wiggers $\mathrm{T}$, Jeekel $\mathrm{J}$, Arends $\mathrm{JW}$, et al: No-touch isolation technique in colon cancer: a controlled prospective trial. Br J Surg 75:409-15, 1988

23. Herman JG, Graff JR, Myohanen S, et al: Methylation-specific PCR: a novel PCR assay for methylation status of CpG islands. Proc Natl Acad Sci U S A 93:9821-6, 1996

24. Derks S, Lentjes MH, Hellebrekers DM, et al: Methylation-specific PCR unraveled. Cell Oncol 26:291-9, 2004

25. Yoshimura H, Dhar DK, Kohno H, et al: Prognostic impact of hypoxia-inducible factors 1alpha and 2alpha in colorectal cancer patients: correlation with tumor angiogenesis and cyclooxygenase-2 expression. Clin Cancer Res 10:8554-60, 2004 
26. de Bruin EC, van de Velde CJ, van de Pas S, et al: Prognostic value of apoptosis in rectal cancer patients of the dutch total mesorectal excision trial: radiotherapy is redundant in intrinsically high-apoptotic tumors. Clin Cancer Res 12:6432-6, 2006

27. Marijnen CA, Nagtegaal ID, Mulder-Stapel AA, et al: High intrinsic apoptosis, but not radiation-induced apoptosis, predicts better survival in rectal carcinoma patients. Int J Radiat Oncol Biol Phys 57:434-43, 2003

28. Borresen-Dale AL, Lothe RA, Meling Gl, et al: TP53 and long-term prognosis in colorectal cancer: mutations in the L3 zinc-binding domain predict poor survival. Clin Cancer Res 4:20310,1998

29. Conlin A, Smith G, Carey FA, et al: The prognostic significance of K-ras, p53, and APC mutations in colorectal carcinoma. Gut 54:1283-6, 2005

30. Cripps KJ, Purdie CA, Carder PJ, et al: A study of stabilisation of p53 protein versus point mutation in colorectal carcinoma. Oncogene 9:2739-43, 1994

31. Garrity MM, Burgart LJ, Mahoney MR, et al: Prognostic value of proliferation, apoptosis, defective DNA mismatch repair, and p53 overexpression in patients with resected Dukes' B2 or C colon cancer: a North Central Cancer Treatment Group Study. J Clin Oncol 22:1572-82, 2004

32. Kressner U, Inganas M, Byding S, et al: Prognostic value of p53 genetic changes in colorectal cancer. J Clin Oncol 17:593-9, 1999

33. Mollevi DG, Serrano T, Ginesta MM, et al: Mutations in TP53 are a prognostic factor in colorectal hepatic metastases undergoing surgical resection. Carcinogenesis 28:1241-6, 2007

34. Petitjean A, Achatz MI, Borresen-Dale AL, et al: TP53 mutations in human cancers: functional selection and impact on cancer prognosis and outcomes. Oncogene 26:2157-65, 2007

35. Pricolo VE, Finkelstein SD, Hansen K, et al: Mutated p53 gene is an independent adverse predictor of survival in colon carcinoma. Arch Surg 132:371-4; discussion 374-5, 1997

36. Tang R, Wang JY, Fan CW, et al: p53 is an independent pre-treatment markers for long-term survival in stage II and III colorectal cancers: an analysis of interaction between genetic markers and fluorouracil-based adjuvant therapy. Cancer Lett 210:101-9, 2004

37. Tortola S, Marcuello E, Gonzalez I, et al: p53 and K-ras gene mutations correlate with tumor aggressiveness but are not of routine prognostic value in colorectal cancer. J Clin Oncol 17:1375-81, 1999

38. Haber RS, Rathan A, Weiser KR, et al: GLUT1 glucose transporter expression in colorectal carcinoma: a marker for poor prognosis. Cancer 83:34-40, 1998

39. Lu XG, Xing CG, Feng YZ, et al: Clinical significance of immunohistochemical expression of hypoxia-inducible factor-1alpha as a prognostic marker in rectal adenocarcinoma. Clin Colorectal Cancer 5:350-3, 2006

40. Theodoropoulos GE, Lazaris AC, Theodoropoulos VE, et al: Hypoxia, angiogenesis and apoptosis markers in locally advanced rectal cancer. Int $\mathrm{J}$ Colorectal Dis 21:248-57, 2006

41. Beppu H, Mwizerwa ON, Beppu Y, et al: Stromal inactivation of BMPRII leads to colorectal epithelial overgrowth and polyp formation. Oncogene 27:1063-70, 2008

42. Finak G, Bertos N, Pepin F, et al: Stromal gene expression predicts clinical outcome in breast cancer. Nat Med 14:518-27, 2008

43. Brahimi-Horn C, Pouyssegur J: The role of the hypoxia-inducible factor in tumor metabolism growth and invasion. Bull Cancer 93:E73-80, 2006

44. Lum JJ, Bui T, Gruber M, et al: The transcription factor HIF-1alpha plays a critical role in the growth factor-dependent regulation of both aerobic and anaerobic glycolysis. Genes Dev 21:1037-49, 2007

45. Buzzai M, Jones RG, Amaravadi RK, et al: Systemic treatment with the antidiabetic drug metformin selectively impairs p53-deficient tumor cell growth. Cancer Res 67:6745-52, 2007

46. Papandreou I, Krishna C, Kaper F, et al: Anoxia is necessary for tumor cell toxicity caused by a low-oxygen environment. Cancer Res 65:3171-8, 2005

47. Tracy K, Dibling BC, Spike BT, et al: BNIP3 is an RB/E2F target gene required for hypoxiainduced autophagy. Mol Cell Biol, 2007 
TP53, BNIP3 and poor outcome in HIF-2 $\alpha$ and CA9 positive CRCs

48. Leers MP, Kolgen W, Bjorklund V, et al: Immunocytochemical detection and mapping of a cytokeratin 18 neo-epitope exposed during early apoptosis. J Pathol 187:567-72, 1999

49. Ueno $\mathrm{T}$, Toi M, Linder S: Detection of epithelial cell death in the body by cytokeratin 18 measurement. Biomed Pharmacother 59 Suppl 2:S359-62, 2005 
Table 8.1S TP53 mutation analysis primers and BNIP3 MSP primer sequences.

\begin{tabular}{|c|c|c|c|}
\hline TP53 & Primer & Sequence & Product size \\
\hline \multirow[t]{3}{*}{5 exon } & Flank sense & 5' CTG TTC ACT TGT GCC CTG AC 3' & 268 bp \\
\hline & Flank antisense & 5' AGC CCT GTC GTC TCT CCA G 3' & \\
\hline & Inside sense & 5' TGC CCT GAC TTT CAA CTC TG 3' & 257 bp \\
\hline \multirow[t]{3}{*}{6 exon } & Flank sense & 5' GGC CTC TGA TTC CTC ACT G 3' & $240 \mathrm{bp}$ \\
\hline & Flank antisense & 5' ATA AGC AGC AGG AGA AAG CC 3' & \\
\hline & Inside antisense & 5’ GAC CCC AGT TGC AAA CCA G 3' & 164 bp \\
\hline \multirow[t]{2}{*}{7 exon } & Flank sense & 5' CAT CTT GGG CCT GTG TTA TC 3' & $261 \mathrm{bp}$ \\
\hline & $\begin{array}{l}\text { Flank antisense } \\
\text { Inside antisense }\end{array}$ & $\begin{array}{l}\text { 5' GAT GTG ATG AGA GGT GGA TGG 3' } \\
\text { 5' GGA AGA AAT CGG TAA GAG GTG 3' }\end{array}$ & $230 \mathrm{bn}$ \\
\hline \multirow[t]{3}{*}{8 exon } & Flank sense & 5' GAT TTC CTT ACT GCC TCT TGC 3' & $231 \mathrm{bp}$ \\
\hline & Flank antisense & 5' CAT AAC TGC ACC CTT GGT CTC 3, & \\
\hline & Inside antisense & 5’ TCC TCC ACC GCT TCT TGT C 3' & 212 bp \\
\hline \multirow[t]{7}{*}{ BNIP3 } & Primer & Sequence & Product size \\
\hline & Flank Sense & 5'TTY GTT TTG TTT TGT GAG TTT TTT-3' & $129 \mathrm{bp}$ \\
\hline & $\begin{array}{l}\text { Flank } \\
\text { antisense }\end{array}$ & 5'-CCR AAC TAC AAA ATA TAC TTC AAC TAC-3' & \\
\hline & $\begin{array}{l}\text { Unmethylated } \\
\text { sense }\end{array}$ & $\begin{array}{l}\text { 5'-GGT TTT GTT TAG TTT GGG AGT G-3' } \\
\text { 5'-CCT CAA CTA CAA ACA ATA AAA AAA CA-3, }\end{array}$ & 74 bp \\
\hline & $\begin{array}{l}\text { Unmethylated } \\
\text { antisense }\end{array}$ & & \\
\hline & $\begin{array}{l}\text { Methylated } \\
\text { sense }\end{array}$ & $\begin{array}{l}\text { 5'-TTT CGT TTA GTT CGG GAG CG-3' } \\
\text { 5'-CAA CTA CGA ACG ATA AAA AAA CG-3' }\end{array}$ & 69 bp \\
\hline & $\begin{array}{l}\text { Methylated } \\
\text { antisense }\end{array}$ & & \\
\hline
\end{tabular}




\section{Chapter}

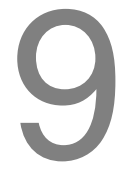

General discussion 
$162 \mid$ Chapter 9 
General discussion $\mid 163$

\section{General discussion and future perspectives}

Accurate staging of colorectal cancer (CRC) is of crucial importance for optimal management of the disease. The currently used classification system, the American Joint Committee on Cancer (AJCC) TNM classification system is based on three variables: the extent of penetration of tumor into the intestinal wall ( $\mathrm{T}$ stage), nodal status ( $\mathrm{N}$ stage) and the presence of distant metastasis (M stage). Although easy to implement, the survival for patients with the same TNM stage varies, which is caused by other factors that influence the prognosis. Therefore, the TNM classification is regularly being revised in order to improve the prediction of outcome. For example, a large study $(n=128,000)$ including readily available patient characteristics such as age, sex, tumor differentiation/grade, and number of regional lymph nodes evaluated, better predicts survival than the currently used seventh edition of the AJCC system ${ }^{1}$. This study clearly indicates that adding additional factors influencing prognosis can be helpful in refining the staging system for CRC.

Since the behavior of the tumor is determined by the underlying biology, inclusion of biological aspects of CRC carcinogenesis into the currently used classification system would be the next step. The last two decades it has become clear that $\mathrm{CRC}$ is a heterogeneous disease consisting of multiple molecular subtypes differentially influencing prognosis and response to therapy. The molecular subgroups most often used are MSI, CIN and CIMP, however only for MSI CRCs a consistent and accepted positive association with prognosis has been reported ${ }^{2}$. Moreover, in contrast to assessing KRAS mutations to predict response to anti-EGFR therapy ${ }^{3-5}$ and establishing the cause of $\mathrm{MLH} 1$ loss $^{6}$ (biallelic $M L H 1$ promoter $\mathrm{CpG}$ island methylation in sporadic $\mathrm{CRC}$ or mutations in mismatch repair genes in patients with Lynch syndrome) to determine disease management, this information is currently not being used routinely in the clinic.

In addition to the molecular classification mentioned above, a revolution in the technologies to study the molecular alterations underlying the initiation and development of cancer, such as micro-array- and next generation sequencing approaches enabled a detailed exploration of the genetic and epigenetic basis of CRC. Thus, although the identification of subgroups and key molecular alterations in CRC are rapidly being unraveled, the majority of these alterations have not been confirmed and validated as biomarkers in large validation studies. Multiple reasons account for this. First, independent validation studies confirming the initial observation are often lacking. Second, data analyses are predominantly performed on a mixed group of CRCs, not taking into account specific subgroups. Third, the effect of treatment is not always accounted for. The aim of this thesis was to assess well characterized genetic and epigenetic alterations in a retrospective study, consisting of patients with CRC who were 
entered in two multi-center prospective clinical trials between 1979 and 1981 in the Netherlands and were treated by surgery alone. We studied these alterations in relation with morphological features, hypoxia and prognosis.

First we analysed a set of well characterized molecular parameters associated with CRC, e.g. MSI, CIMP, APC-, KRAS-, TP53-, and BRAF mutations in a series of $160 \mathrm{CRC}$ cases. This study was based on a literature analysis by Jass et al. ${ }^{7}$ in 2007 , concluding that five distinct molecular subgroups with differential clinicopathological and morphologic features can be identified in CRC. We tested this hypothesis by using computer assisted unsupervised hierarchical clustering of the markers described above. Interestingly, hierarchical clustering of colorectal adenocarcinomas showed that CIMP status is the principal classifier, and that both CIMP+ and CIMP- tumors are further classified on the basis of TP53 mutational status. Although it has been more than a decade since CIMP was first identified in CRC, controversy still exists on whether this subgroup of CRCs is an etiologically and clinically distinct group within the disease and to date, the cause of CIMP remains unknown ${ }^{8}$. Even more complexity is introduced by the fact that no gold standard with respect to gene panels, marker thresholds or techniques for detection of the altered DNA methylation used to define this phenotype exists ${ }^{8}$. Taking into account these considerations, it is surprising to observe that CIMP was identified as the principal classifier for CRC in this study and although these data need to be replicated, this observation underscores the significance and illustrates the need to establish the group of CRCs characterized by CIMP. Although the subgroups generated by the clustering algorithms did not show strong associations with clinicopathological parameters and therefore could not improve traditional staging with respect to identifying patients with a poorer survival in our first study, we did find an association with CIMP and prognosis in the validation series analysed in Chapter 4 . We also observed that, in line with other publications ${ }^{7,9,10}, \mathrm{CIMP}+\mathrm{CRC}$ appear to comprise two subsets, one localized in the right-sided colon, with $\mathrm{MSI}$ and mucinous differentiation, the other occurring in the rectum, being $\mathrm{MSS}^{7,9,10}$. This could reflect important differences in the underlying molecular biology of proximal and distal CRCs. For example, is was recently reported that anatomic location is a more significant predictor of progression than the histological type of CRC precursors like hyperplastic polyps, sessile serrated polyp or traditional adenoma ${ }^{11}$. One explanation for a difference in biology could be a difference in the availability of dietary factors important in promoter $\mathrm{CpG}$ island methylation such as folic acid, methionine and related vitamins B2, B6 and B12. Epidemiological evidence suggests that these nutrients are associated with risk of colorectal cancer and CIMP status ${ }^{12-15}$. Notably, methionine has been associated with a decreased risk of proximal colon cancer, whilst among women it was inversely associated with rectal cancer. Conversely, a strong positive association between vitamin 
B6 and rectal cancer risk in women has been reported ${ }^{14}$. These results underscore that nutrients involved in promoter CpG island methylation are to some extent related to associated phenotypes such as CIMP and tumor location in the large intestine.

Since we observed a strong effect of CIMP on the clustering in this series, we further investigated whether CIMP and 19 other genes reported to be functionally methylated and involved in a diversity of cancer related pathways in CRC, were associated with prognosis. Again, keeping in mind that prognosis is affected by biology of subgroups of cancer and treatment, we specifically analyzed data taking into account defined subgroups (based on MSI and $B R A F$-mutation status, other genetic alterations had no significant effect on prognosis) of CRCs in the population only treated with surgery.

Although for CIMP no clear biological cause or definition exists and numerous methods and definitions are used in CRC to quantify $\mathrm{CIMP}^{8,16}$ we chose to use the Weisenberger panel ${ }^{17}$ for studying associations with clinicopathological variables. When analyzing all 19 (or 24 if including CIMP) genes, only APC and CHFR promoter CpG island methylation were associated with a worse prognosis in stage II MSS, BRAF wild-type CRCs. For stage III MSS BRAF wildtype CRCs, associations with a poorer prognosis were found for promoter $\mathrm{CpG}$ island methylation of RASSF1A, THBS1, CACNA1G. When analyzing a second, independent series of CRCs, we could only confirm the prognostic effect of CHFR methylation in stage II, MSS, BRAF wildtype CRCs. This last observation illustrates the importance of validating findings in independent series. Promoter $\mathrm{CpG}$ island methylation of CHFR is present in approximately $43 \%$ of CRCs and is not a prognostic marker when assessed in a general population of CRCs consisting of all stages and all subgroups. Analyzing this gene in a specific subgroup of CRCs, namely the stage II MSS BRAF wildtype CRCs reveals the potential of this marker. Since we only observed this effect in two series of CRCs, we searched the literature for additional evidence for CHFR being a clinically relevant gene (Chapter 5). Interestingly, we found multiple lines of evidence for CHFR not only having prognostic significance but also predictive significance. CHFR is a E3 ubiquitin-protein ligase and functions as a mitotic stress checkpoint protein that delays mitotic entry in response to microtubule damage ${ }^{18}$. Although taxanes are not implemented in CRC treatment because they failed to demonstrate a significant clinical benefit in Phase II trials ${ }^{19,20}$, a specific subgroup of CRCs with CHFR promoter methylation might benefit from taxanes. Therefore additional studies comparing the outcome of stage II MSS BRAF wildtype CHFR methylated CRCs versus unmethylated stage II MSS BRAF wildtype CRCs and the effect of taxane treatment in CHFR methylated versus unmethylated CRCs is warranted. Although we found multiple lines of evidence, some inconsistent findings have 
been reported as well. This clearly shows the need for large, well designed trials to test the clinical relevance of CHFR methylation in CRC.

The first chapters of this thesis in detail address the genetic and epigenetic changes that occur during colorectal carcinogenesis, and their role in classification of the disease, notably the importance of promoter CpG island methylation in subcategorizing large intestinal tumors, which can further be characterized by certain genetic alterations, of which TP53 mutations and microsatellite stability status appear to be the most relevant. These studies also show that CIMP is a more complex entity than was previously anticipated, not only with respect to definition and mode of detection, but CIMP also represents a heterogeneous category of tumors, from which an unequivocal single prognostic or predictive role for the whole group cannot be derived. Rather, it turns out that methylation of invididual genes may play specific roles in the process of tumorigenesis and tumor progression, as exemplified by CHFR. In the second part of this thesis we therefore continued our search for additional individual candidate methylated genes, which not only could be applied in classification, prognostication and therapy of $\mathrm{CRC}$, but might play a role in tumor biology as well. These studies depart from the notion that promoter $\mathrm{CpG}$ island methylation is an event that occurs early in neoplasia, and can even be demonstrated in pre-neoplasia, which in the colorectum is exemplified by gene methylation in adenomas ${ }^{21,22}$. As promoter $\mathrm{CpG}$ island methylation subsequently remains present during later evolutionary stages of malignancy, we presumed that this type of epigenetic change not just accompanies tumor progression, but might exert it's influence on tumor progression as well. Apart from accumulation of genetic and epigenetic changes, tumor progression in a biological sense involves the concerted interaction of several important pathways, which have been defined as the so-called "hallmarks of cancer" ${ }^{\text {"23 }}$. In chapter 7, we therefore studied promoter CpG island methylation in a selection of multiple genes which individually can be attributed to every one of these tumor progression pathways. Furthermore, as tumors become increasingly dependent on oxygen and nutrients during progression, and upon outgrowing their supply become increasingly hypoxic, we correlated the methylation status of the selected genes with the occurrence of hypoxia in colorectal adenocarcinomas, defined by immunohistochemical expression of hypoxia related markers. We specifically chose to correlate epigenetic changes with such a complex phenomenon as hypoxia, because the latter has a major impact on cancer biology, not only underlying genetic, biochemical and metabolic changes in carcinoma cells, but also in a more integrative way, coordinating cellular alterations and responses in the tumor micro-environment, and influencing the interaction between tumor cells and stroma. With respect to this, in our studies, we did not restrict our observations to the tumor epithelial 
compartment, but also specifically addressed stromal changes. Finally, tumor hypoxia is important from a clinical perspective, driving tumor progression towards a more aggressive phenotype, with relative resistance to chemotherapy and radiotherapy.

In the studies described in chapters 6 and 7 we found an important role for the tumor stroma (as opposed to tumor epithelial cells), with respect to both expression of hypoxia related markers, the relation of this expression pattern with a more aggressive tumor behavior, and the correlation with promoter $\mathrm{CpG}$ island methylation of selected genes involved in tumor progression pathways. This points to a potentially important role for the tumor micro-environment during tumorigenesis. It has already been well established that tumor development and -biology cannot be explained exclusively by studying tumor cells, but are modified to a considerable extent through the interaction with surrounding stromal cells. Work by other groups in recent years supports the somewhat underestimated role of the tumor stroma in determining the biology of a malignant tumor. With respect to this, in general, Pietras and Ostman review the role of tumor stroma and specific mesenchymal cell types in governing processes involved in the hallmarks of cancer $^{24}$, while others describe the role of hypoxia in relation to these hallmarks ${ }^{25}$. Our results add to these, by linking tumor hypoxia, promoter $\mathrm{CpG}$ island methylation of selected genes, and the tumor stroma. Of course our studies are correlative in nature and do not allow any definitive conclusions on the underlying mechanisms. There are however more detailed studies reported in the literature, underpinning the relevance of stromal changes for tumor development and biology. Recently it was revealed that stromal cell signals could lead to silencing of tumor suppressor genes in tumor cells by promoter $\mathrm{CpG}$ island methylation ${ }^{26}$. It was shown that the epigenome of tumor cells is highly plastic and easily modified by the tumor microenvironment ${ }^{26-32}$. Similarly as within tumor cells, stromal cells also harbour unique aberrant epigenomes, relevant to clinical outcome and treatment choices ${ }^{33}$. Finak et al. ${ }^{34}$ supply evidence for the supplementary role of tumor stroma in governing tumor biology. In their study on breast cancer, they addressed stromal gene expression through a microdissection approach, separating tumor stroma from epithelium, and found an interesting set of specific stromally expressed genes, which have an impact on several tumor biological mechanisms, and which they combined into a new stroma derived prognostic predictor set. Interestingly, the stromal genes that were upregulated in their study included genes involved in immune responses, angiogenic and hypoxic responses. Also other studies report genomic alterations in stroma relevant to tumor development ${ }^{33}$. An interesting mechanistic and theoretical approach was presented by Lisanti et al. ${ }^{35}$, describing the role of hypoxia, oxidative stress and autophagy/mitophagy in the tumor microenvironment. In their so-called "Autophagic Tumor Stroma Model of 
Cancer", the theoretical basis is that during tumor growth cancer cells induce oxidative stress in surrounding stroma, which leads to production of reactive oxygen species in the stromal compartment. These subsequently give rise to upregulation of defense mechanisms in the epithelial compartment, which not only protects against oxidative stress, but also serves to prevent tumor cell apoptosis. Moreover, stromal production of reactive oxygen species can induce mutations and genomic instability in the tumor epithelium. Also, stromal autophagy and mitophagy increase, and this process of degradation gives rise to enhanced production of metabolites such as ketones, pyruvate and lactate in the tumor microenvironment, which in turn serves to fuel the tumor cells, enabling tumor growth. Naturally, intricate signaling and sophisticated crosstalk between stromal cells and tumor epithelial cells take place during the course of tumor progression. In this respect, HIF transcription factors and their downstream effects play a central part, but it seems plausible and appealing that methylation and silencing of certain key regulatory genes connected to various tumor progression pathways are involved as well.

As was presented and discussed in Chapter 7, we found an interesting correlation between tumor hypoxia, represented by stromal expression of hypoxia markers, and methylation of certain genes involved in essential tumor progression pathways. Whether the process of methylation is a cause or rather a consequence of hypoxia is still a matter of debate. We were interested in another aspect of gene methylation, namely the ability to protect tumor cells from the deleterious effects of tumor hypoxia. Hypoxia in theory induces cell death, and thus can render selective pressure on a tumor cell population, in which only the most resilient, apoptosis insensitive tumor cells would survive and continu to populate the tumor with the most aggressive cell fractions. We therefore chose to address one of the hallmarks of cancer, the evasion of apoptosis, in an attempt to clarify this issue. For this purpose, in chapter 8 , the correlations of BNIP3 methylation and TP53 mutational status with levels of apoptosis and tumor behaviour were investigated. The idea being that silencing of pro-apoptotic BNIP3 in tumor cells could protect them from the occurrence of programmed cell death, and along similar lines, that mutations in TP53 would result in defective apoptotic machinery, and thus that both events would lead to lower apoptotic activity in a tumor. The hypothesis was that tumors with BNIP3 methylation and/or TP53 mutations would have the lowest apoptotic activity, the most accelerated growth, leading to increased hypoxia, and show the most aggressive behaviour, as manifested by the poorest prognosis. However, it turned out that wild-type TP53 did not have an effect on apoptosis levels but was associated with the more aggressive tumors with stromal hypoxia, whereas conversely, tumors with unmethylated and thus preserved BNIP3 had lower apoptotic levels, and this on the other hand did not have an impact on tumor prognosis. It was reasoned that functional p53 could act in other ways to 
protect cells from the selective pressure exerted by hypoxic stress, such as metabolic adaptation of tumor cells to hypoxia. As BNIP3 is also known to be involved in autophagy, perhaps alternative causes of cell death come into play in tumors unmethylated for this gene, which could still account for the lack of correlation with tumor aggressiveness and poorer prognosis. These results in Chapter 8 once again show that there is a considerable gap between theoretical models and hypotheses, the data derived from descriptive studies using patient materials, clinical data and statistical analysis on the one hand and the ability to explain important tumor biological processes on the other. Nevertheless, the data presented in the current thesis will hopefully form a theoretical basis for further mechanistic studies to clarify the precise relations between epigenetic changes, tumor hypoxia and the role of tumor stroma during the process of tumor progression. 


\section{References}

1. Weiser MR, Gonen M, Chou JF, et al: Predicting survival after curative colectomy for cancer: individualizing colon cancer staging. J Clin Oncol 29:4796-802, 2011

2. Ward RL, Cheong K, Ku SL, et al: Adverse prognostic effect of methylation in colorectal cancer is reversed by microsatellite instability. J Clin Oncol 21:3729-36, 2003

3. Hutchins G, Southward K, Handley $\mathrm{K}$, et al: Value of mismatch repair, KRAS, and BRAF mutations in predicting recurrence and benefits from chemotherapy in colorectal cancer. $J$ Clin Oncol 29:1261-70, 2011

4. Pallis $\mathrm{A}$, Briasoulis $\mathrm{E}$, Linardou $\mathrm{H}$, et al: Mechanisms of resistance to epidermal growth factor receptor tyrosine kinase inhibitors in patients with advanced non-small-cell lung cancer: clinical and molecular considerations. Curr Med Chem 18:1613-28, 2011

5. Pallis AG, Karampeazis A, Vamvakas L, et al: Efficacy and treatment tolerance in older patients with NSCLC: a meta-analysis of five phase III randomized trials conducted by the Hellenic Oncology Research Group. Ann Oncol, 2011

6. Kane MF, Loda M, Gaida GM, et al: Methylation of the hMLH1 promoter correlates with lack of expression of hMLH1 in sporadic colon tumors and mismatch repair-defective human tumor cell lines. Cancer Res 57:808-11, 1997

7. Jass JR: Classification of colorectal cancer based on correlation of clinical, morphological and molecular features. Histopathology 50:113-30, 2007

8. Hughes LA, Khalid-de Bakker CA, Smits KM, et al: The CpG island methylator phenotype in colorectal cancer: Progress and problems. Biochim Biophys Acta 1825:77-85, 2011

9. Issa JP, Shen L, Toyota M: CIMP, at last. Gastroenterology 129:1121-4, 2005

10. Sanchez JA, Krumroy L, Plummer S, et al: Genetic and epigenetic classifications define clinical phenotypes and determine patient outcomes in colorectal cancer. Br J Surg 96:1196204, 2009

11. Kim KM, Lee AJ, Ha A, et al: Molecular features of colorectal hyperplastic polyps and sessile serrated adenoma/polyp from Korea. American Journal of Surgical Pathology 35:1274-86, 2011

12. Schernhammer ES, Giovannucci E, Baba Y, et al: B vitamins, methionine and alcohol intake and risk of colon cancer in relation to BRAF mutation and $\mathrm{CpG}$ island methylator phenotype (CIMP). PLoS One 6:e21102, 2011

13. de Vogel S, Bongaerts BW, Wouters KA, et al: Associations of dietary methyl donor intake with MLH1 promoter hypermethylation and related molecular phenotypes in sporadic colorectal cancer. Carcinogenesis 29:1765-73, 2008

14. de Vogel S, Dindore V, van Engeland $M$, et al: Dietary folate, methionine, riboflavin, and vitamin B-6 and risk of sporadic colorectal cancer. J Nutr 138:2372-8, 2008

15. van Engeland M, Weijenberg MP, Roemen GM, et al: Effects of dietary folate and alcohol intake on promoter methylation in sporadic colorectal cancer: the Netherlands cohort study on diet and cancer. Cancer Res 63:3133-7, 2003

16. Hughes LA, Simons CC, van den Brandt PA, et al: Body size, physical activity and risk of colorectal cancer with or without the CpG island methylator phenotype (CIMP). PLoS One 6:e18571, 2011

17. Weisenberger DJ, Siegmund KD, Campan $M$, et al: $C p G$ island methylator phenotype underlies sporadic microsatellite instability and is tightly associated with BRAF mutation in colorectal cancer. Nat Genet 38:787-93, 2006

18. Kim JS, Park YY, Park SY, et al: The Auto-ubiquitylation of E3 Ubiquitin-protein Ligase Chfr at G2 Phase Is Required for Accumulation of Polo-like Kinase 1 and Mitotic Entry in Mammalian Cells. J Biol Chem 286:30615-23, 2011

19. Swanton C, Tomlinson I, Downward J: Chromosomal instability, colorectal cancer and taxane resistance. Cell Cycle 5:818-23, 2006

20. Smits K, Cleven AH, Weijenberg MP, et al: Pharmacoepigenomics in colorectal cancer: a step forward in predicting prognosis and treatment response. Pharmocogenomics 9:1903-16, 2008 
General discussion $\mid 171$

21. Derks S, Postma C, Carvalho B, et al: Integrated analysis of chromosomal, microsatellite and epigenetic instability in colorectal cancer identifies specific associations between promoter methylation of pivotal tumour suppressor and DNA repair genes and specific chromosomal alterations. Carcinogenesis 29:434-9, 2008

22. Derks S, Postma C, Moerkerk PT, et al: Promoter methylation precedes chromosomal alterations in colorectal cancer development. Cell Oncol 28:247-57, 2006

23. Hanahan D, Weinberg RA: The hallmarks of cancer. Cell 100:57-70, 2000

24. Pietras K, Ostman A: Hallmarks of cancer: interactions with the tumor stroma. Exp Cell Res 316:1324-31, 2010

25. Ruan K, Song G, Ouyang G: Role of hypoxia in the hallmarks of human cancer. J Cell Biochem 107:1053-62, 2009

26. Lin $\mathrm{HJ}$, Zuo T, Lin $\mathrm{CH}$, et al: Breast cancer-associated fibroblasts confer AKT1-mediated epigenetic silencing of Cystatin M in epithelial cells. Cancer Res 68:10257-66, 2008

27. Chen D, Reierstad S, Lu M, et al: Regulation of breast cancer-associated aromatase promoters. Cancer Lett 273:15-27, 2009

28. Chiu CF, Wang HC, Wang $\mathrm{CH}$, et al: A new single nucleotide polymorphism in XRCC4 gene is associated with breast cancer susceptibility in Taiwanese patients. Anticancer Res 28:26770,2008

29. Fang F, Flegler AJ, Du P, et al: Expression of cyclophilin B is associated with malignant progression and regulation of genes implicated in the pathogenesis of breast cancer. $\mathrm{Am} \mathrm{J}$ Pathol 174:297-308, 2009

30. Gorringe KL, Choong DY, Visvader JE, et al: BARD1 variants are not associated with breast cancer risk in Australian familial breast cancer. Breast Cancer Res Treat 111:505-9, 2008

31. Ueki T, Nishidate T, Park JH, et al: Involvement of elevated expression of multiple cell-cycle regulator, DTL/RAMP (denticleless/RA-regulated nuclear matrix associated protein), in the growth of breast cancer cells. Oncogene 27:5672-83, 2008

32. Zhao JJ, Lin J, Yang $\mathrm{H}$, et al: MicroRNA-221/222 negatively regulates estrogen receptor alpha and is associated with tamoxifen resistance in breast cancer. J Biol Chem 283:3107986, 2008

33. Eng C, Leone G, Orloff MS, et al: Genomic alterations in tumor stroma. Cancer Res 69:675964, 2009

34. Finak G, Bertos N, Pepin F, et al: Stromal gene expression predicts clinical outcome in breast cancer. Nat Med 14:518-27, 2008

35. Lisanti MP, Martinez-Outschoorn UE, Chiavarina B, et al: Understanding the "lethal" drivers of tumor-stroma co-evolution: emerging role(s) for hypoxia, oxidative stress and autophagy/mitophagy in the tumor micro-environment. Cancer Biol Ther 10:537-42, 2010 
172 
Summary 


\section{Summary}

Colorectal cancer (CRC) is one of the most common cancer types worldwide and approximately $50 \%$ of patients with CRC die due to the disease. The histology based Tumor Node Metastasis (TNM) system is currently the best tool to provide information on prognosis and to determine treatment protocols in CRC. Although this anatomical based staging system predicts the survival accurately, variation in the course of the disease and response to treatment among individuals within the same stage exists. Therefore the identification of new prognostic and predictive markers for $\mathrm{CRC}$ to determine prognosis and to identify patients that need adjuvant therapy respectively is urgently needed. Understanding the underlying biology of CRC will provide novel molecular markers to accurately predict prognosis and improve treatment options besides the traditional histological staging system.

The aim of this thesis was to study genetic and epigenetic alterations in relation to morphological features and hypoxia in CRC, providing insight in the underlying biology of CRC and possibly yielding relevant novel prognostic information.

In chapter 1 we introduce the (epi)-genetic alterations that underlie the process of cancer development and discuss the molecular phenotypes of CRC: chromosomal instability (CIN), microsatellite instability (MSI) and the CpG island methylator phenotype (CIMP). Furthermore, the importance of hypoxia within solid tumors like CRCs is pinpointed, by explaining the cellular responses during hypoxia, with special emphasis on the tumor associated stroma.

In chapter 2, we review the current knowledge on the epidemiology, diagnosis, treatment and the prognostic and predictive value of (epi)genetic markers in CRC.

In the first experimental study (Chapter 3) we classified CRCs by computer assisted unsupervised hierarchical clustering, based upon well described markers in the genesis of CRC including: CIMP, MSI, APC-, KRAS-, TP53-, and BRAF mutation status, in a series of 160 CRCs. Subsequently, we investigated in which way this classification was related to patient data, including clinicopathological parameters, morphological features (differentiation grade, mucinous differentiation, dirty necrosis, circumscribed tumor growth, tumor budding and lymphocyte infiltration) and biological behavior in terms of patient prognosis.

These analyses revealed that for molecular clustering of colorectal adenocarcinomas, CIMP status was the principal classifier, and that both 
CIMP+ and CIMP- tumors were further classified on the basis of TP53 mutational status. Hierarchical clustering was only modestly related to morphology and outcome, which appeared to be dependent on additional factors such as microsatellite stability and localization in the intestine. Although molecular clustering provided insight in the underlying biology of CRC, it did not improve traditional staging with respect to prognostic value.

Data on the prognostic significance of promoter $\mathrm{CpG}$ island methylation in $\mathrm{CRC}$ are conflicting, possibly due to associations between methylation and other factors affecting survival such as genetic alterations and use of adjuvant therapy. Therefore in chapter 4 we examined the prognostic impact of promoter methylation of 19 tumor suppressor- and DNA repair genes and CIMP, as defined by Weisenberger et al., in CRC patients treated with surgery alone in the context of $\mathrm{MSI}, B R A F-$ and KRAS mutations. Our study failed to demonstrate a consistent prognostic effect of CIMP, however we identified promoter CpG island methylation of CHFR as a prognostic biomarker in stage II, MSS, BRAF wild-type CRCs in two independent populations of CRCs.

Chapter 5 reviews the evidence for CHFR as a prognostic and predictive biomarker in a diversity of solid malignancies including CRC.

In CRC, the importance of hypoxia has been demonstrated by clinical studies in which hypoxia predicts for worse outcome and resistance to chemotherapy and radiotherapy. Therefore, in chapter 6 we studied the role of protein

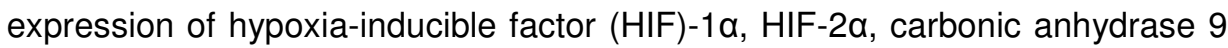
(CA9) and glucose transporter 1 (GLUT1) in patients with colorectal adenocarcinomas. Expression of these hypoxia markers was detected in the epithelial compartment of the tumor cells as well as in tumor associated stromal cells. Although epithelial tumor cells frequently showed expression of one or more of the investigated hypoxia markers, no correlation among these markers or with clinical response was found. In contrast to this, within the tumor stroma, expression of HIF-2 $\alpha$ and CA9 were both associated with a significantly reduced overall survival. In the Cox proportional hazard model, stromal HIF-2a expression was an independent prognostic factor for survival. Our study suggested that tumor hypoxia may influence tumor associated stromal cells in a way that ultimately contributes to patient prognosis.

Since epigenetic silencing might play a role in the cellular responses to hypoxia, in chapter 7 we investigated whether hypoxia in CRC is associated with promoter $\mathrm{CpG}$ island methylation of genes involved in important tumor biological processes such as: MGMT, CHFR, HLTF, MLH1, RASSF1A (genomic instability), p14ARF, p16INK4A (limitless replication potential), 
ADAM23, TFPI2 (tissue invasion and metastasis), APC (insensitivity to antigrowth signals), SOCS1 (self-sufficiency in growth signals), THBS1 (sustained angiogenesis), and BNIP3, NEURL, RUNX3, RAB32 (evasion of apoptosis). This study showed that stromal expression of hypoxia regulated proteins HIF$2 \alpha$ and CA9 was associated with promoter $\mathrm{CpG}$ island methylation of genes involved in important tumor biological processes. Tumors with hypoxic features did not show increased overall $\mathrm{CpG}$ island promoter methylation, but rather harboured specific methylated cancer biology related genes, which could contribute to the more aggressive behaviour of these tumors.

Finally in chapter 8 we studied whether tumor cell death, regulated by a hypoxic stromal microenvironment could be of importance in the observed poorer prognosis in CRC with stromal expression of HIF-2 $\alpha$ and CA9. Therefore, we correlated apoptosis, TP53 mutational status and BNIP3 promoter hypermethylation (both genes involved in regulation of apoptosis) of CRC cells with HIF-2 $\alpha$ and CA9 related poorer outcome. Our study showed that the effect of HIF-2 $\alpha$ as an indicator of a poorer prognoses was dependent on wild-type TP53 and not methylation status of BNIP3. It appeared that wildtype TP53 in CRC cells favors progression of tumors expressing markers for hypoxia in their stroma, rather than in the epithelial compartment. We also showed that preserved BNIP3 function in CRC cells lowers apoptosis, and may thus be involved in alternative cell death pathways, such as autophagic cell death. Our study suggested that the biology of CRC cells can be modified by alterations in the tumor microenvironment under conditions of tumor hypoxia.

In conclusion, this thesis demonstrated the importance of CIMP in subcategorizing CRC's, which could further be characterized by TP53 mutations and microsatellite stability status. It turned out that methylation of individual genes may play a specific role in the process of tumorigenesis and tumor progression, as exemplified by the validated prognostic value of $C H F R$. Besides methylation of individual genes, hypoxia related protein expression of HIF-2 $\alpha$ and CA9 in the tumor associated stroma was associated with a poorer patient survival compared to expression of these markers in epithelial tumor cells. We have reasons to believe that promoter $\mathrm{CpG}$ island methylation and mutations of crucial cancer genes are involved in the observed more aggressive behaviour of these hypoxic tumors. Future studies are needed to unravel the pathways involved and establish the role of (epi-)genetic markers for treatment stratification of patients in addition to the traditional staging method. 
Samenvatting 


\section{Samenvatting}

Colorectaal carcinoom (CRC), ook wel kanker van de dikke darm en endeldarm genoemd, is een veelvoorkomende vorm van kanker en ongeveer de helft van patiënten met CRC sterft als gevolg van de ziekte. Op basis van het 'Tumor node Metastasis' systeem, een histologisch stadiërings systeem, worden de prognose en de behandelingskeuze van patiënten met CRC bepaald. Ondanks dat dit histologisch stadiërings systeem de overleving van groepen van patiënten accuraat voorspelt, bestaat er binnen eenzelfde stadium van CRC een variatie in het beloop van de ziekte en respons op adjuvante therapie. Dit illustreert dat er een dringende behoefte is aan nieuwe, additionele moleculaire merkers, die de respons op adjuvante therapie en de prognose van patiënten met CRC beter kunnen voorspellen. De verwachting is, dat met het verrichten van onderzoek naar de moleculaire 'pathways', die ten grondslag liggen aan deze ziekte, nieuwe moleculaire merkers ontdekt kunnen worden. Deze nieuwe, moleculaire merkers zouden, naast het huidige histologische stadiërings systeem, de prognose van patiënten beter moeten kunnen voorspellen en mogelijk nieuwe therapeutische opties voor patiënten kunnen identificeren. Het doel van dit proefschrift was het bestuderen van genetische en epigenetische afwijkingen in relatie met morfologische kenmerken en hypoxie in CRC, om met behulp hiervan, nieuwe inzichten in de biologie van CRC te verwerven en mogelijke nieuwe bruikbare prognostische merkers voor patiënten met CRC te ontdekken.

In hoofdstuk 1 wordt een introductie gegeven over de bestaande (epi)genetische afwijkingen die de ziekte CRC in drie verschillende moleculaire subgroepen verdelen: chromosomale instabiliteit (CIN), microsatelliet instabiliteit (MSI) en het CpG-eiland methylerings fenotype (CIMP). Tevens wordt er in dit hoofdstuk een overzicht gegeven van de rol van hypoxie in CRC; de cellulaire paden die hierbij betrokken zijn, en in het bijzonder de rol van het tumor geassocieerde stroma hierin.

Hoofdstuk 2 geeft een overzicht van de epidemiologie, diagnose, behandeling, prognostische en voorspellende waarde van (epi)-genetische merkers in CRC.

De eerste experimentele studie (hoofdstuk 3) beschrijft de resultaten die gevonden werden met het 'unsupervised' hiërarchisch clusteren van $160 \mathrm{CRCs}$ op basis van genetische en (epi)-genetische afwijkingen waaronder: CIMP, $\mathrm{MSI}, A P C$-, KRAS-, TP53-, en BRAF mutaties. Vervolgens werd er onderzocht in hoeverre de verkregen moleculaire clusters, correleerden met klinisch relevante patiënten gegevens, morfologische tumorkenmerken (differentiatie graad, mucineuze differentiatie, necrose, circumscripte tumorgroei, tumor 
budding en lymfocytaire infiltratie) en overleving van patiënten. Uit deze analyse bleek dat CRCs in eerste instantie op basis van CIMP onderverdeeld werden in CIMP+ en CIMP- tumoren en vervolgens op basis van TP53 mutaties verder onderverdeeld werden. Hiërarchisch clusteren was slechts in geringe mate gecorreleerd aan morfologische tumorkenmerken en patiënten overleving. Deze correlaties bleken afhankelijk te zijn van andere factoren zoals microsatelliet instabiliteit en de locatie van de tumor in de darm. Onze moleculaire cluster analyse geeft nieuwe inzichten in de onderliggende biologie van $\mathrm{CRC}$, echter de clusters bezitten geen toegevoegde prognostische waarde in vergelijking met het traditionele, histologisch gebaseerde stadiëringssysteem.

Hoofdstuk 4 richt zich op het bestuderen van de prognostische waarde van CIMP, (gedefinieerd door Weisenberger et al.) en promoter CpG-eiland methylering van 19 andere tumor suppressor- en DNA reparatie genen in patiënten met CRC. Omdat dit soort studies vaak wordt verstoord door factoren die een invloed hebben op het ziektebeloop, zoals genetische afwijkingen en chemotherapie, werd in onze studie de prognostische waarde bestudeerd onafhankelijk van $\mathrm{MSI}, B R A F, K R A S$ mutaties en tumor stadium in een populatie van patiënten met CRC die uitsluitend behandeld werden middels chirurgie. Uit deze analyse kwam naar voren dat niet CIMP, maar promotor CpG-eiland methylering van een individueel gen, het CHFR gen, een prognostisch effect toonde in stadium II, microsatelliet stabiele, BRAF wildtype $\mathrm{CRC}$; in twee onafhankelijke studie populaties van CRCs.

Hoofdstuk 5 geeft een literatuur overzicht over veranderingen die optreden in de expressie van het CHFR-gen in verschillende kanker soorten waaronder CRC en de mogelijke rol van CHFR als een biomerker.

Een belangrijke rol voor hypoxie in CRCs is gebleken uit studies die laten zien dat hypoxie gerelateerd is aan een slechtere overleving van patiënten met CRC en resistentie voor chemo- en radiotherapie. Vandaar dat we in hoofdstuk 6 de expressie bestuderen van de hypoxie gereguleerde eiwitten HIF-1 alpha, HIF-2 alpha, CA9 en GLUT1 in patiënten met CRC. Hieruit bleek dat in CRC de expressie van deze hypoxie gereguleerde eiwitten zowel in de epitheliale tumorcellen als in de tumor geassocieerde stromale cellen voorkwam. Echter, in tegenstelling tot expressie in epitheliale tumorcellen, was de expressie van hypoxie gerelateerde eiwitten HIF-2 alpha en CA9 in stromale cellen gerelateerd aan een slechtere overleving van patiënten met CRC. Dit onderzoek suggereert dat hypoxie in tumoren stromale cellen dusdanig beïnvloedt dat dit uiteindelijk resulteert in een slechtere prognose voor CRC patiënten. 
Het is bekend dat promoter CpG-eiland methylering betrokken is bij de cellulaire respons van tumoren tijdens hypoxie. Vandaar dat er in hoofdstuk 7 onderzocht is of de expressie van hypoxie eiwitten in CRC geassocieerd is met promoter $\mathrm{CpG}$-eiland methylering van genen die een rol spelen in belangrijke kanker gerelateerde processen waaronder: MGMT, CHFR, HLTF, MLH1, RASSF1A (genoom instabiliteit), p14ARF, p16INK4A (ongelimiteerde celdeling), ADAM23, TFPI2 (invasieve groei en metastasering), APC (ongevoeligheid voor groeiremmende signalen), SOCS1 (zelfvoorzienend in groeifactoren), THBS1 (onbeperkte angiogenese), en BNIP3, NEURL, RUNX3, $R A B 32$ (voorkomen van apoptose). Onze resultaten toonden aan dat niet de expressie van hypoxie eiwitten in de epitheliale tumorcellen, maar juist in de stromale cellen geassocieerd was met promoter CpG-eiland methylering van diverse genen die een belangrijke rol spelen in kanker gerelateerde processen. Dit onderzoek suggereert dat genmethylering in hypoxische tumoren niet een willekeurig proces betreft, maar juist gekenmerkt wordt door methylering van specifieke kanker gerelateerde genen, waardoor deze hypoxische tumoren in staat zijn zich agressiever te gedragen.

Ten slotte is in hoofdstuk 8 onderzocht of tumorcel dood en hieraan gerelateerde (epi-)genetische afwijkingen in de epitheliale tumorcellen, geassocieerd zijn met de geobserveerde slechtere overleving van CRC patiënten met expressie van hypoxie eiwitten in stromale cellen. Hiervoor werd er in epitheliale tumorcellen gekeken naar afwijkingen in apoptose regulerende genen zoals TP53 en BNIP3, en onderzocht of deze gen afwijkingen gecorreleerd waren met de slechtere overleving van CRC patiënten met stromale expressie van hypoxie eiwitten HIF-2 $\alpha$ en CA9. Uit dit onderzoek bleek dat de geobserveerde slechtere prognose van CRC patiënten met expressie van HIF-2 $\alpha$ in stromale cellen, gecorreleerd was met wild-type TP53 en onafhankelijk van de methylerings status van BNIP3. Kennelijk draagt ongemuteerd TP53 ertoe bij, dat tumoren met stromale expressie van hypoxie eiwitten progressie laten zien, in tegenstelling tot tumoren met expressie van hypoxie eiwitten in de epitheliale tumorcellen. Verder bleek dat een behouden functie van het BNIP3 gen resulteerde in minder apoptose, en dus mogelijk betrokken is bij een alternatief tumorcel dood mechanisme, zoals 'autofagie' gereguleerde tumorcel dood. Onze studie laat zien dat de biologie van CRC cellen gemodificeerd wordt door hypoxie in het tumor geassocieerde micromilieu.

Concluderend leveren de resultaten van dit proefschrift nieuwe inzichten in de ontstaanswijze van colorectale tumoren. Men kan op basis van (epi-)genetische afwijkingen zoals CIMP, TP53 mutaties en microsatelliet 
instabiliteit, CRCs onderverdelen in aparte subgroepen, waarbij individuele epigenetische merkers zoals CHFR methylering, een onafhankelijke en gevalideerde prognostische merker bleken te zijn. Naast DNA promotor methylering van individuele genen, was de expressie van hypoxie eiwitten HIF$2 \alpha$ en CA9 in stromale cellen gerelateerd aan een slechtere overleving van CRC patiënten en aan methylering van specifieke kanker gerelateerde genen. Toekomstige studies zullen de betrokken celbiologische processen moeten ophelderen en het potentiële belang van epigenetische merkers voor het bepalen van de therapie in CRC patiënten moeten aantonen. 
Dankwoord 


\section{Dankwoord}

Het dankwoord is het meest frequent gelezen hoofdstuk van een proefschrift.

Gek genoeg bedankt de gemiddelde promovendus in dit dankwoord zijn vrienden, opa, oma en de buren, en nog net niet zijn cavia of diepzeeschildpad. Waarvoor? Hetzelfde principe dat je op je eigen verjaardagsfeest rond rent met bitterballen, mosterd en lege flesjes bier voor vrienden, opa, oma en de buren! Daarbij komt dat deze vrienden, opa, oma en de buren, je voor gek verklaren tijdens je promotie en de wederkerende vraag stellen: "Wanneer is het nu eens af?" of deze: "Waarom maak je het niet gewoon af?" of deze: "Loopt alles volgens de planning?"

Dat is een goede in wetenschapsland waar het principe 'Tomorrow never dies!' zegeviert. Tijdens een promotietraject zit je in een spannende, eeuwig durende treinreis op je werk, na je werk, op vakanties, ga zo maar door. Als je wilt, kun je ook aan je onderzoek werken tijdens $30 \mathrm{~min}$. fietsen in de fitnessclub. Terwijl andere zwetende medeburgers tijdschriften lezen zoals: Privé, Linda en de Telegraaf, lukte het mij om gemiddeld 1 artikel uit: JAMA, JNCI, CCR of JCO te lezen. Uiteindelijk na al het gefitness zegt de conducteur: "Meneer Cleven, $u$ heeft uw bestemming bereikt." Je stapt na alle hoogtepunten en ontberingen uit te trein waarin je jaren hebt gezeten; een tijd waarin relaties werden gewisseld, baby's geboren, banen gewisseld, er vielen zelfs doden! Ik heb nog net geen oorlog meegemaakt. Plotseling sta je dus op het pad in de Minderbroedersberg 4-6, en dat doet me denken aan een scène uit 'Monty Python and the Holy Grail'. Een ridder ligt op een eenzaam zandpad met afgehakte onderste en bovenste extremiteiten en schreeuwt vervolgens tegen zijn opponent: "Come on, tis but a scratch- A scratch? Your arm's off.- l've had worse, come here l'll bite your legs off!"

Na deze korte introductie m.b.t. het promotietraject, moet ik toegeven dat onze volksheld Lee Towers gelijk heeft met zijn lied vanaf de Rotterdamse haven 'You never walk alone!'.

Vol enthousiasme en een hoop wetenschappelijke bagage, dacht ik, startte ik aan een nieuw onderzoeksproject met als doel uiteindelijk te promoveren. Ik klopte aan bij mijn promotor prof. dr. Adriaan de Bruïne.

Beste Adriaan, hoe kan ik nu in godsnaam formuleren wat jouw rol is geweest in het tot stand komen van dit proefschrift? Aangezien god niet bestaat, zal ik proberen het chronologisch in verhaalvorm uit te leggen.

$\mathrm{Na}$ ons eerste gesprek op de afdeling, nam je mij op sleeptouw in de wetenschappelijke wereld van de afdeling pathologie te Maastricht. Bij het eerste onderzoeksvoorstel dat ik schreef, kan ik mij nog herinneren dat je zei: "The sky is the limit meneer Cleven". 
$\mathrm{Na}$ een jaar in vitro werk met cellijnen onder hypoxische omstandigheden, zonder bruikbare resultaten, bleek er inderdaad een 'sky' te zijn. Jij maakte snel de beslissing om het onderzoek te concentreren op in vivo werk in een serie van dikke darm tumoren dat uiteindelijk geresulteerd heeft in het huidige proefschrift. Tijdens deze lange periode nam je mij mee naar verschillende congressen waarbij ik nieuwe onderzoeksdata mocht presenteren. Naast het leerzame aspect hiervan, was het altijd ontzettend gezellig: je regelde leuke hotels, heerlijke etentjes en minstens een 'City-tour' per vreemde stad. Deze gezelligheid resulteerde in de beruchte: 'The flying burito with a lot of sour cream' op het nieuwe driedelig pak van een perifere patholoog in Brighton; tot de bratwurst met curry om zes uur 's ochtends in hartje Berlijn. We waren om vier uur vertrokken vanuit Maastricht en eenmaal aangekomen in Berlijn hadden we geen muntgeld voor een treinkaartje. Het kopen van een bratwurst resulteerde in muntgeld, maar je was volgens mij blij en ik overigens ook, nadat de bratwurst met curry om zes uur in de ochtend in de prullenbak belandde.

Naast het leren om belangrijke details te signaleren uit een wereld van resultaten, heb ik door jou geleerd snel te lezen, met behulp van het boek 'gebruik je hersenen'. Dit boek lag op een ochtend op de bijrijderstoel in onze carpool sessie naar Venlo. Een stille hint!

Af en toe was ik ongeduldig, ik vuurde dan simpelweg een kruisraket met kernkop af. Bij de aanblik van dit projectiel aan de horizon, brommelde jij je tot een kolonel in het kaliber van 'Apocalypse now' William Kilgore: "Charlie don't surf, but I love the smell of napalm in the morning". Het resultaat was, dat de kernkop terugkwam op mijn eigen grondgebied en vervolgens ontplofte. Dat was verhelderend, aangezien ik na zo'n Hiroshima momentje, weer door de weinige bomen het bos kon zien!

Adriaan, ik denk dat ik mijn achternaam 'Cleven' waar heb gemaakt!

Jij bent de grondlegger van dit boekje en ik wil je bedanken, niet alleen voor de goede en langdurige begeleiding, maar ook voor de inhoudelijke vrijheid die je mij gegeven hebt en de ontzettend gezellige tijd die hiermee gepaard ging.

Voor mijn promotietraject had ik mijn hobo al lang ingewisseld voor een stevige elektrische gitaar met versterker. Dit heeft mijn promotor prof. dr. Manon van Engeland vaak moeten ervaren.

Beste Manon, laat me beginnen te zeggen dat het niet raar is dat wij elkaar nog ontmoet hebben in de prachtige wereld van de wetenschap. Jaren geleden mocht ik, als broertje samen met mijn vader mijn zus en haar discovriendinnetje 's avonds met de auto ophalen. Jij bevond je ook onder deze discovriendinnetjes. Op de terugweg naar city Susteren zagen alle inzittenden rondom middernacht een schotelvormig object met rode lampen in een verlaten Susters weiland. We dachten allemaal dan nu eindelijk een UFO gespot te hebben in de achtertuin van de Hommele Hei. Slechts één 
discovriendinnetje trok deze hypothese in twijfel en ging op onderzoek uit. Bij thuiskomst volgde er een telefoontje: "Ja, hier met Manon. Neen, het is geen UFO maar een bietenwagen van een boer die daar zo stond te blinken." Jaren later kwam ik je weer tegen in het onderzoekslaboratorium waar jij leiding geeft. Ideeën en plannen waren er genoeg, maar wat zonder onderzoeksgeld? Manon, jij hebt meerdere bietenwagens vol met euro's in mijn onderzoek gepompt. De reagentia, een analist, high throughput sequencing; zonder jouw investering had er geen letter geschreven kunnen worden. Je hebt me alle do's en dont's uitgelegd in de wereld van de epigenetica, het stappenplan bij het schrijven van een helder hoofdstuk en als vrouw heb je mij ook weten te overtuigen dat een lay-out essentieel is. Terugkomend op de gitaar, Jimi Hendrix introduceerde zichzelf tijdens zijn eerste concert in Europa met het doormidden breken van zijn gitaar en het vervolgens in brand steken van zijn schitterend solo-instrument. Dit soort sessies hield ik af en toe ook in jouw Headquarter. Je keek ernaar, je luisterde ernaar en met een symbolische emmer koud water bluste je het vuurtje, waardoor ik onbeschadigd verder kon gaan met mijn wetenschappelijke carrière. Manon, bedankt voor alles.

Een lotgenoot! Net zo goed als moeder Theresa de lotgenoot is van Gandhi, had ik tijdens mijn promotietraject ook een lotgenoot.

Beste dr. Sarah Derks, mijn co-promotor. Sarah, wat ik bedoel met lotgenoot is dat het prettig was te weten dat ik niet de enige idioot was die op een vrije dag een agarose gel stond op te dweilen. Dit nadat deze omslachtig voorbereide gel had besloten na de eerste sequence run, de polonaise te nemen tussen de glasplaten door tot op de grond; terwijl ander vrije collega's verkleed de polonaise liepen op het Vrijthof. Beiden waren we zeer kritisch naar elkaar toe, wat vaak verhitte discussies opleverden die meestal eindigden met: "Ja, ik begrijp wat je bedoelt, maar ik heb gelijk!" Daarnaast konden we als buren samen goed dineren, met Joep en mij als wijn- en whiskydeskundigen: prachtige tijden!

Laat me eindigen te zeggen dat er meer artsen zouden moeten zijn zoals jij, die hun wetenschappelijke ideeën zo plannen, dat ze een klinisch toepasbare relevantie hebben. Ik ga ervan uit dat ik op de hoogte blijf van je carrière en eventueel een Tweede Kamerdebat met je voer! Sarah, bedankt voor de hulp en het fantastische stuk over CHFR.

Het lab: Ingrid, bedankt voor het uitleggen van de IHC en cellijn basisprincipes, jij hebt mij mijn eerste labboek gegeven, dat ik kon vullen met allerlei experimenten. De Mark en de Mork, bedankt Margriet en Leentje voor het openstellen van jullie IHC faciliteiten. De eerste TP53 en APC mutatieanalyses, ga zo maar door, een DNA vrije kamer, soep en de ingrediënten. Bedankt Guido, Andrea en Angela. Rik, de verloren zoon, 
bedankt voor de HIF-1-alpha oplossing, de pH-waarde van de wasbuffer was zeer essentieel! Peter en Joep, bedankt voor jullie mannelijkheid!

Charlies Angels: Sandra, Kim en Kathleen, mega bedankt voor al die primer sequencies, epjes vol met reagentia, pipetpunten en pipetten. Onderzoek is onmogelijk zonder jullie voortdurende back-up!

Beste Angela, je hebt veel werk uit mijn handen weggenomen en je hebt me uitgelegd wat een logische sample codering is. Ik heb je uitgelegd waar "Der, Die, Das Party-zone is in Maastricht", en welke mannelijke tube "Der, Der, Der Falsche ist!"

Andere lotgenoten: Veerle, Ingrid, Iris, Femke, Coen, Kim (Survival of the Fittest) en Debbie. We zijn allemaal goed terecht gekomen, maar Debbie de meeste dromen zijn bedrog!

$\mathrm{Nu}$ aangekomen bij een ander lotgenoot, paranimf en patho-collega: Marjolein Lentjes-Beer. Beste Marjolein, wat heb ik vaak met jou kunnen lachen op de wetenschapskamer. We hadden op vrijdagmiddag ons verdiende you-tube halfuurtje: "Hi my name is Veronique, but recently I changed my name in REFLEX, REFLEX, I like to put the tip of my tong into your earhole, you sick b....", Gunter. En nu op zijn Duits!

Over patho-land gesproken, daar zal ik zeer zeker een aantal zwaargewichten van moeten bedanken. Collega arts, $u$ bent gewaarschuwd: zodra er tegen $u$ gezegd wordt dat uw proefschrift klaar moet zijn voordat de opleiding begint, is het al te laat! Vergelijk het met het medisch anticipatie krediet van de ABNAMRO na het behalen van uw propedeusediploma!

Het geweldige team uit Venlo, ik kon bij jullie een half jaar van mijn perifere stage lopen. Naast het leren van het vak op zijn pragmatisch en degelijk perifeers, gaven jullie meteen aan dat er tijd was voor onderzoek. De dieselmotor van Venlo, ook wel Siep Wouda genaamd, hield in zijn eentje de fabriek in Venlo draaiende terwijl Adriaan en ik in een afgesloten kamer zonder telefoontjes van de kliniek, werkten aan een wetenschappelijk hoogtepunt. Jij beste Siep, kwam om half vijf met je jas aan polsen hoe het was in wetenschapsland, 80 coupes lichter en 2 obducties rijker verliet je tevreden het pand! Bedankt voor de tijd die jij letterlijk voor mij en Adriaan creëerde. Dat geldt tevens voor het andere teamlid uit Venlo, Ariënne van Marion. Ariënne, bedankt voor de uitleg over het beenmerg, de kleine lymfkliercelletjes en de fles Champagne. Tja, ik zal hem zelf moeten betalen, hopelijk kom je naar de Domkeller in Aken, dan toosten we samen!

Het kloppende team uit Heerlen, een heel jaar lang onder de hoede van jullie deskundigheid! Jullie hebben mij onder de loep genomen en flink bijgeschaafd wat mijn vorming tot patholoog heeft bespoedigd. Ongelooflijk wat ik bij jullie allemaal heb kunnen leren! Het stellen van de diagnose folliculair lymfoom 
zonder immuno's (Paul Theunissen), "een microscoop is ouderwets", "dicteer wat je ziet en concludeer ondubbelzinnig" (Marius Nap). Ik begrijp alle afkortingen in de gynaecologiewereld: CIN, VIN, VAIN, EIN, CGIN (Nathalie Vandevijver). En ja, ik weet dat Bier belangrijk is, dat de kassa rinkelt na een send rapport en wat het verschil is tussen boven en onder, voor en achter, als je op de aardbol van de prostaat staat (Ruud Clarijs). Jullie alle vier, enorm bedankt voor de goede opleiding in de baas zijn tijd, wat dus inhield dat ik mijn boekje in de weekenden en avonduren eindelijk eens kon gaan afschrijven!

Het grootste team uit Maastricht: de assistenten (van Mitch, Lance, Branda tot mister en misses Lover Lover) en stafleden, ik bedank jullie, als jullie samen met mij feesten op 1 juni in de Domkeller! Laten we er een feestje van bouwen, dan kunnen we op maandag weer soepel de weefsels snijden met een mes zonder mes!

De stafdames en assistenten van divisie drie, vier en nog meer? Bedankt voor the Holiday!

Het management: Cor, Elly, Audrey en Tiny Wouters, bedankt voor het werk achter de schermen. Het hele secretariaat, bedankt!

De opleider: dr. Ann Driessen, beste Ann, bedankt voor de soepele planning van de wetenschap in mijn eerste jaar en de soepele planning rondom 1 juni!

Prof. dr. Fré Bosman, U heeft nagenoeg mijn hele proefschrift gereviewed en voorzien van praktische tips, enorm bedankt voor dit alles!

Prof. dr. Mat Daemen, ik kreeg een 'go' op mijn 'no go-gesprek' en de ware, dankbare tip: "Blijf realistisch in je doelstellingen en behoud in alles wat je doet plezier!"

Talking about 'Pulp Fiction', It's been a while prof. dr. Brad Wouters, many thanks for our productive collaboration! 
Thanks for editing this chapter mister Granda: "In love there are no buts!"

Het 'bystander-effect', ja, schwessie Anouk, daar weet jij alles van als afgestudeerd psychologe werkzaam in Brazilië samen met je lieve man Markie Mark! Geweldig dat we deze dag samen gaan vieren.

Lieve Mutti, ik weet dat je trots bent op mij, ik ben ook trots op jou en laten we genieten van alle goede dingen die het leven ons te bieden heeft. Bedankt voor de spellingscontrole en de op lange termijn zo belangrijke, elementaire sturing die je mij in mijn leven gaf als onwetende, middelbare scholier puber!

Mijn spudselhaze, lieve, lieve Annette. Bij onze eerste treffen in Berlijn, verkondigde ik nonchalant dat ik zo goed als klaar was met promoveren. Dat was in 2008! Jij bent de enige die mij nooit gevraagd heeft wanneer het nu eens af was. Je regelde een super appartement in Lissabon, waar ik niet alleen kon werken, maar waar we tegelijkertijd konden genieten van een vakantie. Je faciliteerde vaak genoeg een continue werkomgeving in het vliegtuig, uit het vliegtuig, in de trein, uit de trein, in de stationhal, uit de stationhal; en dit met behulp van meerdere laptops, USB-sticks en een hoop koffie en lekker eten. $\mathrm{Ja}$, ook ik verheug me enorm op de komende vakantie, welteverstaan zonder $\mathrm{PhD}$ laptop! Tenslotte, Bono zingt voor jou: "A woman needs a man like a fish needs a bicycle, but a man needs a woman!"

Arjen Cleven

Aachen, maart 2012 
Curriculum vitae 
192 


\section{Curriculum vitae}

Arjen Cleven was born in Rinteln in Germany on January $13^{\text {th }}$, 1977. After completing Athenaeum at the Serviam Lyceum in Sittard in 1997, he started studying Biological Health Sciences (1997) and Medicine (2000). He performed research for his master thesis at the department of General Practise, Epidemiology and Statistics at Maastricht University under supervision of Dr. H.E.J.H. Stoffers. In 2002 he received his master degree in Biological Health Sciences on the topic: Prediction of the outcome of peripheral arterial disease using the ankle-brachial pressure index. After his graduation in 2002 he started as a research assistant in the cancer pathology laboratory of Prof. dr. M. van Engeland and Prof. dr. A.P. de Bruïne at the department of Pathology of Maastricht University Medical Center. In 2003 he continued his research and started his Ph.D. program by studying the prognostic implications of hypoxia and promoter $\mathrm{CpG}$ island methylation in colorectal cancer, which resulted in this dissertation.

In 2006 he completed Medical School and in 2008 he started his residency training Pathology at the Maastricht University Medical Center, Maastricht. 
List of publications 


\section{Full papers}

Cleven AHG, Kester AD, Hooi JD, Knottnerus JA, van den Brandt PA, Stoffers HE. Cardiovascular outcome stratification using the ankle-brachial pressure index. European Journal of General Practioners, 2005 Sep-Dec; 11(3-4):10712.

Cleven AHG, van Engeland M, Wouters BG, de Bruïne AP. Stromal expression of hypoxia regulated proteins is an adverse prognostic factor in colorectal carcinomas. Cellular Oncology, 2007; 29:229-240.

Cleven AHG, Wouters BG, Schutte B, Spiertz AJ, van Engeland M, de Bruïne AP. Poorer outcome in stromal HIF-2 $\alpha$ and CA9 positive colorectal adenocarcinomas is associated with Wild-type TP53 but not with BNIP3 promoter hypermethylation or apoptosis. British Journal of Cancer, 2008; 99:727-33.

Smits KM, Cleven AHG, Weijenberg MP, Hughes LA, Herman JG, de Bruïne $A P$, van Engeland M. Pharmacoepigenomics in colorectal cancer: a step forward in predicting prognosis and treatment response. Pharmacogenomics, 2008; 9(12): 1903-1916.

Dubois L, Magagnin MG, Cleven AHG, Weppler SA, Grenacher B, Landuyt W, Lieuwes N, Lambin P, Gorr TA, Koritzinsky M, Wouters BG. Inhibition of 4EBP1 sensitizes U87 glioblastoma xenograft tumors to irradiation by decreasing hypoxia tolerance. International Journal of Radiation Oncology Biology Physics, 2009; 73(4):1219-27.

Cleven AHG, Derks S, Smits KM, Weijenberg MP, Herman JG, de Bruïne AP and van Engeland M. CHFR promoter $\mathrm{CpG}$ island methylation is an indicator of poor prognosis in stage II microsatellite stable colorectal cancer. Submitted for publication.

Cleven AHG, Derks S, Smits KM, van Engeland $M$ and de Bruïne AP. Computer assisted (epi-)genotypic categorization of colorectal adenocarcinoma identifies CIMP and TP53 mutational status as leading classification tools. Submitted for publication. 
. 

. 